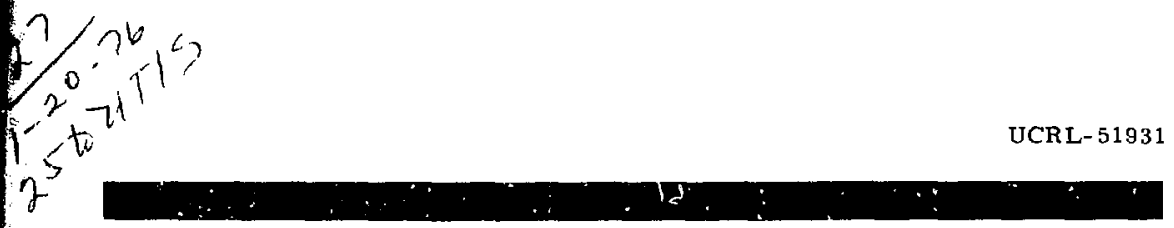

\title{
A NUMERICAL INVESTIGATION OF THE PULSED NF3 + H2 CHEMICAL LASER USING A MODEL WHICH INCLUDES ROTATIONAL RELAXATION AND SEMI-CLASSICAL LASER EQUATIONS.
}

John R. Creighton

(Pn. D. Thesis)

September 1, 1975

Prepared for U.S. Energy Research \& Deyelopment Administration under contract No. W-7405-Eng-4B

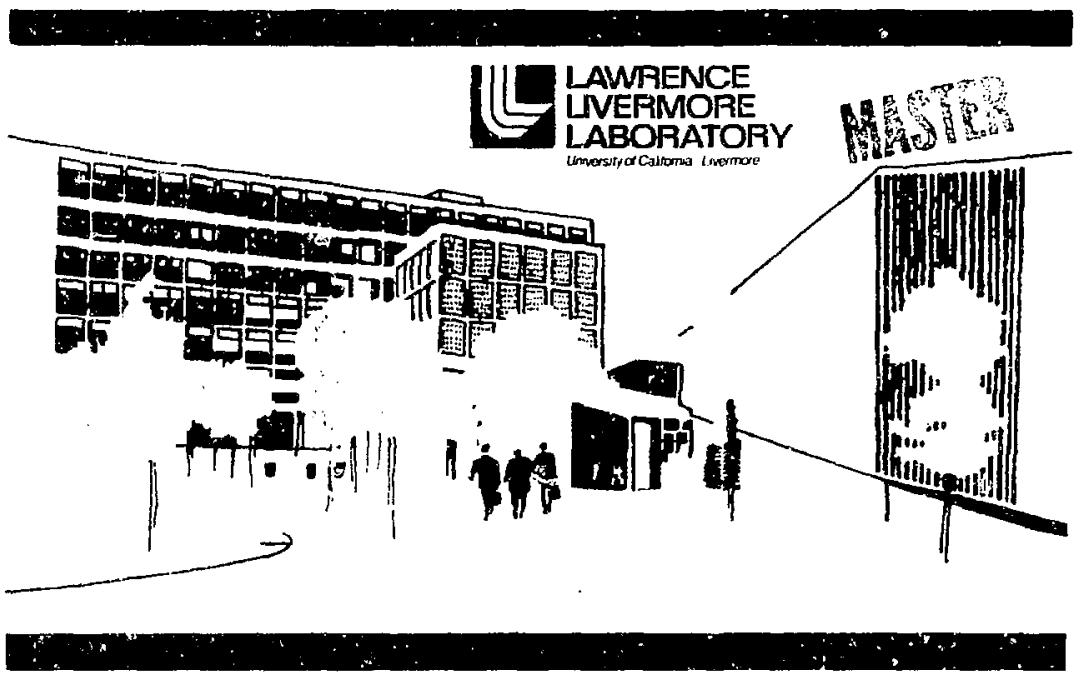




\section{NOTICE}

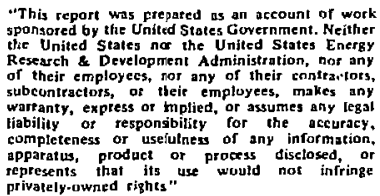

privately-owned rights"

Printed in the United States of America Available from

National Technical Information Service

U.S. Department of Commerce 5285 Port Royal Road

Springfield, Virginia 22151

Price: Printed Copy \$ $\$$

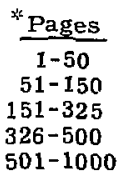

NTIS

Selling Price

$\$ 4.00$

$\$ 5.45$

$\$ 7.60$

$\$ 10.60$

$\$ 13.60$ 


\section{近 \\ LAWRENCE LNEPMORE LABORATOPY

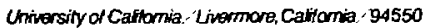

UCRL- 51935

\section{A NUMERICAL INVESTIGATION OF THE PULSED NF3 + H2 CHEMICAL LASER USING A MODEL WHICH INCLUDES ROTATIONAL RELAXATION AND SEMI-CLASSICAL LASER EQUATIONS \\ John R. Creighton \\ (Ph. D. Thesis)}

Septemlier 1, 1975

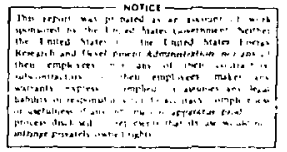


John $R$. Creighton

1 Nov, 1975

Engineering - Applied Scimle

A NUMERICAL INVESTIGATION OF THE

PULSED $\mathrm{NF}_{3}+\mathrm{H}_{2}$ CHEMICAL LASER

USING A MODEL WHICH INCLUUES

ROTATIONAL RELAXATION AN!

SEMI-CLASSICAL LASER EQUAT'TONS

ABSTRACT

haveforms and population distributions have been calculated by a numerical model and compared with experiment for an electric-discharge-initiated, pulsed $\mathrm{NF}_{3}+\mathrm{H}_{2}$ chemical laser. The model treats each vibrational-rotational state separately, allowing rotational relaxation between adjacent states as well as vibrational relaxation and lasing arcording, to P-branch selection rules. Simplified semi-classical laser equations, roughly equivalent to laser rate equations, are used; and waveforms for each P-branch transition arr obtained. Simultanfous solution of a simplitied set of chemical kintetic rate equations gives the pumping rate for each statf.

Calculated waverorms agree with experiment and show several features not seen when rotational equilibrium is assumed: simultaneous lasing on many transitions, ciscade behavior, spikes due to laser relaxation oscillation;, 
non-Boltzmann rotational distributions, and "hole burning" in the population distributions. The calculations give insight into the physical phenomena governing, the shape and duration of the wavelotins. The effect of varying certain parameters, relaxation rates, temperature, pressure, and diluents, is studjed. Bost fit to experimental waveforms is obtained when the rotational relaxation rate and collisional line broadening rate are approximately equal at about 10 times the hard sphere collision rate.

The IXION romputer code, developed for these calculations, is described in detail. In addition, an analytic model is presented which accounts for major features of the total (all transitions) output waveform of the laser assuming rotational equilibrium, a steady state laser model, and constant temperature. A second computer code. MINOTAR, was developed as a general purpose chemical kinetics code. It verifies the analytic model and extends the results to adiabatic reactions where the temperature varies, and san yield waveforms using the assumptions of rotational equilibrium and a steady state laser. The MINOTAR code, being general, can also be used for chemical kinetics problems such as air pollution and combustion. 
(ivingen

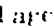

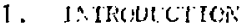

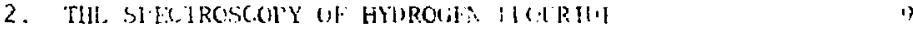

2.1 Incrodurt ion

2.2 Vibrational-Rotational smect runi is

2.3 sonret iur Ritiles 1?

3. FXIFRTMETAL BACRLRGOND 1:

3.1 Apparatus I's

3.2 Waveforins 16

3.3 1) jssociation of $\mathrm{NF}_{3}$ by
the Electric bischargr.

the Electric bi schargr
Tabie 3.1

Figures $3.1-3.13$

4. Ai. MALYTTC NOUEL CF THE CHEMICAI IASIR

4.1 the Steady-State laser Mote. 36

4.2 Chemical Pumping of the HIt I aser 40

4.3 The Fffects of Vibrativial

4.4 Variation ol laser Fulse friergy with firessure 48 Figures 4.1 - 4.3 Th

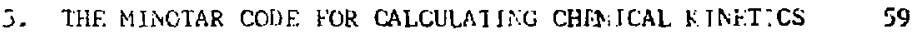

5.1 Introduction 59

5.2 The Gear Method for

Solving Uifferential Equations 60

5.3 tetails of the Code

5.4 Calculations of $\mathrm{HF}$ Kinetics $7 i$

Tables $5.1-5.3 \quad 77$

Fi gures $5.1-5.8 \quad 80$

6. LASER EQUATJONS FOR A MORE DETAILEU NOULI. 85

6.1 Introduction 85

6.2 Werivation of Semi-Classical Laser Equations for a Thre:-level Cascarle 88

6.3 Steady-State Values 101

i.4 Stability of Two Level Laser Equations 108

Figures $6.1-6.6$

122 


\section{CC?IESTS}

rage

7. THE IXIGN CORIPUTER COIJE 128

7.1 lintroduction 128

7.2 Crdering of the Nolecular States 131

7.3 The romulation: Equation 132

7.4 The Eolarization Equation 144

7.5 The Electric Field Equation 149

7.6 Simulation of Vetector Response 152

7.7 Temperature Rise 154

7.8 Codes to Rearrange cutmut 156

Tables $7.1-7.2$ 158

Figures $7.1-7.5 \quad 160$

8. CALCLLATLi) WIVHEORMS A:H LETAILS (F LASER
OPERATIOA

8.1 Introduction 165

8.2 The iominal Calculated haveforms 165

8.3 Sequence of Events vuring the Laser Pulse 173

8.4 How Valid is the Assumptiun of Rotational

8.5 Comparisun of the IXION Code Results with
Earlier Coles

Figures $8.1-8.25 \quad 192$

9. VARIATIIN OF TARARIETLRS 210

9.1 Variation of Rotational Relaxation Rate 210

9.2 Addition of Diluent 214

9.3 Calculation of Approximate Rotational

Equilibrium 216

9.4 Mlodification of the Rotational Relaxation Rate as a Function of Rotational State

$\begin{array}{ll}9.5 \text { Vibrational Relaxation Rati } & 219\end{array}$

9.6 Fraction of $\mathrm{NF}_{3}$ Uissociated 22 ,

9.7 Variation of Laser Cutput with Temperature 221

9.8 Total Output Eneray as a Funetion of Total 223

$\begin{array}{ll}\text { Pigures } 9.1-0.20 & 223\end{array}$

10. CONCLIISION 246

ACKNOWLELGENENTS $\quad 250$

RLFERENCES 
Coxfer.ts

$\operatorname{lin} \theta$

Appeidix A - Vibrationa1-Rotal ional Snectrum of Hydropen Finilidn

liablas AI - A3

26.

26.3

Appendix $B$ - Thr Role of inturetical stability

in the Solution of Chemical Chaj $f^{-}$Reactions

Apjendix C - Conmutacors for lif: Hamiltonian of tquation 6.1

Appendix $D-$ A Simple Mnalvic Soutel for

Rutational Relaxation

Table $)$

ficures $11-42$

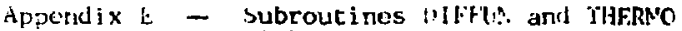

irom the MJNOTAR Computer Cove

Appendix $F$ - Subruutines ilFFtXP ar. CHEMIST from the Iison code 
1. TNTROLUCTION

This raport preserts ghysical models which explain the major feirures of the pulsed $\mathrm{NF}_{3}+\mathrm{H}_{2}$ hydrogen flouride laser initiated by a short duration electric discharge. The work was performed over a period of several years in support of the experimental program of Pearson, Gregg, and co-workers (1973a, 1973b). The pulsed laser was chosen in an attempt to obtain short duration, high intensity pulses for laser fusion. In the $\mathrm{NF}_{3}+\mathrm{H}_{2}$ laser the electric discharge dissociates a fraction of the $\mathrm{NF}_{3}$ to give $\mathrm{F}$ atoms and other species which are not important to laser action. The chemical reaction

$$
\mathrm{F}+\mathrm{H}_{2} \stackrel{\mathrm{H}_{1}}{ } \cdot \mathrm{HF}+\mathrm{H}
$$

creates vibrationally and rotationally excited HF which lases. It is also possible to buizd a pulsed ht laser using a mixture of $F_{2}$ and $H_{2}$. so that the chemical reaction

$$
\mathrm{H}+\mathrm{F}_{2} \stackrel{\mathrm{k}_{2}}{\longrightarrow} \mathrm{HF}+\mathrm{F}
$$

produces additional excited $\mathrm{HF}$, and alsc creaces a new $\mathrm{F}$ atom to replace the one consumed in reaction 1.1 , constituting a chain reaction. This report is primarjly concerned with the $\mathrm{NF}_{3}+\mathrm{H}_{2}$ laser which depends only on reaction 1.1 ; but the analytic theory is extended to cover the chain reaction which includes 1.2. This report doesn't explicitly consider the "continuous wave" HF laser where the chemical reactions take place in flowing mixtures of reactant gases yielding HF, although the chemical kinetjcs is similar. 
When this work wiss brepun in 1970 a tumber of experiments had been reported in the 1 iterature and sums modeling work was in progresis. Bibliographies of published HF laser work up to mid-1971 are given in Chester and Hess (1972) and Dobrati (1972). My initial numerical calculations showed that with a couple of assumptions an analytic nodel could be constructed for the $\mathrm{NF}_{3}+\mathrm{H}_{2}$ chemical laser. The reaction of $\mathrm{H}$ with $\mathrm{NF}_{3}$ is orders of magnitude slower than reaction 1.1 , so the laser is controlled by reaction 1.1 . A fast electric discharge dissociates $\mathrm{NF}_{3}$ to give an initial concentration of $F$ atoms, which are consumed by reaction 1.1 . It can be shown that laser output is generally proportional to the rate of Eormation of HF, which can be calculated from Eq. 1.1. This lead, to a non-linear rate equation which would require a minerical solution except that we can make a further assumption. Because the initial concentration of $F$ atoms is small we can assume that the concentration of $\mathbf{H}_{2}$ is constant and get a solution for the laser pulse shape, which is an exponential decay at a rate governed by the consumption of $F$ atoms. This is discussed in Section 4 añi is extended to include reaction 1.2 as well as vibrational deactivation and recombination. This simple theory agreed with experiment when the measured rate constant for reaction 1.1 was used. This analysis led to a new method of determining chemical kinetic rate constants (Creighton, 1972 and Pearson. et al.. 1972b). A number of experiments had been done using $\mathrm{NF}_{3}$ with a variety of hydrocarbons. Kinetic rate constants 
were obtained by measuring the decay constants of the waveforms (Crejghton, 1972). Eight cr these kinetic rates agreed with experimental rates tabulated by Trotman-Dickenson (1967). The rates for the other eight hydrocarbons were apparent ly th: first reported. While this work was in progress Pummer and Kompa (1972) published a similar analysis and experimencal rates for a few reactions. A year later Igoshin and co-workers (1973) described a measurement of the kinetic rate for the reaction

$$
\mathrm{F}+\mathrm{U}_{2} \longrightarrow \mathrm{DF}+\mathrm{D}
$$

using a similar technique. Apparently unaware of the earliei work they developed cheir tecinique independent $1 y$ and erroneously claimed to have made the first direct measurement. of this rate. However, they made a significant contribution by refining the technique to get quite accurat: results. Numerical solutions of the chemical kinetics equations are required if the temperature is allowed tc vary because the equations become highly non-linear due to the temperature dependence of the rates. A computer is also required to treat tho kinetics in more detail because of the large number of variables. For exampie if each vibrational state of $\mathrm{HF}$ is treated as a separate species and recombination and vibrational deactivation reactions are included the complete kinetics involves about 15 species and 50 to 100 reactions.

Numerical solutions of the kinetics is not straighttorward, as it might appeir. The equations are Jrdinary 
differential equations but they are "stiff" in the sensir that they involve time constants that differ by many orders of magnitude. Some of the time constants are in the sub-nanosecond range and others in the mi.crosecond range. standard numerica1 techniques, such as Runge-Kutta require that the time step of the numerjcal method be less than the shortest time constant, to maintain numerically stable solutions. Thj.s uses excessive amounts of computer time, and leads to disastrous round-off and truncation errors.

Emanuel (1964) has gotten around this problem by taking advantage of the fact that stealy state approximations can be invoked throughout most of the problem. This eliminates the short time constants at the cost of using sophisticated logic to test the validity of the steady state approximation. For the present work the MINOTAR* code was constructed using a nunerica1 method devised by $C$. W. Gear (1971). This method gives stable solutions when the time step is orders of magnitude larger than the shortest time constant, provided a1l variables are changing slowly. (Gear's method is discussed in Appendix B.) The superiority of this method is illustrated by an example. The MINOTAR code was able to calculate and $\mathrm{H}_{2}+\mathrm{F}_{2}$ explosion in 300 time steps, using less than 10 seconds of CDC 7600 computer time. The same calculation using Emanuel's (1971) NEST code was not complete after using 10 minutes on the CDC 7600 computer (Rapagnani, 1973).

"The proper spelling is MINOTAUR but the computer accepts only seven-letter names. 
Kinetics calculations using the MINOTAR code are presented in Section 5 and compared with the analytic model.

The analytic and the kinetics codes can calculate the overall laser waveform. If rotational equilibrium within each vibrational level is assumed, the codes can also calculate population inversions and small signal gains. They cannot calculate laser saturation effects. Turner, Adams and Enanuel (1973) have added a steady-state laser model to the NEST code. This model treats laser saturation, but because it assumes rotational equilibrium it calculates lasing on only one $P$-branch transicon at a time for any pair of adjacent vibrational levels. As the temperature aid populations change other transitions lase, but only one at a time. This is known as J-shifting (Chester, 1970; Emanuel, 1971). Experiments with pulsed HF lasers show simulcaneous lasing on several transitions between pairs of vibrational levels. To calculate this a molel must include rotational nonequilibrium.

The IXION computer code was constructed to study the effects of rotational non-equilibrium in the HF chemical laser. The population of each vibrational-Iotational state is treated separately. Each state receives an appropriate fraction of the HF which is rormed by the chemical reaction. Rotational relaxation to ndjacent states is allowed. Vibrational relaxation and lasinf follow p-branch selection rules. Lasing, is treated by means of semi-classical laser equations. These are derived in Section 6 . By using a 
steady-state approximation on a phase angle the semiclassical equations are simplified, but still bive more accurate time constants than the usual laser rate equations. Both semi-classical and rate equations give a much more accurate waveform than a steady-state laser model. Section 6 also shows that the dipole selection rules prevent nonresonant interactions between two laser transitions in a cascade. The only coupling is due to sharing the population of a common state. Relaxation oscillations are a significant part of the experimental laser waveform, so there is an extensive study of the stability of laser equations in Section 6. Instability shows up as a sequence of spikes. Stable solutions are equivalent to steady-state solutions and have smooth waveforms which follow the pumping rate after an initial transient. Calculated waveforms show examples of both stable and unstable waveforms. Section 7 gives a detailed descrintion of the IXION code. Section 8 presents calculated waveforms which are in good agreement with experimental waveforms. The waveforms show simultaneous lasing on many transitions. The various spikes and humps are calculated and can be seen to be due to relaxation oscillations and changes in gain as the populations change (temperature was held constant). The agreement between calculated and experimental waveforms allows us to use the code to interpret the physical phenomena involved in the laser. All of the major features of the laser pulse are explained in terms of the results of the code. The code 
shows details such as populations and inversions which are very difficult to measure experimentally. As a result the code is extremely useful in illuminating the underlying physical phenomena. The calculations show that many significant phenomena can only be explained by a model which includes rotational non-equilibrium and accurate laser equations.

To complete the study calculations were done varying a number of parameters. These are presented in Section 9. Variation of the rotational relaxation rate showed the best agreement between experimental and calculated waveforms when using a rotational relaxarion rate based on estimates of the physical phenomena involved. After this work was completed Pezerson, Lindquist and Arnold (1974) published the results of a direct measurement of the rotational relaxation rate. Their rate was a factor of 2 higher than the estimate used for this work ( 5 times gas kinetic collision frequency). Calculations using an extremely large rotational relaxation rate are consistent with models that assume rotational equilibrium. The code was unable to explain the experimentally observed decrease in energy at pressures above 100 Torr, but this is probably due to electric discharge phenomena which were not modeled in the code. Variation of other parameters gave results which were predicted by the analytic model. 
One appendix is worthy ol note. Appendix $t$ pives il simple model for rotational rolaxiltion hased on dipolc-abipols. interactions. The model appeiss to be qualitatively, tut unt quantitatively, correct. 
2. THE SPECTROSCOPY OF HYDROGEN FUORIDE

\subsection{Introduction}

Before analyzing the dynamics of the $H F$ laser, it is useful to devote a few paragraphs to a discussion of the spectroscopy of $\mathrm{HF}$.

The HF laser operates on vibrational-rotational transitions all lying within the electronic ground state. This is distinctly different from atomic lasers which operate on electronic transitions. The output of an $\mathrm{HF}$ laser is dramatically different from that of a typical atomic laser. The atomic laser usually operates on one or a very few lines in the isible or near-infrared ( 1 micron or shorter wavelength). Because vibrationa1-rotational levels are not equally spaced the HF laser may lase on several out of nearly 100 possible transitions; with wavelengths in the infrared, near tinree microns.

\subsection{Vibrational-Rotational Spectrum}

Hydrogen fluoride is a heteronuclear diatomic molecule and therefore has a fairly simple specrrum. There is a single vibrational degree of freedon. In the BornOppenheimer approximation the two nuclei move in a central force potential. If this potential is expanded in a Taylor series about the minimun point, $r_{0}$, the leadinf, term is proportional to $\left(r-r_{0}\right)^{2}$. This is an harmonic: oscillatom potent iat with quanrum enerpy lavols piven by 


$$
E_{v}=(v+1 / 2) h \omega_{v}
$$

Where: $v$, an integer, is the vibrational quansi un number; the I requency, $\omega_{v}$, is a Funci $i$ on of the masses and the force constant. The vibrational levels of a harmonic oscillator are evenly spaced. Since the internuclear potential is not exactly a parabola, cubic and hipher terms in the Taylor series must be included. This leads to terms proportional to $(v+1 / 2)^{2},(v+1 / 2)^{3}$, etc., in the energy levels. In the HF spectrum these anharmonic terms are significant.

A molecile can also rotate. The simplest model for the rotational motion is a rigid rotor. In this model the; wo nuclei rotate about their center of mass with constant: internuclear distance. The quantum energy levels of a rigid rotator are

$$
E_{J}=\left(\hbar^{2} / 2 I\right) J(J+1)
$$

where $\mathrm{J}$ is the moment of inertia, and $\mathrm{J}$, an integer, is the rotatij onal quantum number. There is an additional magnetic. quantum number $\mathrm{m}$. The rotating charge distribution has a magnetic moment, so if a magnetic field is applied the direction of the rotation is also quantized. If the rotational quantum number is $\mathrm{J}$, there are $2 \mathrm{~J}+1$ allowed orientations or the angular momentun vector. In the absence of the magnetic rield each rotational state can be considered $(23+1)$-fold degenerate. This is analogous to angular: momentum in electronic spectra except that there is no 
$1 / 2$ integer spin. (We neglect nuclear hyperfine structure.) This $(2 J+1)$-fold degeneracy is important in calculating partition functions and equilibrium population distributions. The rigid rotor model is not accurate because at higher values of $J$ the centripetal forces increase the internuclear distance. This leads to terms in $J^{2}(J+1)^{2}$ and higher powers. There is also an interaction with the vibrational motion. At higher vibrational energies the nuclei spend more time at large separation and this affects the rotational motion through increased moment of inertia. When all of these effects are included, the energy of a state with quantum numbers $\mathrm{V}$ and $\mathrm{J}$ is given by

$$
\begin{aligned}
& E_{v, J}=\omega_{e}(v+1 / 2)-\omega_{e} X_{e}(v+1 / 2)^{2} \\
& +\omega_{e} Y_{e}(v+1 / 2)^{3}+\ldots+B_{v} J(J+1) \\
& \quad-D_{v} J^{2}(J+1)^{2}+\ldots
\end{aligned}
$$

The notation used in equation 2.2 is that used by Herzberg (1950). In principle, the constants in equation 2.2 can be calculated quantum mechanically from first principles. In practice, accurate spectra are measured and ritted to equation 2.2 to give the constants. Once this is done equation 2.2 can be used to interpolate and extrapolate to new levels. These constants are also useful for tabulating the spectra of molecules, since the spectra can be calculated from the constants. Such a table is given in Herzberg (1950). The constants can also the used to calculate thermodynamic tables by means of a partition function. 


\subsection{Selection Rules}

The spectral lines arise when the molecule makes a transition from one energy level to another. Certain selection rules limit the number of possible transitions. For a harmonic oscillator the vibrational transition selection rule is $\Delta \mathrm{V}= \pm 1$, with +1 representing absorption and -1 representing emission. The rotational selection rule is

$$
\Delta \mathrm{J}=\mathrm{I} I, 0 \text {. }
$$

Both rules operate simultaneously so that in the cars of emission $\Delta v=-1$ and $\Delta \mathrm{J}=-1$, D, or 1 . By convention these are denoted as $R, Q$, and $P$ branch transition: , When the electronic ground state is a singlet the $Q$ branch is forbidden. Thus HF has only $P$ and $R$ branch transitions in its vibrationalrotational spectrum.

If the potential is anharmonic, as in che case of HF, transitions with $|\Delta v|>1$ are also allowed but are much less probable than those with $|\Delta v|=1$. The rotational selectional rule, $\Delta \mathbf{J}= \pm 1$, is valid in any case. These are electric dipole transistion selection rules; there are different selection rules for electric quadrupole and magnetic dipole transitions. These are unimportant in the HF laser because the transition probabilities are small.

In the $\mathrm{HF}$ laser we are then concerned only with $P$ and $R$ branch electric dipole transitions. The observed spectra from HF laser generally involves only P-branch transitions. It is difrtcult to maintain an R-branch population inversion becausr: of the Boltaman factor in population distributiuns, Lasing on the P-branch transitions tends to deplete the R-branch inversion. 
It is worth notitg that the fif laser operates on allowed transitions with lifetimes on the order of $10^{-3}$ seconds. Most visible lasers operate on transitions which are forbidden because it is too hard to pump allowed transitions of $10^{-8}$ seconds. Small perturbations of the electronic states give the forbidden transitions a finite probability with lifetimes in the range of $10^{-3}$ to $10^{-5}$ seconds. The lifetime of $\mathrm{HF}$ is relatively long Frimarily because the lower frequency drastically reduces the density of modes into which the molecule can emit. However, since the transition is fully allowed the stimulated emission cross section in $\mathrm{HF}$ is large, leading to high gain lasers. The visible laser tends to have a smaller stimulated emission cross section because the transition is largery forbidden.

Tables of the spectra of $\mathrm{HF}$ are given in Appendix A. Because of anharmonic terms and the vibrational dependence of the rotational constants the lines are nearly all separated by more than the Doppler line width. The few lines which overlap do so by accident, not because of natural periods in the spectrum.

The standard notation for vibrational-rotational lines is to give the branch and rotational quantum number in the lower vibrational level, c.g. P3. hie shall also use the notation V2-I to indicate a cransition form $v=2$ co $v=1$. Thus a V2-1 P3 transition comects the states $v=2, J=2$ and $v=1, J=3$. 
The calculations presented here were part of a program involving both experiment and theory. The experiments were performed by Dr. Richard Pearson and co-workers, and many of the experimental results have been pulslished (Pearson, et al. 1973a, 1973b). There was consistent comnunicution between myself and Dr. Pearson, and he has kindly made his experimental notet ooks availa'ble. This section will briefly review the experimental work, both the published material and some that has not been published.

\subsection{Apparatus}

A schenatic diagram of the experimental apparatus is showti in Fig. 3.1. The electric-discharge laser tube consisted of a row of 51 aluminum electrodes - paced $1.0 \mathrm{~cm}$ apart in a thick-walled, 3/4-in-ID Teflon tube $65 \mathrm{~cm}$ long. Each e]ectrode is ballasted with a $1000-\Omega$ resistor that discherged to a $1 / 4-i n$ diam aluminum bar $50 \mathrm{~cm}$ long, across a $1.5 \%-\mathrm{cm}$ discharge gap. The tube had Brewster angle BaF windows held in pizce by a stainless-steel window holder. Viron G-rings wero used to seal windows and electrodes. The laser tube was filled with premixed gases from a stainlessstee 1 and Teflon vacuum line.

The laser cavity had a high-reflectivity goldcoated rear mirror with a $10 \mathrm{~m}$ radius of curvature and a flat outpit mirror. Experiments using output mirrors with

\footnotetext{
Reference to a company or product name does not imply approval or recommendation of the product i.y the University of California or the U. S. Atomic Energy Com ission to the exclusion of others that may be suitable.
} 
$90 \%, 50 \%, 8 \%$, and $0 \%$ reflectivity showed that maximum lasing energy was obtained with a $\mathrm{BaF}_{2}$ flat ( 8 percen measured). The energy delivered to the laser was calculated from measurements of the current and voltage across the discharge as a function of time. The energy out of the laser was determined by a cone ballistics laser calorimeter. A second detector and a PE Model E-1 monochromator were used to study the pulse shape of individual speccal lines. Both detector outputs were displayed in a dual-beam oscilloscope to give relative timing. Electrical-energy input could be varied by adjusting resistor $R$ ( $F i g .3 .1$ ). For most experiments a resistor was not used.

At low pressures ( $<15$ Torr), the discharge current pulse terminated before any lasing began; at tigher pressures ( $>25$ L r) lasiag began before the discharge pulse had ended and persisted well beyond cessation of current flow A. 10-30 min, evacuation of the laser tube was found to be necessary between successive firings and was used to avoid a reduction of laser output from residual explosion priducts of the previous firing.

Occasionally, a permanent reduction of laser output was observed, and when it reached 10 percent the laser was dismantled and cleaned. Restoration of the output to a standard $\mathrm{NF}_{3}-\mathrm{H}_{2}$ mixture was achieved by washing the laser tube with aqueous detergent, then rinsine with water, methanol, and acetone. The tube was then evacuated overnight. The gases used werc the highest grade conmercially available. 


\section{2 havelorms}

A particular seriess of experine'nt s was chosed for comparison with calculations. The series was perlorned by Major Gordon L. Hermam, USAF, during February, 3971. The series involved several fundred laser shots at a 5:1 ratio of $\mathrm{NF}_{3}: \mathrm{H}_{2}$. Most shots were at 15 Torr total pressure with about $20 \%$ at 60 Torr. This series was selected because each shot recorded the waveform for one single transition along with the total laser waveform. The total pulse energy Wus álso measured by a calorineter.

The waveforms for individual transitions obtained from experiment are shown in Fig. 3.2. These were obtained in experiments at 15 Torr total pressure with the $\mathrm{NF}_{3}: \mathbf{H}_{2}$ ratio about 5.2. The waveforms shown are the only ones observed, except that a very weak V3-2 P3 was seen in one case. The V1-0 P5 is apparently missing due to coincidence with a water vapor 2 ine. Since part of the laser cavity was open to the laboratory atmosphere, the absorption by water vapor probably prevented lasing on the P5 1ine. Searches were made for the V3-2 P5, V2-1 P 7 and V1-0 P8 1ines, and they were not found within the limits of experimental sensitivity. The sensitivity was limited by electrical noise which created noise equal to 5 to $10 \%$ of the height of the V2-1 P5 cransition. Thus it is not clear whether the missing transitions are completely missing or merely too weak to be detected. 
The amplitude scale of the waveforms is arbicrary because they were obtained by splitting off an unknown fraction of the beam. In the case of the individual transitions the light was also passed through a monochromator which had uncalibrated losses. The heights are proportional to the signal recorded by the oscilloscope, but there may still be errors in the relative scale between individual transitions because they were of ten made several days apart, and 1 ient collection efficiencies could change due to changes in alignment or systematic changes in laser operation.

The waveforms shown in Fig: 3.2 were replotted by hand from oscilloscope photos. In that process the waveforms were considerably smoothed as shown by comparison with Fig. 3.3. Some of the wiggles in Fig. 3.3 represent small perturbations in the true waveform and some represent electrical noise due to ringing in the pulsed power supply. Both types of variation were smoothed out in the process of replotting. This must be kept in mind when comparing with calculated waveforms. The general outline of tho experimental waveform, including multiple humps has been prei arved.

There is also a problem of experimental reproducibility. The samples chosen were beljeved to be representative, but there were signiricant variations in experimental waveforms. There was little variation for P3 and P4 except occasional changes in amplitude. These were usually accompanied by other anomalies in the laser pulse. When the laser was restored to its normal operating condition, there waveforms returned to normal. Nearly half the experimental waveforms were v2-1 P5. 
while thr gonoral shape was lat rly consistent, the relative height:s of the spikes varied Irom : ince to time. The Po and P7 waveforms were more erratic. They sometimes looked like the $[5$, they were of ten as shown, and sometimes disappeared encirely. They seemed to be particularly sensitive to some variacion in the laser, but the source is not clear.

Figure 3,4 shows a similar set of waveforms where the: total pressure was 60 Torr. haveforms for a number of transitions are missing. Those that appeared at 15 Torr wore skipped in this experimental series. The P8 and P2 Lrälsitions were checked and were not lasing, or were not deteclảble.

Another phenomenon is significant. At 15 Torr the total laser energy, as measured by calorimeter, was about ! millijoulc. At 60 Torr we would expect the energy to be 4 millijoules. Instead, the total energy was only about 2 millj joules. This discrepancy will be discussed later. In addition to recording the total laser energy, the partitioning of this energy among the various transitions hics been calculated, and is shown in Table 3.1. The fractions were calculated by graphically integrating the waveforms of Fig. 3.2. The values given should be considered approximate, since the pulse heights depend on pulse to pulse reproducibility, which was not very good,

3.3 Dissociation of $\mathrm{NF}_{3}$ by the Electric Discharge

There is good reason to expect the output of the $\mathrm{NE}_{3}+\mathrm{H}_{2}$ laser to rise in a roughly linear fashion with 
increasing pressure. It will be shown in Section 4 that the total laser output energy is proportional to the initial number of flourine atoms created by dissociating $\mathrm{NF}_{3}$. If the fraction of $\mathrm{NF}_{3}$ dissociated is independent of the pressure, the output energy should rise linearly with increasing pressure. Figure 3.5 shows that the output energy increases for pressures up to about 80 Torr and then drops off at higher pressures. This could be explained if the fraction of $\mathrm{NF}_{3}$ which is dissociated decreases at higher pressures. We will examine the experimental data for the discharge to see if there is evidence for this hypothesis. The experimental data is from Pearson, et al. (1973a, 1973b); and from unpublished data of $G$. L. Hermann.

The source of $\mathrm{F}$ atoms is dissociation of $\mathrm{NF}_{3}$ by the electric discharge. The physics of this process is quite complicated, but we can make some observations based on experimens.al data. We note from Fig. 3.6 that the current decreases slowly with increasing pressure, and we note from Fig. 3.7 that $E / P$ is approximately constant at higher pressures. Actually, Fig. 3.7 shows $V / P d$ where $V$ is the voltage, $d$ is the spacing between electrodes, $P$ is the pressure, and $E$ the electric field. Figure 3.8 shows that the voltage remains finite in the limit of zero pressure. If we ascribe the finite voltage to a potential drop near the electrodes, then E/P is nearly constant over the entire experimental range. The simplest model for a discharge assumes that an electron gives up most of its kinetic energy in an inel.stic collision (von Engel, 1956), and then obtains an average kinetic energy at by the 
Lime it has another inelastic collision. The mean-free path, $\lambda$, is proportional to $1 / \mathrm{P}$, so if $\mathrm{E} / \mathrm{P}$ remains constant the average: electron energy at the time of an inelastic collision remiatis the same as the pressure varies. The inelastic scattering can excite the molecule, dissociace it, ionize it or attrich the electron. The physics of these processes is quite complicated, but if the energy of electron impact remains the same the probabilities for these processes should remain he same. The fact that E/P remains constant as pressure increases means that the probabilities will remain the same, but the number of jnelastic collisions will be proportional to the pressure, $P$. Thus the number of $F$ atoms produced by dissociation will also be proportional to $P$ if the current remains thr. same. We have seen, however, that the current decreases with increasing pressure, so there may be a pressure where the number of $F$ acoms is a maximum. This may explain the rall-of $f$ in energy and power at higher pressures. The equivalent electrical circuit of the pulser and discharge is shown in Fig. 3.9. The pulser is equivalent to a voltage source, $V_{S}$, and a source impedance, $R_{S^{*}}$ (This is an accurate representation because the pulser is constructed from transmission line, rather than a conventional capacitor.) The output of the pulser is connected to a terminating resistor, $\mathrm{R}_{T}$, to prevent reflections of the pulse which might damage the pulser. The laser discharge with a series resistor, $R_{D}$, is also connected across the output, in parallel with the terminating resistor. The 
discharge is represented as a voltage source, $v_{D}$; and the resistor $R_{D}$ is in two parts, a current limiting resistor designated as $R$ in Fig. 3.8 and an equivalent 20 ohms made up of 1000 ohms in series with each of the 51 pins in the discharge tube.

We can find the discharge current by analyzing the equivalent circuit. Clearly,

$$
\begin{aligned}
& I=I_{D}+I_{T} \\
& I_{T} R_{T}=I_{D D} R_{D}+V_{D} \\
& V_{S}-I_{S} R_{S}-I_{T} R_{T}=0
\end{aligned}
$$

In the experiments $R_{T}$ was designed to equal $R_{S}$. Solving Eqs. 3.1 to 3.3 with $R_{S}=R_{T}$ gives the discharpe current

$$
I_{D}=\frac{\vartheta_{S}-2 V_{D}}{R_{S}+2 R_{D}}
$$

The factors of 2 arise because of the terminating resistor which carries substantial currents at larger values of $v_{y}$. The nominal value of $V_{S}$ is $160 \mathrm{kV}$ with $R_{S}=80$ ohms. If we obtain $V_{D}$ from litis. 3.8 with $R_{D}=20+R$, the calculated discharge currents agree with Fig. 3.6.

We can now estimate the total number of $\mathrm{F}$ atoms created by the discharge. As shown above, the number of $F$ atoms produced by one electron in the Uischarge is aV if E/P remains constant. The value of a can be determined empirically. The number of electrons is proportional to the current, so the number of Fluorine atoms, $f_{0}$, is proportional to $v_{v} l_{1}$. The values of $V_{v}$ lrom fig. 3.8 can be represented by 


$$
v_{i}=v_{G}(R)+\gamma P
$$

with $\gamma$ about $200 \mathrm{~V} / \mathrm{torr}$. The constant, $V_{0}(R)$, is independen' of the pressure but is a function of thr series rosistance $R$. for a lixul value of $R$,

$$
\left.F_{0}-\eta\right]_{11} v_{n}=\eta \frac{v_{S}-2 v_{0}-2}{R_{S}+2 R}\left(v_{0}+T P\right) \quad(3.6)
$$

1f we neglect $v_{e}$ compared to $v_{S}$ ind $\gamma_{P}$

$$
r_{0}=\eta \gamma \frac{v_{S}-2 r P}{R_{S}+R}
$$

when $F_{0}$ is plotted against $P$ the curve is a parabola with $a$ maximum at $\gamma P=V_{S}$. $1 f V_{S}$ is $160 \mathrm{kV}$ ard $\gamma 200 \mathrm{v} / \mathrm{torr}$, this maximum occurs at about 800 torr. The mixtmum of Eq. 3.6, which includes $v_{e}$, occurs at about 600 corr. This is an order of magnitude larger than the experinental maximum. ligure 3.10 shows laser output enterby as a function of discharge energy. This shows that the fall-off in laser pulse (merg) is not solely due to passing the discharge energy peak. It appears that other phenomena are important in determining; the pressure for maximum laser energy.

This is reinforced by experimental data for mixtures of $\mathrm{SF}_{6}+\mathrm{H}_{2}$ where the maximum laser energy was obtained at about 120 torr and the energy was nearly four times the maximum obtained with $\mathrm{NF}_{3}+\mathrm{H}_{2}$ (Fig. 3.11). The discharge energies were very nearly the same, as is shown in Fig. 3.12. Apparently the discharge creates more $F$ atoms in $S_{6}$ than it does in $\mathrm{NF}_{3}$. 
Apparent:1y the non-uni form fields dus ro the geometry also have an influnice. The discharge goes from pins perpendicular to the lasor axis to a cylindrical wire parallel to the laser axis. Flectroles with these shapes cause the electrie field to le highly non-uriform and add adelixional compl contions co undersianding tne physirs involved. The distriburion of laser intensity relative to the reectedes is shown in Fig. 3.13. The distriluston was inrasured two neters from the laser outgut intror with an array of proelectric detectors. From this distribution the size of the laser beam woist was estimatod. It is in aprrencont with the stze determined by spots burned into film near the laser output mirror. There is some evidence that: the spor narrows by 20 to $50 \%$ ar pressures over 200 torr. This may result in reduced laser energy and power. 


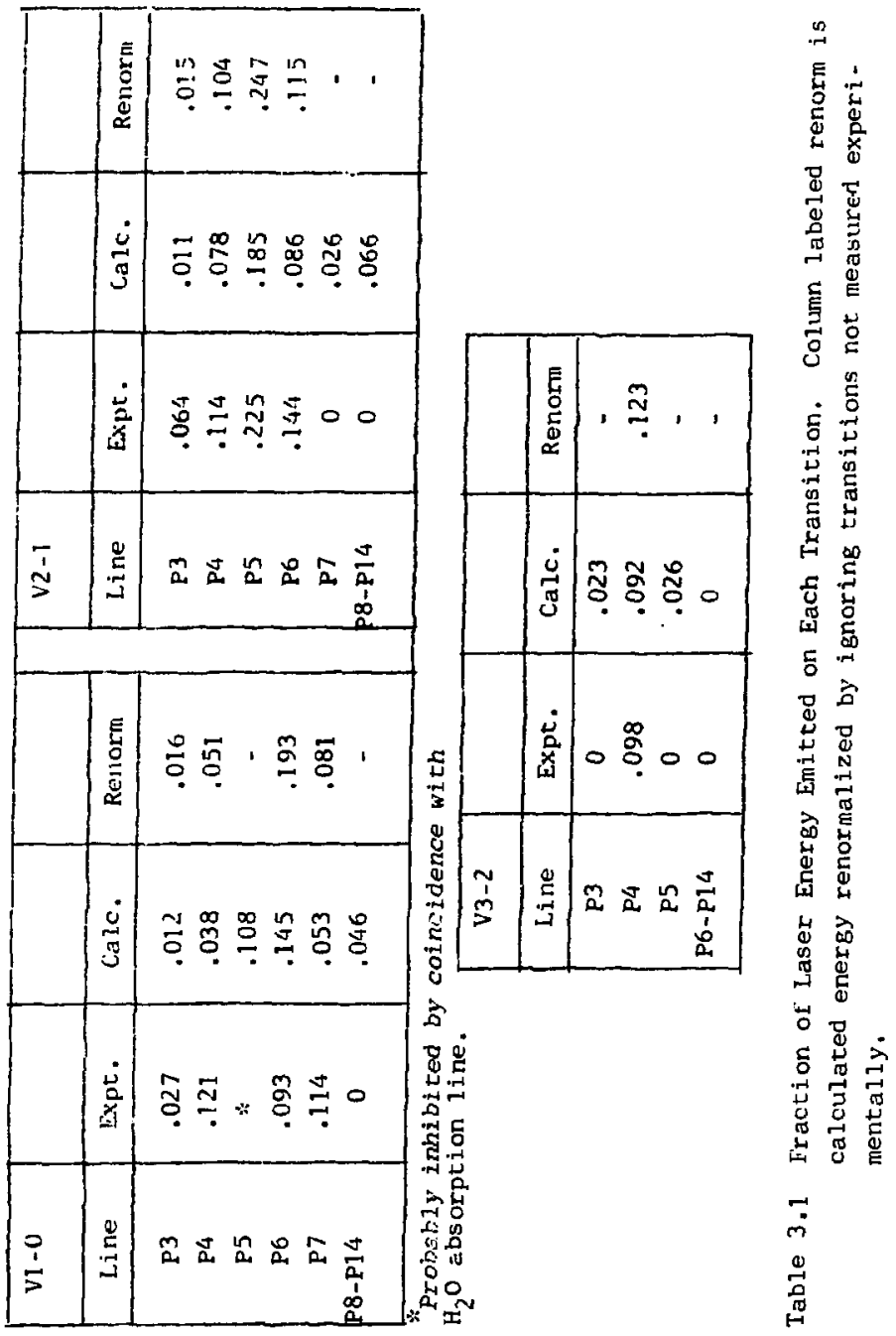




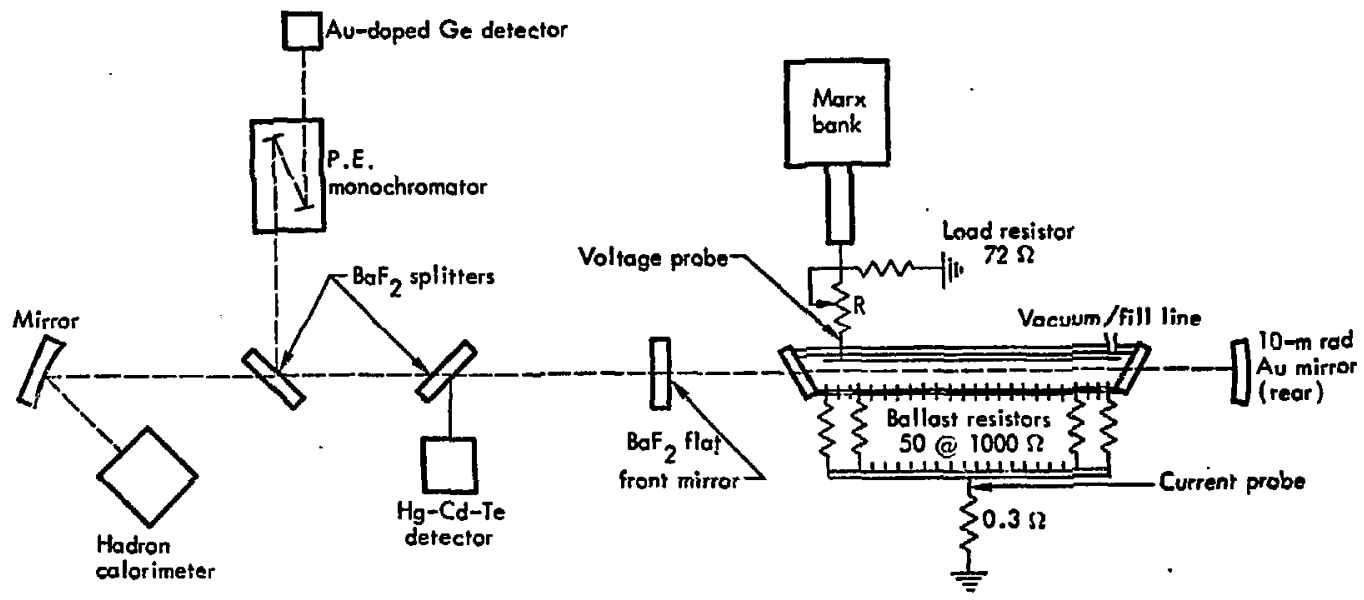

Fig. 3.1 Schematic of apparatus used for $\mathrm{NF}_{3}-\mathrm{H}_{2}$ chemical laser experiments. 

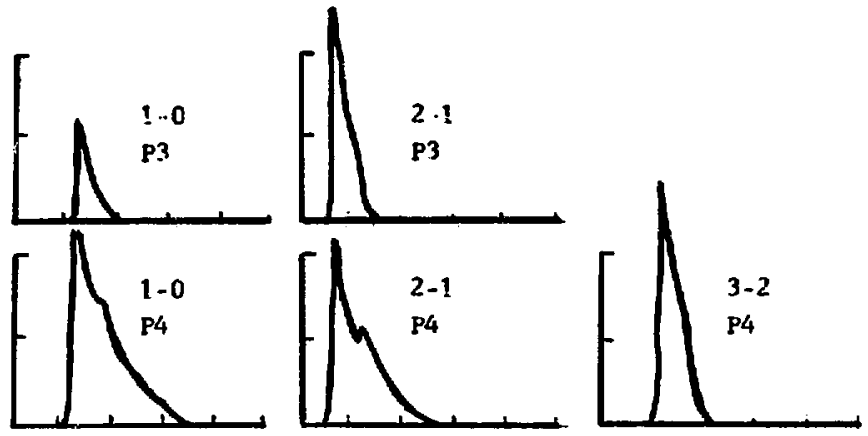

Missing:

coincides with bi.,O absorption line.
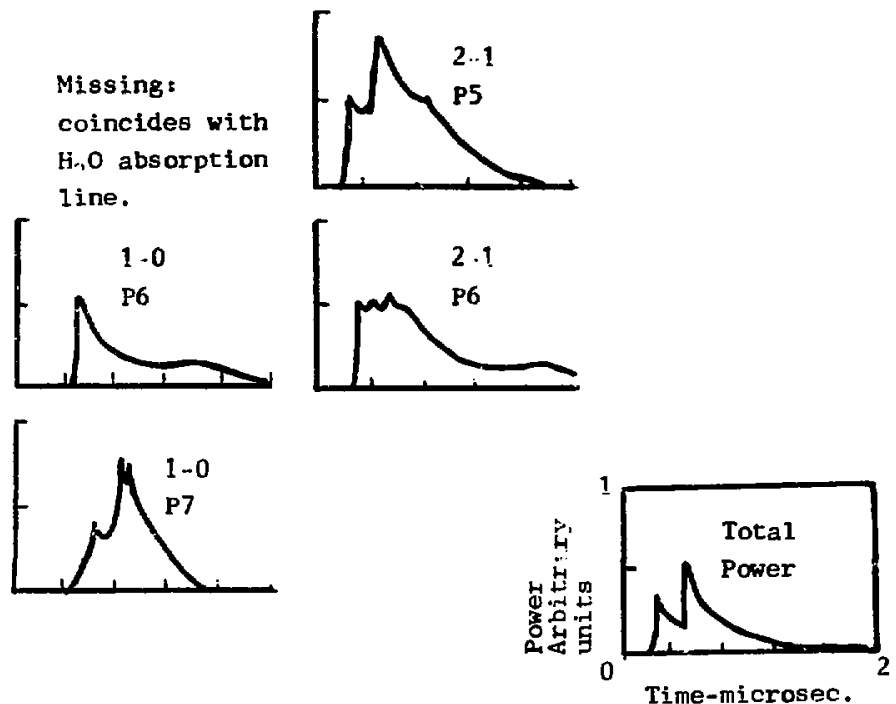

Fig. 3.: Experinent..l wiveforms for sndividual laser transi-

L. ons. Tot:1 pressure wis 1.5 Torr with $\mathrm{NF}_{3}: \mathrm{H}_{2}$ ritio 5.' Peak totill power is shout :.kW. 


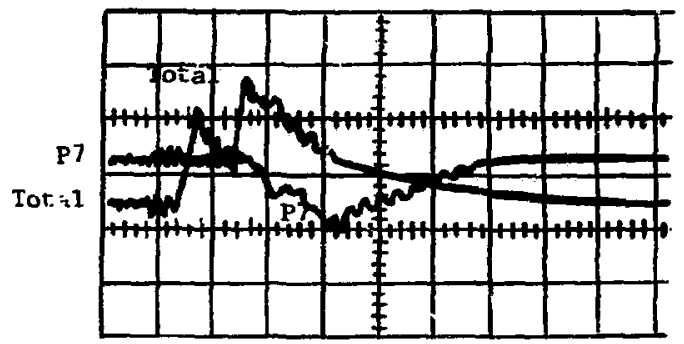

Fig. 3.3 Osc:.1loscope $t r$.ce show: ns c:al laser pulse and $V^{4}-0$ P7 w vectorm. Hor: on 31 sweep is 0.2 microsec $\mathrm{cm}$. Vertacil scale is uncalijzat. 

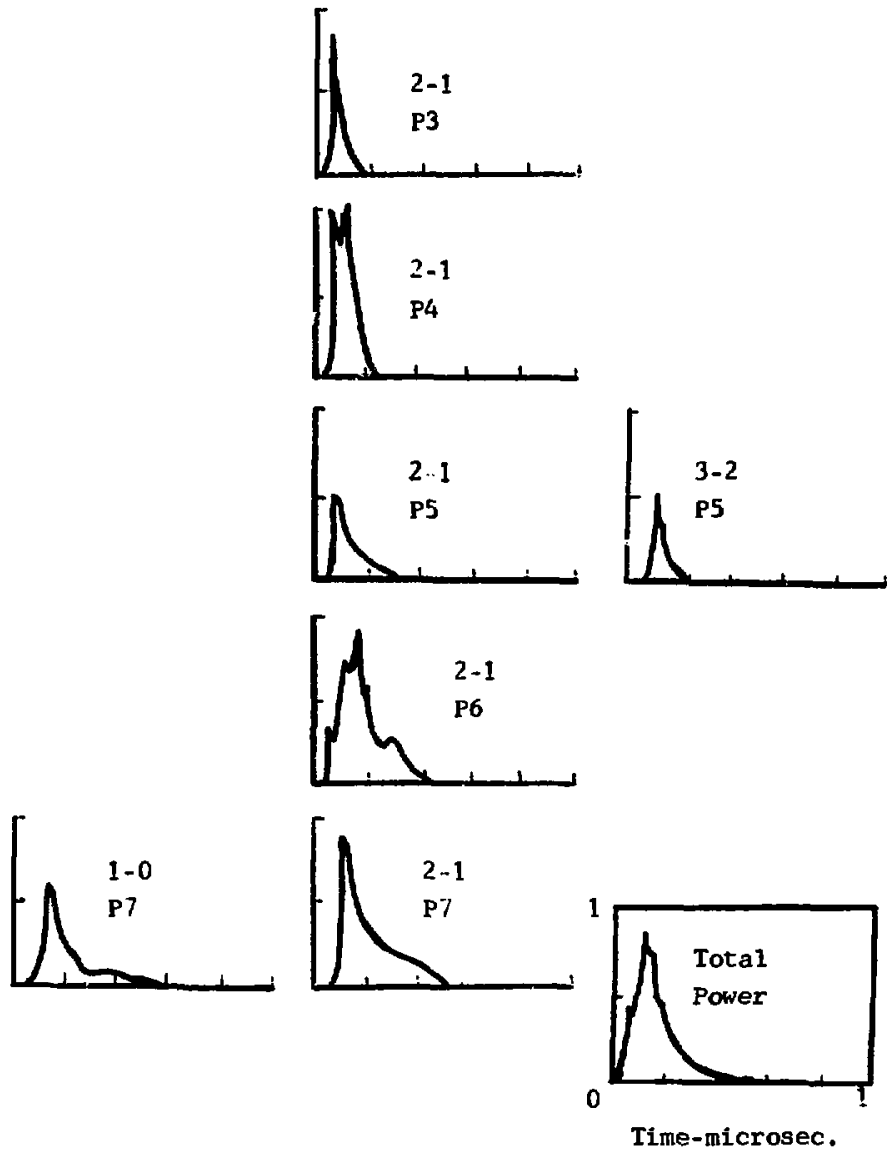

Fig. 3.4 Experiment:1 wryeforms a: 50 Torr total pressure W:.h $\mathrm{NF}_{3}: \mathrm{H}_{2} \mathrm{r} \cdot: \mathrm{O}$. 5.?. Ahsence of several wavetorms is dive to in incomplete search. 


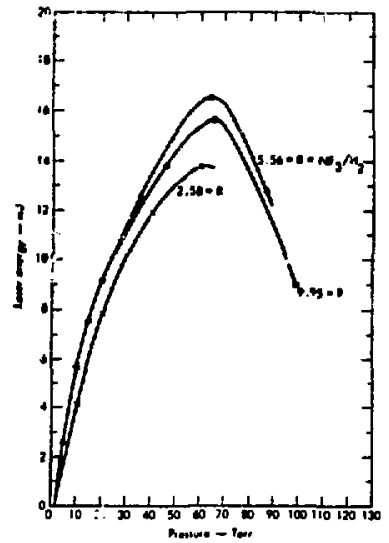

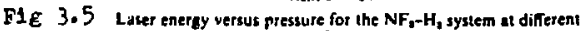
reactant ratios: $R=N F_{V} / H_{\text {, }}$.

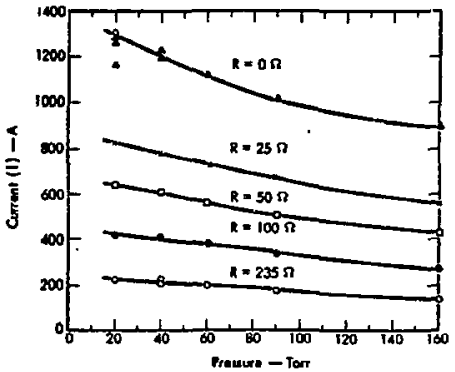

Fig. 3.6 Discharge current versus pressure for $\mathrm{NF}_{2} / \mathrm{H}_{1}=5.4$, using , current-limiting rexistors and a discharge time of $70 \mathrm{~ns}$.

Reproduoed by permisston from Fearson, et. al. (1973a end 1973b) 


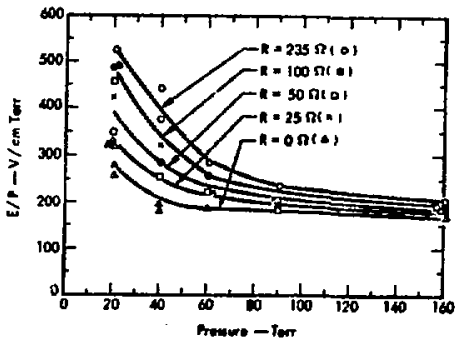

FiE. 3.7 E/P (valts/centimeter tort) of the discharge vesus ptessure for $\mathrm{NF}, \mathrm{H}_{1}=5.4$. using eurrent-Jimiting resistors and $\mathrm{a}$ dicharge time of $70 \mathrm{~ns}$.

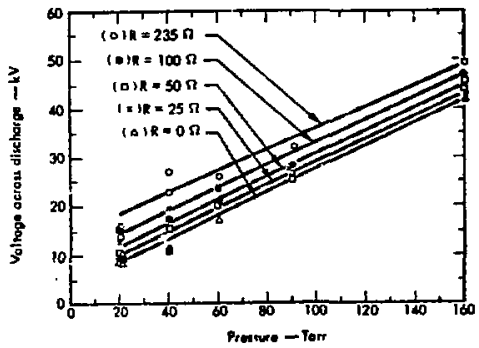

Fir. 3.8 voltage actoss laser tube (not including hallast resistors) versus pressure for $\mathrm{NF}_{3} / \mathrm{H}_{2}=5.4$, using current-limiting resistors and a discharge time of 70 ns.

Reproduced by permlesion from Pearson, et. el. (1973a). 


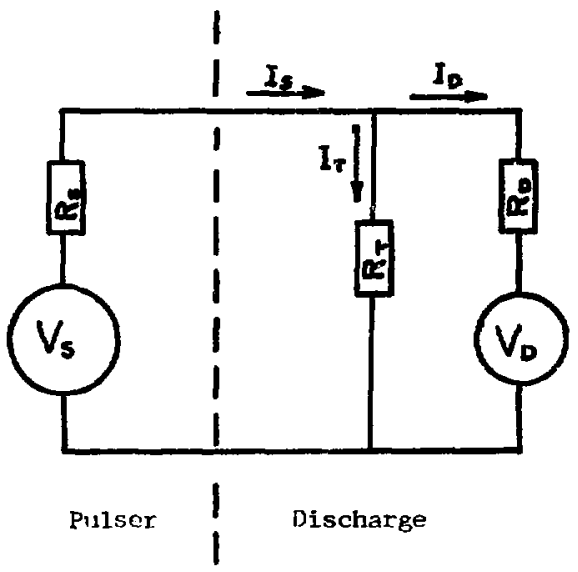

Fig. 3.9 Equivilent circu't of Pilser ind tischirfo in the Liser Tibe. 


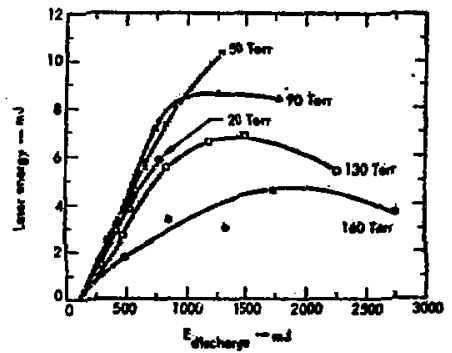

FiE. 3.10 Laser entrgy versus energy deposined in the ces from the the

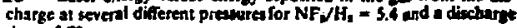
jime of $70 \mathrm{~m}$.

Beproduced by van mission from Pearson, et. al. (1973a). 


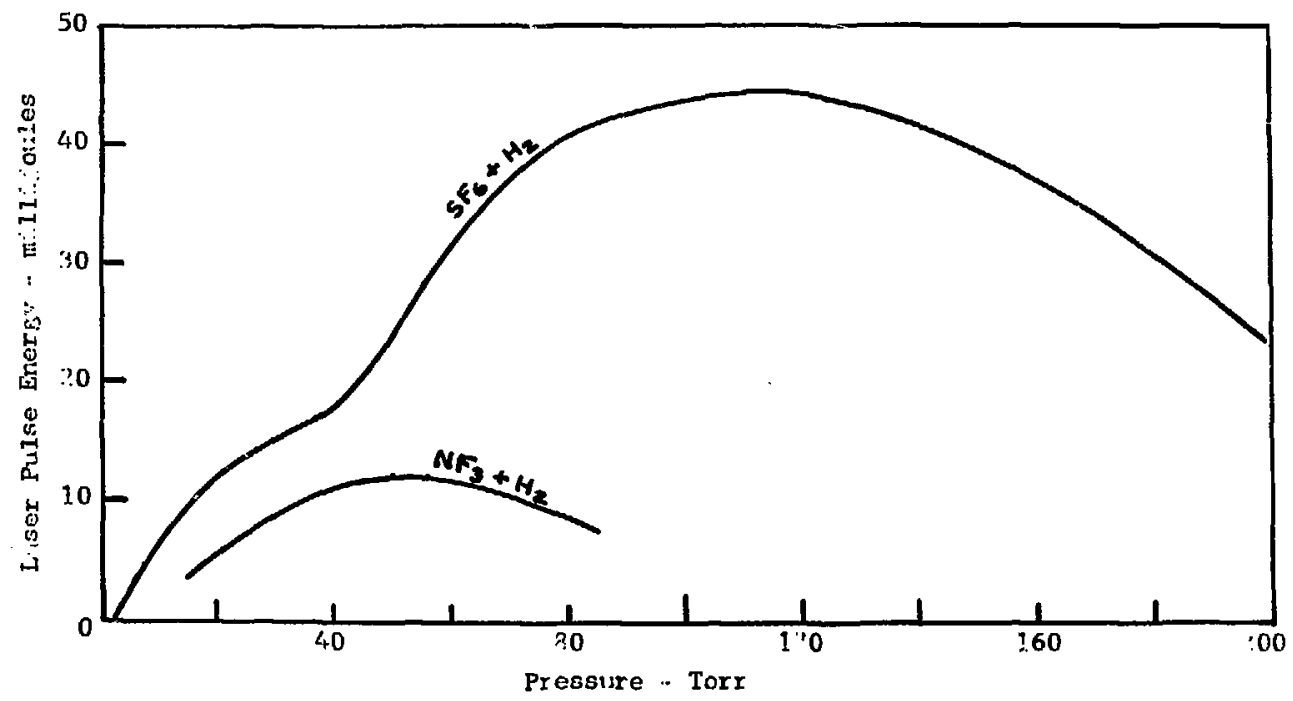

F: 3,11 V:r: $:$ on of 1 -cer pulse eners: with prescire for mixures of $\mathrm{SF}_{6}+\mathrm{H}_{2}$ nd $\mathrm{NF}_{3}+\mathrm{H} \cdot$. 

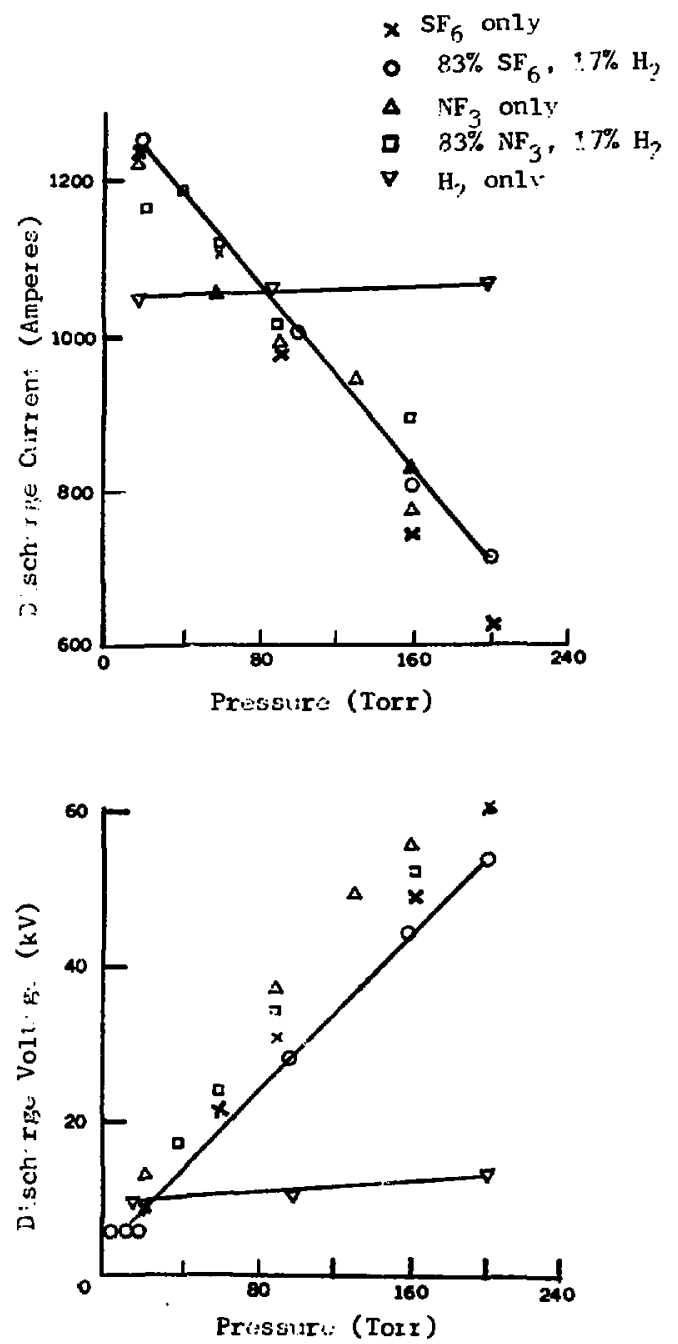

F* E. 3.1? “sch.rfy: rurrun! nd "olthe for several mixtures of $\mathrm{SF}_{5}, \mathrm{IF}_{3}$ and $\mathrm{H}_{1}$. 


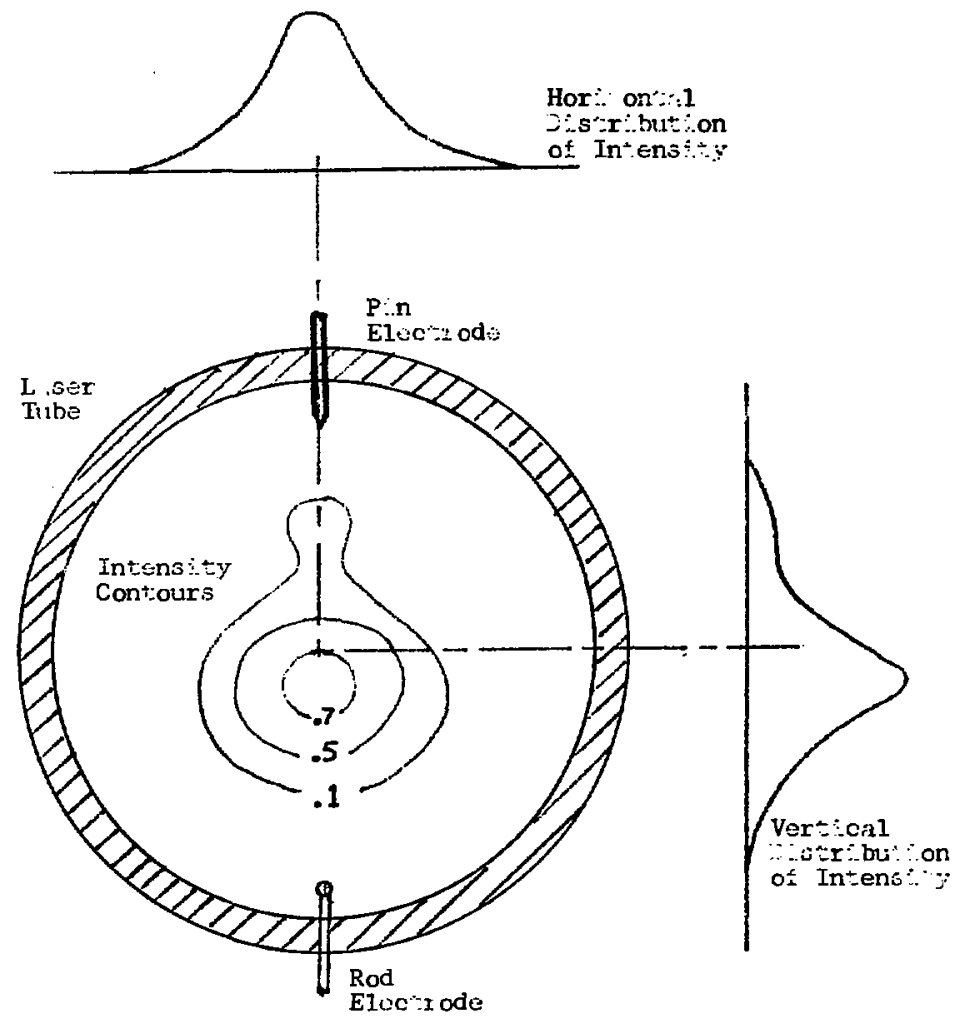

Fig. 3.13 Approxim te d s'.r.but:on of Liser intenst. us ing ?0 Torr $\mathrm{SF}_{6}$ plus 0 Torr $H_{?}$. 
4. AN ANALYTIC MODEL OF THE CHEMICAL LASER

Many of the basic features of the HF chemical laser can be understood in terms of a simple analytical model. Numerical calculations are required to understand many details but the general pulseshape and pulsewidth can be obtained from the analytical model. The analytical model consists of a steady-state laser model and simplified treatments of the chemical kinetics.

\section{4,1 The Steady-State Laser Model}

We deal first with the laser model. It is well

known that if 1 ight of the appropriate wavelength is passed through an inverted medium the intensity wil1 be

$$
I=I_{0} e^{2 \sigma \Delta N}
$$

where $\Delta N$ is the population inversion, $\sigma$ is the stimulated emission cross section, $z$ is the distance traveled, and $I_{0}$ is the intensity at $z=0$. We wish to analyze a laser cavity containing an inverted medium of length $L_{g}$, one perfectly reflecting mirror and a second mirror with reflectivity o. Light which has just been reflected from the second mirror will be amplified as it passes through the inverted medium. It will then be reflected and amplified a second time. Finally, it is reflected by the second mirror. If $I_{1}$ is the intensity at that point, then $I_{1}=I_{0}^{p} \exp \left(2 I_{g} \sigma \Delta N\right)$. To maintain a steady state $I_{1}$ must very nearly equal $I_{0}$ ' so we have $\rho \exp \left(2 L_{g} \sigma \Delta N\right)=1$. This can be rewritten 


$$
N=\log (1 / 0) / 2 \sigma \mathrm{Lg} .
$$

Physically, the population inversion adjusts itself until the gain equals the loss, including output, in one round trip through the cavity. This can occur because an increase in light intensity can deplete the inversion. The intensity then adjusts itself so tr.t it depletes the population inversion which compensates tor the increase due to pumping. This steady-state intensity, $I_{S}$, is easily calculated. Consider a laser with $\mathrm{N}_{1}$ molecules (atoms) in an upper state and $N_{0}$ in a lower state, so that $\Delta N=N_{1}-N_{0}$. Assume that only the upper state is pumped at a rate $s_{1}$ by some mechanism such as a chemical reaction. The rate of creation of photons by stimulated emission is $\sigma I_{S} \Delta N / \hbar \omega_{1}$, and the rate equations for the populations are

$$
\begin{aligned}
& \mathrm{dN}_{1} / \mathrm{dt}=\mathrm{s}_{1}-\sigma \mathrm{I}_{\mathrm{s}} \Delta \mathrm{N} / \mathrm{h \omega} \\
& \mathrm{dN}_{0} / \mathrm{dt}=\sigma \mathrm{I}_{\mathrm{s}^{\Delta N} / \mathrm{L} \omega .}
\end{aligned}
$$

Since $\Delta N$ is constant in the steady state,

$$
\mathrm{dN}_{1} / \mathrm{dt}=\mathrm{dN}_{2} / \mathrm{dt}
$$

and

$$
I_{S}=s_{1}\{1 \omega / 20 \Delta N \text {. }
$$

Equations 4.2 and 4.3 also imply that the output power plus other losses equal one half the pumping rate into the upper state, because in the steady state $I_{S}$ remains constant and the number of newly created photons, a $S^{\Delta N / \hbar t h}$, must equal the number lost. If the lower level wrere also pumped at a rate $s_{0}$ the output rate would be proportijonal to $1 / 2\left(s_{1}-s_{0}\right)$. The principal conclusion reached in this discusion is the 
lace thal the oulput power is proportional to the pumping, rales as lonp as the laser operates with fain cquat to loss. Thercfore, the problem of determining the steady-state power output reduces to the problem of determinine, the pumping rate. We can generalize this to the case of the HF laser where several vibrational levels are pumped. The chemical reaction

$$
\mathrm{F}+\mathrm{H}_{2}+\mathrm{HF}+\mathrm{H}
$$

pumps four vibrational levels from $v=0$ to $v=3$. Twentylive percent of the molecules go into $v=3,52 \%$ into $v=1$, and $8 \%$ into $v=0$ (Cohen, 1971). Representing these as $\mathrm{S}_{3}$ to $S_{0}$ we can write rate equations

$$
\begin{aligned}
& \mathrm{dN}_{3} / \mathrm{dt}=\mathrm{S}_{3}-\mathrm{B}_{3} \phi_{2}\left(\mathrm{~N}_{3}-\mathrm{N}_{2}\right) \\
& \mathrm{dN}_{2} / \mathrm{dt}=\mathrm{S}_{2}+\mathrm{B}_{3} \phi_{2}\left(\mathrm{~N}_{3}-\mathrm{N}_{2}\right)-\mathrm{B}_{2} \phi_{1}\left(\mathrm{~N}_{2}-\mathrm{N}_{1}\right) \quad(4.5 \mathrm{~b}) \\
& \mathrm{dN}_{1} / \mathrm{dt}=\mathrm{S}_{1}+\mathrm{B}_{2} \phi_{1}\left(\mathrm{~N}_{2}-\mathrm{N}_{1}\right)-\mathrm{B}_{1} \phi_{0}\left(\mathrm{~N}_{1}-\mathrm{N}_{0}\right)(4.5 \mathrm{c}) \\
& \mathrm{dN} / \mathrm{dt}=\mathrm{S}_{0}+\mathrm{B}_{1} \phi_{0}\left(\mathrm{~N}_{1}-\mathrm{N}_{0}\right)
\end{aligned}
$$

Steady state implies that $\mathrm{dN}_{3} / \mathrm{dt}=\mathrm{dN}_{2} / \mathrm{dt}=\mathrm{dN}_{1} / \mathrm{dt}=\mathrm{dN} / \mathrm{dt}$. Let the output power on transitions between $v=1$ and $v=0$ be $P_{0}=0 T_{S} N / K_{1}{ }_{0}$, and let $P_{1}$ and $P_{2}$ be the corresponding powers on other transitions. At steady-state $\sigma_{1} I_{S I}=B_{1} \phi_{0}$ and we get.

$$
\begin{aligned}
& P_{2}=1 / 2 x_{2}\left(s_{3}-s_{2}\right) \\
& P_{1}=1 / 3 \not \omega_{1}\left(S_{3}+s_{2}-s_{1}-s_{0}\right) \\
& P_{0}=1 / 6 \hbar \omega_{0}\left(S_{3}+s_{2}+2 s_{1}-4 S_{0}\right)
\end{aligned}
$$


All of the pumping terms, $S_{0}$ through $S_{3}$, are proportional to the rate of formation if $\mathrm{HF}$. The total power is also proportional to the rate of formation, but it is no longer one half the pumping rate as it was in the two-level case. Instead, there will be some other numerical factor.

The steady-state solution will be valid over most of the pulse, but it neglects transients at the beginning of the pulse. Detailed discussion of the transient solution will be postponed to a later section because the rate equations are nonlinear and must be solved numerically. For the present we will merely note that there is a delay from the start of pumping to the time that a threshold inversion is reached. There is a further, and perhaps longer, delay until the laser intensity builds up to the steady-state value. The intensity wi11 in fact, overshoot steady state and execute a damped oscillation about the steady state. For the moment we will neglect this and consider only the steady state. In the steady state the sum of the output power plus losses equal a fraction of the pumping rate. The HF chemical laser operates well with a low reflectivity mirror so that we can neglect losses in comparison with the output power. Thus we can find the output power once we know the pumping rate. 


\subsection{Chemical Pumping ol 1 le Hi lialier}

4.2.! Introduction

In the HF laser the pumpinf, rate is decermined by the rite at which $\mathrm{HF}$ is formed in vibrationally fxcited states. This study is primarily concerrital with lasers where reaction 4.4 is the source of $\mathrm{HF}$. The ?luorine atoms are obtained by dissoriatine, compounds such as $\mathrm{NF}_{3}$ or $\mathrm{SF}_{6}$. (See Section 3.) Thls is readily done by an electric discharge which can be complete before much of the fluorine can react. This will allow us to assume an initial density, $F_{0}$, of fluorine atoms and ignore the details of the discharge. The discharge undoubted $1 y$ creates ions and other chemical species but they seem to react on a much slower time scale than reaction 4.4 so that they can be ignored.

A second important case is when molecular fluorine, $r_{2}$, is the source of the fluorine atoms. Then the reaction

$$
\mathrm{H}+\mathrm{F}_{2}+\mathrm{HF}+\mathrm{F}
$$

is an afditional source of $\mathrm{HF}$. The reaction now proceeds as a chain where reaction 4.4 provides the $H$ atom for reaction 4.7, which then provides a new $F$ at om for reaction 4.4. This proceeds until one of the reactants is consumed or until the atoms are removed by a recombination reaction of the form

$$
\begin{aligned}
& \mathrm{H}+\mathrm{H}+\mathrm{M}+\mathrm{H}_{2}+\mathrm{M} \\
& \mathrm{F}+\mathrm{F}+\mathrm{M}+\mathrm{F}_{2}+\mathrm{M} \\
& \mathrm{H}+\mathrm{F}+\mathrm{M}+\mathrm{HF}+\mathrm{M} .
\end{aligned}
$$


These will be discussed later. Chain reactions, 4.4 plus 4.7 , give a different waveform than the single reaction, 4.4 . The waveform for reaction 4.4 will be derived first, then the waveform for the chain reaction.

\subsubsection{The $\mathrm{F}+\mathrm{H}_{2}$ Reation}

If the rate constant for reaction 4.4 is $k_{1}$, the rate of disappearance of fluorine atoms is

$$
-d|F| / d t=k_{1}[F]\left\lfloor H_{2}\right\rfloor,
$$

where the brackets designate the density of the species and the minus sign represents disappearance of $[F]$. If there is a large excess of $\mathrm{H}_{2}$ over $F$, the value of $\left[\mathrm{H}_{2}\right]$ will be roughly constant. If the initial density, $[F]$, is $F_{0}$ the solution of $\mathrm{Eq} .4 .11$ is

$$
[F]=F_{0} \quad \exp \left(-k_{1}\left[\mathrm{H}_{2}\right] t\right) \text {. }
$$

We note also that

$$
d[H F] / d t=-d[F] / d t=k_{1} F_{0}\left[H_{2}\right] \exp \left(-k_{1}\left\{H_{2}\right] t\right) .
$$

This is the rate of formation of $\mathrm{HF}$ so the pumping terms in Eq. 4.6 are proportional to this. In particular, $\mathrm{S}_{3}=.25 \mathrm{~d}[\mathrm{HF}] / \mathrm{dt}$. Equation 4.13 says that the pulse shape will be an exponential decay. Examination of experimental waveforms shows that the later part of the pulse is approximately an exponential decay. The early part of the pulse has some additional structure as shown in Fig. 3.2. There is a delay while the population inversion builds up to threshold and further delay while the intensity builds up. The $v=2$ to $v=1$ transitions lase first because $v=2$ is the 
most strongly pumped. The $v 1-$ ?) and v3-2 transitjons lase a hit later and form tho srocond spike. liron then on the pulse Jollows the exponential deray.

An HF laser can also be made to operate il a hydrocarbon or metal hydride is substituted for $\mathrm{H}_{2}$ in reaction 4.4. The value of $k_{1}$ will then be different and the pulse width will be correspondingly different. A muner of experiments tave been performed with various hydrocarbons and metal hyrlides. The pulse widths have been measured and rate constants obtained from $\mathrm{Eq} .4 .13$. Where the rate constants were known from other sources the agreement was quite gool. Details of this work are reported in Creighton (1972) and Pearson, et al. (1973b).

Equation 4.13 was obtained assuming no consumption of $\mathrm{H}_{2}$. This restriction is easily removed by noting that $\left\{H_{2}(t) \mid=H_{0}-F_{0}+\lfloor F(t)\}\right.$, where $H_{0}$ is the value of $\left[H_{2}\right\rfloor$ at $t=0$. This gives

$$
\mathrm{d}\left[\mathrm{F} \mid / \mathrm{dt}=-\mathrm{k}_{1}[\mathrm{~F}]\left\{\mathrm{H}_{0}-\mathrm{F}_{0}+[F]\right\}\right. \text {. }
$$

The solution of this is straightforward, yielding

$$
d[H F] / d t=A H_{0} F_{0} e^{-A t} /\left(H_{0}-F_{0} e^{-A t}\right)
$$

with

$$
A=k_{1}\left(H_{0}-F_{0}\right)
$$

This agrees with $\mathrm{Eq}_{\mathrm{q}} 4.13$ when $\mathrm{F}_{0} \ll \mathrm{H}_{0}$, and remains valid provided $\mathrm{F}_{2}<\mathrm{H}_{0}$. A similar solution can be obtained for the case $\mathrm{F}_{0}>\mathrm{H}_{0}$ by expressing $[F]$ in terms of $\left[\mathrm{H}_{2}\right\rfloor$ and solving for $\mathrm{d}\left[\mathrm{H}_{2}\right] / \mathrm{dt}$. The case where $\mathrm{F}_{0}=\mathrm{H}_{0}$ can also be readi1y 
solved. The solutions are shown in Fig. 4.1, where the ratio $\mathrm{NF}_{3} / \mathrm{H}_{2}$ was varied while keeping the total number density constant. Three dissociation fractions of $\mathrm{F}_{0} / \mathrm{NF}_{3}$ are shown along with the solution of Eq. 4,13.

4.2.3 The Chain Reaction of $\mathrm{H}_{2}$ with $\mathrm{F}_{2}$

The chain reaction case $G i f f e r s$ from the single reaction just discussed, in that it involves two reactions

$$
\begin{aligned}
& \mathrm{F}+\mathrm{H}_{2} \stackrel{\mathrm{k}_{1}}{\longrightarrow} \mathrm{HF}+\mathrm{H} \\
& \mathrm{H}+\mathrm{F}_{2} \stackrel{\mathrm{k}_{2}}{\longrightarrow} \mathrm{HF}+\mathrm{F}
\end{aligned}
$$

The relevant kinetic rate equations are

$$
\begin{aligned}
& \mathrm{d}[\mathrm{F}] / \mathrm{dt}=-\mathrm{k}_{1}[\mathrm{~F}]\left[\mathrm{H}_{2}\right]+\mathrm{k}_{2}[\mathrm{H}]\left[\mathrm{F}_{2}\right] \\
& \mathrm{d}[\mathrm{HF}] / \mathrm{dt}=\mathrm{k}_{1}[\mathrm{~F}]\left[\mathrm{H}_{2}\right]+\mathrm{k}_{2}[\mathrm{H}]\left[\mathrm{F}_{2}\right]
\end{aligned}
$$

and $d\left[\mathrm{H}_{2}\right] / d t=-k_{1}[\mathrm{~F}]\left[\mathrm{H}_{2}\right]$.

The reaction now is controlled by consumption of $\mathrm{H}_{2}$ or $\mathrm{F}_{2}$, whichever is smaller. (In the case of the single reaction, (Eq. 4.4) consumption of $F$ atoms was dominant.) The chain reaction rapidly reaches a steady state where the $F$ consumed by reaction 4.4 is replaced by reaction 4.7 , and both $\mathrm{H}_{2}$ and $\mathrm{F}_{2}$ are consumed at the same rate. We will first obtain the steady-state solution and then the transient.

For the sake of algebraic simplicity, we will treat the important special case where the initial values of $\left[\mathrm{H}_{2}\right]$ and $\left\{F_{2}\right]$ are equal. This case is important because it gives 
the maximum rotal energy for a lixed total pressure. Pcak power can be increased, at the cost of shorter duration and less cuergy, by making the mixture rich in $F_{2}$ to conpensitr for the fact that $k_{2}<k_{1}$.

we can obtain the strady-state solution by setting. $\mathrm{E}_{1}$. 4.16 to zero, giving

$$
\mathrm{k}_{1}[\mathrm{~F}]\left[\mathrm{H}_{2}\right]=\mathrm{k}_{2}[\mathrm{H}]\left[\mathrm{F}_{2}\right] \text {. }
$$

In this special case $\left[\mathrm{H}_{2}\right]=\left[\mathrm{F}_{2}\right]$, and in general $[\mathrm{H}]=\mathrm{F}_{0}-[\mathrm{F}]$, because we assumed no recombination to remove atoms, and there is no additonal source of atoms once $F_{0}$ atoms have been dissociated. The steady-state value of $[F]$ is $F_{0} k_{2} /\left(k_{1}+k_{2}\right)$. Putting, this in $\mathrm{Eq} .4 .18$ gives

$$
\frac{d\left[\mathrm{H}_{2}\right]}{\left[\mathrm{H}_{2}\right]}=\frac{-\mathrm{k}_{1} \mathrm{k}_{2}}{\mathrm{k}_{1}+\mathrm{k}_{2}} \quad \mathrm{~F}_{0} \mathrm{dt}
$$

which has the solution

$$
\left[\mathrm{H}_{2}\right]=\mathrm{H}_{0} \exp \left[-\mathrm{k}_{1} \mathrm{k}_{2} \mathrm{~F}_{0} \mathrm{t} /\left(\mathrm{k}_{1}+\mathrm{k}_{2}\right)\right],
$$

where $\mathrm{H}_{0}$ 's the inj.tial of densicy of $\mathrm{H}_{2}$. Inserting the expressions for $[F]$ and $\left[\mathrm{H}_{2}\right]$ in $\mathrm{Eq}_{\mathrm{q}} .4 .17$ gives the rate of formation of $\mathrm{HF}$,

$$
\mathrm{d}[H F] / \mathrm{dt}=\frac{2 \mathrm{k}_{1} \mathrm{k}_{2}}{\mathrm{k}_{1}+\mathrm{k}_{2}} \quad \mathrm{~F}_{0} \mathrm{H}_{0} \exp \left[-\mathrm{k}_{1} \mathrm{k}_{2} \mathrm{~F}_{0} \mathrm{t} /\left(\mathrm{k}_{1}+\mathrm{k}_{2}\right)\right] .
$$

This is an exponential decay as was the single-step reaction; the principal difference is the sagnitude of the time constant. For the single-step reaction the time constant was $1 / \mathrm{k}_{1}\left[\mathrm{H}_{2}\right]$, for the chain reaction it is $\left(k_{1}+k_{2} / k_{1} k_{2} F_{0}\right)$. At room temperature $k_{1}$ is about $4 k_{2}$ and normally $F_{0}$ is a few percent 
of $\mathrm{H}_{0^{*}}$. These values $\mathrm{g}$ ive the chain reaction a rime constant which is an order of magnitude larger than the time constant of the single-step reaction.

Equation 4.22 ignores an initial transient resulting from the fact the $[F]$ initially equals $F_{0}$ and $[H]$ is zero. The transient value of $[F]$ can be obtained from Eq. 4.16 if consumption of $\mathrm{h}_{2}$ is neglected.

$$
\begin{aligned}
& {[F]=\frac{F_{0}}{k_{1}+k_{2}}\left(k_{2}+k_{1} e^{-t / \tau} 1\right)} \\
& 1 / \tau_{1}=k_{1}\left[H_{2}\right]+k_{2}\left[F_{2}\right]
\end{aligned}
$$

The first term of $\mathrm{Eq}, 4.23$ is the steady-state solution and the second is the transient. The transient time constant is the same order of magnitude as the single-step time constant. When Eqs. 4.21 and 4.23 are substituted in 4.17, an approximate solution is obtained which gives both the transient and the steady state.

$$
\begin{aligned}
& \mathrm{d}[\mathrm{HE}] / \mathrm{dt}=\frac{\mathrm{k}_{1} \mathrm{k}_{2}}{\mathrm{k}_{1}+\mathrm{k}_{2}} \quad \mathrm{E}_{0} \mathrm{H}_{0} \mathrm{e}^{-t / \tau} 2\left[2+\left(\mathrm{k}_{1} / \mathrm{k}_{2}-1\right) \mathrm{e}^{-t / \tau} \underline{1]}(4.25)\right. \\
& 1 / \tau_{2}=\frac{\mathrm{k}_{1} \mathrm{k}_{2}}{\mathrm{k}_{1}+\mathrm{k}_{2}} \mathrm{~F}_{0}
\end{aligned}
$$

Figure 4.2 shows this solution $\operatorname{for}\left[\mathrm{H}_{2}\right]=\left[\mathrm{F}_{2}\right]$ and $\mathrm{k}_{1}=4 \mathrm{k}_{2}$. The figure also shows the single-step solution for comparison. In summary, solutions for both the single-step reaction and the chain reaction have been obtained. The 1aser waveforms, proportional to $\mathrm{l}[\mathrm{Hl} \cdot] / \mathrm{dt}$, both have an exponential decay, but the time constant lor the chain reaction will normaliy be an order of magnitude larger. 


\subsection{The Errects of Vibrational Deactivation}

The discussion so far has neglected vibrational deactivatioll of the vibrationally excited states. This will now be rreated by perturbation methols

Vibrational energy can be converted to translational enerpy liy reactions of the form

$$
\mathrm{HF}(v)+M \stackrel{\mathrm{k}_{3}}{\longrightarrow} \mathrm{HF}(\mathrm{v}-1)+M
$$

where $M$ can be any molecule. The reverse reaction is highly improbable because very few molecules have enough translational energy to vibrationally excite $\mathrm{HF}$. In reality, we should have a series of reactions like 4.27, one for each species with a different rate for each. However, the contribution of reaction 4.27 to the deactivation rate has the form

$$
\mathrm{k}_{3}\left[\mathrm{M}^{-1}\right][\mathrm{HF}(\mathrm{v})] \text {. }
$$

Mathematically the correct results are obtained if we let $k_{3}[M]=\sum_{i} k_{i}\left[M_{i}\right]$. The largest $k_{i}$ is obtained when $\left[M_{i}\right]$ is another $H[F$. In that case, vibrational deactivation occurs once every 50 to 100 collisions. Most other molecules require $10^{3}$ to $10^{6}$ collisions to vibrationally deactivate $\mathrm{HF}$. Vibrational deactivation of HF can also occur by near-resonant transfer of a vibrational quantum to another molecule, as in the following reaction:

$$
\mathrm{HF}(\mathrm{v})=\mathrm{H}_{2}(\mathrm{O}) \stackrel{\mathrm{K}_{4}}{=} \mathrm{HF}(\mathrm{v}-1)+\mathrm{H}_{2}(1) \text {. }
$$

This differs from reaction 4.27 because the reverse rate is comparable to the forward rate. The vibrationally excited $\mathrm{H}_{2}$ 
can store energy, removing it from early portions of the laser pulse and returning some of it later. Some of the enerzy transferred to $\mathrm{H}_{2}$ will be 10 t due to vibrational deactivation of $\mathrm{H}_{2}$. The rate for vibration-vibration exchange, $k_{4_{4}}$, is smaller than the vibrational-trans lational exchange rate, $k_{3}$. for $H F-H F$ collisions. Therefore, we introduce little error by neglecting the energy storage and simply add $\mathrm{k}_{4}\left[\mathrm{H}_{2}\right]$ to the term

$$
\sum_{i} k_{i}\left[M_{i}\right] \text {. }
$$

We will treat vibrational deactivation by perturbation methods using Eq. 4,13 to obtain the value of $\mathrm{HF}$. Integrating Eq. 4.13 gives

$$
[\mathrm{HF}]=\mathrm{F}_{0}\left[1-\exp \left(-\mathrm{k}_{1}\left[\mathrm{H}_{2}\right] \mathrm{t}\right)\right] \text {. }
$$

When we ald vibrational deactivation to Eq. 4.13

we get

$$
d[\mathrm{HF}] / \mathrm{dt}=\mathrm{k}_{1} \mathrm{~F}_{0}\left[\mathrm{H}_{2}\right]_{0} \cdot \mathrm{e}^{-\mathrm{t} / \mathrm{t}}-\mathrm{k}_{3}[\mathrm{HF}(\mathrm{v})][\mathrm{M}] .
$$

For simplicity, consider only two levels, HF(1) and $\mathrm{HF}(0)$. In the steady state $[\mathrm{HF}(1)]=[\mathrm{HF}(0)]+\Delta \mathrm{N}_{\mathrm{c}}$, and since normally $\Delta \mathrm{N}_{\mathrm{c}}<<[\mathrm{HF}(0)],[\mathrm{HF}(1)]$ is approximately equal to $[\mathrm{HF}(2)]$, and both are approximately equal to half of $[\mathrm{HF}]$, as given by Eq. 4.29. (With more states equality would hold, but the fraction would bo smaller.) Using this value, and inserting the photons as $P / f_{i w}$, we can write equations similar to 4.2 .

$\mathrm{d}[\mathrm{HF}(1)]=\mathrm{k}_{1} \mathrm{~F}_{0} \Gamma_{1} \mathrm{H}_{0} e^{-\mathrm{t} / \tau}-1 / 2 \mathrm{k}_{3}[\mathrm{M}] \mathrm{F}_{0}\left(1-\mathrm{e}^{-\mathrm{t} / \tau}\right)-P / K_{1 \omega}$ $\mathrm{d}[\mathrm{HF}(0)]=\mathrm{k}_{1} \mathrm{~F}_{0}{ }_{0} \mathrm{H}_{0} \mathrm{e}^{-t / \tau}+1 / 2 \mathrm{k}_{3}[\mathrm{M}] \mathrm{F}_{0}\left(1-\mathrm{e}^{-t / \tau}\right)+\underset{(4.31)}{\mathrm{P} / \mathrm{j} \omega}$ Where $f_{1}$ is the rraction of $\mathrm{HF}$ created in state $1, f_{0}$ is the corresponding Iraction in state 0 , and $1 / \tau=k_{1} \mathrm{H}_{0}$. The 
condition $\mathrm{d}[\mathrm{HF}(1)] / \mathrm{dt}=\mathrm{d}[\mathrm{HH}(0) / \mathrm{d} t$ gives

$p / X_{1 \omega}=1 / 2\left(f_{1}-f_{0}\right) k_{1} H_{0} F_{0} e^{-t / \tau}-1 / 2 k_{3}[M] F_{0}\left(1-e^{-t / \tau}\right)$

This is shown in Fig. 4.3 with $2 \mathrm{P} / \mathrm{H}$ w laboled "Laser," the first trrm of the right labeled "[Hil"]" and the second labeled "Vib. Deact." The figure clearly shows that vibrational deactivation reduces both the pulse width and the total energy. However, the effect is fairly small because $k_{1}=10 k_{3}$.

A similar result can be obtained for the chain reaction, neglecting the transient term in $\mathrm{Eq} .4 .24$ and noting that in this case $[H F]=H_{0}\left(1-e^{-t / \tau} 2\right)$. The result is

$$
\begin{aligned}
2 \mathrm{P} / \mathrm{h \omega} \omega & =1 / 2\left(\mathrm{f}_{1}-\mathrm{f}_{0}\right) \frac{\mathrm{k}_{1} \mathrm{k}_{2}}{\mathrm{k}_{1}+\mathrm{k}_{2}} \mathrm{~F}_{0} \mathrm{H}_{0} \mathrm{e}^{-\mathrm{t} / \tau_{2}} \\
& -1 / 2 \mathrm{k}_{3}[\mathrm{M}] \mathrm{H}_{0}\left(1-\mathrm{e}^{-\mathrm{t} / \tau 2}\right) .
\end{aligned}
$$

This is similar to Eq. 4.32 exeept that $\mathrm{k}_{3}$ [M] may be comparable to $k_{1} k_{2} F_{0} /\left(k_{1}+k_{2}\right)$, because $F_{0}<<[M]$. In the case of the chain reaction the laser pulse may be terminated by vibrational deactivation, instead of consumption of $\mathrm{H}_{2}$. Thus vibrational deactivation may be a major factor in the total laser energy in the case of a chain reaction. 4.4 Variation of Laser Pulse Energy with Pressure The experimental variation of total laser energy wj.th pressure is shown in Fig. 3.5. There is clearly a tnaximum at 60 to 80 torr and a decrease in total pulse energy at higher pressures. Additional data shows that the peak power also reaches a maximum at about 80 torr and then drops off. (Pearson, et al., 1973a) The total energy drops off 
faster because the pulse width is also decreasing with increasing pressure. We will compare our analytic model with these results and find that the model predicts a monotone increasing output energy as the pressure increases, provided the fraction of $\mathrm{NF}_{3}$ dissociated to form $\mathrm{F}$ atoms is the same at a11 pressures. This is true when we include any other two-body collisional deactivation process. We will examine three-body process and find that they predict a decrease in output energy at higher pressures. However, when numerical values are inserted in the equations the peak energy occurs at pressures which are orders of magnitude larger than the experimental pressure. It therefore appears that at higher pressures a smaller fraction of $\mathrm{NF}_{3}$ is dissociated.

\subsubsection{Two-Body Reactions}

The laser power when vibrational deactivation is neglected is proportional to $\mathrm{d}[\mathrm{HF}] / \mathrm{dt}$ as given by $\mathrm{Eq} \cdot 4.13$ (or Eq. 4.15). This equation shows monotone increasing peak power with increasing pressure. Also equation 4.13 is easily integrated to show that the total pulse energy is proportional to $F_{0}$.

When two-body vibrational deactivation is added, the output power is given by Eq. 4.32. Again the power increases with increasing pressure. To obtain the total pulse energy we must integrate $\mathrm{Eq} \cdot 4.32$. The integration must be sut off when the power decays to zero. This occurs at a time

$$
T=\tau \ln \left(\frac{f_{1}-f_{0}+\mu_{3}}{u_{3}}\right)
$$

where $\quad 1 / \tau=k_{1} H_{0}$ and $\mu_{3}=\left(k_{3}\left[M^{-}\right]\right) /\left(k_{1} H_{0}\right)$ 
When $t>T$ the steady state broaks down, Eq. 4.32 is no 10 geer valid, and clearly there is no ndditional power oulput. Integrating Eq. 4.32 from 0 to $T$ gives the total pulse energy

$$
W_{\text {Tota } 1}=1 / 2 \chi_{\omega} F_{0}\left\{\mathrm{r}_{1}-\mathrm{I}_{0}-\ln \left[1+\left(\mathrm{f}_{1}-\mathrm{f}_{0}\right) / \mathrm{H}_{3}\right] / \mathrm{H}_{3}\right\}(4.35)
$$

The quantity in urackets is dimensionless, and independent of pressure. Therefore vibrational deactivation does not cause lower output energy at high pressures. It can te shown that this is true for any process that depends on two-body collisions. 4.4.2 Three-Body Reactions Recombination Some chemical reactions involve three bodies and these reactions contribute terms of the form $k[A][B][C]$ to the rate. Consider, for example, the reaction

$$
\mathrm{F}+\mathrm{F}+\mathrm{M} \stackrel{\mathrm{k}_{5}}{\longrightarrow} \mathrm{F}_{2}+\mathrm{M}
$$

Where $M$ is some molecule. If the significant chemical reactions are 4.4 and 4.9 , then

$$
\mathrm{d}[\mathrm{F}] / \mathrm{dt}=\mathrm{k}_{1}[\mathrm{~F}]\left[\mathrm{H}_{2}\right]-\mathrm{k}_{5}[\mathrm{~F}][\mathrm{F}][\mathrm{M}] \text {. }
$$

This equation has the solution

$$
[F]=F_{0} e^{-t / \tau}\left[\frac{k_{1} H_{0}}{k_{1} H_{C}+k_{5} F_{0}[M]\left(1-e^{-t / \tau}\right)}\right]
$$

and the laser power is proportional to the rate of creation of $\mathrm{HF}$,

$$
\mathrm{d}[\mathrm{HF}] / \mathrm{dt}=\mathrm{k}_{1}\left[\mathrm{H}_{2}\right][\mathrm{F}] .
$$

In the event the $k_{5}$ is zero, substituting (4.37) in (4.38) gives $\mathrm{Eq} .4 .13$, as it should. In the event the $k_{5}$ is reasonably large, the power will drop off much faster, although the initial peak will be the same size. This will occur when 
$\mathrm{k}_{1} \mathrm{H}_{0} / \mathrm{k}_{5}[\mathrm{M}]\left[\mathrm{F}_{0}\right]$ is on the order of unity. At $300 \mathrm{~K}, \mathrm{k}_{1}$ is about $10^{12} \mathrm{~cm}^{3} \mathrm{msie}^{-1} \mathrm{sec}^{-1}$ (Cohen, 1971) and $k_{5}$ is less than $10^{16} \mathrm{crit}^{\epsilon}$ mole $\mathrm{sec}^{-1}$. With $F_{0}$ approximately $0.1 \mathrm{H}_{0}$ the value of $[\mathrm{M}]$ would have to be about $10^{-3}$ moles $/ \mathrm{cm}^{3}$ for the two terms to be equal in magnitude. This corresponds to a pressure of 20 atmospheres, but experimentally the peak occurs at about 0.1 atmosphere. There is some uncertainty in the exact value of $k_{5}$ (Cohen, 1971), but it could scarcely be that large. Also Eqs. 4.37 and 4.38 show that the threebody reconbination reduces the pulse width at, say, $10 \%$ of peak. If this is plotted versus pressure, the line will curve at high pressure. This is not seen in experimental data.

It appears, therefore, that three-body recombination of $F$ cannot account for the drop in laser power at higher pressures. The same argument applies to reaction 4.10 , especially since it creates HF. Reaction $4.8 \mathrm{might}$ actually enhance the power because it restores some of the $\mathrm{H}_{2}$ which was considered to be consumed in the previous analysis. Thus we conclude that recombination reactions 4.8 to 4.10 cannot account for decrease in laser energy and power at pressures above 80 torr.

\subsubsection{Three-Body Vibrationa1 Deactivation \\ W. Klemperer (1972) has suggested that the} anomalous ly high vibrational deactivation rate of $\mathrm{HF}$ may be due to dimer formation. It is well known that dimers, trimers and hexamers of HF exist in equijibrium with the monomer at room temperature (Kollman and Allen, 1970, Del Bene and Pople, 1971, Franck and Meyer, 1959; Dyke, Howard and Klemperer, 1972; 
Smith, 1958 and 1959). The proposed mechanism for deactivation is that two HF molecules collide and that before they can separate again the complex is struck by a third molecule. This third molecule absorbs vibrational energy which causes the two HF molecules to be bound into a dimer but in a high vibrational state. The bondinf is known to be a hydrogen bond with a bond energy of about $6 \mathrm{kcal} / \mathrm{mole}$. Once the bond is formed the vibrational modes can exchange energy. This may lead to unimolecular decomposition, where after the dimer has suffered up to 10,000 collisions with other molecules it: will break apart, but with a high probability that the resulting $\mathrm{HF}$ molecules have lost their vibrational energy. The net result is an apparent deactivation of the vibrationally excited $\mathrm{HF}$ or even a temporary apparent removal of $\mathrm{HF}$ molecules from the system. We wish to estimate the magnitude of this effect.

The three-body reaction can be written

$$
\mathrm{HF}(\mathrm{V})+\mathrm{HF}+\mathrm{M} \stackrel{\mathrm{k}_{6}}{=} \mathrm{H}_{2} \mathrm{~F}_{2}+\mathrm{M}_{1}
$$

where we assume that at least one of the HF molecules is vibrationally excited. Klemperer (1972) has suggested that the rate constant, $\mathrm{k}_{6}$, should be taken to be the three-body gas kinetic collision frequency. This was estimated by assuming that two HF molecules come together in a binary collision and form a complex with a lifetime of roughly one vibrational period. The density of these short-lived complexes 
with other molecules can be computed. If the binary collision cross section is $10^{-15} \mathrm{~cm}^{2}$ and the vibrational period is $10^{-13}$ seconds, then $k_{6}$ is approximately $5 \times 10^{17} \mathrm{~cm}^{6} \mathrm{~mole}^{-2} \mathrm{sec}^{-1}$.

To obtain the laser power we neglect recombination and consumption of $\mathrm{H}_{2}$ so that the value of $[\mathrm{F}]$ is given by Eq. 4.11. If we consider only two vibrational levels the laser power is

$P / h_{\omega}=1 / 2\left(f_{1}-f_{0}\right) k_{1} H_{0} F_{0} e^{-t / \tau}-1 / 2 k_{6}[M][H F]^{2} \cdot$ (4.40) In the second term the $1 / 2$ comes from the lact that approximately one half of the HF is in the upper vibrational state. Substituting the value of [HF] from Eq. 4.29 gives $P / h_{\omega}=1 / 2\left(f_{1}-f_{0}\right) k_{1} H_{0} F_{0} e^{-t / \tau}-1 / 2 k_{6}[M] F_{0}{ }^{2}\left(1-e^{-t / \tau}\right)^{2}$.

This is similar to Eq. 4.32 except that the second term now depends on the third power of the pressure, assuming $F_{0}$ proportional to the pressure. The total pulse energy is obtained by integrating Eq. 4.41 to the time, $T$, where $P$ goes to zero. For this purpose we re-write Eq. 4.41

$$
P=A p^{2} e^{-t / \tau}-B p^{3}\left(1-e^{-t / \tau}\right)^{2}
$$

where $p$ represents the pressure. We can find the time when the power goes to zero if we neglect $e^{-2 T / \tau}$ compared to $e^{-T / \tau}$

$$
T=\tau \ln \left(\frac{A+2 B p}{B_{p}}\right)
$$

The total pulse energy is then given approximately by 
$W=A p^{2} r\left[\frac{A+B p}{A+2 B p}\right]-B p^{3} \tau\left[T / \tau+2\left(\frac{A+B p}{A+2 B p}\right)+1 / 2-1 / 2\left(\frac{B p}{A+2 \bar{B}}\right)^{2}\right]$ (4.43)

The terms in square brackets vary slowly with pressure; and since $\tau \propto \mathrm{p}^{-1}$, the rirst torm is rouphly linear in pressure and the second quadratic. As a result, Eq. 4.43 approximates a parabola wi.th a maximum when $\mathrm{Ap}^{2}=\mathrm{Bp}^{3}$, or when $\left(f_{1}-i_{0}\right) k_{1} H_{0} F_{0}=k_{6}[M] F_{0}^{2}$. To evaluate this numerically, assume the $\left(\mathrm{f}_{1}-\mathrm{r}_{0}\right)=1 / 3$ and that $[\mathrm{M}]=\left[\mathrm{H}_{2}\right]+\left[\mathrm{NF}_{3}\right] \approx 6 \mathrm{H}_{0}$. The measured value of $\mathrm{k}_{1}$ is $2 \times 10^{13} \mathrm{~cm}^{3}-\mathrm{mole}^{-1} \mathrm{sec}^{-1}$ and $k_{6}$ is estimated above as $5 \times 10^{17} \mathrm{~cm}^{6} \mathrm{~mole}^{-2} \mathrm{sec}^{-1}$. The equality is then satisfied when $F_{0}=2.2 \times 10^{-6}$ moles $/ c c$, equivalent to a pressure of about 40 torr. Since $F_{0}$ seems to be less than a tenth of the total initial pressure, this would require a total pressure around 400 torr, five times higher than the experimentally observed maximun. Thus HF dimer formation doesn't seem to account for the fall-off of laser pulse energy above 60 torr. Not only is the magnitude of the elfect too sma11, but Eq. 4.41 shows that the peak laser power should continue to increase with pressure, in contrast to experimental results.

Three possible reasons for the fall-off in laser energy and power have been investigated, three-body recombination of fluorine atoms, vibrational deactivation of $\mathrm{HF}$ by three-body reaction which form dimers, and a peak in the discharge energy (See Section 3). All three mechanisms give approximately the correct shape for the laser energy 
versus pressure curve, but the calculated pressure for maximum energy is one to two order of magnitude too high. 1t appears that the reason for the fall-cff in energy is likely to be found in the physics of the discharge, but that is not we11 understood at present. 


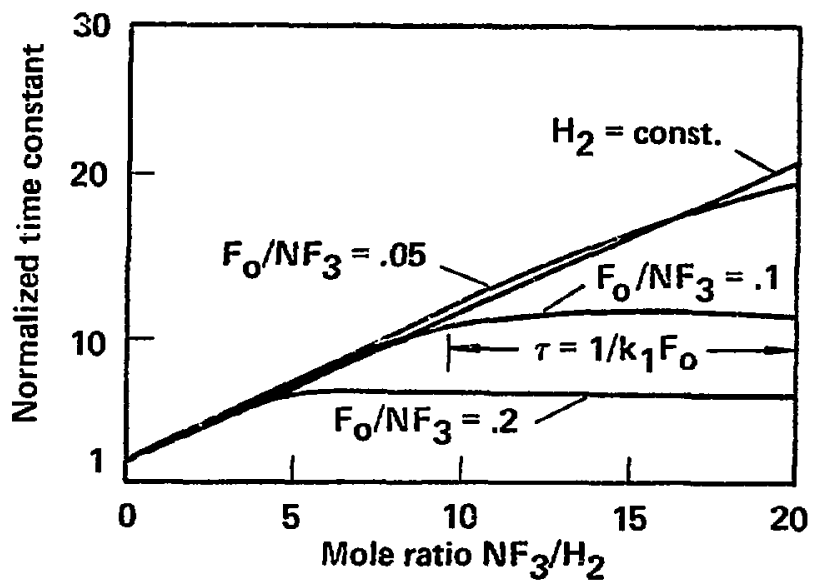

Fig. 4.1 Varlation of laser pulsewidth as a function of the ratio $\mathrm{NF}_{3}: \mathrm{H}_{2}$ at constant total pressure. Curves are shown for three initial dissociation ratios and for the case with $\mathrm{H}_{2}$ assumed constant. In the lower right hand corner the pulse is limited by consumption of $\mathrm{H}_{2}$. 


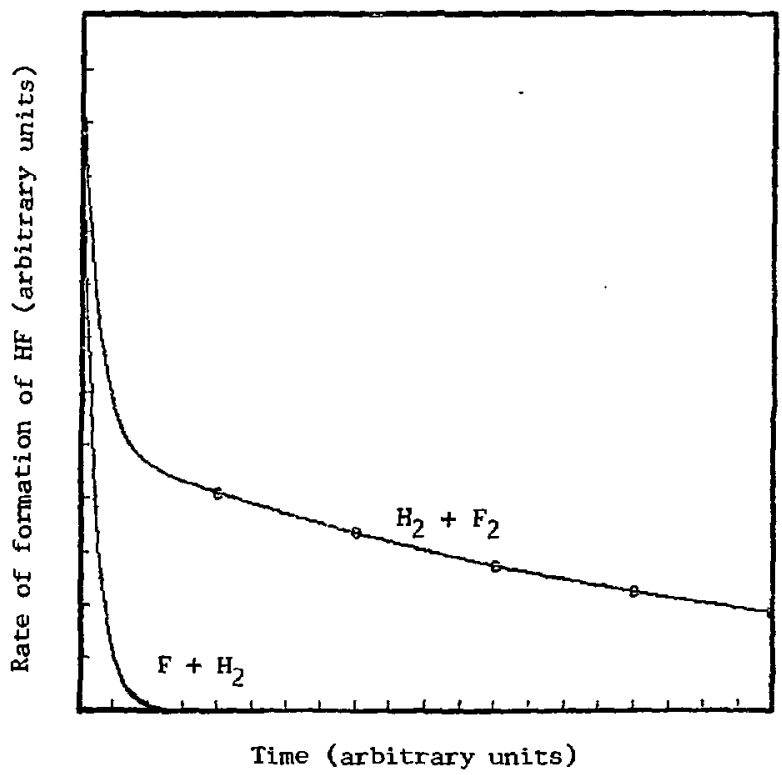

Fig. 4.2 Comparison of rates of formation for chain reaction $\left(\mathrm{H}_{2}+\mathrm{F}_{2}\right)$ and $\mathrm{F}+\mathrm{H}_{2}$. Initial conditions: $\left[\mathrm{H}_{2}\right]=\left[\mathrm{F}_{2}\right]=10[\mathrm{~F}]$, and $\mathrm{k}_{1}=4 \mathrm{k}_{2}$. 


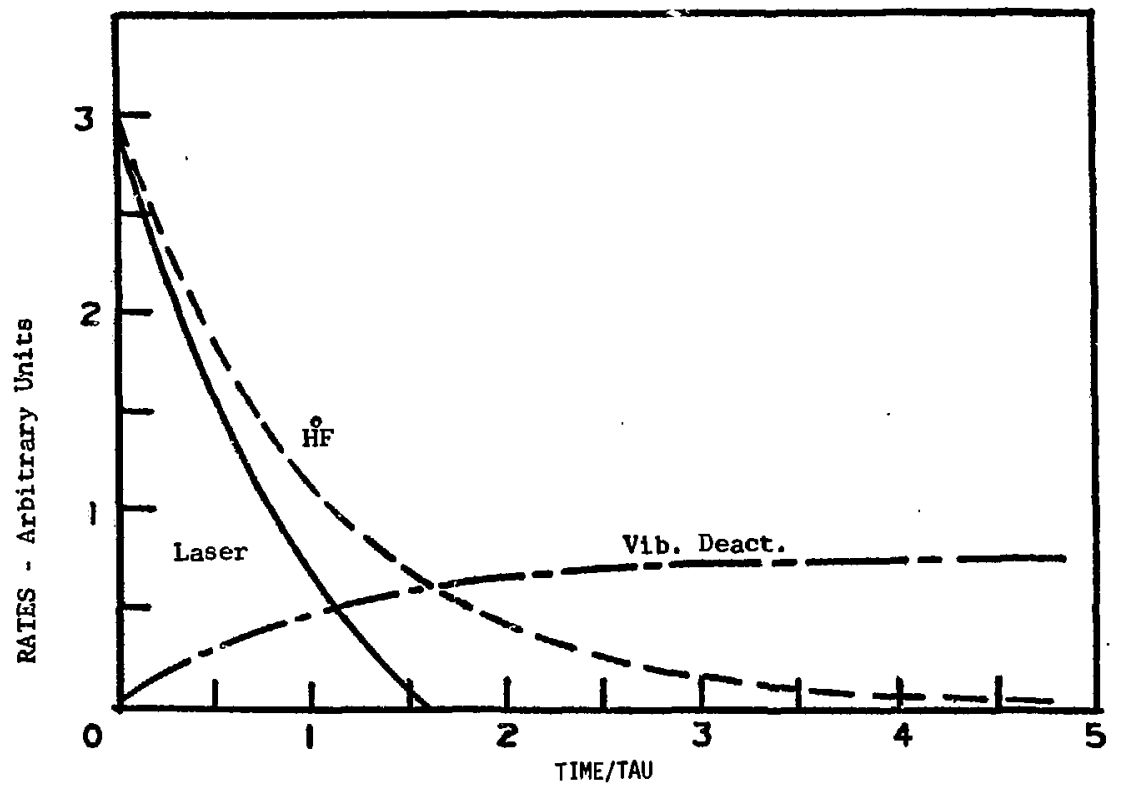

Fig. 4.3 Effect of two body vibrational deactivation on laser output. Laser output is HiF minus Vib. deact. 
5. THE MINOTAR CODE FOR CALCULATING CHEMICAL KINETICS

5.1 Introduction

The analytic model of the previous section is based on approximations. Equations 4.13 and 4.22 were derived neglecting vibrational de-activation, recombination, and consumption of some reactants. These effects were later included by means of approximations and perturbation techniques. Exact solutions require numerical techniques. There are two reasons for this. The more fundamental reason is that the equations are non-linear in the dependent variables. Specifically, a term such as $\mathrm{k}_{1}[\mathrm{~F}]\left[\mathrm{H}_{2}\right]$ involves the product of two dependent variables, the densities of flourine atoms and hydrogen molecules. Differential equations containing such terms can only be solved analytically under rather special conditions. In order to solve more general cases numerical solutions are required. The second reason for using numerical solutions is that a large number of variables are involved and the computer is more or less required just to do the necessary bookkeeping. For example, in the chain reaction, $\mathrm{H}_{2}+\mathrm{F}_{2}$, seven vibrational levels of $\mathrm{HF}$ are involved. If we include formation and vibrational deactivation of $\mathrm{HF}$ along with radical formation and recombination and chain branching, mechanisms we get 60 to 100 chemical reaction steps, and perhaps fifteen species as dependent variables. Even if this could be solved analytically, the labor involved in keeping track of these variables would be enormous. 
for these reasons the MINOTAR code? was constructed. The analytic approximations are guite useful for obtaining physical insight, but the nunerical solutions are useful for cstablishing the validity of the approximations. (Jn fact, the approximations leading to Eq. 4.13 were first seen in the process of sctting up a test problen to verify the MINOTAR (cole.)

5.2 The Gear Method for Solving Differential Equations The usual methods for solving ordinary differential cquations numerically are Runge-Kutta, the Adams predictorcorrector and jts variants, Nordsieck's method, and Hamming's method, a particularly useful form of predictor-corrector method. The Runge-Kutta method is frequently used because it is quite easy to change the step size of the independent variable.

The initial attempt to solve the chemical kinetics problem used a Runge-Kutta method, but it immediately became clear that it would take tens of minutes of computer tince to solve a single problem, lurther investigation revealed that the chemical kinetios equations contained several time constants differing by several orders of magnitude. Such equations are known as "stiff" equations, apparently because they were first recognized in structural analysis. All of the standard techniques for solving ordinary differential equatious have a domain of stability which limits the time step to a small multiple of the shortest time constant (Seinfeld, et al., 1970). If a larger time step is chosen, errors due 
to machine roundof $f$ and truncation errors in the numerical procedure are amplified at each time step and the solution rapidly goes to infinity. The situation is analogous to using the fixst few terms of a slowly converging infinite series, for example a cosine. Unless the argument is kept small, the first few terms of the series give an erroneous resilt. The chemical reactions which cause problems are reactions such as radical formation or recombination, which are essentially at equilibrium over most of the reaction, but can change very rapidiy. Minor errors in these reactions can be amplified if the time step becomes too large. When these reactions are actually creating or removing radicals we want to follow them. When they are in equilibrium we don't want them controling the time step.

One way to achieve this is to add Iogic which checks to see if these equations are near equilibrium and to solve them algebraically in that case. This has been done in the Aerospace Corporation's NEST code (Emanue1, 1964; Turner, Adams and Emanuel, 1973), and works satisfactorily. The logic, however, is fairly complicated. One is also concerned that forcing the equations to steady state may introduce errors.

Another method, perhaps more elegant, is to use Gear's method to solve the stiff system of equations (Gear, 1971a, $1971 \mathrm{~b}$; Hindmarsh, 1972a, 1972b, 1973). Gear's method is a predictor-corrector which is arranged to be stable against noise in the terms with fast time constants. The method 
uses numerical approximations to high-order derivatives for the prodictor and corrector stejs. Tho values of the dependent variables are obtained from a truncated series of the form

$$
y(t+\Delta t)=y(t)+a_{1} h y^{1}+\ldots \ldots a_{n} h_{n} y^{(n)}
$$

where $h$ is the time step and $y(n)$ is the nth clerivative of $y$. The coefficients $a_{1} \ldots a_{n}$ are chosen to make the truncated series give bounded error for those terms which have fast time constants but are near equilibrium. The order of the truncated series is the number of derivatives. The order may vary from 1 to 6, with a different set of coefficients, $a_{n}$, for each order. The derivatives are stored in the form $h^{n} y^{(n)}$, so that the time step, $h$, is easily changed by simply rescaling the derivatives. Tests and controls for changing the time step and order are included in Gear's algorithm (Gear, 1971a, Hindmarsh, 1972a). The power of this method is best shown by an example. The chain reaction, $\mathrm{H}_{2}+\mathrm{Br}_{2}$, was run to $t=10^{13}$ seconds in less than three hundred time steps. The first step was only $10^{-10}$ seconds, but the last step was $10^{12}$ seconds. The normal Runge-Kutta method would have kept the time step at less than $10^{-6}$ seconds for this problem. The Gear method has a disadvantage for some problems. In order to get large time steps the corrector used a generalized Newton-Raphson iteration technique. This requires inversion of the Jacobian matrix $\left|\partial \dot{y}_{i} / \partial y_{k}\right|$, where $\dot{y}_{i}$ is $d y_{i} / d t$. In problems with a large number of variables, evaluation and inversion of this matrix requires considerable computation. If the problen is not stiff, it may be more 
efficient to use another mothod. It turns out that by proper choice of the coefficients, $a_{n}$, the Gear method is converted to an Adams predictor-corrector. Gear has included a flag in his algorit' ${ }^{2} \mathrm{~m}$ which allows the user to select the Adams method up to order 12. This can be useful for some problems. The chemical kinetics problems considered so far have had only 10 or 15 variables, so the stiff method has been chosen.

\subsection{Details of the Code}

5.3.1 Computing the Derivatives

Regardless of the method of integration, the chemical

reactions must be put in a form that can be used by the computer. If $Y_{i}$ is the density of chemical species $i$, the reaction numbered $j$ can be written

$$
A_{I j} Y_{I}+A_{K j} Y_{K} \rightarrow B_{L j} Y_{L}+B_{M j} Y_{M}
$$

where $A_{i j}$ and $B_{i j}$ are stoichometric mubers for species $i$ in reaction $j$. When $A$ and $B$ are stored as matrices the rate equation for species $Y_{k}$ can be written (Emanuel, 1971)

$$
\begin{aligned}
\mathrm{dY}_{k} / \mathrm{dt}=\sum_{j}\left\{-\mathrm{A}_{\mathrm{k} j} \mathrm{k}_{j} \prod_{i} \mathrm{Y}_{i}{ }^{\mathrm{A}_{i j}}\right. \\
+\mathrm{B}_{k j} \mathrm{k}_{-j} \prod_{i} \mathrm{Y}_{i}{ }^{\left.\mathrm{B}_{i j}\right\}}
\end{aligned}
$$

The forward reaction rate is $k_{j}$ and the reverse rate is $k_{-j}$. The forward reaction depletes the density of $Y_{k}$ (if $A_{k} \neq 0$ ); and the reverse reaction increases the density if $B_{k} \neq 0$. The forward and reverse rates are related by the equilibrium constant, $k_{p}=k_{j} / k_{-j}$. This in turn is obtained from 


$$
K_{p}=\exp \left(-\Delta G_{f}^{*} / R^{*}\right),
$$

wherc $\Delta G_{f}^{*}$ is the standard Gibbs free energy of formation at the temperature $T$. Since both the rates and $\Delta G_{f}^{*}$ are functions of temperature, the code must also be able to calculate the temperature.

In the MINOTAR code the densities of the species $Y_{j}$ are stored as a vector and the stojchiometric numbers $A_{i j}$ and $B_{i j}$ as matrices. A subroutine named DIFFUN furnishes a vector $\mathrm{dY}_{\mathbf{i}} / \mathrm{dt}$ to the Gear subroutine. The algorithm is based on Eq. 5.3 and a listing of DIFFUN is given in Appendix $E_{\text {. T The rates }} k_{j}$ and $k_{-j}$ are stored as vectors and are computed by a thermodynamic subroutine named THERMO.

5.3.2 Computing the Thermodynamics

The function of subroutine THERMO is to calculate the temperature and then the forward and backward rates. It can do this for either an isothermal reaction or a constant volume adiabatic reaction, assuming ideal gases. (Corrections for heat transfer could easily be added by means of an additional subroutine.) In order to calculate the temperature for an adiabatic reaction THERMO must store the enthalpy of formation at $298 \mathrm{~K}$ and coefficients to calculate the specific heat (or enthalpy) for each species as a function of temperature. The forward rates are assumed to be in the form

$$
k_{j}=A_{j} T^{B} j \exp \left(-E_{a j} / R T\right) \text {, }
$$

and are stored as $A_{j}, B_{j}, E_{a j}$, for each reaction. To obtain 
reverse rates, coefficients to compute $\Delta G_{f}^{*}$ for each species nust also be stored. Subroutine THERMO first calculates the temperature and then for each reaction it calculates the forward rate, the equilibrium constant and the reverse rate. The temperature calculation requires some comment. The state of the system can be described in terms of the temperature and the densities, since we assirne constant volume and an ideal gas law. Let the densities be represented by a single variable, $\rho$. The input routines specify initial temperature $T_{0}$ and density $\rho_{0}$. At time step $k$ the Gear subroutine gives the density $\rho_{k}$. The schematic thermodynamic path for the temperature calculation is shown in Fig. 5.1. Point $i$ represents the initial state $\left(\mathrm{T}_{0},{ }_{0}\right)$. Point 1 represents the state $\left(T=298 \mathrm{~K}, \rho_{0}\right)$ and point 2 represents $\left(298, \rho_{k}\right)$. The energy at point 2 is defined as the heat of formation at $298 \mathrm{~K}$ for densities $\rho_{k}$, i.e..

$$
E_{2} \equiv \Delta H_{f}^{298}\left(o_{k}\right)-o_{k} R T_{2} \text {. }
$$

Similarly, at point 1

$$
E_{l} \equiv \Delta H_{f} 298\left(\rho_{0}\right)-o_{0} R_{1}
$$

and at point $i$

$$
E_{i}=E_{1}+\int_{298}^{T_{0}} c_{v}\left(\rho_{0}\right) d T .
$$

On the first pass through THFRMO, $E_{i}$ is calculated and stored. On succeeding stcps the Gear subroutine gives $\rho_{k}$ and we calculate $E=E_{2}-E_{i}$. Since the system is assumed to be adiabatic the energy at state $k$ is the same as that at state $i$, so that going from point 2 to point $k$ at constant density 
$o_{k}$ we have

$$
\int_{298}^{T_{k}} C_{v}\left(\rho_{k}\right) d T=\Delta E .
$$

The integral has to bo calculated iteratively because the final temperature, $T_{k}$, is unknown. For each species, $Y_{i}$, coefficients for the specific heat, $a_{k i}$, are stored so that

$$
\begin{aligned}
c_{v}(T) & =\sum_{i} Y_{i}\left(a_{1 i}-R\right)+\sum_{i} Y_{i} a_{2 i} T \\
& +\sum_{i} Y_{i} a_{3 i} T^{-1} \ldots \sum_{i} Y_{i} a_{7 i} T^{-3}
\end{aligned}
$$

Each term in Eq. 5.6 can be integrated so that the integra1 of Eq. 5.5 becomes an algebraic expression in the temperature, T. Eq. 5.5 is then solved by Newton-Raphson iteration for $\mathbf{T}_{\mathbf{k}}$. The temperature at the previous time step, $T_{k-1}$; is used as the starting value and convergence to eight significant figures is achieved in one or two iterattons:

There may be more elegant ways to compute the temperature but this one has worked well, provided the densities are used properly. The Gear method and the densities for the current predictor or corrector step must be used. An attempt to calculate an interpolated temperature at the midpoint between two steps led to disastrous instabilities. The present version works well, and since it always calculates the temperature starting at the same reference state it doesn't develop cumilative errors in the temperature.

The forward and reverse reaction rates are calculated i.n a straightforward manner. A listing of subroutine THERMO can be found in Appendix E. 


\subsubsection{Input/Output Routines}

The remainder of the subroutines in the MINOTAR code handle input/output and control the Gear subroutine.

Output Format

The output consists of both tabular and graphical output. The graphical output shows pressure, total density and temperature as a function of time. In addition, the density of selected species can be displayed and the net rates of selected reactions. The input routines. allow the user to select the molecular densities to be displayed and to select which ones appear on the same plot. Similar selection is provided for graphical display of rates. The net rate displayed involves densities of molecules as well as forward and reverse rate constants. For example, if reaction $\mathrm{J}$ is

$$
A+B \stackrel{k_{5}}{=} C+D
$$

the rate which is displayed is $R(J)=-k_{r}[A][B]+k_{r}[C][D]$. Clearly

$$
\begin{aligned}
\mathrm{d}[\mathrm{A}] / \mathrm{dt} & =\mathrm{d}[\mathrm{B}] / \mathrm{dt}=\mathrm{R}(\mathrm{J}) \\
& =-\mathrm{d}[\mathrm{C}] / \mathrm{dt}=-\mathrm{d}[\mathrm{D}] / \mathrm{dt} .
\end{aligned}
$$

On the other hand, in the reaction

$$
\begin{aligned}
& 2 A+M \underset{k_{r}}{k_{t}} B+M \\
& R(J)=-k_{r}[A]^{2}[M]+k_{r}[B][M] .
\end{aligned}
$$

and

$$
d[A] / d t=2 R(J), d[B] / d t=-R(J) \text { and } d[M] / d t=0 .
$$


'how alssolute value of $R(J)$ is lisplayed on a logarithmic scale with net forward rates $(R(J)<0)$ shown as dotted lines and net reverse rates are solid linos.

The tabular output gives the same data as the graphs, arranged in a column at each time/step. Each page contains ejght columns and, in addition, all densjties, net rates and equilibrium constants at the time the page is completed. A sample page is shown in Table 5.1. Examination of the table will show that the time for printout is nearly always a "nice" number in the sense that it corresponds to a multiple of some convenient interval. This is achieved by interpolation because a time step of the code generally doesn't correspond to a convenient interval. The interval is selected by the user as an input option. This feature is a great help in comparing two separate calculations because data can be compared at iuentical times.

Under some conditions the code will take many time steps between printout intervals. In this case the code will print a column every $M$ time steps, independent of the printout interval. The time of these printouts is not a "nice" number, but the printout is included on the assumption that many time steps imply significant changes in some variable. The number of steps, $M$, can be set by the user.

Examples of the graphical output will be presented shortly. 


\section{Input Format}

The input to the MINOTAR code is in the free-format, blank-delimited form; i.e., data may be punched in any column and symbols are separated by blanks. A sample input deck is shown in Table 5.2. The free-format is convenient and greatly reduces input errors. It also allows the chemical equations to be written in readable form. The input routine recognizes certain key words such as TITLE, EQUATIONS, and PLOT. The routine then takes the appropriate branch in order to handle the data which follows. The input deck has a certain syntax which makes it essentially a simpie computer language. A user's manual for the code is available, but a brief description of the input deck will be given.

The input routine is based on free-format input subroutines written by Alex Cecil (1965). The subroutines recognize three types of symbols: numbers, alphanumeric symbols beginning with an alphabetic character, and special characters which include all punctuation marks except the period. (A symbol is a string of characters separated from other symbols by blanks.) Cecil's subroutines read the input cards and give the symbol (or its value in the case of a number) and identify the type of symbol. When an alphanumeris symbol is read, the first three letters are compared with a symbol table. The symbol tabje concains two levels of symbols: block headers and identifiers. When the symbol read in matches a block header, the input routine branches to an appropriate block of coding, where it remains until a:other 
block header is found. Within a block the routine searches for appropriate identifiers and stores the dat a which follows. The data is assumed to be in the proper sequence. The input rouline will catch many, but not 111 , errors in the sequence in which outa is entered.

The proper sequence for the input deck is the TITLE block followed by the CONTROLS block to specify the temperature (a second conTROLS block can be used to specify other control parameters). Next is the SPECIES block which jdentifies the molecular species and their initial densities. A table of molecular symbols is set up and the thermodynamic data for each species is looked up in a disc file named JANAFB. The next block, ERUATION, reads the chemical equations and sets up the reaction matrices, $A_{i j}$ and $B_{i j}$ of Eq. 5.3. The RATES block specifies the rates in Arrhenius lorm, and the PLOT block specifies the graphs to be plotted. The key work, Go, indicates the end of the input deck and tells the code to begin calculating.

Before calculation actually begins the code gives several pages of output which indicate how the routine interpreted the input data. Examination of these pages can be used to detect misinterpretation of the input data. An independently useful feature is the printout in symbolic form of the rate equations for each species, as shown in Table 5.3. This is useful because it is easy to make mistakes in obtaining these equations by hand if the set of reactions is large. 
Surprisingly, it is quite easy to write the input routine in a free-format version. An earlier version of MINOTAR used a rixed format input deck, and it took more than a week to write and debug the input routine. The free-format version was written and debugged in less than a week. This effort has been repaid several times by the time saved in setting up input decks.

5.4 Calculations of HF Kinetics 5.4.1 Simplified Reaction Scheme

The sample input (Table 5.2) and output (Tables 5.1 and 5.3) were from a calculation of the $\mathrm{H}_{2}+\mathrm{F}_{2}$ kiratics. The species labeled HF.O represents the ground state of $\mathrm{HF}$ and HF.1 represents the $\mathrm{v}=1$ state of $\mathrm{HF}$. This is a greatly simplified reaction schem because it assumes only $v=0$ and $v=1$ vibrational leve1s. It also assumes in reactions 1 and 2 that all of the $\mathrm{HF}$ is formed in $\mathrm{v}=1$. Nevertheless, this simple scheme iltustrates the essential features of the laser, as did a two-level a: lytic model.

The scheme used assumes a constant volume, adiabatic explosion beginning with 2.5 torr each of $\mathrm{H}_{2}$ and $\mathrm{F}_{2}$ in 10 torr of helium at 300 Kelvin. To start the reaction the initial partia1 pressure of $F$ atoms was set at 0.1625 torr. This could be achieved by a fast electric discharge; a flashlamp pulse would probably last a microsecond or two, which is a significant lraction of the laser pulse. Reactions 1 and 2 constitute the chain reaction. Reactions 3 and 4 represent vibrational deactivation of $\mathrm{HF}$. Reaction 3 represents the 
anomalous $1 y$ fast deactivation by $F$ and $r F$. The variation of the reaction rate with temperature doesn't follow the standard Arrhenius form (Cohen, 1971; Bott and Cohen, 1971; Shin, 1972). The negative activation energy represents a curve fit.ting and has ne physical significance. Reaction 4 is the vibrational rate for the other species in the problem. This rate is much lower than reaction 3 . Reaction 5 represents recombination of $F$. Because the back reaction is included it can also represent therial dissociation of $k_{2}$. A simslar reaction for recombination of $\mathrm{H}$ could have been included, but recombination doesn't seem to have a major affect on the lasing.

5.4.2 Simulation of the Single-Step Reaction

A subset of this schene can be used to simulate the $\mathrm{NF}_{3}+\mathrm{H}_{2}$ or $\mathrm{SF}_{6}+\mathrm{H}_{2}$ laser. In these lasers the electric discharge dissociates $\mathrm{NF}_{3}$ in about. $70 \mathrm{nsec}$ to give a ratio of densities $[\mathrm{F}] /\left[\mathrm{NF}_{3}\right]=0.0125$. A typical experiment used 12.5 torr of $\mathrm{NE}_{3}$ mixed with 2.5 torr of $\mathrm{H}_{2}$. The reaction of $\mathrm{H}$ with $\mathrm{NF}_{3}$ i.s orders of magnitude slower than reaction 2, so only reaction 1 is important in producing $\mathrm{HF}$. Because helium was already in the thermodynamic table and $\mathrm{NF}_{3}$ wasn't, the reaction was simulated by using the scheme of Table 5.2 with the rate constant of reaction 2 arbitrarily reduced four orders of magnitude. (The exact value wouldn't affect the results.) The 10 torr of the He plus 2.5 torr of $F_{2}$ simulate the vibrational deactivation due to $\mathrm{NF}_{3}$. The specific heat of He is less than that of $\mathrm{NF}_{3}$, but the cemperature rise is not: very large. 
The results of the calculation are shown in Figs. 5.2 to 5.4. Figure 5.2 shows the density of the major species. $\mathrm{H}_{2}$ is consumed by reacting with $\mathrm{F}$ atoms and the amount of $\mathrm{HF}$ formed equals the amount of $\mathrm{H}_{2}$ consumed. The model assumes that all of the HF is formed as HF.1, most of it in the first two microseconds. Vibrational deactivation via reactions 3 and 4 then converts HF.1 to HF.0. The consumption of F atoms is clearly seen in Fig. 5.3. The density of $\mathrm{H}$ atoms is the mitror image of the $F$ atoms, an obvious result of reaction 1. Figure 5.4 shows the rate of formation of $\mathrm{HF}$ from reaction 1 and the rate of vibrational deactivation from reaction 3. The other reactions are insignificant under the chosen conditions. The curvature in the rate for reaction 1 in the first 2 microseconds is due to the rise in temperature from 300 to $380 \mathrm{~K}$. The laser output, in the steady state, is proportional to the rate of reaction 1 minus the rate of reaction 3 . Figure 5.4 is quite similar to the results of the analytic model shown in Fig. 4.5, except that $F_{L g} .5 .4$ is a semilogarithmic plot and the vibrational relaxation is smaller berause more HF was formed with the larger initial dissociation assumed for Fig. 4.5.

5.4.3 Simulation of the $\mathrm{F}_{2}+\mathrm{H}_{2}$ Chain Reaction We now return to the $\mathrm{F}_{2}+\mathrm{H}_{2}$ reaction with the appropriate rate in reaction?. The results of the calculation, shown in Figs 5.5 to 5.8, are quite difterent from those of Figs. 5.2 to 5.4. Figure 5.5 shows that nearly all of the $\mathrm{H}_{2}$ and $\mathrm{F}_{2}$ are constmed and create about 20 times as much $\mathrm{HF}$ 
as lihe previous roantion. This is accompanied by a rise in tomperature to nearly $5000 \mathrm{~K}$ as shown in lip. 5.6. The temperature would be lower iff lasim; occurred becalse a significant fraction of the hear of reaction would be lost as radiation. On the other hand, the calculation neglects the energy deposited by the electric discharge. It is rypically comparable to that losi by radiation.

Figure 5.7 shows the densities of $\mathrm{F}$ and $\mathrm{H}$ atoms. Initially the F atoms disappear as in Fig. 5.3. By about 1 microsecond the density of $\mathrm{H}$ atoms has built up to where reaction 2. replaces $F$ atoms as fast as they are consumed by reaction 1. At this point the reaction has reached steady state and $\mathrm{F}_{2}$ and $\mathrm{H}_{2}$ are consumed at an equal rate, as shown in Fig. 5.5 and especially in Fig. 5.8, where the net rates for reactions 1 and 2 are virtually identical from 1.5 to 3.5 microseconds. The small difference in rates between 4 and 5 microseconds is due to rapid dissociation of $F_{2}$ to give additional $F$ atoms. The additional $F$ atoms cause an increase in the rate of reaction 1 until it creates enough $B$ atoms to bring up the rate of reaction 2. After 4.5 III icroseconds both rates fall off mostly because the temperature has risen to where reactions 1 and 2 are approaching equilibrium.

If this reaction were pumping a laser at steady state the pulse shape would be proportional to the sum of 
the rates of roactions 1 and 2 in Fig. 5.8, up to the point that the vibrational. deactivation rates of reactions 3 and 4 become comparable. This occurs at about 2 microseconds. The laser pulse differs in shape, duration, and magnitude from the $\mathrm{NF}_{3}+\mathrm{H}_{2}$ laser pulse. All three differences come from the fact that the $\mathrm{F}_{2}+\mathrm{H}_{2}$ reaction is a chain and converts virtually a11 of the $\mathrm{H}_{2}$ and $\mathrm{F}_{2}$ to $\mathrm{HF}$. In the $\mathrm{NF}_{3}+\mathrm{H}_{2}$ reaction the amount of $\mathrm{HF}$ cannot exceed the initial density of $\mathrm{F}$ atoms. In these two calculations the initial density of $\mathrm{F}$ atoms was about $6 \%$ of the total $\mathrm{H}_{2}$ density. In the chain reaction the total amount of HF formed is about 20 times larger, resulting in a larger magnitude pulse. The duration of the $\mathrm{NF}_{3}+\mathrm{H}_{2}$ laser pulse is set by consumption of $F$ atoms. In the chain, $F$ atoms are repiaced by reaction 2 and the pulse lasts longer. Finally, the peak of the laser pulse occurs later in the chain reaction because the increased rate due to increased temperature more than compensates for consumption. As a result, the pulse shape for the $\mathrm{F}_{2}+\mathrm{H}_{2}$ laser differs from that of the $\mathrm{NF}_{3}+\mathrm{H}_{2}$ laser.

Vibrational relaxation is much more important in the chain reaction. It is relatively unimportant in the $\mathrm{NF}_{3}+\mathrm{H}_{2}$ laser because the $\mathrm{F}$ atoms are consumed tefore vibrational relaxation can uccur and also because the density of $H F$ Iemains relatively small. In the chain reaction the pulse duration is longer beciuse reaction 2 replaces the $F$ atoms consumed by reaction 1. As a result, the pulse 
can be terminated by vibrational doactivation. In addition, tho vibrational rate? is larger becillse more $\mathrm{HF}$ is created and because of the large: temperature rise. The net result is that in the chain reaction the laser prise is cerminated by vibrational deactivation before al1 of the reactants are consumed, Examination of Fig. 5.5 and 5.8 shows that the laser pulse terminates at about 2 microseconds. In Fig. 5.5, the density of HF.I is seen to peak at about 3.1 microseconds and then fall off. This is due to vibrational deactivation. The laser pulse, assuming steady state, would terminate when the slope of the HF,O curve matches that of HF.I (at abcut

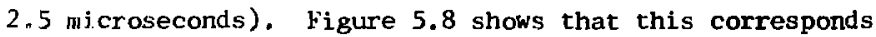
to the time when the vibrational deactivation rate becomes comparable to the rate of generation of $\mathrm{HF}$. To summarize, the kinetics of the $\mathrm{NF}_{3}+\mathrm{H}_{2}$ laser is dominated by consumption of $F$ atoms, and vibrational deactivation plays a secondary role. The kinetics of the $\mathrm{F}_{2}+\mathrm{H}_{2}$ laser is much different because reaction 2 replaces the $\mathrm{F}$ atom consumed in reaction 1 . The amount of $\mathrm{HF}$ formed greatly exceeds the initial density of $F$ atons resulting in a much larger laser pulse. Vibrational deactivation terminates the laser pulse because $\mathrm{HF}$ is generated for a longer time and in larger quantities. 
T2. He LASER SIMULATIOA

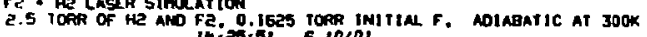

I4:zS;Gi S rolot

\begin{tabular}{|c|c|c|c|c|c|c|c|c|}
\hline 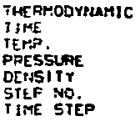 & $\begin{array}{r}\text { VARJAELES } \\
\text {.0E } 000 \\
3.000 E+02 \\
1.516 E+01 \\
8.105 E-07 \\
1.000 E-16\end{array}$ & $\begin{array}{r}.0 E+00 \\
3.000 E+02 \\
1.516 E+01 \\
0.105 E-07 \\
1.000 E-16\end{array}$ & $\begin{array}{r}2.623 E-12 \\
3.000 E+02 \\
1.51 E E+C 1 \\
8.105 E-07 \\
11 \\
5.961 E-12\end{array}$ & $\begin{array}{r}5.7005-10 \\
3.600 E+02 \\
1.516 E+01 \\
6.105 E-07 \\
1.529 E-10\end{array}$ & $\begin{array}{c}9.464 E-09 \\
3.005 E+02 \\
1.519 E+01 \\
8.105 E-07 \\
31 \\
2.363 E-09\end{array}$ & $\begin{array}{r}5.000 E-00 \\
3.02 E E+02 \\
1.530 E+01 \\
9.105 E-07 \\
1.145 E-08\end{array}$ & $\begin{array}{c}1.000 E-07 \\
3.057 E+0 z \\
1.545 E+01 \\
6.105 E-07 \\
3.065 E-00\end{array}$ & $\begin{array}{r}1.500 E-07 \\
3.009 E-02 \\
1.581 E+01 \\
9.105 E-07 \\
3.065 E-0 \mathrm{E}\end{array}$ \\
\hline $\begin{array}{l}\text { SLLECT } \\
\text { HE } \\
\text { FE } \\
\text { HF. I } \\
\text { H. D } \\
\text { F } \\
\text { H }\end{array}$ & $\begin{array}{r}\text { ENTRAT L IONS } \\
1.336 E=07 \\
1.33 E E-07 \\
.0 E+00 \\
.0 E+00 \\
\text { B. } 6 \text { BSE-09 } \\
.0 E+00\end{array}$ & $\begin{array}{r}\text { IN MOLES/CE } \\
1.336 E-07 \\
1.33 E E-07 \\
.0 E \circ 00 \\
.0 E+00 \\
9.685 E-09 \\
.0 E+00\end{array}$ & $\begin{array}{l}1.336 E-07 \\
1.336 E-07 \\
2.110 E-14 \\
7.309 E-21 \\
8.606 E-09 \\
2.110 E-14\end{array}$ & $\begin{array}{l}1.336 E-07 \\
1.336 E-07 \\
4.591 E-12 \\
3.459 E-16 \\
0.681 E-09 \\
4.590 \mathrm{~F}-12\end{array}$ & $\begin{array}{l}1.335 E-07 \\
1.336 E-07 \\
6.797 E-11 \\
7.58 E E-14 \\
6.618 E-09 \\
6.785 E-11\end{array}$ & $\begin{array}{l}1.332 E-07 \\
1.336 E-07 \\
3.984 E-10 \\
2.604 E-12 \\
\text { B. } 392 E-09 \\
3.940 E-10\end{array}$ & $\begin{array}{l}1.32 B E-07 \\
1.336 E-07 \\
7.699 E-10 \\
1.022 E-11 \\
7.914 E-09 \\
7.719 E-10\end{array}$ & $\begin{array}{l}1.325 E-07 \\
1.336 E-07 \\
1.176 E-09 \\
2.259 E-11 \\
7.552 E-09 \\
1.134 E-09\end{array}$ \\
\hline
\end{tabular}

FATES TMOL/SEC-CC, FRGM SELECTED REACTIONS

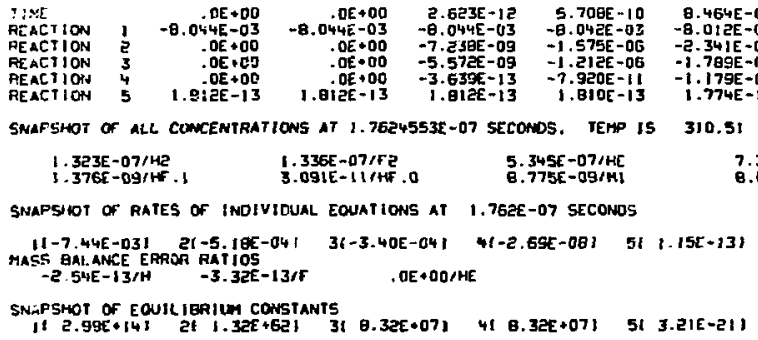

Table 5.1 Sample tabular output page from the MINOTAR code. These results were generated using the input shown in Table 5.2. 


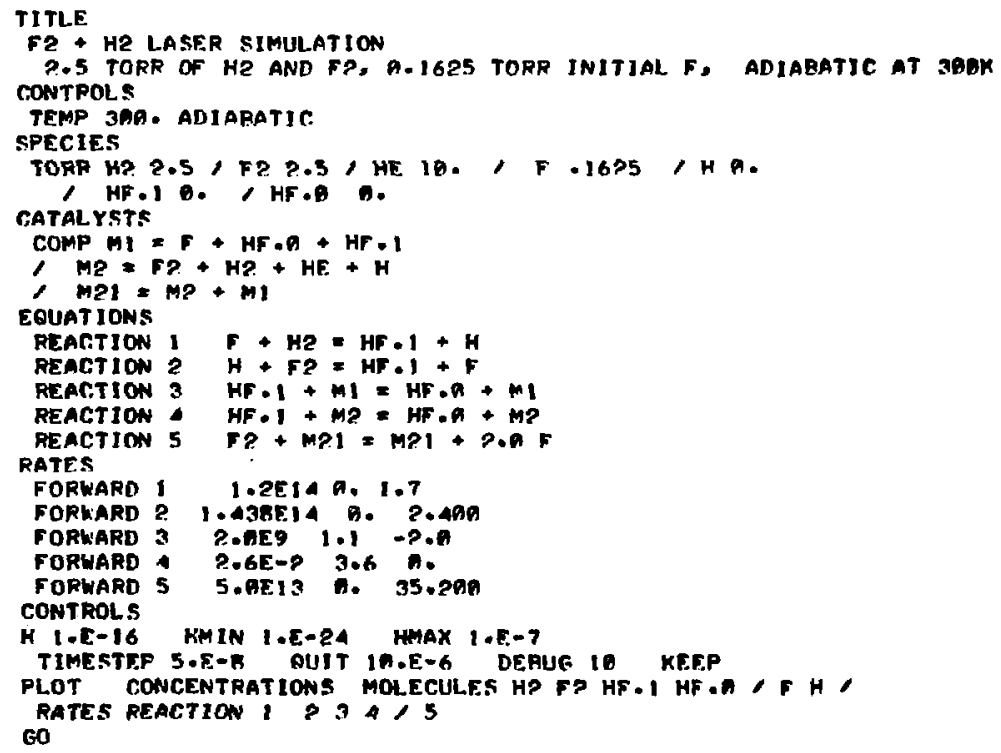

Table 5.2 Sample input for the MINOTAR code. 
I Fe HE LASER SIMUATION

2.5 TORR OF HE AND FE. 0.I6AS TOAR INITIAL F. MOIABATIC AT 300K COMPUTED RATE EQUATIÓNS $14: 25: 51$ i 10,01

Pact 5

\begin{tabular}{|c|c|c|c|c|c|c|}
\hline 0 othe & $1 / 0 \mathrm{~T}$ & $\begin{array}{r}-1.00 . \mathrm{kf} \\
+\quad 1.00 \cdot \mathrm{kf}\end{array}$ & $\begin{array}{l}1: \bullet C H E \\
11: C H\end{array}$ & $\begin{array}{l}\text { JeF } \\
\text { Jets. I }\end{array}$ & It & 1 \\
\hline a dife & irot & 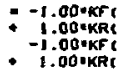 & 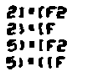 & 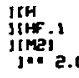 & $\prod_{\text {in }}$ & $\sum_{101}^{3} 1.01$ \\
\hline o Dif & J/DT & 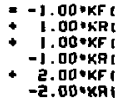 & 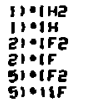 & 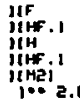 & 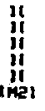 & $\begin{array}{c}j \\
j \\
j \\
j\end{array}$ \\
\hline D DIH & MOT & $\begin{array}{r}1.000 K F ! \\
-1.00 \times k R ! \\
-1.00 \times k F ! \\
+\quad 1.00 \text { KRt }\end{array}$ & 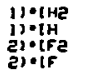 & $\begin{array}{l}\text { jtF } \\
j t+.1 \\
\text { lth } \\
\text { ltH. . }\end{array}$ & $\begin{array}{l}\text { it } \\
\text { it } \\
\text { it }\end{array}$ & ! \\
\hline 0 oth. 1 & Hor & 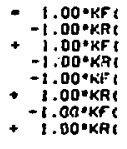 & 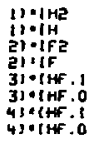 & 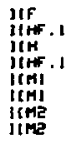 & $\begin{array}{l}\text { di } \\
\text { yi } \\
\text { ji } \\
\text { ji } \\
\text { it }\end{array}$ & $\begin{array}{l}\mathbf{3} \\
\mathbf{1} \\
\mathbf{j} \\
\mathbf{j} \\
\mathbf{j}\end{array}$ \\
\hline O DIHF. 0 & Wor & $\begin{array}{r}1.00 \cdot K F t \\
-1.00^{\circ K F t} \\
-1.00^{\circ K F t} \\
-1.00^{\circ} \mathrm{KFt}\end{array}$ & 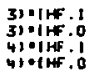 & $\begin{array}{l}\text { Jini } \\
\text { limi } \\
\text { lime } \\
\text { if }\end{array}$ & 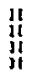 & $\begin{array}{l}j \\
\vdots \\
1\end{array}$ \\
\hline
\end{tabular}

Table 5.3 Chemical kinetic rate equations derived by the MINOTAR computer code using the input of Table 5.2. 


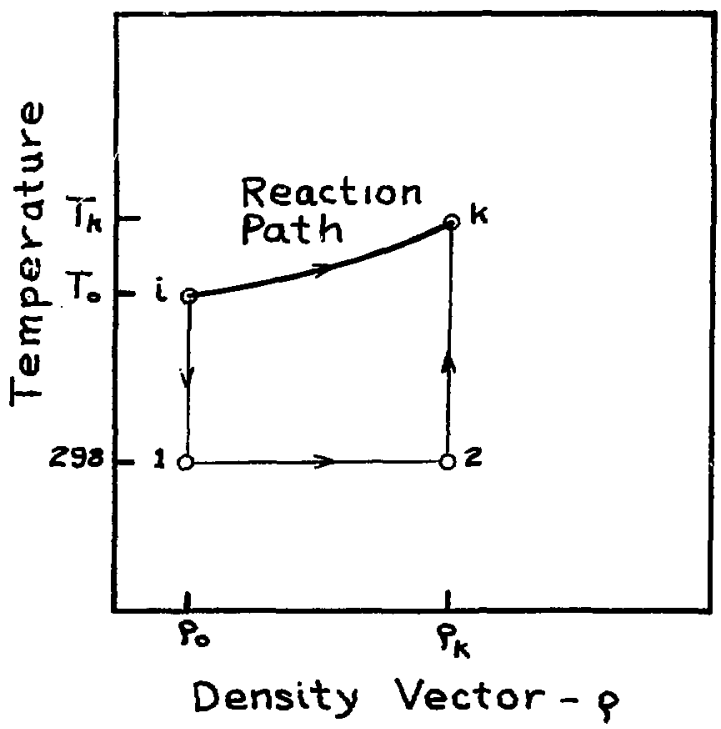

Fig. 5.1 The thermolynamic p:th for calculating the tempereture in the MINOTAR cole. The density rector is if function of the dens: t:es of all species. The :nit:ial stidic i.s "i.", the state at time step k is "k". 


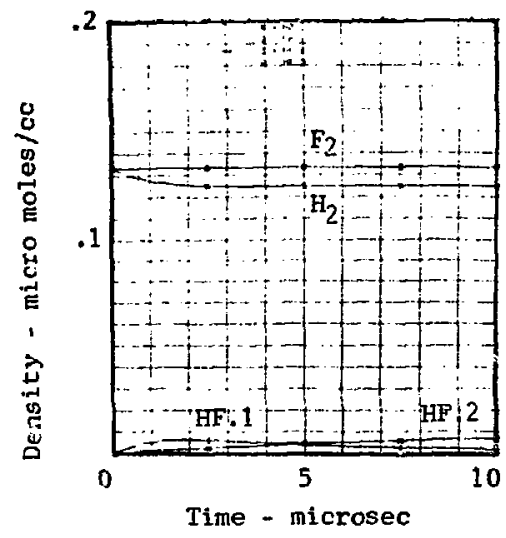

Fig. 5.2 Densities of the major species in the $\mathrm{NF}_{3}+\mathrm{H}_{2}$ simulation.

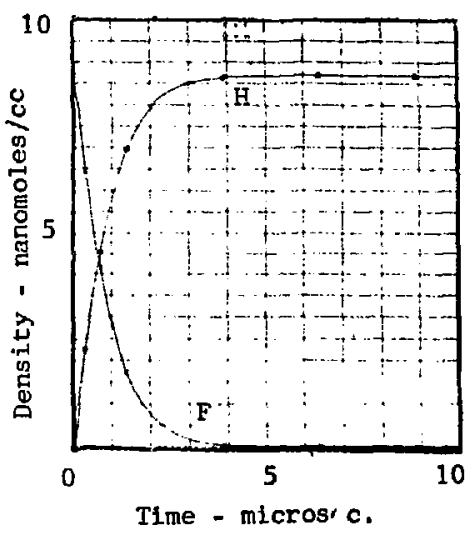

Fig. 5.3 Densities of the atomic radicals, $H$ and $F$, in the $\mathrm{NF}_{3}+\mathrm{H}_{2}$ simulation. 


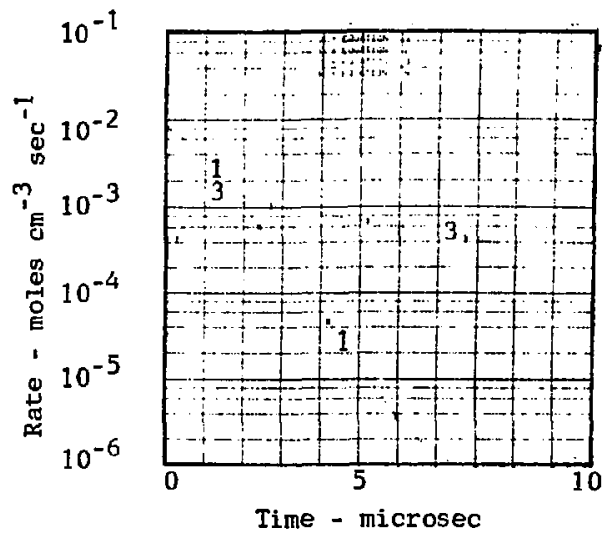

Fig. 5.4 Rates of reactions 1 and 3 in the $\mathrm{NF}_{3}+\mathrm{H}_{2}$ simulation. 


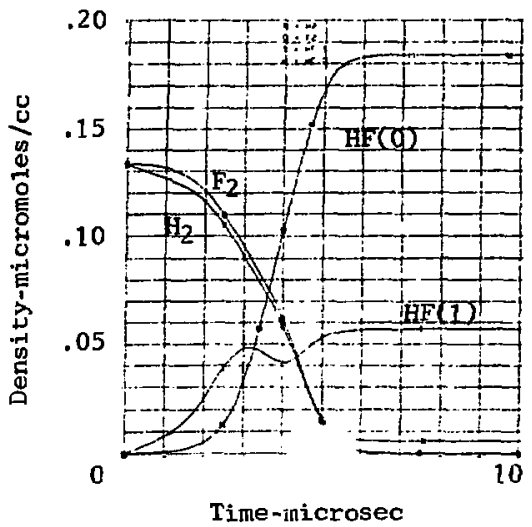

Fig. 5.5 Densities of the major chemical species in the $\mathrm{H}_{2}+\mathrm{F}_{2}$ chain reaction.

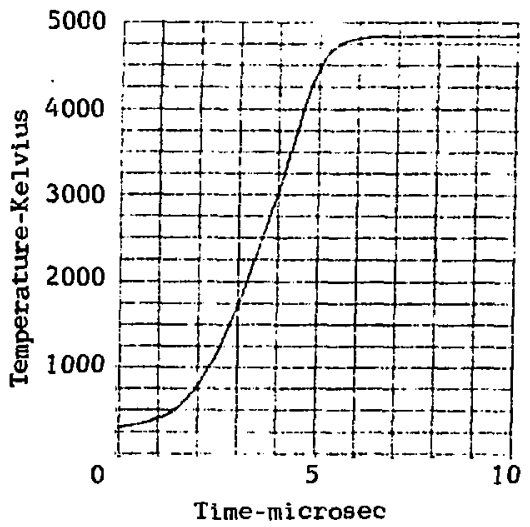

Fig. 5.6 Temperature change in the $\mathrm{H}_{2}+\mathrm{F}_{2}$ chain reaction. 


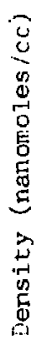

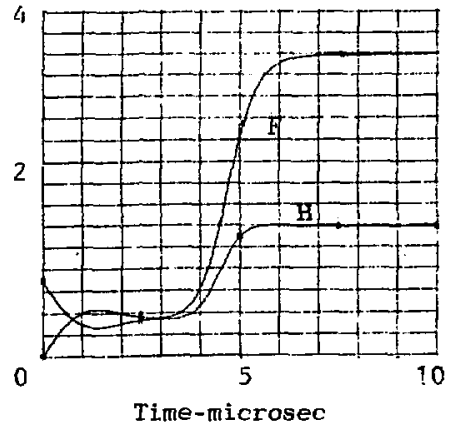

Fig. 5.7 Densities of the radicals, $H$ and $F$, in the $\mathrm{H}_{2}+\mathrm{F}_{2}$ chain reaction.

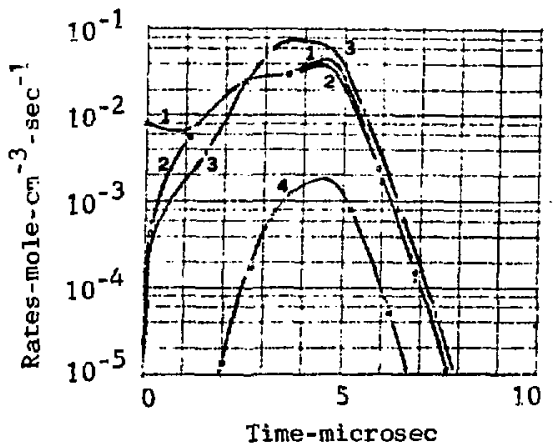

FiB. 5.8 Rates of the reactions in the $\mathrm{H}_{2}+\mathrm{F}_{2}$ chain reaction. 
6. LASER EQUATIONS IOOR A MORE DETAJIED MODEL.

\subsection{Introduction}

The laser model of previous sections has assumed that lasing occurs betwen two vibrational levels and ignored the rotational structure of these levels. It is possible, in a numerical model, to assume rotational equilibrium at the translational temperature within each vibrational level. Then the gain for each P-branch transition can be calculated, along with the threshold population inversion for each transition. Between any two vibrational levels one transition will have the smallest threshold inversion. If a steady-state laser model is used, the population difference will be clamped by that threshold illversior. Other P-branch transjitions between the same vibrational levels will then be below threshold, and will be unable to lase.' Therefore, a model which assumes rotational equilibrium and a steady-state laser will have only one $\mathrm{P}$-branch transition lasing between two vibrational levels at any time. As populations and temperatures change the threshold inversions also change. From time to time a nuilasing transition will develop a threshold inversion smaller than that of the transition that is currently lasing. The lasing then will switch to the new transition. This j.s referred to in the literature as J-shifting (Emanue1, 1971; Chester, 1970).

Earlier studies used this model for the HF laser (Emanuel and Whitier, 1972; Lurner, Adams, and Emanuel, 1973). 
The nexl obvious improvement in an HF laser model was to separate the rotational states and allow thell to relax towards erfuilibrium with realistic relaxation rates. (Assuming rotational equilibriun is equivalent to assuming an infinite rotational relaxation rate.) If the rotational states are separated, several P-branch transitions can lase between two vibrational levels at any time, in agreement with experiment.

The original plan was to treat rotational states separately using a steady-state laser model for each transition. Upon examination, it appeared to be just as simple to use laser rate equations in the computer code. Preliminary calculations showed that certain assumptions implicit in laser rate equations might be violated in the HF laser so semi-classical laser equations were used.

The semi-classical laser equations are also rate enuations in the sense that they involve time derivatives. The term "laser rate equations" used here is a part of laser jargon. It refers specifically to treating the laser in cerms of a one equation for the population inversion and another equation for the photon density. "Laser rate equations" can be derived from the Einstein treatment of stimulated emission (Pantell and Puthoff, 1969). "Semiclassical laser equations" are derived by treating the population inversion quantum mechanically and the radiation field classically by means of Maxwell's equations (Shirley, 1968; Davis. 1963). This results in differential equations 
for the electric field, the dielectric polarization, the phase angle between them, and the population inversion. "Semi-classical laser equations" are equivalent to laser rate equations when the dielectric polarization is damped much more rapidy than the electric field.

Laser cascades occur in the HF laser because high vibrational numbers are pumped. The molecule then goes to the vibrational ground state by emitting several photons. The selection rules require that the laser transitions be P-branch transitions and that the molecule drop one vibrational level at a time. This is a cascade. Adjacent cascades are soupled by rotational relaxation, but not by radiation except in the unlikely case that there is also R-branch lasing.

Haken (1965) has treated cascades extensively using laser rate equations. When semi-classical equations are used there is a possibility that the electric fields or polarizations of two non-resonant transitions may interact. Haken's work does not treat this problem. Therefore it was necessary to derive semi-classical. laser equations for a cascade. It is shown that in general there can be radiation from the highest level to the lowest in a cascade, but this is prevented in a diatomic molecule by the selection rules. Thus the cascade can be treated as two isolated transitions coupled only by a shared molecular level. In order to understand the relaxation oscillations in the laser pulse there is an extensive treatment of the stability of the varjous linser equalions. 


\subsection{Herivation of Semi-C1atsisical Laser Equations.}

for a Thiee-Love1 Cilscado

Semiclassical equations to describe a two-level laser are: well known (Shirley, 1968; Davis, 1963). The two-level' laser has a single transition at a frequency defined by the encrgy difference of the two levers, with some modification of the frequency if the cavity is tuned slightly of the atomic resonance. If, for simplicity, we restrict ourselves to consideration of a single mode of the cavity and assume that the gain medium is spatially uniform, we get equations of motion for four variables: the electric field, the electric polarization, the phase angle between the field and the polarization, and the inversion. There is also a fifth equation for the phase angle of the electric field relative to the time base. This is of little importance unless we are considering noise or coherence time,

In a cascade there are a number of transitions coupled by common levels. The simplest case is a three-level cascade. (This is not to be confused with the three-level system used to describe the ruby laser. In that system the third level is part of the pumping scheme for a single laser transition between two leve1s.) The three-level cascade is shown in Fig. 6.1. The two-laser transitions, at different frequencies, are coupled by a common atomic or molecular level, level 2. It would be convenient to treat each transition as an independent transition coupled only by the population of the common level. (We will show later that the HF laser cannot have transitions directly from level 3 to leve1 1.) We can then use the well-known equations for a two-level laser. In order to determine the inceraction 
between the two transitions, it is necessary to derive equations for a three-level cascade.

Begin by assuming electric fields of the form $e_{1}(t) e^{-i(\omega} 1^{\left.t+\phi_{1}\right)}$ and $\left.e_{2}(t) e^{-i(\omega} t+\phi_{2}\right)$, where $e_{1}$ and $e_{2}$ are the envelope of the electric field and vary slowly compared to the optical frequencies $\omega_{1}$ and $\omega_{2}$. Since we wish to compare the calculation with experiments, we may consider only the envelope and neglect the rapidly oscillating terms, $e^{-i \omega t}$. Since the laser is nonlinear we must consider terms with frequencies $\omega_{1} \pm \omega_{2}$. Terms at the sum frequency will average to zero when integrated over the detector response time. In the case of $\mathrm{HF}$ vibrational-rotational transitions the difference frequency will exceed $10^{11} \mathrm{sec}^{-1}$ so we can also neglect it. We will also neglect mode-locking since the relevant frequencies are at least an order of magnitude above the detector response, and because it will be much simpler to consider a single mode on each transition. Javan (1957), Bloembergen and Shen (1964) and Feld and Javan (1969) have done very general derivations of the three-level laser. The derivation used here is an algebraically simpler one based on a generalization of the derivation of a two-level laser by Shirley (1968). The algebr: : simplicity results from the use of raising and lowering operators.

Let the energies of the states be $\mathrm{H}_{33}>\mathrm{H}_{22}>\mathrm{H}_{11}$, and define $h_{\omega}=\mathrm{H}_{22}-\mathrm{H}_{11}, h_{2} \omega_{2}=\mathrm{H}_{33}-\mathrm{H}_{22}$, and $\hbar_{\omega_{3}}=\mathrm{H}_{33}-\mathrm{H}_{11}=$ $x_{2}\left(w_{1}+w_{2}\right)$. The molecular states can be represented as vectors 


$$
\psi_{1}=\left(\begin{array}{l}
0 \\
0 \\
1
\end{array}\right), \quad \psi_{2}=\left(\begin{array}{l}
0 \\
1 \\
0
\end{array}\right) \text { and } \psi_{3}=\left(\begin{array}{l}
1 \\
0 \\
0
\end{array}\right)
$$

The raising operator: for the inolecule are represented by $\sigma_{1}^{+}=\left(\begin{array}{lll}0 & 0 & 0 \\ 0 & 0 & 1 \\ 0 & 0 & 0\end{array}\right), \quad \sigma_{2}^{+}=\left(\begin{array}{lll}0 & 1 & 0 \\ 0 & 0 & 0 \\ 0 & 0 & 0\end{array}\right), \quad$ and $\quad \sigma_{3}^{+}=\left(\begin{array}{lll}0 & 0 & 1 \\ 0 & 0 & 0 \\ 0 & 0 & 0\end{array}\right)(6.2)$ where $\sigma_{1}^{+}$raises the molecule from state 1 to state 2 , and $\sigma_{2}^{+}$raises from state 2 to state 3 , etc. The corresponding lowering operators are $\sigma_{i}^{-}=\left(\begin{array}{lll}0 & 0 & 0 \\ 0 & 0 & 0 \\ 0 & 1 & 0\end{array}\right), \quad \sigma_{2}^{-}=\left(\begin{array}{lll}0 & 0 & 0 \\ 1 & 0 & 0 \\ 0 & 0 & 0\end{array}\right), \quad$ and $\quad \sigma_{3}^{-}=\left(\begin{array}{lll}0 & 0 & 0 \\ 0 & 0 & 0 \\ 1 & 0 & 0\end{array}\right)(6.3)$ Number operators giving the number in each state can now be defined: $\mathrm{n}_{1}=\sigma_{1}{ }^{-} \sigma_{1}^{+}=\sigma_{\sigma_{3}}{ }^{-} \sigma_{3}{ }^{+}, \mathrm{n}_{2}=\sigma_{1}{ }^{+} \sigma_{1}{ }^{-}=\sigma_{2}{ }^{-} \sigma_{2}{ }^{+}$and $n_{3}=\sigma_{2}{ }^{+}{ }^{-}+\sigma_{3}{ }^{+} a_{3}^{-}$. These have the property that $n_{1} \psi_{2}=n_{1} \psi_{3}=0$, etc. They are represented by the matrices $n_{1}=\left(\begin{array}{lll}0 & 0 & 0 \\ 0 & 0 & 0 \\ 0 & 0 & 1\end{array}\right), \quad n_{2}=\left(\begin{array}{lll}0 & 0 & 0 \\ 0 & 1 & 0 \\ 0 & 0 & 0\end{array}\right), \quad$ and $n_{3}=\left(\begin{array}{lll}1 & 0 & 0 \\ 0 & 0 & 0 \\ 0 & 0 & 0\end{array}\right)$ We also let $a_{1}^{+}$and $a_{1}$ be the creation and annihilation operators for the radiation at the transition with frequency $\omega_{1}$. The cavity mode frequency for this transition will be ${ }^{\omega_{c 1}}$, which may differ slightly from $\omega_{1}$. The number of photons in the mode is given by the operator $a_{1}^{+} a_{1}$. Corresponding operators are $a_{2}^{+} a_{2}$ and $a_{3}^{+} a_{3}$ for the modes at frequencies $\omega_{c 2}$ and $\omega_{c 3}$. 
We wish to write a Hamiltonian for a system of molecules, therefore we will have to sum over all molecules. Setting the ground state energy to zero we obtain the Hamil t onian

$$
\begin{aligned}
& H=h_{c 1} a_{1}^{+} a_{1}+h_{c} a_{2}^{+} a_{2}+h_{\omega} c_{3} a_{3}^{+} a_{3} \\
& +\sum_{j} \hbar \omega_{1} n_{2 j}+\sum_{j} \hbar \omega_{3} n_{3 j} \\
& +h b_{1} \sum_{j}\left(o_{1 j}^{+} a_{1}+\sigma_{1 j}^{-} a_{1}^{+}\right)+h b_{2} \sum_{j}\left(o_{2 j}^{+} a_{2}+o_{2 j}^{-} a_{2}^{+}\right) \\
& +\hbar b_{3} \sum_{j}\left(\sigma_{3 j}+a_{3}+\sigma_{3 j}{ }^{-} a_{3}^{+}\right)
\end{aligned}
$$

where the first three terms are the Hamiltonian for the radiation, the second two are the Hamiltonian for the system of molecules, and the last three terms are the interaction Hamiltonian. The factors $b_{1}, b_{2}$ and $b_{3}$ are proportional to the dipole matrix elements. We have dropped terms in the interaction Hamiltonian of the form $\sigma_{1}^{+} a_{1}^{+}$and $\sigma_{1}^{-} a_{1}$ because they represent creation (annihilation) of a photon along with increasing (decreasing) the energy of the molecule, and are thus highly improbable. Neglecting these terms is equivalent to the rotating wave approximation.

We obtain differential equations for each operator from the Heisenberg equation of motion

$$
\text { in } \frac{d A}{d t}=[A, H]+i \hbar \frac{\partial A}{\partial t}
$$


where the first term on the ripht is the commut ator $[A, H]=$ (AH - HA), and the second terta vanishes except when $A$ has an explicit time dependence. When the Hamiltonian of Eq. 6.5 is inserted in Eq. 6.6 the conmutators are rendi $1 y$ evaluated and are tabulated in Appendix C. Wo obtain the lollowing,

equations:

$$
\begin{aligned}
& \dot{i}_{1}=w_{r l} a_{1}+b_{1} \sum_{j} a_{i j}^{-} \\
& \dot{i}_{2}=\omega_{c 2} a_{2}+b_{2} \sum_{j} a_{2 j} \\
& \stackrel{\circ}{i a_{3}}=w_{c \cdot 3} a_{3}+b_{3} \sum_{j} o_{3 j}^{-} \\
& i_{i j}^{-}{ }_{1 j}=w_{1} \sigma_{1 j}^{-}+b_{1}\left(n_{1}-n_{2}\right) a_{1}+b_{2} \sigma_{3 j}^{-} a_{2}^{+}-b_{3} \sigma_{2 j}^{+} a_{3} \\
& \dot{i}_{\check{\sigma}_{j}}^{-}=w_{2} \sigma_{2 j}^{-}+b_{2}\left(n_{2}-n_{3}\right) a_{2}-b_{1} \sigma_{3 j}^{-} a_{1}^{+}+b_{3} \sigma_{1 j}^{+} a_{3} \\
& \stackrel{\circ}{\circ}_{3 j}=w_{3} \sigma_{3 j}^{-}-\dot{o}_{1} \sigma_{2 j}^{-} a_{1}+b_{2} \sigma_{1 j} a_{2}+b_{3}\left(n_{1}-n_{3}\right) a_{3} \\
& i \dot{n}_{1 j}=b_{1}\left(\sigma_{1 j}^{-} a_{1}^{+}-\sigma_{1 j}^{+} a_{1}\right)-b_{3}\left(\sigma_{3 j}^{+} a_{3}-\sigma_{3 j} a_{3}^{+}\right) \\
& i a_{2 j}=-b_{1}\left(\sigma_{1 j}^{-} a_{1}^{+}-\sigma_{1 j}^{+} a_{1}\right)+b_{2}\left(\sigma_{2 j}^{-} a_{2}^{+}-\sigma_{2 j}^{+} a_{2}\right) \\
& i \dot{n}_{3 j}=-b_{2}\left(o_{2 j}^{-} a_{2}^{+}-\sigma_{2 j}^{+} a_{2}\right)+b_{3}\left(a_{3 j}^{+} a_{3}-o_{3 j} a_{3}^{+}\right) \text {. }
\end{aligned}
$$

The summations in equations 6.7 and 6.8 have dropped out because operators for one molecule commute with those for other molecuies. Analogous equations can be obtained for $\mathrm{a}^{+}$and $\mathrm{o}^{+}$operators.

In order to get macroscopic variables we must take expectation values of Eqs. 6.7 to 6.9 . To save space we will drop some of the equations for transition 2, and obtain 
$\frac{d}{d t}\left\langle a_{1}\right\rangle=-i \omega_{c 1}\left\langle a_{1}\right\rangle-i b_{1} \sum_{j}\left\langle\sigma_{1 j}^{-}\right\rangle$

$\frac{d}{d t}\left\langle\sigma_{1 j^{-}}^{-}\right\rangle=-i \omega\left\langle\sigma_{1 j^{-}}^{-}-i b_{1}\left\langle\left(n_{1}-n_{2}\right) a_{1}\right\rangle-i b_{2}\left\langle\sigma_{3 j}^{-} a_{2}^{+}\right\rangle\right.$

$$
+i b_{3}<o_{2 j}^{+} a_{3}>
$$

$\frac{d}{d t}\left\langle n_{1 j}\right\rangle=-i b_{1}\left\langle\sigma_{1 j}^{-} a_{1}^{+}\right\rangle+i b_{1}\left\langle o_{1 j}^{+} a_{1}\right\rangle+i b_{3}\left\langle o_{3 j}^{+} a_{3}\right\rangle$

$-i b_{3}\left\langle\sigma_{3 j} a_{3}^{+}>\right.$

$\frac{d}{d t}\left\langle\pi_{2 j}\right\rangle=i b_{1}\left\langle\sigma_{1 j}^{-} a_{1}^{+}\right\rangle-i b_{1}\left\langle\sigma_{1 j}^{+} a_{1}\right\rangle$

$$
\text { - } i b_{2}\left\langle\sigma_{2 j}^{-} a_{2}^{+}\right\rangle+i b_{2}\left\langle\sigma_{2 j}^{+} a_{2}\right\rangle
$$

$$
\begin{aligned}
\frac{d}{d t}\left\langle o_{3 j}^{-}\right\rangle & =-i \omega_{3}\left\langle o_{3 j}^{-}\right\rangle-i b_{3}\left\langle\left(n_{1}-n_{3}\right) a_{3}\right\rangle \\
& +i b_{1}\left\langle o_{2 j}^{-} a_{1}\right\rangle-i b_{2}\left\langle o_{1 j}^{-} a_{2}\right\rangle
\end{aligned}
$$

At this point we encounter a difficulty because $\left\langle 0_{1}^{-} a_{1}^{+}\right\rangle\left\langle\left\langle 0_{1}^{-}\right\rangle\left\langle a_{1}^{+}\right\rangle\right.$. However, if we have an intense radiation field the quantum number for the harmonic oscillator is large and by the correspondence principle should behave classically. Therefore we make a semi-classical approximation $\left\langle\sigma_{1}^{-} a_{1}^{+}\right\rangle=\left\langle\pi_{1}^{-}\right\rangle\left\langle a_{1}^{+}\right\rangle$, and similar approximations for other products (Shirley, 1968).

We can now make the connection with macroscopic. variabies.

$$
A_{1}=\left\langle a_{1}\right\rangle e^{j \cdot \Omega_{1} t}
$$


where $\Omega_{1}$ is the frequency of the electric tiold and may be different from both $w_{1}$, the molecular resonance frequency, and $\omega_{c l}$, the cavicy resonance frequency. We also have

$$
A_{1}^{*}=\left\langle n_{1}^{+}\right\rangle e^{-i \Omega_{1} t}
$$

and the product $A_{1}^{*} A_{1}$ is the number of photons in the radiatior. field. The variable $A_{1}$ is connected to the electric field by

$$
E_{1}(t)=\sqrt{\frac{h \omega_{c 1}}{2 E V}}\left(A_{1} e^{-i \Omega_{1} t}+c_{\cdot} c_{1}\right)
$$

The carrier frequency is handled so that $E_{1}(t)$ is the envelope of the electric field; $V$ is the volume of the system and $E$ the permittivity. We also derine macroscopic variables

$$
P_{1}=\sum_{j}\left\langle\sigma_{1 j}^{-}\right\rangle e^{i \Omega_{1} t}
$$

and

$$
P_{1}^{*}=\sum_{j}<0_{1 j}^{+}>e^{-i n_{1} t}
$$

which are connected to the envelope of the polarization by

$$
\rho_{1}=\frac{\mu_{12}}{v}\left(P_{1} e^{-i \Omega_{1} t}+c, c .\right) \text {. }
$$

with $\mu_{12}$ the electric dipole matrix element.

Finally, we have the populations

$$
\mathrm{N}_{1}=\sum_{j} \mathrm{n}_{1 j} \text { and } \mathrm{N}_{2}=\sum_{j} \mathrm{n}_{2 j} \cdot
$$

With macroscopic valialles defined, we take derivaidives of Eqs. 6.1.1, 6.13 and 6.15 and substitute in Eqs. 6.10, giving 


$$
\begin{aligned}
& \dot{A}_{1}=i\left(\Omega_{1}-\omega_{c 1}\right) A_{1}-i b_{1} P_{1} \\
& \dot{A}_{2}=i\left(\Omega_{2}-\omega_{c 2}\right) A_{2}-i b_{2} P_{2} \\
& \dot{A}_{3}=i\left(\Omega_{3}-\omega_{c 3}\right) A_{3}-i b_{3} P_{3} \\
& \left.\dot{P}_{1}=i\left(\Omega_{1}-\omega_{1}\right) P_{1}+i b_{1} A_{1} i N_{2}-N_{1}\right)-i b_{2} A_{2}^{*} P_{3}+i b_{3} A_{3} P_{2}^{*} \\
& \dot{P}_{2}=i\left(R_{2}-\omega_{2}\right) P_{2}+i b_{2} A_{2}\left(N_{3}-N_{2}\right)+i b_{1} A_{1}^{*} P_{3}-i b_{3} A_{3} P_{1}^{*} \\
& \dot{P}_{3}=i\left(R_{3}-\omega_{3}\right) P_{3}+i b_{3} A_{3}\left(N_{3}-N_{1}\right)+i b_{1} A_{1} P_{2}-i b_{2} A_{2} P_{1} \\
& \dot{N}_{1}=i b_{1}\left(P_{1}^{*} A_{1}-A_{1}^{*} P_{1}\right)+i b_{3}\left(P_{3} * A_{3}-A_{3}^{*} P_{3}\right) \\
& \dot{N}_{2}=-i b_{1}\left(P_{1}^{*} A_{1}-A_{1}^{*} P_{1}\right)+i b_{2}\left(P_{2}^{*} A_{2}-A_{2}^{*} P_{2}\right) \\
& \dot{N}_{3}=-i b_{2}\left(P_{2}^{*} A_{2}-P_{2} A_{2}^{*}\right)-i b_{3}\left(P_{3}^{*} A_{3} \cdot A_{3}^{*} P_{3}\right)
\end{aligned}
$$

(The equations for $A_{k}$ and $P_{k}$ contain factors $e^{i \omega_{k} t}$, but they are not shown because they can be factored out.)

Similar equations may be obtained for a two-level

system ,

$$
\begin{aligned}
& \dot{A}=i\left(\Omega-\omega_{C}\right) A-i b P \\
& \dot{P}=i(\Omega-\omega) P+i b A\left(N_{2}-N_{1}\right) \\
& \dot{N}_{1}=i b\left(P_{A}^{*}-A^{*} P\right) \\
& \dot{N}_{2}=-i b\left(P^{*} A-A * P\right)
\end{aligned}
$$

The population equatjons for the three level case (Eq. 6.16) are an obvious gencralization of the two-level equations $(6,17)$, but the polarization equations have terms of the form $b_{1} A_{1} P_{2}$ which represent a new physical effect. This is a type of two-photon interaction. The electric field at frequency $\omega_{1}$ interacts with the polarization at 
frequency $\omega_{2}$ to create a polarization at frequenty $\omega_{3}$, the sum of $\omega_{1}$ and $\omega_{2}$. The original polari\%ation was created by absorption of a photon at $\omega_{2}$, su the polarization at $\omega_{3}$ is he result of absorbing, two photons, the sum of whose frequencics is resonant with $\omega_{3}$. The photons need not be absorbed simultaneously; however, if we added damping terms to Eqs. 6.16 both photons would have to be absorbed in an interval shorter than the damping time. Similarly, the polarization at $\omega_{3}$ interacts with emission of a photon at $\omega_{1}$ or $\omega_{2}$ to give $a$ polarization at $\omega_{2}$ or $\omega_{1}$, respective1y. The net result is thit radiation on two of the frequencies pumps or depletes radiation on the third. This has been discussed extensively in the litera ure (Javan, 1957; Bloembergen and Shen, 1964; Feld and Javar, 1969; Haken, et al., 1965).

In the present case we have a simplification. Selection rules forbid dipole transitions at $\omega_{3}$ in the vibrational-rotational spectrum (the rule is $\Delta \mathbf{J}= \pm \mathbf{l}$ allowed and $\omega_{3}$ has $\Delta J= \pm 2$, which is forbidden), As a result, $b_{3}$ is zero and many of the coupling terms drop out. Most important, the second term in the equation for $\AA_{3}$ drops out, causing $A_{3}$ :o oscillate at a constant amplitude. It, reality, loss of photons will have the effect of adding a damping term so that $A_{3}$ will always be zero. If $b_{3}$ is finite but much smaller than $b_{1}$ and $b_{2}$, then $A_{3}$ will still be infinitesimal because the other transitions will clamp $\left(\mathrm{N}_{3}-\mathrm{N}_{1}\right)$ to 
the same order of magnitude as $\left(N_{2}-N_{1}\right)$ and $\left(N_{3}-N_{2}\right)$ and the damping term will be much larger than $b_{3} P_{3}$. This will be more clear after we have discussed the steady-state solutions.

The vanishing of $A_{3}$ is important because $b_{3}$ is in fact finite due to an allowed electric quadrupole transition, but $b_{3}$ will be several orders of magnitude weaker than $b_{1}$ or $b_{2}$. This situation is not unique to the HF chemical laser. Electric dipole transizions are allowed only between stites of opposite parity, and electric quadrupole transitions between states of the same parity. But quadrupo'e transitions are orders of magnitude weaker than dipole transitions. The factors $b_{1}, b_{2}$, and $b_{3}$ can all be of comparable magnitude in the special case where the states are mixtures of comparable fractions of even and odd parity.

Equations 6.16 give a comp' 2 te description of the three-level system, but it. is convenient to reduce the complex variables to real variables, and drop terms conaining $\mathrm{A}_{3}$.

$$
\text { Let } \begin{aligned}
& A_{1}=\theta_{1} e^{i \theta_{1}} \\
& b_{1} P_{1}=i p_{1} e^{i\left(\theta_{1}-\theta_{1}\right)} \\
& b_{2} P_{2}=i p_{2} e^{i\left(\theta_{2}-\theta_{2}\right)} \\
& P_{3}=i p_{3} e^{-i \theta_{3}}
\end{aligned}
$$


98

Note that $p_{1}$ and $q_{1}$ are defined so that absorption or emission of 1 light is a maximum when $\phi_{1}$ and $\phi_{2}$ are zero. When these expressions ar s substituted in Exp. 6.17 for the two-level system wo get six real equations

$$
\begin{aligned}
& \stackrel{\circ}{q}=p \cos \\
& \stackrel{\circ}{p}=b^{2} q\left(N_{-}-N_{1}\right) \cos \omega \\
& \stackrel{\circ}{N}_{1}=2 p q \cos \phi \\
& \stackrel{\circ}{N}_{2}=-2 p q \cos \\
& \stackrel{\circ}{\theta}=\Omega-w_{c}-(p / q) \sin \\
& \stackrel{\circ}{q}=\omega-\omega_{c}-\left[p / q+b^{2}(q / p)\left(N_{2} / N_{1}\right)\right] \sin
\end{aligned}
$$

When these are subst]'uted into Eds. 6.16 for the three-level system we obtain a larger set of real equations.

$$
\begin{aligned}
& \stackrel{q}{q}_{1}=p_{1} \cos \phi_{1} \\
& \stackrel{\circ}{\mathrm{q}}_{2}=\mathrm{p}_{2} \cos \phi_{2} \\
& \stackrel{\circ}{p}_{1}=b_{1}^{2} q_{1}\left(N_{2}-N_{1}\right) \cos \phi_{1}+b_{1} b_{2} q_{2} p_{3} \sin \left(\xi+\iota_{1}\right) \\
& \stackrel{\mathrm{p}}{\mathrm{p}}_{2}=\mathrm{b}_{2}^{2} \mathrm{q}_{2}\left(\mathrm{~N}_{3}-\mathrm{N}_{2}\right) \cos \mathrm{p}_{2}-\mathrm{b}_{1} \mathrm{~b}_{2} \mathrm{q}_{1} \mathrm{P}_{3} \sin \left(\epsilon+\phi_{2}\right) \\
& E=\theta_{1}+\theta_{2}+\theta_{3} \\
& {\stackrel{\circ}{p_{3}}}_{3}=-\left(b_{1} / b_{2}\right) q_{1} p_{2} \sin \left(c-\phi_{2}\right) \\
& +\left(b_{2} / b_{1}\right) q_{2} p_{1} \sin \left(\xi-\phi_{1}\right) \\
& \stackrel{\circ}{N}_{1}=2 p_{1} q_{1} \cos \phi_{1} \\
& \stackrel{\circ}{N}_{2}=2 p_{2} q_{2} \cos \phi_{2}-2 p_{1} q_{1} \cos \phi_{1} \\
& \stackrel{\mathrm{N}}{3}_{3}=-2 \mathrm{p}_{2} \quad \mathrm{q}_{2} \cos \phi_{2}
\end{aligned}
$$




$$
\begin{aligned}
& \ddot{\theta}_{1}=a_{1}-\omega_{c 1}-\left(p_{1} / q_{1}\right) \sin \Delta_{1} \\
& \dot{\theta}_{2}=s_{2}-\omega_{c 2}-\left(p_{2} / q_{2}\right) \sin \varphi_{2} \\
& \dot{\theta}_{3}=\omega_{3}-\Omega_{3}-\frac{b_{1} q_{1} D_{2}}{b_{2} p_{3}} \cos \left(6-s_{2}\right) \\
& +\frac{b_{2} q_{2} p_{1}}{b_{1} p_{3}} \cos \left(\varepsilon-\phi_{2}\right) \\
& :_{1}=0 \quad w_{1}-\left[p_{1} / q_{1}+b_{1}^{2}\left(q_{1} / p_{1}\right)\left(N_{2}-N_{1}\right)\right] \sin \iota_{1} \\
& +b_{1} b_{2} q_{2}\left(p_{3} / p_{1}\right) \cos \left(\varepsilon+c_{1}\right) \\
& \dot{q}_{2}=\omega_{c 2}-\omega_{2}-\left[p_{2} / q_{2}+b_{2}^{2}\left(q_{2} / p_{2}\right)\left(s_{3}-s_{2}\right)\right] \sin t_{2} \\
& -b_{1} b_{2} q_{1}\left(p_{3} / p_{2}\right) \cos \left(\varepsilon+b_{2}\right)
\end{aligned}
$$

The differences between the two levei and three lovel systems are confined to the polarization terms and to the phase angles coupling the polarization to the electric field

The equations thus far have no Insses and no pumping mechanism. We shall introduce these phenomenologically. Photons, wili be lost frem the cavity, so we damp the Electric field at a rate $B=1 / 2{ }^{\top} c$, wher $e^{~}{ }^{c} c$ is the cavicy $l$ ifetime. The principal contributions to this rate are transmission and absorption at the mirrors, and scattering and difraction losses. Each molecular ievel will be pumped at a rate $k$ and will gain or lose population through collisiunal processes (and spontaneous emission) at a rate $\gamma$. We will write this relaxation in a form appropriate to vibrational relaxation in that all relaxation will be to lowe energy states. This differs from the conmon practice or writing the relaxation so 
that the populations relax toward zoyo inversion (Pante11 and PuthoГt, 1969). The rate $y$ equals $1 / \mathrm{T}_{1}$, customarily called the "energy relaxation time". There is also a relaxation rate $\gamma^{\prime}$ for he polarization. In a semi-classical riew the polarization results when a photon is ausorbed and excites an oscillator, in phase with the exciting field. When the oscillators collide with other particles (or phonons in a solid) their phase is changed in a st.ochastic manner. The macroscopic polarization involves an average over all oscillators, thus it is large when the oscillators are all in phase and vanishes when the oscillators have random phase. The rate, $\gamma^{\prime}$, equals $1 / T_{2}$, the "phase relaxation time", and can be obtained from collisional line broadening data. When we insert the phenomenological damping rates we get equations of the form $\stackrel{\circ}{q}_{1}=-\beta q_{1}+p_{1} \cos \phi_{1}$ $\dot{p}_{1}=-\gamma_{1} p_{1}+b_{1}^{2} q_{1}\left(N_{2}-N_{1}\right) \cos \phi_{1}+b_{1} b_{2} q_{2} p_{3} \sin \left(5+\phi_{1}\right)$ $\stackrel{\circ}{N}_{1}=w_{1}+r_{2} N_{2}+2 p_{1} q_{1} \cos \phi_{1}$ $\stackrel{\mathrm{N}}{2}_{2}=\mathrm{w}_{2}+\mathrm{r}_{3} \mathrm{~N}_{3}-\mathrm{r}_{2} \mathrm{~N}_{2}+2 \mathrm{p}_{2} \mathrm{q}_{2} \cos \phi_{2}-2 \mathrm{p}_{1} \mathrm{q}_{1} \cos \phi_{1}$ ${\stackrel{\circ}{p_{3}}}_{3}=-r_{3}^{\prime} \mathrm{p}_{3}-\left(\mathrm{b}_{1} / \mathrm{b}_{2}\right) \mathrm{q}_{1} \mathrm{p}_{2} \sin \left(\varepsilon-\phi_{2}\right)$ $+\left(b_{2} / b_{1}\right) q_{2} p_{1} \sin \left(5-\phi_{1}\right)$ ${\stackrel{\circ}{q_{2}}}_{2}=-\beta q_{2}+p_{2} \cos \phi_{2}$ $\stackrel{\circ}{p}_{2}=-r_{2}^{\prime} p_{2}+b_{2}^{2} q_{2}\left(N_{3}-N_{2}\right) \cos \phi_{2}-b_{1} b_{2} q_{1} p_{3} \sin \left(\varepsilon+\phi_{2}\right)$ $\stackrel{\circ}{N}_{3}=w_{3}-r_{3} N_{3}-2 p_{2} q_{2} \cos \phi_{2}$ 
These equations, along with the $\delta_{k}$ and $\phi_{k}$ equations of 6.20 forth the complete set equations for the three-level laser. The next section examines their steady-state solution, as well as the conditions where $p_{3}$ can be neglected. Equations 6.21 reduce to a set of two-level laser equations coupled by a common level if $p_{3}$ vanishes.

\subsection{Steady State Values}

The equations for the two level system with relaxation terms are

$$
\begin{aligned}
& \stackrel{\circ}{q}=-\beta q+p \cos \phi \\
& \stackrel{\circ}{\mathbf{p}}=-\gamma^{\prime} p+b^{2} q\left(N_{2}-N_{1}\right) \cos \phi \\
& \dot{\circ}_{1}=w_{1}+r N_{2}+2 q p \cos \phi \\
& \dot{N}_{2}=w_{2}-r N_{2}-2 q p \cos \phi \\
& \dot{\phi} \div \omega-w_{c}-\left[p / q+b^{2}(q / p)\left(N_{2}-N_{1}\right)\right] \sin \phi \\
& \dot{\theta}=\Omega-w_{c}-(p / q) \sin \phi
\end{aligned}
$$

We can obtain a steady state solution for these equations by setting appropriate derivatives to zero. By setting $q$ to zero we obtain

$$
\mathrm{p}=\mathrm{Bq} \sec \phi \text {. }
$$

The populations, at least in a chemical laser, will. continue to rise, but the quantity $\stackrel{\mathrm{N}}{2}_{2} \stackrel{\circ}{1}_{1}$, will vanish at steady state. Substituting $\mathrm{Eq} \cdot 6.23$ into the difference

$$
\stackrel{\circ}{N}_{2}-\stackrel{\circ}{N}_{1}=w_{2}-W_{1}-2 \gamma N_{2}-4 p q \cos \phi=0
$$

gives

$$
4 \beta q^{2}=w_{2}-w_{1}-2 \gamma N_{2} .
$$


Multiplying the polarization squat ion by $q$ gives

$$
\begin{aligned}
\ddot{p} & =-\gamma^{\prime} q p+b^{2} q^{2}\left(N_{2}-N_{1}\right) \cos \phi \\
& =q^{2}\left[-\beta \gamma^{\prime}+b^{2}\left(N_{2}-N_{1}\right) \cos \phi\right] .
\end{aligned}
$$

which vanishes at steady state. This can occur only if $q=0$, implying no lasing, or if

$$
N_{2}-N_{1}=\left(B Y \cdot / b^{2}\right) \text { sec } \phi \text {. }
$$

Equations 6.25 and 6.27 describe steady state operation of a two-level laser. The inversion described by $\mathrm{Eq}: 6.27$ is the threshold inversion and the laser maintains itseif at that inversion. The threshold inversion gives the laser a gain which just equals the losses. Earlier computer models have assumed that the laser, if inverted, had an inversion which was constant at the threshold value. Equation 6.25 then represents energy conservation, because $2 \mathrm{~Bq}^{2}$ is the rate at which photons are removed from the cavity, and $w_{2}-w_{1}-2 \mathrm{YN}_{2}$ is the net rate of pumping the inversion. The additional factor of 2 multiplying $B q^{2}$ is because emission of one photon decreases the population difference by two molecules. There is also a transient period before the laser reaches steady state. We shall be concerned with that in the next section.

The steady state photon density, $q^{2}$, is expressed in terms of the pumping rate, $w_{2}$. This pumping rate will have a minimum value which will just achieve a threshold inversion. We shall now find this minimum value.

For simplicity assume that the pumping rates $w_{2}$ and $w_{1}$ are constant in time. Formally integrating the population equations of 6.22 we get 


$$
N_{2}-N_{1}=\left(w_{2}-w_{1}\right) t-2 \int_{0}^{t} r N_{2}\left(t^{\prime}\right) d t^{\prime} .
$$

Since we are interested in the time before lasing begins, $q_{I}=0$ and we have

$$
N_{2}=w_{2} t-2 \gamma \int_{0}^{t} N_{2}\left(t^{\prime}\right) d t^{\prime} \cdot
$$

These equations can be solved approximately by iteration, and for the first iteration we neglect the integral in Eq. 6.29 and substitute $\mathrm{N}_{2}\left(\mathrm{~L}^{\prime}\right)=W_{2} \mathrm{t}^{\cdot}$ in $\mathrm{Eq} \cdot 6.28$, giving

$$
N_{2}-N_{1}=\left(w_{2}-w_{1}\right) t-\gamma w_{2} \tau^{2} \text {. }
$$

This is quadratic in $t$, with the inversion negative for $t>\left(w_{2}-w_{1}\right) / \gamma w_{2}$ and maximum at $t=\left(w_{2}-w_{1}\right) / 2 \gamma w_{2}$. If we let the maximum inversion equal threshold, and neglect $W_{1}$, we get

$$
\left(N_{2}-N_{1}\right)_{t h}=\frac{B r}{b^{2}} \sec \phi=w_{2} / 2 r-w_{2} / 4 r
$$

which gives

$$
\mathrm{W}_{2}(\text { threshold })=4 \frac{\Delta Y Y}{b^{2}} \sec \phi .
$$

The approximations we have used would affect the numerical factor, 1 , but not the other parameters. In any case pumping at exactly $h_{2}$ (threshold) would not result in lasing because even if threshold is exceorlod it requires a finite time for lasing 10 build up. Therofore, the pump rate will have to exceed the threshold rate. 
We can normalize all pumping rates in terms of the threshold pumping rate. The pumping rates $h_{1}$ and $w_{2}$ have fixed ratios which depend on the pumping scheme, not the radiation parameters. As a result, we can write $w_{2}-w_{1}=k w_{2}$, where $k$ is a constant of the pumping, scheme. The expression for $\mathrm{q}^{2}$ also contains a term in the population, $\mathrm{N}_{2}$. We see by $\mathrm{Eq} \cdot 6.29$ that $\mathrm{N}_{2}$ is also a function of $\mathrm{w}_{2}$, so we can write $N_{2}=f_{2}(t) W_{2} t$, where $f_{2}(t)$ will depend on other parameters and will be of order unity. Using this we write

$$
\begin{aligned}
B q^{2} & =k W_{2} \sec \phi-1 / 2 r f_{2}(t) t W_{2} \\
& =\left(k \sec \phi-1 / 2 r t f_{2}(t)\right) \zeta \frac{4 B r r^{\prime}}{b^{2}}
\end{aligned}
$$

where $\zeta=w_{2} / w_{2}$ (threshold).

The angle variables $\phi$ and $\theta$ also have steady-s sate values.

$$
\tan \phi=\frac{\left(\omega-\omega_{C}\right)}{\left(B+\gamma^{\prime}\right)}
$$

and the actual laser frequency adjusts itself so that

$$
\dot{\theta}=0 \text {, }
$$

leaving 6 arbitrary. Any perturbation: such as noise, will make $\dot{\theta} \neq 0$ until the transient dies out. After the disturbance $\theta$ will have a new value, which is arbitrary since there is no restoring force about some preferred value. This completes the discussion of two-level steady state.

When we examine the steady state for the three level systen we must use Eqs, 6.21. The first gives us $B q_{1}=p_{1}$ cos $\phi_{1}$ in the steady state, and, similarly, $8 q_{2}=P_{2} \cos \phi_{2}$. Substituting thesr into the population 
equations and setting $\left(\dot{\mathrm{N}}_{2}-\dot{\mathrm{N}}_{1}\right)$ to zero, etc., after some algebra we get

$$
\begin{aligned}
& 2 p_{1} q_{1}=\frac{1}{3}\left(w_{3}+w_{2}-2 w_{1}\right)-r_{2} N_{2} \\
& 2 p_{2} q_{2}=\frac{1}{3}\left(2 w_{3}-w_{2}-w_{1}\right)-r_{3} N_{3}
\end{aligned}
$$

If we set $p_{3}=0$ and substitute in the equation for $\dot{p}_{1}$, we find, as in the two level case that

$$
\left(\mathrm{N}_{2}-\mathrm{N}_{1}\right)=\frac{\beta \gamma \dot{i}}{\mathrm{~b}_{1}{ }^{2}} \sec \phi_{1}
$$

The steady-state values of $q_{1}$ and $q_{2}$ are functions only of the pumping rates due to energy conservation as seen in Eq. 6.34. The polarizations, $p_{1}$ and $p_{2}$ are proportional to $q_{1}$ and $q_{2}$ at steady state, therefore, the only effect of $p_{3}$ on the steady state is a possible change in the threshold inversion. Let $\delta x$ be the perturbation in the inversion due to $p_{3}$ so that

$$
\mathrm{N}_{2}-\mathrm{N}_{1}=\frac{\beta \gamma_{1}}{\mathrm{~b}_{1}^{2}} \sec \phi_{1}+\delta \mathrm{n} \text {. }
$$

Substitute this in Eqs. 6.21 , setting, $\dot{\mathrm{p}}_{1}=0$ and $\dot{\mathrm{p}}_{3}=0$. The result is

$$
\delta n=\frac{\beta q_{2}^{2}}{\gamma_{3}^{1}}\left[\left(b_{2} / b_{1}\right)^{2} \sin ^{2}\left(\xi-\phi_{1}\right)-\sin \left(\xi-\phi_{1}\right) \sin \left(\xi-\phi_{2}\right)\right] \sec \phi_{1}
$$

As in the two level case we liun write $8 / 12^{2}$ as a lunction of $W_{2}$, which in turn is a function of other parameters. This Rives 


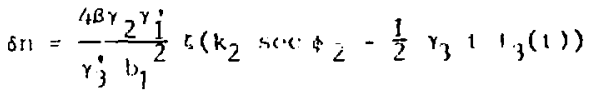

$$
\begin{aligned}
& x\left[+\frac{b_{2}^{2}}{b_{1}^{2}} \sin \left(\xi-\phi_{1}\right)-\sin \left(\xi-\phi_{2}\right)\right] \sin \left(\xi-\phi_{1}\right)
\end{aligned}
$$

where $k_{2}=\left(1 / k_{2}\right)\left(2 h_{3}-k_{2}-w_{1}\right)$ and $r_{3}(1)$ describes the dependence of $\mathrm{N}_{3}$ on $\mathrm{k}_{2}$. Both $\mathrm{k}_{2}$ and $\mathrm{i}_{3}$ are of order unity. If we divide in: the two level threshold value: of $\left(\mathrm{N}_{2}-\mathrm{N}_{1}\right)$ we get

$$
\begin{aligned}
\frac{\delta n}{\left(N_{2}-N_{1} J_{t h}\right.}= & \frac{4 Y_{2}}{Y_{3}^{\prime}}\left[k_{2} \sec \phi_{2}-\frac{1}{2} r_{3} t f_{3}(t)\right] \\
& \times\left[\left(b_{2} / b_{1}\right)^{2} \sin \left(\xi-\phi_{1}\right)-\sin \left(\xi-\phi_{2}\right)\right] \sin \left(\xi-\phi_{1}\right)
\end{aligned}
$$

Thus the net effect of the coupling, $p_{3}$, is to increase the inversion between levols 2 and 1 , since $\left(b_{2} / b_{1}\right)>1$ for a harmonic oscillator. A similar treatment for the inversion between $\mathrm{N}_{3}$ and $\mathrm{N}_{2}$ would show a decrease.

Becanse $r_{3}$ is usually several orders of magnitude larger than $r_{2}$ the effect will be very small unless the pumping, represented by $\zeta$ is very 1arge. In the case of the chemically pumper laser 5 may be large enough to make on an appreciab]s quantity.

1t appeurs then that in the steady state case the coupling will not be important. The principal effect is to cause the laser to operate at an inversion somewhat different than the threshold inversion for : pair of two level lasers using a common level. The perturbation, $b n$, on the inversion 
is small when the pumping is just above threshold, so has a minor effect in that case. When the laser is strongly pumped on becomes appreciable but is still orders of magnitude smaller than actual populations, so it will have little effect on the laser output. Therefore, we conclude that we can neglect $p_{3}$ in the steady state.

The fact that we have a steady state solution with $p_{3}$ included doesn't guarantee that the laser will operate at the steady state; the steady state may represent an unstable solution. Stability will be examined in a qualitative fashion. Assume that $q_{2}$ rises a bit above its steady state value. Examination of Eqs, 6.21 shows that this will cause $p_{3}$ tc increase, which in turn causes $p_{1}$ and $q_{1}$ to increase. However, the sum $q_{1}^{2}+q_{2}^{2}$ is basically determined by energy conservation, so both cannot remain above steady state for long. A similar argument holds if $q_{1}$ is increased. This shows that $\mathrm{p}_{3}$ cannot rob power from one lasing transition and feed it to the other. The argument is not sufficient to show that $p_{3}$ will not cause an oscillation centered about the steady state. Thus far we have neglected the effect of $\sin (\zeta-1)$ on $\mathrm{p}_{3}$. The angle $\zeta$ is the sum of three arbitrary phase angles $\theta_{1}, \theta_{2}$, and $\theta_{3}$. They may assume any value at steady state and will change value upon any fluctuation of the other variables. As a result, the value of $\sin \left(5-p_{1}\right)$ will be stochastic and will have an average value of zero when averaged over some time, unless something locks $\theta_{1}, \theta_{2}$, and $\theta_{3}$ 
to some fixed value. This can occur under conditions such as those that cause mode locking, so that we can assume that the transient effects of $p_{3}$ will be associated with rapid variations like mode locking. Since we have already decided to neglect mode locking, we will also neglect $p_{3}$ and treat the cascade as a set of two-level transitions coupled only by sharing a common level. From here on we will consider only cascades with the radiation in each transition treated by equations for a two-level laser.

\subsection{Stability of Two Level Laser Equations}

The semi-classical equations of motion for a two level laser, including phenomenological damping terms, are

$$
\begin{aligned}
q & =-B q+p \cos \\
\dot{p} & =-r^{\prime} p+b^{2} q\left(N_{2}-N_{1}\right) \cos \\
\mathbb{N}_{1} & =H_{1}+r_{2} N_{2}+2 p q \cos \\
\mathbb{N}_{2} & =H_{2}-r_{2} N_{2}-2 p q \cos \\
: & =\Omega-\omega_{c}-(p / q) \sin \\
: & =\omega-\omega_{c}-\left[p / q+b^{2}(q / p)\left(N_{2}-N_{1}\right)\right] \sin
\end{aligned}
$$

where $q^{2}$ is the density of photons in the cavity, and $p$ is proportional to th polarization. The angle is the phase angle between $q$ and $\dot{p}$, and $\theta$ is an arbitrary phase angle. The constant $b$ is proportional to the dipole matrix element. There are three frequencies in these equations: $a$, the molecular resonance frequency, ${ }^{\prime} c$ ' the cavity resonance frequency, and $w$, the laser frequency, which 1 les between 
$n$ and $\omega_{c}$. If the cavity resonance, $\omega_{c}$ ' is tuned exactly to the molecular resonance $\Omega$, then $\Omega=\omega_{c}=\omega$. Because the cavity is many wavelengths long, the cavity will have many resonance frequencies, $\omega_{c}$, spaced $c / 2 L$ apart. For any value of $\Omega$ there will be a cavity resonance, $\omega_{c}$ ' such that $\left|\Omega-\omega_{c}\right| \leq c / 4 \mathrm{l}$. If $\Omega=\omega_{c}$, Eq. $6.40 f$ shows that $\$$ will be driven to its steady-state value of zero. The laser is then said to be on tune. When $\phi=0$ the cosine terms are unity and we can neglect Eqs. 6.40e and 6.40r. The angle $\theta$ will be constant and we will neglect it because we are not studying temporal coherence.

\subsubsection{Laser Rate Equations}

Before considering the generai case we will consider a speciai case which gives considerable insight. Solid state lasers are generally operated under conditions where $r^{\prime}$ is the orders of magnitude faster than other rates. Gas lasers operated at pressures of several Torr are generally ccllision broadened and also have $r$ the fastest rate. In that rase, if there is a perturbation from steady-state $\dot{p}$ will return to zero before the other variables. Setting $\stackrel{\circ}{\mathrm{p}}=0$ we get

$$
p=\left(b^{2} q / r^{\prime}\right)\left(N_{2}-N_{1}\right) \text {. }
$$

Let $\psi=q^{2}$, the photon densjty, and substitute in Eqs. 6,40. The result is the well known laser rate equations,

$$
\begin{aligned}
\psi & =-2 B \psi+a \psi\left(N_{2}-N_{1}\right) \\
\left(\stackrel{\circ}{N}_{2}-\stackrel{\circ}{N}_{1}\right) & =w_{2}-w_{1}-2 \gamma N_{2}-2 a \psi\left(N_{2}-N_{1}\right) .
\end{aligned}
$$


These equations give a reasonably good description of the major reatures of laser behavior, especially in the regime $\gamma \gg \beta, \gamma$. The parameter a equals $2 b^{2} / \gamma^{\prime}$. If we write $\left(N_{2}-N_{1}\right)=\Delta N$ we get steady state solutions

$$
\begin{aligned}
\Delta N & =2 \beta / a=\theta \gamma^{\prime} / b^{2} \\
4 \beta \psi_{0} & =W_{2}-W_{1}-2 \gamma N_{2}
\end{aligned}
$$

For algebraic simplicity we will assume $W_{1}=0$. If $W_{1}$ were not zero, the answer would differ by numerical factors of order unity. The value of $\mathrm{N}_{2}$ is obtained from

$$
\begin{aligned}
& N_{2}+N_{1}=H_{2} t \\
& N_{2}-N_{1}=B_{Y} / b^{2}
\end{aligned}
$$

which gives

$$
N_{2}=1 / 2 W_{2} t+B \gamma^{\prime} / 2 b^{2} .
$$

The maximun inversion, in the absence of lasing occurs at $t=1 / 2 \mathrm{r}$, so we will anaiyze stability at that point in time, giving

$$
\mathrm{N}_{2}=w_{2} / 4 Y+e y^{\prime} / 2 b^{2} \text {. }
$$

The pump rate, $w_{2}, w_{i} \&$ defined in Eq. 6.31

$$
W_{2}=45 \frac{B r r}{b^{2}}
$$

where $5=w_{2} / w_{2}$ threshold. This gives

$$
N_{2}=(5+1 / 2) \frac{B y^{\prime}}{b^{2}}
$$


Substituting in Eqs. 6.43 we eet the steady-state solutions

$$
\begin{aligned}
\Delta N_{0} & =\beta_{\gamma} / b^{2} \\
\psi_{0} & =(s-1 / 2) r r^{\prime} / 2 b^{2}
\end{aligned}
$$

To investigate the stability we linearize Eqs. 6.42 about the steady-state value by writing

$$
\begin{aligned}
\Delta N & =B \gamma^{\prime} / b^{2}+\delta N \\
\psi & =\psi_{0}+\delta \psi,
\end{aligned}
$$

and substituting. We neglect terms in the product $\delta \psi \delta \mathrm{N}$, and find that the steady state terms cancel. The resulting linearized equations are

$$
\begin{aligned}
& \delta \dot{q}=\left(\xi-\frac{1}{2}\right) \gamma \delta \mathrm{N} \\
& \delta \stackrel{0}{\mathrm{~N}}=-48 \delta \psi-2 \mathrm{r} 5 \delta \mathrm{N} .
\end{aligned}
$$

These can be converted to a single second order differential equation by differentiating the first equation and eliminating $\delta \dot{N}$ and $\delta N$. The result is

$$
\delta \dot{\delta}+2 \zeta \gamma \delta \dot{\psi}+4\left(\zeta-\frac{1}{2}\right) \text { बr } \delta \psi=0
$$

Letting $\delta \psi=e^{\lambda t}$ gives the characteristic equation

$$
\lambda^{2}+25 \gamma \lambda+4\left(c-\frac{1}{2}\right) \text { Br }=0
$$

which has roots

$$
\lambda=\nu+i \nu=-5 \gamma \pm i \sqrt{4 \beta \gamma(\zeta-1 / 2)-\zeta^{2} y^{2}}
$$

Thus the serturbation will be a damped oscillation at a frequency v (radians/scr) about the steady-state value in the region where $s>1 / 2$ and $4 \beta \gamma(s-1 / 2)>r^{2} s^{2}$. 
Contour plots of the treguency, v, and dampine? constant, $u$, are shown in lig. 6.2 as a linct $i$ on of $Y$ and $B$. In these plots the ratio $y^{\prime} / Y=10^{4}$. It is reasonable to keep this ratio constant since both relaxation rates are proportional to the molecular collision probability provided $\gamma$ is fast compared to spontaneous emission. The ratio, $10^{4}$, is the right order of magnitude for HF. For most other molecules the ratio would probably be much larger since vibrational relaxation of $\mathrm{HF}$ is anomalously fast. In the contour plot the pump rate is held constant for all values of $B$ and $r^{\prime}$ so that the pump parameter, $\zeta$, varies on the graph. Contours of constant $\zeta$ are shown on the graphs as straight lines with a slope of $-1 / 2$ on the logarithmic plot. For values of $\zeta<3$ the laser is below threshold, anu for $\zeta<1 / 2$ one of the two real roots is positive, and the irequency is zero. For moderate values of $5 y$ the solution oscillates and for large values of $\zeta \gamma$ the solution is overdamped and again has two real roots. The more positive of these roots is shown on the contour plot since this represents the slowest recovery from the transient. As a result, the rastest time constarts occur on the boundary between oscillating and overdamped solutions. This boundary is a straight line with a slope of -2 . Values of $B$ and $r^{\prime}$ to the left of the boundary yield overdamped solutions.

The variables $\gamma^{\prime}$ and $B$ represent parameters which are readily adjustable experimentally. The photon decay rate, $2 \mathrm{~B}$, can be adjusted by changing mirror reflectivities or the 
cavity length. The collision broadenirg rate, $r^{\prime}$, is adjustable by changing the pressure of the gas, or by adding other species. The ratio $\gamma \% / \gamma$ depends on molecular parameters and can be changed only a small amount by adding other species. The pump rate is also experimentally adjustable; the contour plot for other pump rates looks the same except for translation roughly perpendicular to lines of constant $b$. The translation is obvious from an examination of Eq. 6.50.

Laser rate equation: (6.42) have no undamped oscillating solution because the real part of $h$ is alway negative. This result has previcusly been noted by Makhov (1962) and Sinnet (1962). Even in the case that $4 k^{2} \zeta^{2} \gamma^{2}>8 B k \zeta \gamma$ the real part of $\lambda$ is negative. As we shall see below, this is not always true if we do not force $\dot{p}$ in Eq. $6.42 \mathrm{~b}$ co vanish. When $\zeta$ is reasonably small, e.g., 2 to 10 , the solution will be an oscillation with frequency on the order of $\sqrt{\mathrm{Br} \zeta}$ damped at a rate $\gamma$. Since $\beta \gg \gamma$ under common laser operating conditions, the oscillation will continue for possibly tens or hundreds of cycles before it is damped to negligible proportions. As the umping parameter, $\zeta$, is increased both the frequency and the damping rate increase, but the damping rate increases much faster. As a result, if the laser is pumped harder it is danped rapidly, but a weakly pumped laser will oscillate for a long time. If the laser is pumped hard enough the damping will eventually be so fast that no oscillatjon at all will occur. 
6.4.2 Semi-classical Equations with Cavity Tuned to Atomic Resonance

he will consider next the case where the laser is on tune, i.e. $\Omega=\omega=\omega_{c}$. In that case it is easily seen from $E q .6 .4 \mathrm{Cf}$ that the steady state value of $\phi$ is 0 or $\pi$ and $\cos \Phi= \pm 1$. Further examination shows that the solution $\phi=0$ is stable when the inversion, $N_{2}-N_{1}$, is positive, and that $\phi=\pi$ is stable when the inversion is negative. Steady state lasing occurs only for a positive inversion, so when the laser is on tune we get the following equations.

$$
\begin{aligned}
\dot{q} & =-\beta \mathrm{q}+\mathrm{p} \\
\dot{\mathrm{p}} & =-\mathrm{r}^{\prime} \mathrm{p}+\mathrm{b}^{2} \mathrm{q}\left(\mathrm{N}_{2}-\mathrm{N}_{1}\right) \\
\Delta N & =N_{2}-N_{1}=w_{2}-w_{1}-2 \gamma N_{2}-4 p q
\end{aligned}
$$

The steady state solution for these equations is

$$
\begin{aligned}
& q_{0}^{2}=1 / 4\left(h_{2}-w_{1}\right)-1 / 2 \gamma N_{2}=(2 b-1) r r^{\prime} / 4 b^{2} \\
& p_{0}=B q \\
& \Delta N_{0}=B \gamma^{\prime} / b^{2} .
\end{aligned}
$$

As before we linearize by setting

$$
\begin{aligned}
\mathrm{q} & =\mathrm{q}_{\mathrm{o}}+\delta \mathrm{q} \\
\mathrm{p} & =\mathrm{p}_{\mathrm{o}}+\delta \mathrm{p} \\
\Delta \mathrm{N} & =\mathrm{N}_{\mathrm{o}}+\delta \mathrm{N}, \\
\mathrm{N}_{2} & =B \gamma \cdot / \mathrm{b}^{2}+1 / 2 \delta \mathrm{N}
\end{aligned}
$$


and neglecting products of the form $\delta q \delta N$, etc., we obtain the linearized equations

$$
\begin{aligned}
& \delta \dot{\mathrm{q}}=-\beta \delta \mathrm{q}+\delta \mathrm{p} \\
& \delta \dot{\mathrm{p}}=-\gamma^{\prime} \delta \mathrm{p}+\beta \gamma^{\prime} \delta \mathrm{q}+\mathrm{b}^{2} \mathrm{q}_{0} \delta \mathrm{N} \\
& \delta \dot{\mathrm{N}}=-\gamma \delta \mathrm{N}-4 \mathrm{q}_{0}(\beta \delta \mathrm{q}+\delta \mathrm{p}) .
\end{aligned}
$$

Substituting $q=e^{\lambda t}$ and differentiating, we obtain the characteristic equation

$\lambda^{3}+\left(\beta+Y+\gamma^{\prime}\right) \lambda^{2}+\left(\gamma \beta+2 \zeta \gamma^{\prime}\right) \lambda+2(2 \zeta-1) \beta \gamma \gamma^{\prime}=0(0.55)$

This characteristic equation is the same as Shirley (1968), even though we treated the relaxation of population somewhat differently. Analytic expressions for the roots of this equation exist, (Birkboff and MacLane, 1965), but the algebraic expressions are too complicated to be of much use.

Because the equation is cubic it must have either one or three real roots. Because all the coefficients are positive, all of the real roots must be negative (Turnbul1, 1957). Is there is only one real root there will be two complex conjugate roots and the perturbation will be

$$
\delta q=c_{0} e^{(\mu \pm i v) t}=c_{0} e^{\mu t} \cos (v t+n)
$$

where $c_{0}$ and $n$ are constant or integration. The steady state solutjon'will be a stable solution it and only if $\mu$ is negative. If $\mu$ is positive the output will oscillate about the steady with an exponentially increasing magnitude. (The exponentially growing solution is a small perturbation approximation; in roality tho nonlinear terms will cause the 
amplitude to assume a finite upper limit. This corresponds to ererky conservation.) We wish to find the domains in which the steady-state solution is stable or mstable. The roots of Eq. 6.55 , the characteristic stability equation for the seini-classical laser on lune, are shown in Fig. 6.3. The damping constant is the most positive real portion of the three roots. The upper left-hand corner is almost identical to the plot for the rate equations, which is expected since this is the region where $Y^{\prime} \gg B$, the assumption used to obtain the rate equations. In the lower right hand corner the oscillation is unstable, in contrast to the solution for the rate equations. Also, the solution is oscillatory for all values of $Y^{\prime}$ and $B$ if $\delta>10^{3}$. In the case of rate equations the contours for damping constant were straight lines with slopes of about -1 . In the case of the semi-classical equations these contours are folded back in the lower right hand corner. As the pumping rate is changed the plot translates in the same manner as Fig. 6.2. The behavior of the laser in the various regions of Fig. 6.3 is illustrated by numerically integrating equations 6.51 . The resulting photon itensities in the cavity are plotted as a function of time in $\mathrm{Fig} .6 .4$. In the unstable region, curve $A$ in Fig. 6.4 and point (A) in Fig. 6.3, the solution in a series of spikes. In the small signal approximation of Eq. 6.55 the envelope of the spikes should grow exponentially in time. In the numerical soiution the nonlinear nature of the equations causes saturation eflects which 
limit the amplitude of the spikes. Physically, this is a result of energy conservation. In fact, in the numerical solution the first spike is the largest and it is rapidly damped. The first spike ss large because there is a large delay to turn-on and a great deal of energy is stored in the inversion. After the first few spikes the laser settles down to a steady oscillation with an average power output equal to the steady state output. The peak power of each spike is many times the average power, but the duration of the spike is short compared to the time between spikes. A plot of the corresponding inversion shows the sawtooth shape characteristic of relaxation oscillations.

There are about 17 spikes per microsecond correponding to an angular frequency of $1.2 \times 10^{8} \mathrm{radian} / \mathrm{sec}$. Equation 6.55 gives a frequency of $1.4 \times 10^{8}$ at point (A). Figure $6.4 \mathrm{~b}$ shows the time history of the photon density in the overdamped region at point $B$. There is no oscillation, in agreement with Fig. 6.3; and the damping constant appears to be close to the 40 nsec. calculated from Eq. 6.55. As expected, a similar result was obtained when \$ was allowed to vary.

Point (C) represents the condition where the photon density is a tamped oscillation. The frequency, $3.2 \times 10^{7} \mathrm{rad} / \mathrm{sec}$, agrees with the value frum Eq. 6.55; and the damping constant appears to equal the predicted value of 100 nsec. 


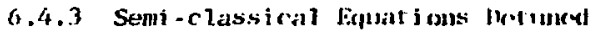

The stability af Eqs. 6.40 can also lo detcomilled for the case where thr laser is chltuted. In thal cousc tine steady silatr solution is

$$
\begin{aligned}
q_{0}^{2} & =(2 b-1) r r^{\prime} / \mu_{1} b^{2} \\
\nu_{0} & =q_{0} i \cos \omega_{0} \\
A N_{0} & =\frac{B r^{\prime}}{b^{2} \cos ^{2} \omega_{0}} \\
\tan \phi_{0} & =\frac{A \omega}{B+r^{\circ}}=T
\end{aligned}
$$

Again we 1 inearize using Eq. 6.53 and

$$
\phi=\phi_{0}+\delta \phi i
$$

and use the approxinations : $\cos \delta \theta=1, \sin \delta \phi=\delta \phi$. When this is substimited in Eqs. 6.100 we obtain the following equation, written in matrix form.

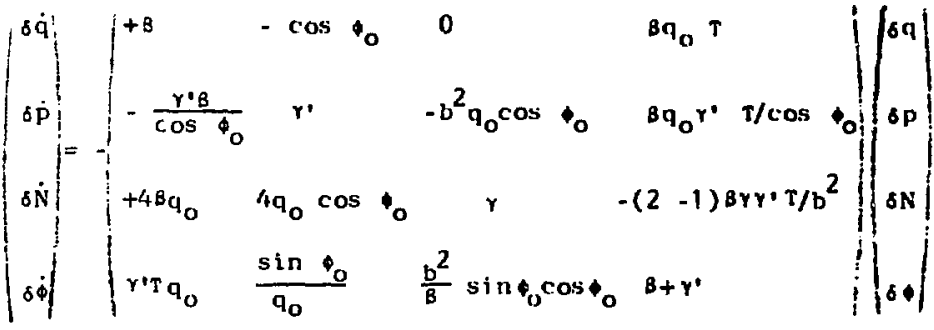

$$
\begin{aligned}
& \text { (6.58) }
\end{aligned}
$$

Designating the matrix as $A$ and the vector $y$ this can be written as

$$
\stackrel{0}{y}=-A y
$$


with the formal solution

$$
y=y_{0} e^{-A t} \text {. }
$$

which is well defired, bus difiticult to evaluate. Stace the rquation is litwal we can iry a culution of the form y - cte where $c$ is a veet or of constint co-effictents, and $a$ is a

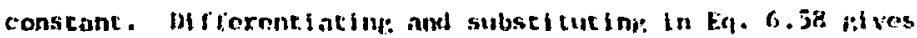

$$
\operatorname{Aco}{ }^{\lambda t}=-A \cos ^{\lambda t}
$$

Mividing by $\operatorname{crc}^{\lambda t}$ fivess

$$
\lambda \cdot \lambda=0
$$

where I ts the imb diapomal inatrix. Exaluating thr detorminant of Eq. G.60 fives the charactertstic cquat ion whith we used previonisly to find the stablity conditgons. Equntion 6.60 also defines the elgenvalites of thr matrix $n$. Therefori: rather thall evaliner the determinant, the eigenvalues of $A$ woro olstilned numrically. The eigenvalues can be elcher real or complex conjugnte pairs. The laser paraneters for figs. 6.2 and 6.3 were used with a sw of $7 \times 10^{8}$ radians/sec (approximately the maximur detunini, for a cavity wtih a round trip thase of $5 \mathrm{~ns}$.$) : there were at inst$ one complex conjulgate pair of eigenvalues and pither two or lour real eigenvalues. A contour plot of the most positive real part of any of the cigrnvalues is shown in tig. 6.5a, and a contour plot of the frequeney $i$.. shown in tip. $3.5 \mathrm{~b}$. Comparison with ligs. 6.2 and 6.3 shows that the principal effect of including, detuning, is to cularge the domain of instability. There is a repion within the unstable donain 
which is also noll-oscillatory. This represonts a growing "xponventia? altput. As noterel bersus, this growth is wentually limited by nomlineitr torms in rhe original cruations $(6.40)$. The larpest positive eipenvalue $\left(.4 \times 10^{8}\right)$ ocruys nr:at point. (A).

The effect of allowing to vary with parameters for point (A) is seen by comparing Fig. 6.6a with Fig. 6.4a. The variation of the phase angle tends to damp the oscillations. This is in approximate agreement with the eigenvalues pottod in lig. 6.5. The frequency is zero, and the calculated wavelorms have litt]e: oscillation.

In general, allowinf to vary did not result in vastly different waveforms. However it required several I iturs as much computer time.

\section{5 simmary}

We have found that we can accurately estimate the stistily of the laser abut its steady state value by studying, the lineari\%ed response to a small perturbation about the steady state value. In contrast to the stability criterion obtained from laser rate equations, there is an unstable region when $B \geq Y^{\prime}$, provided the pumping parameter $\delta$ i.s large. In the region $\theta \leq .1 Y^{\prime}$ the stability is accurately predicted from laser rate equations, provided $\zeta$ i.s not too large, e.g. $\zeta<10^{3}$. Most lasers are operated with high reflectivity mirrors which make $B$ relatively small. Since it is usually difficult to pump a laser, most lasers

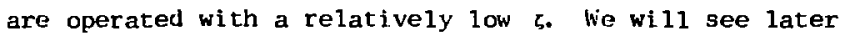


that an HF chenical laser i.s very strongly pumped, and because of its high gajn can be operated with low reflectivity mirrors, resulting in a larpe B. This is likely to cause unstable operation of the laser, or a damping constant which is large compared to the pulsewidth. 


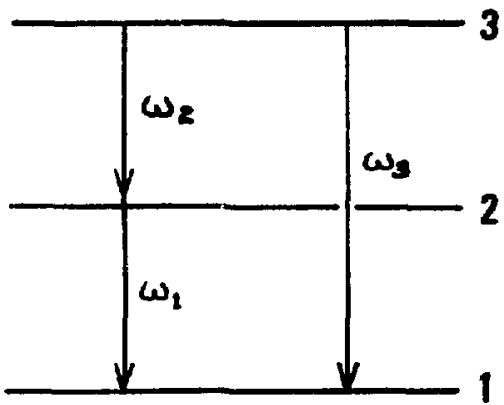

Fig. 6.1 Levels and transitions for at rhree: level cascade. 


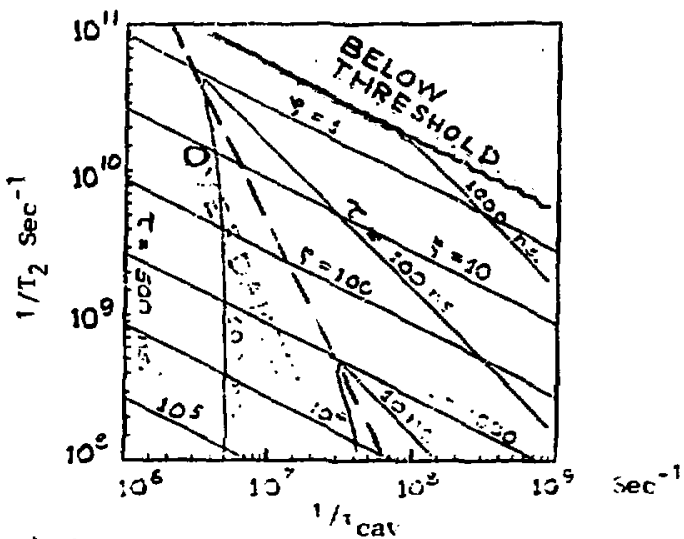

a) isamping co-elricient - 1

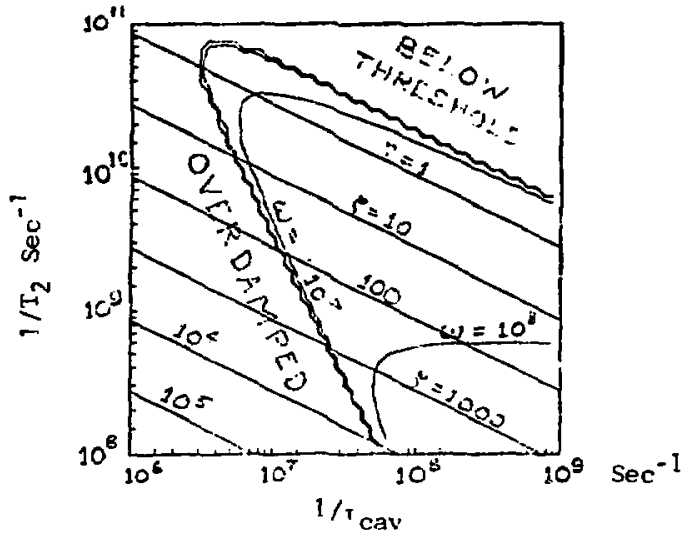

b) Frequency of osciliation - w

Fig. 6.2 Contour plots of damping co-efricient and frequency of oscillation I rom laser rate equations ( 6.42$)$. Enerfy relaxation $t$ ime $r_{1}$ is $10^{4} r_{2}$. The parameter 5 is defined in the text. 


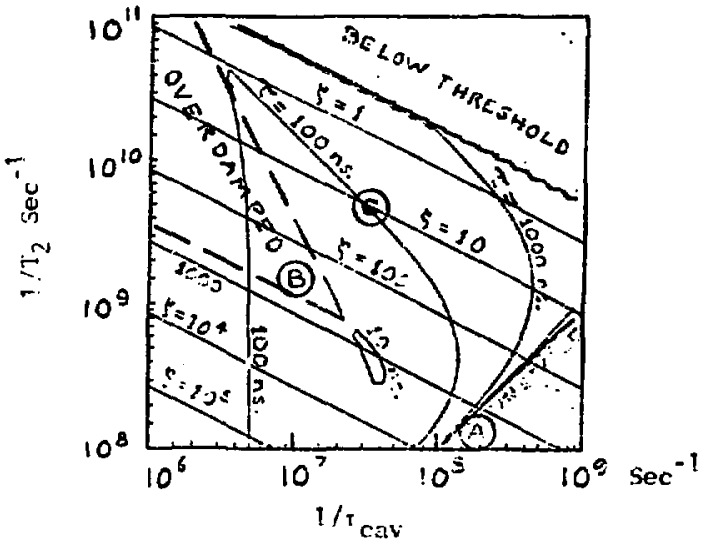

a) jasping co-efficient - T

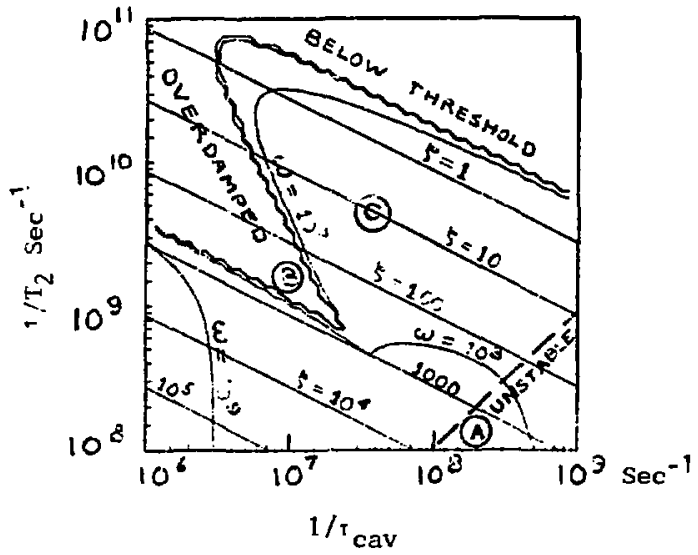

b) Frequency of oscillation - $\omega$

Eig. 6.3 Contour plots of damping co-efficient and frequency of oscillation for Eq. 6.55. Parameters are the same as Fig. 6.2. 


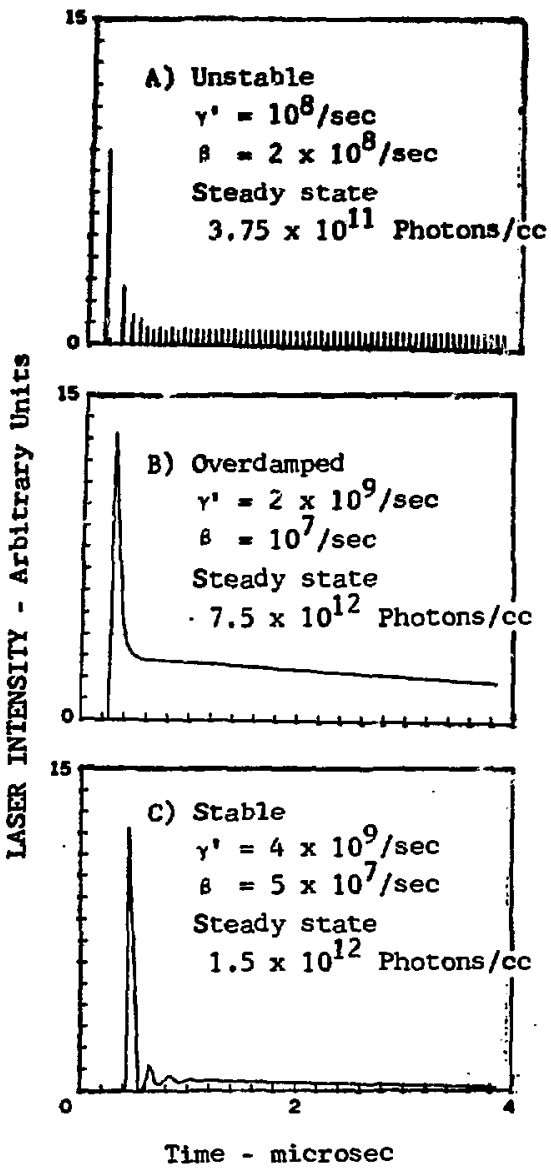

73. 6.4 Laser waveforms calculated from semi-classical equations (6.51) with cavity tuned exactly to atomic resonance. 


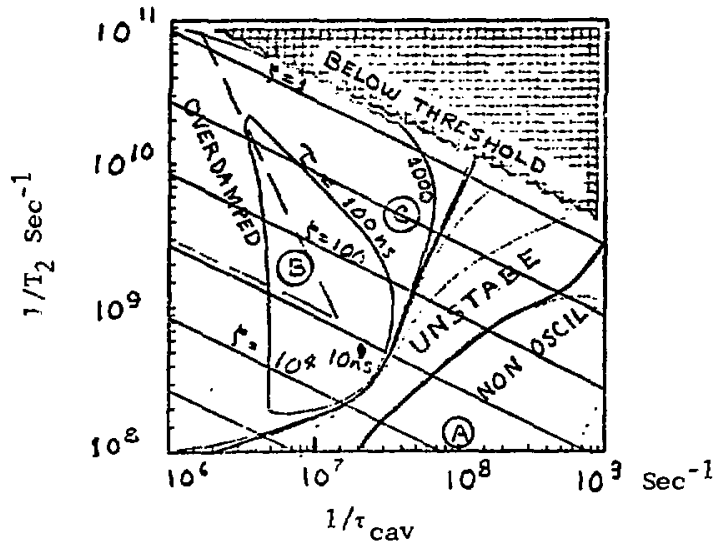

a) Most positive of 2 damping co-efficients - I

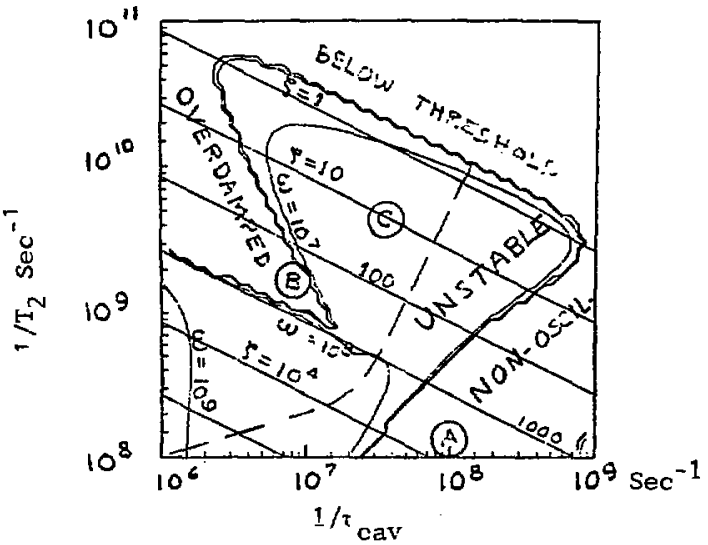

b) Frequency of oscillation

Fig. 6.5 Contour plots of damping co-efficient and frequency of oscillation for Eq. 6.58. There were either 2 or 4 real roots. Figure $6.5 a$ shows the value of the least stable root. Parameters are the same as Fig. 6.2. 

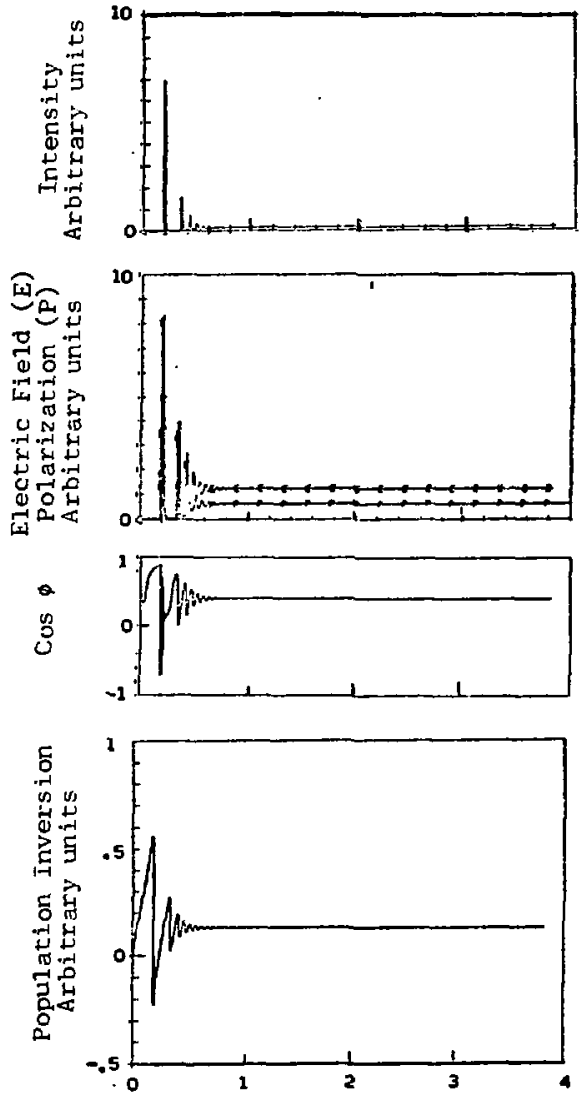

Time-microseconds

Fig. 6.6 Laser waveforms calculated using semi-classical equations ( 5.40$)$ with laser detuned $7 \times 10^{8} \mathrm{rad} / \mathrm{sec}$ and phase angle, $\phi$, allowed to vary. Other parameters are the same as Fig. 6.4A. 
7. THE TXTON $^{\dagger}$ COMPUTER COUE

\subsection{Introduction}

The laser equations discussed in the previous section form the basis for a model of the HF chemical laser which treats each rotational level separately. Changes are required in the population equation: the relaxation term must be modified to include relaxation to adjacent rotational states, and most states are coupled to a higher vibrational state as well as a lower one. There are no formal mathematical problems involved in doing this because the damping term was introduced phenomenologically and can be altered on the same basis. he have already shown that a cascade can be treated as a series of two-level transitions coupled only by a common population.

A simplified set of semi-classical laser equations was chosen. The simplification consists of calculating the steady-state value of the phase angle, $\phi,\left(E_{q}, 6.40\right)$, and holding constant throughout the calculation. Tests showed that integrating the differential equation for increased the computing time an order of magnitude. The only major difference in the results was reduced spiking amplitude when $\$$ was allowed to vary. It was decided to hold fixed in order to stay within the allocared computer budget. Laser rate equations would require about the same computer time as

\footnotetext{
Ixion was a demi-god from Greek mythology. He murdered his father-in-law and became insane. Finally Zeus purified him so that he could live on Mt. Olympus. He repaid this kindness by at tempting to seduce Hera, Zeus's wife. Ixion was tricked into a uni on with a cloud, rather than Hera, and thus begat the Centaurs. For his treachery zeus sentenced him to be bound to a wheel and rotate forever in the air. This clearly is classical rotational motiori without relaxation.
} 
the simplified semi-classical equations. Rate equations assume that $\dot{p}$ is always small, but under some conditions in the HF laser th:s may not be true. The simplified semiclassical laser equations give more accurate results so they were used in the IXION code with modified relaxation terms. The IXION code consists of a main program and a number of subroutines. The main code sets the initial conditions, and then calls the GEAR package of subroutines at each time step. After every few time steps it writes the current values of the populations and laser intensities into a disc file named SCRATCH. When the calculation has reached a preset time the main program terminates the problem.

The GEAR package consists of subroutine STIFFB to integrate the differential equations and associated subroutines for doing the associated matrix algebra. The GEAR package contains a method for integrating stiff differential equations or an alternate Adams predictor-corrector scheme. A sentinel can be set to select either method. Initíal calculations showed that the equations were not stiff, but required short time steps because of the oscillatory nature of the solutions. Iests showed that the time step with the stiff method was twice as large as the time step with the predictor-corrector method. However, the stiff method required about five times as much computer time due to inverting, the Jacobian matrix. The predictor-correctol method was chosen for most calculations because il was murh lasi.r duc to not having to invert the 
Jacobiatl inalrix. The GEAR paskils: obtatined the appropriats differenrial equations from subrout ine bllfFUNB.

Subroutine DJFFUNB furnishos the current numerical value of each time derivative $l o$ the GEAR package on each predictor or corrector step. There is a time derivative for each population level and two derivatives for each laser transition, one each for the electric field and the polarization. The code was dimensioned to treat six vibrational levels and up to 20 rotational states in each level. When the laser equations are added there are nearly 300 variables requiring Eimg derivatives. Fortunately, there are only three types of variables so the subroutine has only three differential equations with indices corresponding to the appropriate quantum numbers. These equations will be described in detail. DIFFUNB requires certain rate constants which it obtains by calling subroutine CHEMIST. DIFFUNB also calculates equilibrium constant from spectroscopic data.

Subroutine CHEMIST calculates the rate of formation of $\mathrm{HF}$, the basic rate constants for vibrational and rotational relaxation and iine broadening. All of these are proportional to collision frequenciea, and therefore are proportional to the densities of various chemical species. Subroutine CHEMIST contains the appropriate chemical kinetics equations. FORTRAN listings of subroutines DIFFUNB, CHEMIST can be found in Appendix $\mathrm{F}$.

The output of the IXICN code is contained in a disc file named SCRATCH. This is organized in blocks of about 200 variables (populations and intensities) at each time. 
The file is written in $\mathrm{BCD}$ format rather than binary so that it can be read by humans as well as computers. In order to obtain time histories and plots, a separate computer code, EDITB, was written. This code, described below, reads SCRATCH, sorts the data into time histories and gives both graphic and tabular output. The results of the calculations will mainly be presented in the form of these graphs.

\subsection{Ordering of the Molecular States}

Computer solution of the equations requires that the states be ordered in some fashion. A spectroscopist would be inclined to order the states by quantum numbers, since this would order states in terms of energy. However, in considering a laser it is natural to order the states in cascades. A cascade is the set of level.s which are connected by a series of radiative transitions (states coupled by diagonal lines in Figure 7.1).

When the computer output is organized in cascades the physical significance of the cascades is seen immediately because it is easy to see inversions. If the radiation is also ordered by cascades it is easy to see how the transitions interact via the common levels.

The cascade also turns out to be the natural ordering for numerical purposes. If the Gear method is used to integrate the differential equations the variables must be organized into a vector $y$ with a derivative vector $\dot{y}$. The corrector step requires iteration by the Newton-Raphson 
technique and this requires inversion of the Jacobian matrix $\left(\partial \dot{y}_{i} / \partial y_{i}\right)$. The inversion is greatly simplified if the nonzero matrix elements are concentrated in a narrow band about the diagona1. The narrowest bandwidth is achieved when the molecular levels are organized as a cascade, and the differential equations for radiative transitions are placed between those for the two levels connected by the transition. The equations have been ordered in this way in the IXION code. For example, if $\mathrm{Y}(\mathrm{M})$ is a population. $\mathrm{Y}(\mathrm{M}+1)$ is a polarization, $Y(M+2)$ is an electric field and $Y(M+3)$ is the population of the level coupled to $\mathrm{Y}(\mathrm{M})$ by the transition $\mathrm{Y}(\mathrm{M}+2) * \mathrm{Y}(\mathrm{M}+1)$.

\subsection{The Population Equation}

The basic population equation is

$$
\dot{\mathrm{N}}_{1}=\mathrm{w}_{1}+\mathrm{r}_{2} \mathrm{~N}_{2}+2 \mathrm{pq} \cos \bullet \text {. }
$$

The first two terms were introduced phenomenological1y, so it is equally proper to rewrite them to correspond to the scheme of Fig. 7.1. The IXION code uses the following population equation (in FORTRAN notation).

$$
\operatorname{YDOT}(M)=2 . * C P H *(A V 6 * Y(M+1) * Y(M+2)-A V 1 * Y(M-2) \div Y(M-1))
$$

A

B

C

D

E + DETAIL $(I V, J) * H F D O T$ $+V D O T *(V P * B N(J) * Y(M+3)-V * B N(J+1) * Y(M))$ $+R D O T *(Y(M+16) * A J * D J(J+1))$

$$
-(E Q K(I V, J) * D J(J+1)+D J(J)) * Y(M)
$$$$
+E Q K(I V, J-1) * Y(M-16) * D J(J)
$$ 
The first line represents the interaction with the radiation, Line $A$ is the chemical source term. Line $B$ is the vibrational relaxation term and the remaining lines are rotational relaxation terms. These will be discussed in detail.

\subsubsection{The Chemical Source Term}

Each molecular level is punped by a term of the form DETAIL (IV,J)*HFDOT, where HFDOT is the total rate of formation of HF molecules and DETAIL(IV,J) is the fraction of the total which is formed in the state with quantum numbers IV and $J$.

The values stored in the array. DEIAIL (IV,J) are given in Table 7.1 and Fig. 7.2. The values for $V=1,2$ and 3 are taken from chemiluminescence data of Pulanyi and koodall (1972b). Chemiluminescence data doesn't give the population for the vibrational ground state, $v=0$; so that had to be estimated. Following Cohen (1971) it was assumed that the total population with $V=0$ was one-half that with $V=1$. The remainder of the data suggested that the distribution shifted to higher $\mathrm{J}$ with lower $V$. Therefore the fraction of HF formed in the state $(0, J)$ was set equal to half that in the state $(1, \mathrm{~J}-1)$.

The value of HFDOT can either be determined by a chemical kinetics code such as MINOTAR or by a subroutine which calculates HFDOT, perhaps by means of an approximation. The second approach was taken in the IXION code, mainly for simplicity. A subroutine named CHEMIST calculated HFDOT and the relaxation constants, VDOT, RDOT and GAMMA2. (The FORTRAN listing of CHEMIST is given in Appendix F.) The 
algorithm for calculating HFDOT is:

$$
\begin{aligned}
\text { ERT } & =855 . / \text { TEMP } \\
\mathbf{R} 1 & =1.2 \mathrm{E} 14 * \operatorname{EXP}(- \text { ERT }) \\
\text { HFDOT } & =\mathrm{R} 1 * \mathrm{H} 2 * \mathrm{~F}
\end{aligned}
$$

where $R 1$ is the rate constant (Trotman-Dickenson, 1967) and $\mathrm{H} 2$ and $\mathrm{F}$ are the densities of $\mathrm{H}_{2}$ and $\mathrm{F}$ in moles/cc. The value, 855 .. is the activation energy divided by the gas constant. The value of $F$ varies because of consumption by the chemical reaction and because of production by the discharge. After examination of experimental current waveforms, the production of $F$ was approximated by $\sin (B t+\pi / 4)$ for values of $t$ up to $B t=3 \pi / 4$, and zero thereafter. A normalization constant, $B / 1.707$, set the value of the time integral to unity. The algorithm for $F$ atom density is

$$
B=1.25 \mathrm{E} 7
$$

$$
\begin{aligned}
& \text { ALPHA }=\mathrm{B} * \mathrm{TIME}+0.25 * \mathrm{PI} \\
& \mathrm{IF}(\mathrm{ALPHA} \cdot \mathrm{GT} \cdot \mathrm{PI}) \text { ALPHA }=0 \\
& \mathrm{FDOT}=\mathrm{FO} *(\mathrm{~B} / 1.707) * \mathrm{SIN}(\mathrm{ALPHA})-\text { HFDOT } \\
& \mathrm{F}=\mathrm{FOLD}+(\mathrm{TIME}-\mathrm{TOLD}) * \text { FDOT }
\end{aligned}
$$

with FOLD set equal to $F$ whenever TIME was updated. This algorithn assumes that the density of $\mathrm{H}_{2}$ remains constant. For most of the calculations, Fo was $1.25 \%$ of the density of $\mathrm{NF}_{3}$, or $6 \%$ of the density of $\mathrm{H}_{2}$. This amount of depletion is small compared to the uncertainty in the reaction rate. The value of $B$ is chosen to match the experimental width of the current pulse. 
To check the error introduced by assuming the density of $\mathrm{H}_{2}$ const:'nt, a constant temperature calculation was made with the MINOLAR code assuming $\mathrm{FO}=25 \%$ of the $\mathrm{H}_{2}$ density. The results are shown in Fi.g. 7.3. The solid line assumes constant $\mathrm{H}_{2}$ and the dotted line includes corrections for depletion of $\mathrm{H}_{2}$. The difference between the two is relatively small for the first 2 microseconds. In experiments the oscilloscopes were generally set for $2 \mathrm{microseconds}$ full scale. Therefore, depletion of $\mathrm{H}_{2}$ was not included in the IXION code.

Earlier chemical laser codes have treated the production of $H F$ and vibrational relaxation together. However, the two can be decoupled so long as the rate of formation of $\mathrm{HF}$ is not dependent on the population distribution of HF. The only known case where this is true is the postulated chain branching by the reaction

$$
\mathrm{HF}(\mathrm{v}>4)+\mathrm{F}_{2}+2 \mathrm{~F}+\mathrm{HF}(\mathrm{v}-4)
$$

This reaction will increase the concentration of $F$ atoms, thus accelerating the formation of HF. This has been studied in detail by Rapagnani (1972). The rate of this reaction has been estimated to be on the order of $10^{10}$ moles/cc-sec (Jensen, 1973). Rapagnani found that when using this rate the effect of Eq. 7.2 on the production of HF was negligible. Even at rates near bas kinetic $\left(10^{14}\right.$ mole/cc-sec) the rate of formation of HF was increased by less than an order of magnitude. Since experimental evidence shows the rate to be much smaller than $10^{14}$, reactions similar to Eq. 7.2 have 
been neglected in the IXION code. This permits formation of $\mathrm{HF}$ and vibrational deactivation of $\mathrm{HF}$ to be treated independently. Vibrational deactivation due to $\mathrm{Eq} .7 .2$ can still be treated, but not the additional quanticy of $F$ atoms.

7.3.2 Vibrational Relaxation Terms

The contribution of vibrational relaxation to Eq. 7.1 is given by

VDOT $*(V P * B N(J) * Y(M+3)-V * B N(J+1) * Y(M))$.

The first term represents the increase in population of state $Y(M)$ from the next higher state in the cascade, $Y(M+3)$. The factor $B N(J)$ gives the variation of the rate with rotational quantum number, and VP ( equals V+1) the variation with vibrational quantum number. The second term represents the loss in population of state $Y(M)$ due to vibrational relaxation. The factor VDOT depends on the density of collision partners and their relative efficiency in vibrational relaxation.

The form used for vibrational relaxation assumes that it is due to dipole interactions (Herzfeld and Litovitz, 1959). Vibrational relaxation then follows the same selection rules as radiation emission. The intensity dependence on vibrational and rotational quantum numbers is the same as for radiation and is discussed in more detall in the section on radiation.

The algorithm for calculating VDOT is

$$
\begin{aligned}
\text { VDOT }= & (7.1 \mathrm{E} * \mathrm{H} 2+1.6 \mathrm{E} 7 * \mathrm{~F} 3 \mathrm{~N}+2 . \mathrm{E} 6 * \mathrm{HE} \\
& +.72 \mathrm{E} 12 * \mathrm{~F}+1.42 \mathrm{E} 12 * \mathrm{HF})
\end{aligned}
$$


where the numerical values are the reaction rates at $300 \mathrm{~K}$ and the alphameric terms are the densities of the species which they represent. The rates are taken from Cohen (1971). The temperature variation was not included because it is more complex than that for other rates. The vibrational relaxation rate, at least for some species, appears to be minimum around $1000 \mathrm{~K}$ and higher for temperatures which are higher or lower than this. If the IXION code is to be run at temperatures other than 300K these rates should be changed. In the case of vibrational relaxation the equilibrium constant was neglected because the vibrational energy gap is large comparted to $\mathrm{kT}$. Even at $2000 \mathrm{~K}$ the upward rate is only a few percent of the downward rate, and for temperatures likely to be found in the laser the upward rate is negligible.

Cohen's rates were based on experiment (Bott and Cohen, 1971; Airey and Fried, 1971; Cohen, 1971; Chen and Moore, 1971; Hancock and Green, 1972; Hancock and Smith, 1971; Solomon et al., 1971) with consideration given to theory. Experiment indicates that the relaxation rate for $\mathrm{HF}$ is orders of magnitude faster than that predicted by the older theories (Herzfeld and Litovitz, 1959; Landau and Ieller, 1936; Rapp, 1960; Rapp and Kassal, 1969), which are satisfactory for many molecules. Newer theories which consider more complex interactions attempt to explain the anomously fast rotational relaxation of HF (Shin, 1970, 1971 and 1972; Thompson, 1972). 
The vibrational reiaxation rate for $\mathrm{H}_{2}$ used in calculating VDOT is for vibrational-translational transfer only. The vibrational states of $\mathrm{H}_{2}$ are near enough to resonance with HF that the transfer to $\mathrm{H}_{2}$ vibration is about twice the V-T rate. The algorithm required to calculate V-V transfer from $\mathrm{HF}$ to $\mathrm{H}_{2}$ is more complex than the $\mathrm{V}-\mathrm{T}$ transfer because vibrational energy stored as vibration in $\mathrm{H}_{2}$ can be returned to the $\mathrm{HF}$ at a later time. In some runs a worst case was calculated by assuming the $\mathrm{V}-\mathrm{V}$ rate but neglecting che return of the stored energy. Because the vibrational relaxation rate is slow, the higher rate had no visible effect for the chosen paraneters. Vibrational-rotational relaxation was neglected. as will be shown later, in most of the cases calculated the lasing was terminated by depletion of $F$ atom concentration before vibrational relaxation was significant. The situation would be dramatically different in the case of the chain reaction in an $\mathrm{H}_{2}+\mathrm{F}_{2}$ mixture.

\subsubsection{Rotational Relaxation Terms}

Rotational relaxation terms couple the state $Y(M)$ to the two adjacent states $Y(M+16)$ and $Y(M-16)$ having the same vibrational quantum number, but with rotational quantum numbers $J+1$ and $J-1$. (See Fig. 7.1) The basic rate is the rate to 80 from $\mathrm{J}$ to $\mathrm{J}-1$; the reverse rate, from $\mathrm{J}-1$ to $\mathrm{J}$ is related to this by an equilibrium constant. Thus there are four rotational relaxation terms, gain and loss 
terms to each of two adjacent states. Rotational relaxation was linited to adjacent states for computational simplicity. Multi-quantum jumps at decreased probability would be a more accurate physica] representation, but would greatly increase computation time.

The rotational relaxation terms are: RDOT $*(Y(M+16) * A J * D J(J+1)-(E Q R(I V, J) * D J(J+1)$ $+\mathrm{DJ}(J)) * Y(M)+E Q K(I V, J-1) * Y(M-16) * D J(J)$.

The factors, DJ, give the rotational quantum number dependence of the rate. The factor AJ is for computational purposes; it is set to 1.0 except at the highest $J$ values where it is set to 0.0 to cutoff extraneous values. The variables, EQK are the equilibrium constants.

The factor RDOT is the basic rate factor. The algorithm for RDOT is

$$
\begin{aligned}
\text { WIDTH } & =4.6 \text { E1 } 3 * \text { SQRT (TEMP) } \\
\text { RROT } & =0.5 * \text { WIDTH } \\
\text { RDOT } & =\text { RROT } *(H 2+\text { F3N }+ \text { FO }+ \text { HE })
\end{aligned}
$$

where the quantities in parenthesis are the densities of the collision partners. All collision partners are assumed equally: efficient at rotational relaxation because relative efficiencies are unknown. The coefficient, WIDTH, is obtained from experimental line-width data (Meredith, 1972). It repsesents a rate constant about 10 times gas kinetic collision frequency (assuming a gas kinetic cross section of $10^{-15} \mathrm{~cm}^{2}$ for HF). For most runs the value of WIDTH was loft constant, but RROT was treated as an adjustable parameter, 
because there was no experimental value available for RRoT. The best fit of calculated results with experiment was found with RROT $=0.5 *$ WIDTH, or 5 times gas kinetic. Physically RROT $\leq$ WIDTH because WIDTH represents those collisions where the molecule is knocked out of phase with the electric field, and RROT represents a collision which changes the rotational quantum number, and that clearly destroys the phase.

It need not be disturbing that the cross section is greater than so-called gas kinetic. Gas kinetic cross sections are usually determined from collisions which alter the momentum of the molecule, Here we are dealing with torques resulting from long range dipole forces, and it is reasonable that they should have a larger cross section.

The ratio between the upward and downward rates is derived by considering the equilibrium distribution. At equilibrium the population distribution remains unchanged with

$$
\mathrm{N}_{\mathrm{J}}=\mathrm{N}_{\mathrm{J}-1} \cdot\left(\frac{2 \mathrm{~J}+1}{2 \mathrm{~J}-\mathrm{T}}\right) \mathrm{e}^{-\Delta E / k T}
$$

where $\mathrm{N}_{j}$ is the number of molecules in state $\mathrm{J}, 2 \mathrm{~J}+1$ is the degeneracy of state $\mathrm{J}$, and $\Delta \mathrm{E}$ is the energy difference between states $J$ and $J-1$. To maintain equilibrium we must have

$$
\mathrm{k}_{+} \mathrm{N}_{\mathbf{J}-1}=\mathrm{k}_{-} \mathrm{N}_{\mathbf{J}}
$$

where $k_{\text {- }}$ is the downward rate and $k_{+}$the upward rate. Substituting we get

$$
k_{+}=k_{-} \cdot\left(\frac{2 J+I}{2 J-I}\right) e^{-\Delta E / k T}
$$


The factor on the right hand side is the equilibrium constant. Ạthough Eq. 7.4 is derived at equilibrium it is valid under most conditions even when the populations are not in equilibrium .

Within the IXION code the equilibrium constant is calculated by

$$
\begin{aligned}
& \operatorname{ARG}=\left(\operatorname{TER}{ }_{1}(I V, J)-\operatorname{TERM}(I V, J+1)\right) /(R * T E M P) \\
& \operatorname{EQK}(I V, J)=\operatorname{EXP}(\operatorname{ARG}) / G R(J+1)
\end{aligned}
$$

Where $\operatorname{TERM}(I V, J)$ is the energy $\left(\mathrm{cm}^{-1}\right)$ of the vibrationalrotational state with quantum numbers IV and $J$ (see Table AZ, Appendix A), and $R$ is the gas constant, $(0.695 / \mathrm{cm}-\mathrm{K})$. The factor, $G R(J+1)$, is the degeneracy ratio, $(2 J-1) /(2 J+1)$.

The dependence of the rotational relaxation rate on rotational quantum number is given by the array, DJ(J). Establishing the proper values for DJ was one of the more difficult tasks in setting up the model. No experimental data on the $J$ dependence of the rotational relaxation rate in HF could be found. There was also no directly applicable theory. Polanyi and Woodall (1972a) had fit a simple model. to experimental $\mathrm{HCl}$ rotational relaxation data. An attempt was made to estimate the rate with a classical model assuming that the dipole-dipole interaction was dominant. Incomplete results verified that the rotational relaxation rate decreased at higher $\mathrm{J}$ as did the $\mathrm{HCl}$ experimental data. In the process of obtaining line-broadening coefficients it was noted that experimental line widths for HF (Meredith, 1972) varied with' $\mathrm{J}$ in the same manner as Polanyi and Woodall's best 
mode1. This is not surprising, because line broadening is caused by two kinds of collisions: those resulting in a change of quantum number and those which merely change the phase of the oscillation. Clearly the line broadening collision rate is an upper limit on the rotational relaxation rate. For transitions in the visible region of the spectrum phase changing collisions dominate because the transition energy is much larger than $\mathrm{kT}$. In the microwave spectrum line broadening is almost encirely due to changes in quantum number because the transition energy is much less than $\mathrm{kT}$. The transition energies for HF are comparable to $\mathrm{kT}$ and both types of collision are important.

The choice of the rotational relaxation rate is discussed in more detail in Appendix $D$. The model obtains the $J$ dependence of the rotational relaxation rate from experimental line-broadening data for (Meredith, 1972). The magnitude of the rotational relaxation rate, RROT, was taken as some fraction of the collision rate for line broadening. The fraction was taken as an adjustable fraction, with $1 / 2$ being a likely fraction. The variation of the rate, $D J(J)$ with $\mathrm{J}$ is given in Fig, 7.4 and Table 7.2.

7.3.4 The Radiation Coupling Terms The first line of Eq. 7.1, $\mathrm{YDOT}(\mathrm{M})=2 . * \mathrm{CPH} *(\mathrm{AV} 6 * \mathrm{Y}(\mathrm{M}+1) * \mathrm{Y}(\mathrm{M}+2)-\mathrm{AV} 1 * \mathrm{Y}(\mathrm{M}-2) * \mathrm{Y}(\mathrm{M}-1))$, consists of two terms of the form $2 \mathrm{pq} \cos \phi$ of Eq. 6.40c. The first term represents the increase in population due to laser radiation from the next higher state in the cascade. 
The second term represents loss of population to the next lower state via laser radiation. The coefficients AVI and AV6 are set equal to 1.0, except at the top and bottom of the cascade where they are used to cut of extraneous values. The variables $\mathrm{Y}(\mathrm{M}+1)$ and $\mathrm{Y}(\mathrm{M}-2)$ represent polarizations while $Y(M+2)$ and $Y(M-1)$ represent electric fields.

The factor, $\mathrm{CPH}$, represents the steady state value of $\cos \phi$, where the steady state value is given by $\mathrm{Eq} \cdot 6.33 \mathrm{a}$. The algorithm used to calculate $\mathrm{CPH}$ is

$$
\begin{aligned}
\text { TFH } & =(4.7124 E 10 / \text { CAVLN }) /(\text { CAV1 }+ \text { GAMMA2 }) \\
\text { CSQ } & =1 . /(1 .+ \text { TPH } * 22) \\
\text { CPH } & =\operatorname{SQRT}(\mathrm{CSQ}) .
\end{aligned}
$$

The first line corresponds to Eq. $6.33 a$ with w-w set to half the intermode spacing (maximum detuning). The factor, CAV1, corresponds to $\beta$ in Eq. 6.33a, GAMMA2 corresponds to $Y^{\prime}$, and IPH to $\tan \phi$. CAVLN is the cavity lerigth. The remainder of the algorithm is a trigonometric identity.

As written, $\cos \phi$ is actually double valued since $\tan \phi$ is positive in the first and third quadrants. In the first quadrant cos is positive and represents the case with positive inversion. When the inversion is negative $\phi$ is in the third quadrant so that $\cos \phi$ is negative and the inversion tends to restore itself by absorbing light from the fields. The variables $p$ and $q$ are lengths of a polar vector and may not be negative. hithin the code the equivalent results are calculated in a different manner: $\cos \phi$ remains positive and the terms representing the 
magnitude of the fields change sign. The equivalent of positive $\cos \phi$ is for the signs of the electric field and polarization to be the same. When the signs differ it is equivalent to $\cos \phi$ being negative.

Before using the steady state value in the code, tests were made using the complete time variation of $\phi$. It turned out that spent most of its time near one of the steady statevalues and jumped rapidly to the other at appropriate times. The full variation of tended to smooth out the spiking somewhat and had little other effect on the waveforms, but it did require an order of magnitude more computer time. As a result the steady state value was used in the code.

\subsection{The Polarization Equation}

The differential equation for the polarization is

$$
\stackrel{\circ}{\mathrm{p}}=\mathrm{r}^{\prime} \mathrm{p}+\mathrm{b}^{2} \mathrm{q}\left(\mathrm{N}_{2}-\mathrm{N}_{1}\right) \cos \phi
$$

The equivalent equation in the IXION code is $\mathrm{YDOT}(M+1)=-\operatorname{DOMEGA} * \mathrm{Y}(\mathrm{M}+1)+\mathrm{VP} * \mathrm{BN}(\mathrm{J}) * \mathrm{BSQ} * \mathrm{~N}^{\prime} \mathrm{AVNUM} * \mathrm{CPH}$

$$
*(Y(M+2) *(Y(M+3)-G R(J) * Y(M))+Y(M+3)) \cdot(7.3)
$$

This form assumes that there is only one laser mode for each possible laser transition. This will be discussed in detail below.

\subsubsection{Damping Term}

The lirst term, DOMEGA*Y(M+1), is the polarization damping term. The variable $\mathrm{Y}(\mathrm{M}+1)$ represents the polarization and the coefficient DOMEGs is the linewidth For a Voigt profile combining the Doppler and collisional linewidths. The algorithm for DOMEGA is 


$$
\begin{aligned}
& \text { DPK }=2400 . * \operatorname{SQRT}(\text { TEMP }) \\
& \text { WAVNUM }=\operatorname{TERM}(I V+1, J-1)-\operatorname{TERM}(I V, J) \\
& \text { KIDTH }=4.6 E 13 * \text { SQRT }(\text { TEMP }) \\
& \text { GAMMA2 }=\text { WLDTH } *(\mathrm{H} 2+\mathrm{F} 3 \mathrm{~N}+\mathrm{FO}+\mathrm{HE}) \\
& \text { DSQ }=(\text { GAMMA } 2 * \text { DJ }(J+1)) * 22+(\text { DPK } * W A V N U M) * 2 \\
& \text { DOMEGA }=\text { SQRT(DSQ) }
\end{aligned}
$$

The first term in DSQ is the square of the collisional linewidth and the second is the square of the Doppler linewidth. The coefficient DPK is the Doppler coefficient and hAVNUM is the reciprocal of the wavelength, with TERM(IV,J) being the energy in $\mathrm{cm}^{-1}$ of the state with quantum numbers IV and 3 . The linewidth coefficient, WIDTH, is set to the experimental value for HF self-broadening (Merelith, 1972). The collisional linewidth, GAMMA2, is obtained by multiplying WIDTH by the densities of the collision partners. Due to lack of other data, all collision partners are assumed to have the same efficiency. The variation of collisional broadening with rotational state is contajned in the factor $D J(J+1)$. This factor was described in detail in Section 7.3.3.

The natural linewidth was neglected because il is several orders of magnitude smaller than ej ther the collisionnl or Doppler linewidths. The collisional and Doppler linewidths are added in quadrature for reasons to be described below. The laser cavity hals many modes, separated by $C / 2 \mathrm{~L}$, noar the frequency of the molecular resonance. A colli. si onally broadened 1 ine and a boppler broadened line interact lillerently with these modes. If the line is collisionally 


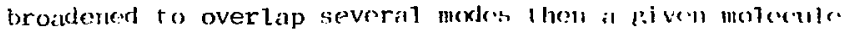
underpoes soveral phase changing, collisions duriul, a roud-

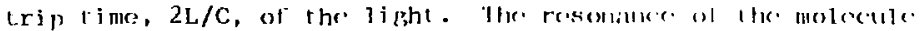
i.s therefore coupled to several mekles, and oflen lasine: will occur only on the mode with the highest gain, or on a fow modes. On the other hand, if a line is Doppler broadened to overlap several modes the molerules can be divided into velocity groups each of which interact with one mode, but not with each other. In this case all of the modes with gains greater than unity can lase. When collisional and Doppler Tinewidths are comparable the collisions couple ad jacent velocity groups so that the modes are coupled. A rigorous treatment of linewidths would involve several mades for each laser transition and would greally increase the complexity of the calculation. For computational simplicity it was decided to consider only a single mode on each transition. Also, the calculations were being compared with experiments using an $8 \%$ reflectivity mirror which makes the modes rather wide and allows coupling betweren morles, complirating the interaction bur. making mode structure loss important.

If we assume that only il siugle ravity noxle is lasing for each transition we only need lo calculate the gain at line-center, which is inversely proportional to the linewidth. The lineshape is a Voigt profile, which is basically a convolution of the Gaussian profile of the Joppler broadener line with the Lorenty profile of thr collision broadened line. Because variances add under runvolution the total 
Iinewidth can be estimated from the sum of the squares of the two linewidths. Total linewidths were determined in this way for the IXION code. The resulting linewidth was then used to calculate an effective damping coefficient, DOMEGA, for the polarization.

\subsubsection{Coupling to the Population Inversion}

The string of factors $V P * B N * B S Q * k A V N U M$ in $\mathrm{Eq} .7 .3$ is equivalent to $\mathrm{b}^{2}$ in $E q, 6.40 \mathrm{~b}$. The variable $Y(M+2)$ is equivalent to $q$, and $Y(M+3)-G R(J) Y(M)$ is equivalent to $N_{2}-N_{1}$, where $G R(J)$ is the degeneracy ratio, $(2 J-1 / 2 J+1)$. The last term requires some comment.

Equations $6.40 \mathrm{a}$ and $6.40 \mathrm{~b}$ are written in a form which neglects the noise introduced by spontaneous emission. The equations have two steady state solutions with one of them being $p=q=0$. If the equations are started at these values they will remain there. This is not a physically realistic situation, and to match physical reality we must add a term to represent the noise generated by spontaneous emission. The spontaneous noise term was lost in the derivation of Eq. 6.40 at the point where we made the semi-classical approximation on expectation values, $\left\langle\sigma_{1}{ }^{-} a_{1}{ }^{+}\right\rangle=\left\langle\sigma_{1}{ }^{-}\right\rangle\left\langle a_{1}{ }^{+}\right\rangle$. We now reintroduce it phenomenologically. In the usual treatment with the radiation field treated as a harmonic oscillator with quantum number $\mathrm{n}$ is proportional to the square of the electric field, i.e. $q^{2}$, but we have $q$ in $\mathrm{Eq} \cdot 6.40 \mathrm{~b}$. There is no simple way to get $\mathrm{q}^{2}+1$ so we use $\mathrm{q}+1$ and rewrite $\mathrm{Eq} \cdot 6.40 \mathrm{~b}$ as 


$$
\stackrel{\circ}{\mathrm{p}}=-Y^{\prime} p+(q+1) \mathrm{N}_{2}-\mathrm{qN}_{1} .
$$

Physically this represents a spontaneous polarization of a molecule which results in an electric field for ore photon.

If this treatment doesn't give exact quantitative results for the noise it will have little effect on the model. The noise is some 20 orders of magnitude below the steady state lasing level. Once threshold inversion is reached, the power increases exponentially with time until it overshoots the steady state value. The only effect of a change in noise level is a change in the time to reach steady state, since steady state power depends on the pump rate, not the initial conditions. This is a result of the non-linear nature of Eqs. 6.40. An order of magnitude change in the noise level will simply change the time to reach steady state by about $5 \%$. Therefore, we need not be concerned about small errors, if any, in the treatment of the noise term.

The factor $G R(J+1)$ accounts for the fact that there are $2 J+1$ values of the anagnetic quantum number, $m$. The selection rule is $\Delta \mathrm{m}=0$ so that we have an inversion when $\mathrm{N}_{2} /(2 J-1)-\mathrm{N}_{1} /(2 J+1)$ is positive. In the absence of a strong magnetic field there are 2J-1 transitions at a single frequency so the appropriate form for the inversion is $\mathrm{N}_{2}-\mathrm{GR}^{\mathrm{H}} \mathrm{N}_{1}$ where $\mathrm{GR}$ is $(2 J-1) /(2 J+1)$. Any additional rotational dependence of the gain is included in the factor BN(J).

The values of $\mathrm{BN}(J)$ are taken from calculated matrix elements of Meredith (1972). The calculations are for a Morse oscillator and have a different rotational distribution 
than a harmonic oscillator. The vibrational dependence is the same: name1y, proportional to the vibration quantum number, $v$, of the upper state. In the code the vibrational dependence is treated by the factor VP. The values of $B N(J)$ and $G R(J)$ are shown in Fig. 7.5.

The product of factors VP*BN $(J) * B S Q *$ WAVNUM is equivalent to the $\mathrm{b}^{2}$ of $\mathrm{Eq} \cdot 6.40 \mathrm{~b}$. The dimensions of the variables in Eq. $6.40 \mathrm{~b}$ are chosen so that

$$
b^{2}=\left(\mu^{2} / 6 h^{2} \varepsilon\right) \cdot \not h w
$$

where $\mu^{2}$ is the dipole moment and $\varepsilon$ the permittivity of the medium in S.I. units. The variable WAVNUM equals $1 / \lambda$ so is proportional to hw. The coefficients VP and BN were normalized to equal 1.0 for the $\mathrm{V} 2-1, \mathrm{P}(6)$ transition, so that

$$
\mathrm{BSQ}=\left(\mu^{2} / 6 \mathrm{~h}^{2} \varepsilon\right)
$$

for the v2-1 $P(6)$ transition with WAVNUM in units of $\mathrm{cm}^{-1}$. The numerical value of BSQ obtained from Meredith (1972) is $4.7 \times 10^{-6}$. This corresponds to a dipole moment of 0.125 Debye.

The factor $\mathrm{CPH}$ corresponds to $\cos \phi$ in Eq. $6.40 \mathrm{~b}$. This was discussed in detail in Section 7.3.4.

$$
\begin{aligned}
& 7.5 \frac{\text { The Electric Field Equation }}{\text { The electric field equation is }} \\
& \dot{q}=-B q+p .
\end{aligned}
$$

The FORTRAN version in the IXION code is

$$
\operatorname{YDOT}(M+2)=-\operatorname{CAV} 1 * Y(M+2)+\text { FILL } * \underline{Y}(M+1)
$$

where $\mathrm{Y}(\mathrm{M}+1)$ is the polarization and $\mathrm{Y}(\mathrm{M}+2)$ the electric field. 
The coefficient FILL occurs in Eq. 7.5 because the active medium doesn't fill the entire volume of the cavity, as was assumed in Eq. 6.40a. In the present case FILT is just the gain length divided by the cavity length. There is some uncertainty in determining the gain length because of the non-uniform spatial distribution of the discharge. Experimental observations of the beam shape indicated that the discharge region consisted of 50 cylindrical discharges of about $2.5 \mathrm{~mm}$ diameter at half intensity. Thus the gair length was estimated at $13.5 \mathrm{~cm}$. Further uncertainty arises because the low reflectivity output mirror causes the intensity to vary along the axis, causing a spatial variation in the threshold inversion. With an $8 \%$ output mirror the inversion will vary a factor of 2. Fortunately these uncertainties are not critical. The total power output depends linearly on the estimated active volume multiplied by the initial estimated $F$ atom density. Time delays in the waveform have only a logarithmic dependence on the value of FILL.

Clearly the coefficient CAVl is equivalent to $\theta$ which equals $1 / 2 \tau_{c}$, where $\tau_{c}$ is the lifetime of a photon in the cavity. Photons are lost from the cavity by transmission through the output mirrors, absorption loss at mirrors and windows, scattering losses in the gas and diffraction due to finite apertures in the system. Scattering and diffraction losses are a few percent or less in most experimental setups. Window losses, especially from reflection out of the cavity, 
could be as large as 10 or 20 percent for unfavorable polarizations. However, we are comparing calculations with experiments which used low reflectivity mirrors, makins other losses insignificant. If we consider only mirror losses we get

$$
B=\frac{1}{2 \tau_{c}}=-\frac{c \ln \left(P_{1} P_{2}\right)}{4 \bar{L}_{c}}
$$

where $p_{1}$ and $\rho_{2}$ are mirror reflectivities, $L_{c}$ is the cavity length, and $c$ is the velocity of light. To the extent that other losses can be Iumped, rather than spatially distributed, they car be lumped into one of the mirror reflectivities. The output intensity will be given by

$$
I_{\text {out }}=\left(1-p_{2}\right) I_{\text {cavity }}
$$

and the other losses can be Iumped under $\rho_{1}$.

$$
\begin{aligned}
& \text { The algorithm for calculating CAV1 and FILL is } \\
& C=3 . E 10 \\
& \text { RHOl }=0.08 \\
& \text { RHO2 }=1.0 \\
& \text { CAVLN }=65 . \\
& \text { GAINLN }=13.5 \\
& \text { CAV1 }=-.25 * \text { C*ALOG (RHO1 *RHO2)/CAVLN } \\
& \text { FILL }=\text { GAINLN/CAVLN }
\end{aligned}
$$

The variable names correspond to those in Eq. 7.6. Reflectivity RHO1 was set equal to unity and RHO2 to 0.08 . 
Because of the low value of RHO2 the output power is $92 \%$ of the power in the cavity at the output mirror. As a result the output from the code gave the power in the cavity rather than the actual output power. This should be corrected if different output mirrors are to be compared.

The code calculates power output per $\mathrm{cm}^{2}$ of beam area. Examination of the experimental bean intensity pattern indicated that it was oval, about $2.5 \mathrm{~mm}$ wide and $15 \mathrm{~mm}$ hi.gh at half intensity. The effective area of the experimental beam was estimated to be $0.4 \mathrm{~cm}^{2}$. A calculated beam output of $2.5 \mathrm{~mJ} / \mathrm{cm}^{2}$ thus corresponds to an esjerimental output of $1 \mathrm{~mJ}$, a typical value.

\subsection{Simulation of Detector Response}

The experimental waveforms were obtained using a detector with a rise time of about $30 \mathrm{~ns}$; this was simulated in some computer runs to give a more accurate comparison.

The simplest form for detector response to a unit impulse function is $D(t)=e^{-t / \tau}$ where $T$ is the detector rise time and the function is normalized by the factor $1 / \tau$. If the detector response function is $D(t)$ and the Iaw waveform is $f(t)$, the observed waveform $g(t)$ is obtained by convolution of $D(t)$ with $f(t)$, i.e.,

$$
g(t)=\int_{0}^{t} D(t-u) f(u) d u
$$

where $u$ is a dummy variable with dimensions of time. 
This is rasi $1 \mathrm{y}$ implementerd numerically i t the raw wavrluom i bivrn ats a scaluence of values at discrete times. the tint: step need not be uniform, but it is preferable that it be loss than $t$. The lact or $D(t-u)$ can be written as a product

$D(t-u)=1 / \tau e^{\left(t-u_{n}\right) / \tau} e^{\left(u u^{-u} n-1\right) / \tau} \ldots e^{\left(u_{2}-u_{\tau}\right) / \tau} e^{\left(u_{1}-u\right) / \tau}$.

For the most recent value of $f(t)$ the integral cau he approximated by

$$
\left(1-r^{-\Delta t / \tau}\right) \&\left(t_{0}\right) \text {. }
$$

(This form is chosel rather than $\Delta t L(t) / \tau$ because it gives a more: atculrate result when $f(t)$ remains constant.) On the next time step the same element has a contribution

$$
\therefore^{-\Delta t_{1} / \tau}\left(1-e^{-\Delta t / \tau}\right) f\left(t_{0}\right),
$$

so that $\mathcal{D}\left(1-t_{0}\right)$ has become a product. With each time step nother rerm is included in the product, yet the runction $i\left(t-t_{0}\right)$ remains exact and the only approximation is in the initial step. Any cror introduced in the initial step is danjped out breause $u\left(t-t_{0}\right)$ decreases momoronically.

The aleorithm is simplor than the derivation, and wats initially obtained huristically. The algorithm, rrpeillod sich time slep, isi

$$
\begin{aligned}
& n t=1 . / 30 . E-9 \\
& \Lambda 1 t=\text { IET } *(\text { TIME:-TOI, }) \\
& \text { JOl,i) = TINE: } \\
& \operatorname{SMIIIT}=\operatorname{EXP}(-\mathrm{AH})
\end{aligned}
$$


bo 230 L.S $=1,320$

230 SMOOTH(L.S $)=Y(L S)+$ SNI! $1 \%(\operatorname{SHMOTH}(L . S)-Y(1 S))$

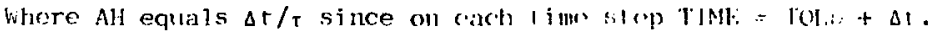

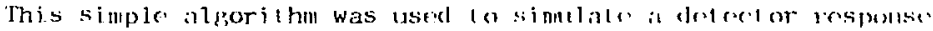
of 30 nimoseronds.

\subsection{Tomperature Rise}

The Lomperalure has bern hold constant in most rums with the IXION code, largely out of ignorance of the appropriate temperature dependence. These are two sources of temperalture variation, the heat of reaction and the imeating by the electrical discharge.

Heat ing due $t$ o the exol hermic reaction is easy to estimate. The reaction $\mathrm{F}+\mathrm{H}_{2}+\mathrm{HF}+\mathrm{H}$ is exothermic by $32 \mathrm{kcal} / \mathrm{mole}$. For the exp.. ments where waverorms were compared to calculations it appears that the partial pressure of HF was about $1 \%$ of the total pressure. Thus the heat of reaction yicilds ab at $320 \mathrm{cal} / m o l e$ of the total inixture. About half of this is radiated as laser energy. The specilic heat of the: mixture is about $13 \mathrm{Gibbs} / \mathrm{mole}$ so the remperature rise should be about $12 \mathrm{~K}$, a rather small risc.

On the other hand, experiment (Pearson, et a1., 1973a) shows that the 1rser output was about $1 \%$ of the electrical discharge energy. If the majorily of the discharge energy, about $30 \mathrm{kcal} / \mathrm{mole}$, went into heating the gases the temperacure rise would be at least $1200 \%$. Analysis of experimental Lime constants shows that the rate-rorlstirl:s are appropriate 
to a tomperature change or less than $200 k$ (rearson, et al., 1972b). The majority of this discharge energy is not accounted 5or.

The discharge energy can be partitioned into a number of modes: ionization, dissociation, radiation, rlectronic, vibrationa 1 and rotational excitation and translational energy. The chemical reaction rate depends inainly on the translational temperature because the reactants need translational energy to overcome the potential barrier. A simple calculation shows that little of the discharge energy gocs initially into translatjonal energy.

In thr usual modrl of an electric discharge the r. lectron frilns a low tens of eilectron volt: irom the electric I ield hethoen collisions. When it collides with an atom or molecule it gives up this cnergy. Ir also gives up some momentum, and, since momentum must be consorved, the niolecinle pains momentum. The momentum of the electron before collision has the magnitude

$$
F_{\epsilon}=\sqrt{2 m_{e} E_{e}} .
$$

where $F_{c}$ is the hinetic enerpy of the electron, $m_{r}$ its mass

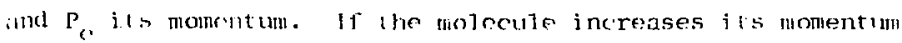
the trans lational ruerpy rhange is

$$
\Delta H_{1}=\left(B_{M}^{\prime} \cdot P \cdot P_{M}{ }^{2}\right) 2 M=\Delta M_{M}\left(I_{M}^{\prime}+I_{M}\right) / 2 M
$$

where $B_{M}^{\prime}$ is the momentum of the moteculd alter the rollision, l'p the momentum boloro collision, and $M$ the moleculas mess. $11 \quad \Delta P_{N 1}=P_{C}<P_{N}$ 


$$
\Delta \mathrm{L}_{2}=\sqrt{\mathrm{E}_{2} \mathrm{E}_{1} \mathrm{~m} / \mathrm{M}} \text {. }
$$

Typically $E_{0}-1500 \mathrm{kT}$ and $\mathrm{L}_{1}=3 / 2 \mathrm{k}$, If the c1ectron

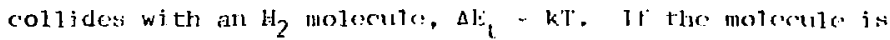
$\mathrm{Nl}_{3}$ the enerby change: is aboul $1 / 6 \mathrm{kt}$. in either calse, Lum increase in molecular kinetic enerpy i.s loss than $10^{-3}$ of the electron energy. The remainder of the electron's kinetic energy inust go into electronic or vibrational excitation of the molecule. Conservation of angular monentum requires the change in rotational energy to be comparably small.

Eventually, the electronic and vibrational eneigy must be transferred to translational energy, be radiated away, or be dissipated hydrodynamically. Typically vibrationalco-translatjonal energy transfer requires thousands of collisions, but the laser pulse is complete by the time a few hundred collisions have occurred, so the translational tomperature remains small. It j.s probably fortuitous that the increase in kinetic energy of $\mathrm{H}_{2}$ gives an erfectjve temperature of about $500 \mathrm{~K}$, the remperature which estimates the pulse width correctly (Pearson, et al., 1972b).

Calculating the correct translational temperature is quite difficult, so calculations with the LXION code assumed a constant temperature, $300 \mathrm{~K}$ in most cases.

\subsection{Codes to Rearrange Output}

The output of the IXION corle is a disc file, SCRATCH. The file is arranged in blocks, with each block containing the values of all powers and populations at one time, along 
with a few auxiliary variables such as temperature and the step number. The interval for printouts is chosen so that the file contains between 100 and 1000 such blocks. An auxiliary file, GUIDE, gives statistics on SCRATCH so that editing coes can read it easily.

An obvious way of displaying data from a computer code is to have time histories of the variables. Two codes were written to produce graphical and tabular time histories from the data contained in the disc file, SCRATCH. One of these codes, COLLAGE, plotted the waveforms which will be shown in Fig. 8.1. A second code, EDITB, gave = more complete output. This output was arranged by cascades and showed the inversions and total populations for each inversion. If any of the transitions in the cascade lased, the lasing waveforms are also plotted. In addition, twenty snapshcts of the population distributions are plotted. Because of the length of the SCRATCH file, (500K decimal words), the EDITB code had to do extensive sorting to efficiently transform from data blocked in time steps to time sequences for each of several hundred variables. In addition to the graphs EDITB provided tables of the time history of each variable.

The snapshots of population distributions suggested that a movie might be a useful way to display the data in order to increase our understanding of the physical processes. A code was written to make such a movie (Creighton, 1974). Single frames from the movie will be used in Section 8 to illustrate the operation of the laser. 
TABLE 7.1. Fraction of HF going into aach vibrational-rotational state as

a result of the reaction

$$
\mathrm{F}+\mathrm{H}_{2}+\mathrm{HF}+\mathrm{H}
$$

\begin{tabular}{lllll}
$J$ & $v=0$ & $v=1$ & $v=2$ & $v=3$ \\
0 & .0 & .0 & .0 & .01158 \\
1 & .0 & .0 & .0 & .05580 \\
2 & .0 & .0 & .00673 & .06784 \\
3 & .0 & .0 & .02537 & .05742 \\
4 & .0 & .00161 & .04816 & .03357 \\
5 & .00080 & .00771 & .06576 & .01436 \\
6 & .00385 & .01766 & .07819 & .00232 \\
7 & .00883 & .03082 & .07975 & .0 \\
$B$ & .01541 & .03162 & .07094 & .0 \\
9 & .01581 & .02697 & .05282 & .0 \\
10 & .01348 & .01846 & .03780 & .0 \\
11 & .00923 & .01461 & .02537 & .0 \\
12 & .00730 & .00851 & .01605 & .0 \\
13 & .00425 & .00225 & .00725 & .0 \\
14 & .00112 & .0 & .00259 & .0 \\
15 & .0 & .0 & .0 & .0 \\
\hline & & & & \\
\hline
\end{tabular}


TABLE 7.2. Values of the vector, $\mathrm{DJ}(\mathrm{J})$, which give variation of collieionel lineridth and rotational relaxation rate with rotational quantun nuber, $J$.

\begin{tabular}{llrlll}
$J$ & $D J(J)$ & $J$ & $D J(J)$ & $J$ & $D J(J)$ \\
\hline 0 & 0.0 & 7 & 0.1169 & 14 & 0.009673 \\
1 & 0.525 & 8 & 0.08187 & 15 & 0.006776 \\
2 & 0.515 & 9 & 0.05735 & 16 & $0.0047 / 5$ \\
3 & 0.430 & 10 & 0.04017 & 17 & 0.003325 \\
4 & 0.340 & 11 & 0.02814 & 18 & 0.002329 \\
5 & 0.2382 & 12 & 0.01971 & 19 & 0.0011632 \\
6 & 0.1668 & 13 & 0.01381 & 20 & - \\
\hline
\end{tabular}




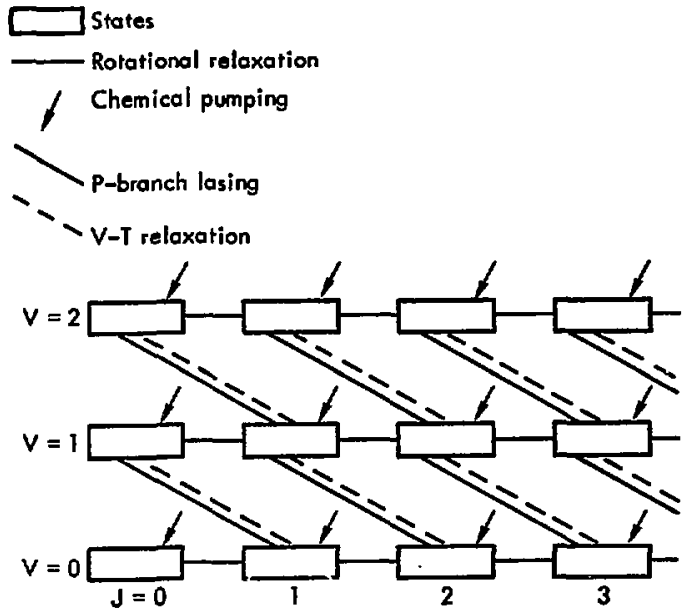

Fig. 7.1 Schematic diagram of the IXION model showing the individual states and the rates which couple them. 


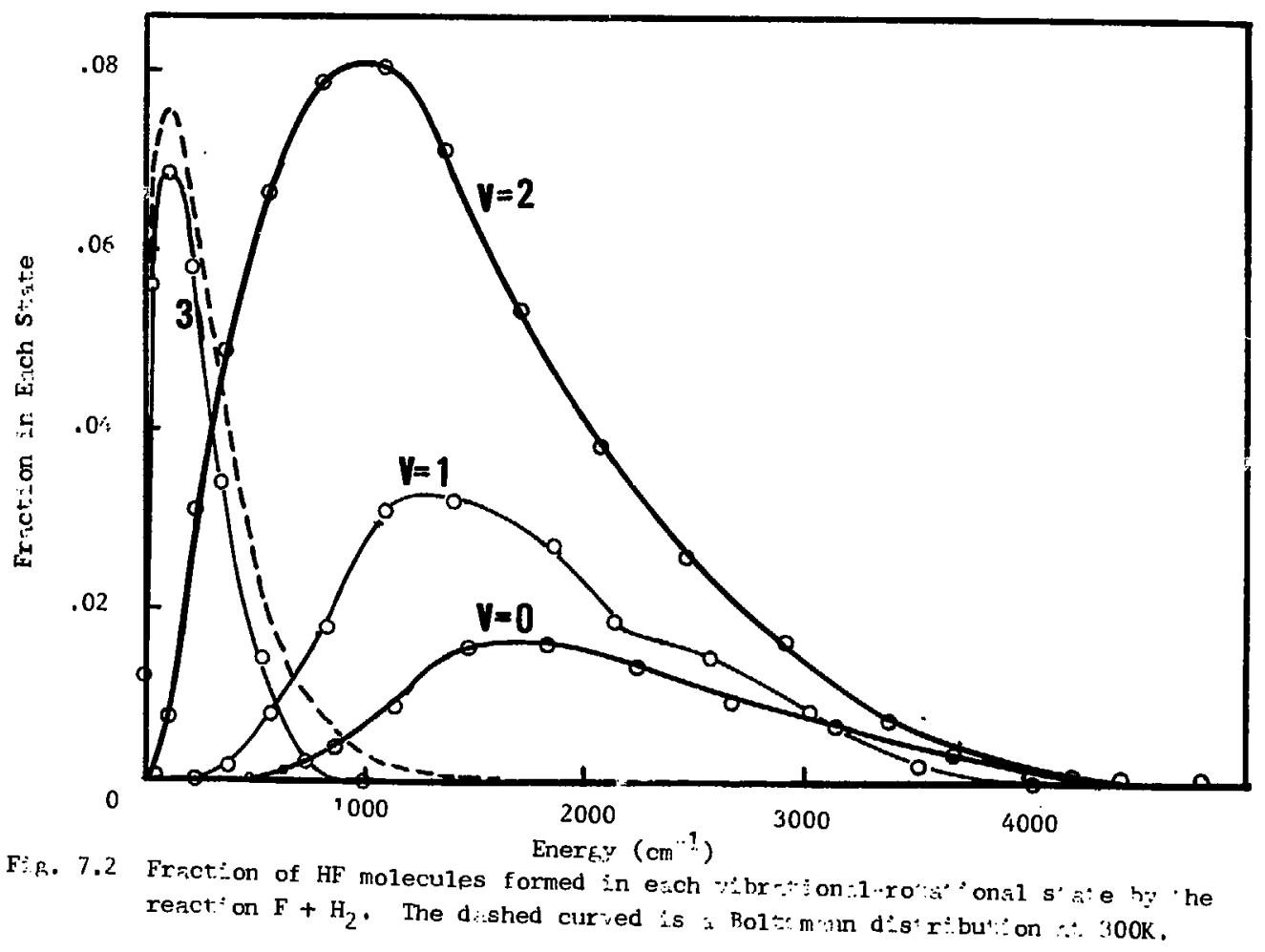




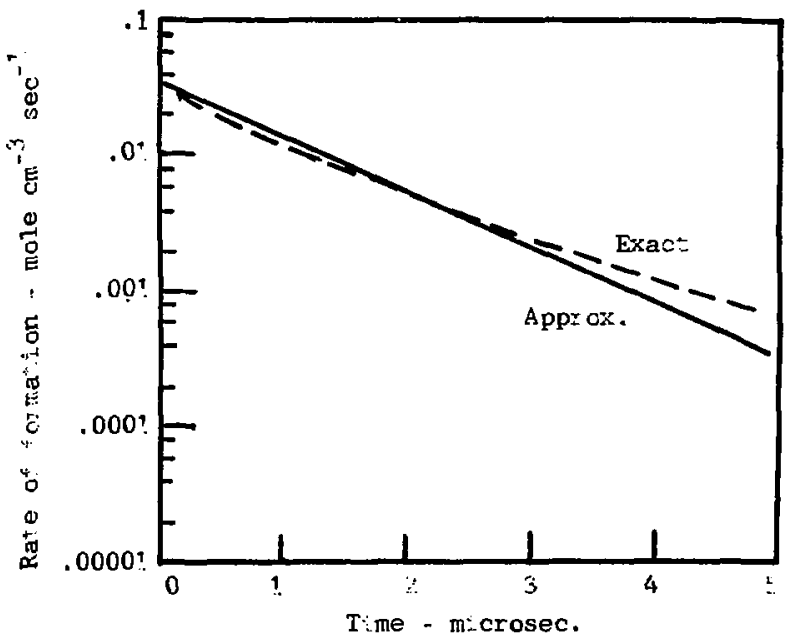

Fig. 7.3 R:e of sormation of $\mathrm{HF}$ in the pulsed $\mathrm{NF}_{3}+\mathrm{H}_{2}$ 1sser. The solid line represent.s the spproximation of nerilesting consumption of $\mathrm{H}_{2}$. The dotted line is an exac: nume: ical resul: where about $2: \%$ o: the $\mathrm{H}_{2}$ is consumed. 


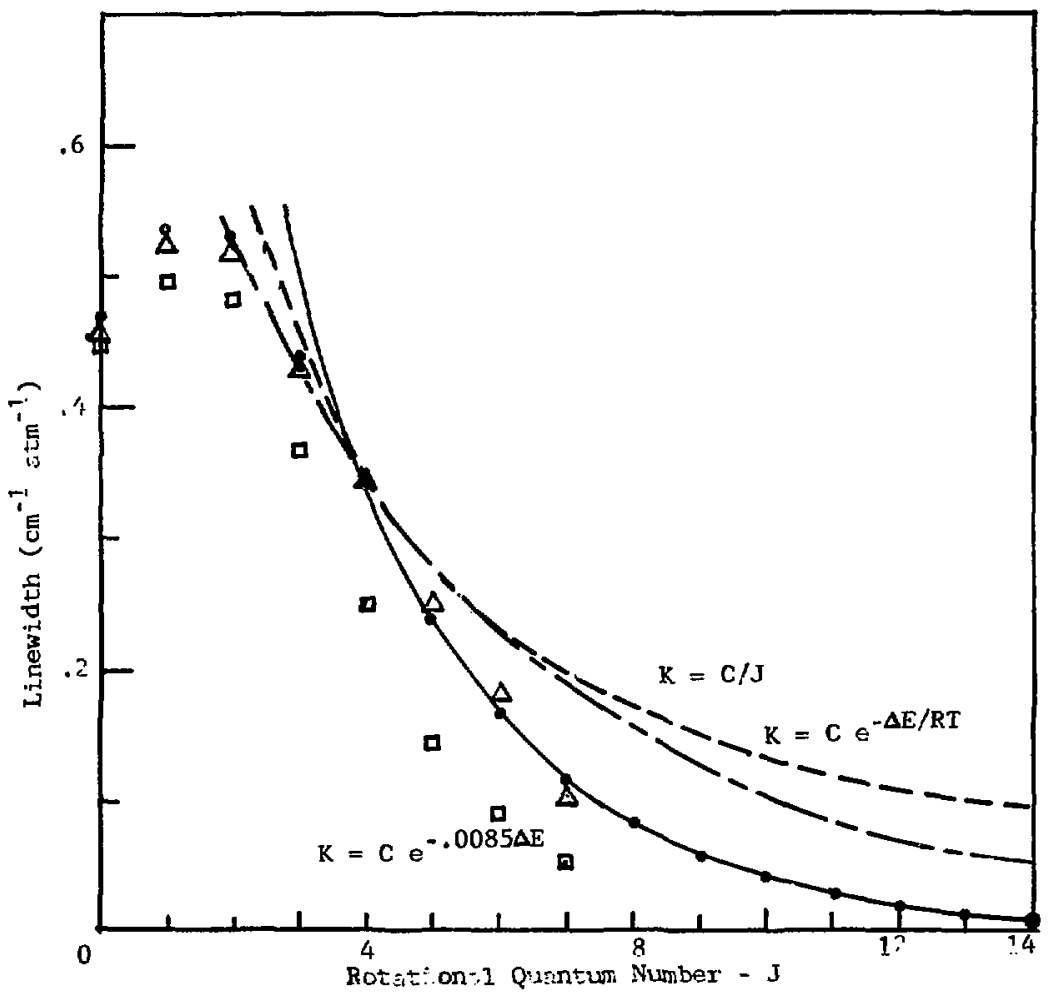
$\Delta$ Messured.
- Anderson theory
from Meredi.n (197\%)

- Values used in IXION code

Fig. 7,4 jependence of HF self-broadened linewidth on rotational quantum number: Se:eral curve $=i m s$ are shown normalized to the 1inewidth $3: J=4$. At 15 Torr and $300 \mathrm{~K}$ the appler wid:h is approximately etual to the collisional linew:dth at $J=7$. 


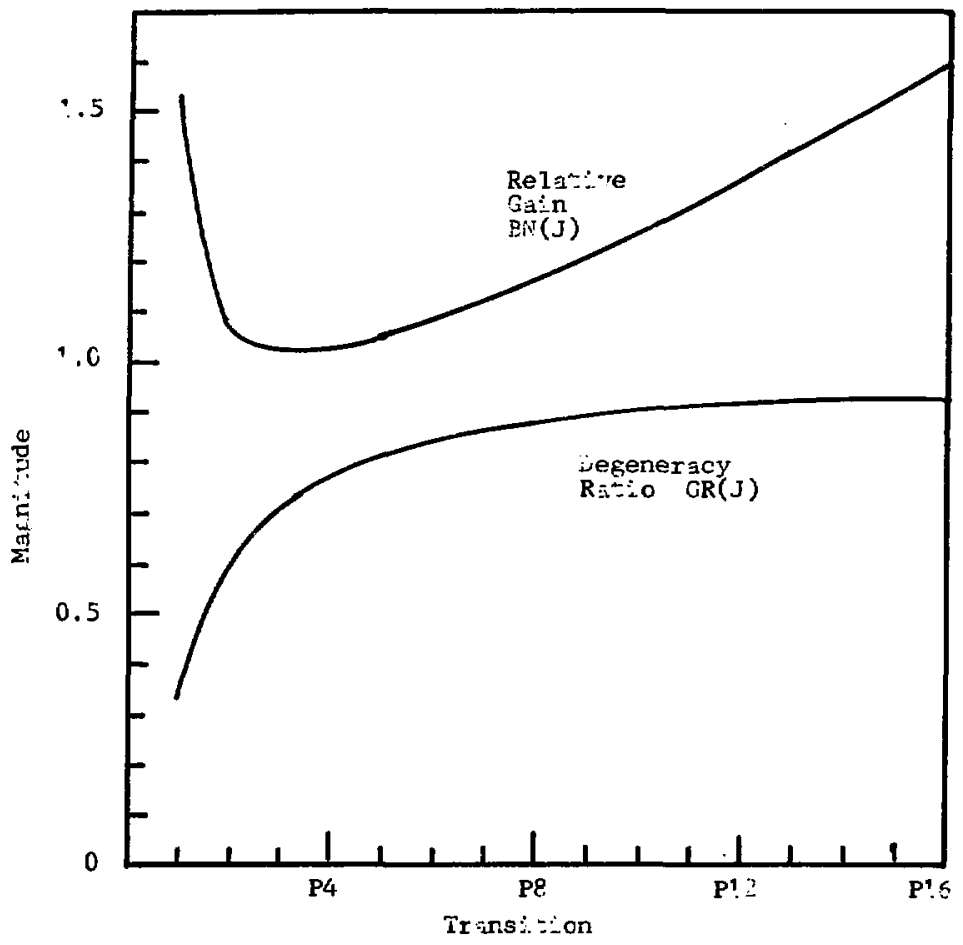

Fig. 7.5 egenericy $r: t: 0$ and rel:t:re gain for P-branch trans:tions. 
8. CALCULATED WAVEFORMS AND DETAILS OF LASER OPERATION

\subsection{Introduction}

The IXION code, described in the previous section, has been used to calculate laser waveforms which could be compared with experiment. The relevant experiments are described in Section 3 . The agreement between experiment and calculated waveforms is quite good. As a result we can consider that the code uses a fairly accurate model of the physics involved. Assuming that the model is correct we can increase our understanding of the operation of the pulsed HF laser. We will also examine the assumption of rotational equilibrium used in earlier codes, and show how the waveforms calculated by the IXION code are superior to those calculated with simpler models.

\subsection{The Nominal Calculated Waveforms}

The "nominal" calculated waveforms are shown in Fig. 8.1. The values of the parameters used attempt to match experimental values. The most important parameters are the total pressure of 15 torr with the ratio of $\mathrm{NF}_{3}$ to $\mathrm{H}_{2}$ equal to 5.2 , the density of $\mathrm{F}$ atoms which was $1.25 \%$ of the initial desnity of $\mathrm{NF}_{3}$, and the peak rotational relaxation rate which was about five times gas kinetic. These values of the parameters were chosen as the noninal case because they seemed to be the best fit after running a number of cases. The judgment of best fit was made after visual compar- 
ison, so is partially subjective. A quantitative fit, in this case, would only hide subjective judgments as to the relative importance of the features to be fit.

The calculated data will generally be displayed in the form of Fig. 8.1. The total power waveform will be enclosed in a box in the lower right hand corner. The full scale magnitudes of the time and power axis are given. The time scale is the same for the individual waveforms, but full scale power for the individual waveforms is $1 / 4$ that for the total power. If a spike is more than $110 \%$ of full scale, it is truncated at $110 \%$. The power scale is adjusted for each run so that the total power spikes will be at least half of full scale, but not greater than full scale. In addition to the waveforms, a table was calculated showing the fraction of the total energy emitted on each transition. This will be shown in tabular form where it is appropriate. As a further aid in comparing calculated results with experiment, Fig. 8.2 shows the calculated results smoothed by a 30 nanosecond detector response time. The full scale power is half that of Fig. 8.1 because the smoothing reduced the height of the spikes

Comparison of calculated and experimental total power plots show's many similarities. The first spike in both cas'as is the V2-1 transitions beginning to lase. These are the first because they have the largest inversion as well as twice the dipole matrix elemen of thr v1-0 transitions. 
The second spike in the calculated results is the lasing on v3-2. The major spike occurs when V1-0 transitions begin to lase. At that point cascading begins and all four vibrational levels interact. Experimentally, the v3-2 and V1-0 transitions appear to start about the same time. Calculations at different vibrational rates have shown that this relative timing is fortuitous. Depending on the magnitude of the V3-2 inversions, they may begin to lase before, after or simultaneously with the V1-0 transitions.

The bumps and wiggles after the main spike in the calculated waveform are due to damped relavation oscillation and a phenomenon similar to "J-shifting," where one transition suddenly drops a lot of power and another picks it up. This will be discussed lat $^{\text {? }} \mathrm{r}$.

The calculated total energy is $2.40 \mathrm{~mJ} / \mathrm{cm}^{2}$. The dissociation was adjusted to agree with the experimental .9 to $1.0 \mathrm{~mJ}$ over a $0.4 \mathrm{~cm}^{2}$ beam area.

The pulsewidths of the inc.vidual calculated and experimental waveforms show the same trend: the pulsewidths increase from $\mathrm{P} 3$ to $\mathrm{P} 6$, and agree well in magnitude. The P7 transition is somewhat anomalous.

A11 of the P3 waveforms are rather narrow spikes. The calculated V2-1 P3 is a double spike, but the experimental trace shows only one (Fig, 3,2). An examination of the experimenters notebook showed three V2-1 P3 waveforms. Two had a single spike and one a double spike. The difference 
depended on the time between the first spike and the major spike. The P3 transitions stop lasing because the inversion disappears. If it disappears before the major spike there will be only one P3 spike. The calculation shows a large V3-2 P3 spike, not shown with the experimental waveforms. An examination of the notebooks shows a probable weak lasing at just about the sensitivity limit of the detector. Some calculations with high vibrational relaxation rates have greatly reciuced the $\mathrm{V}=3$ population and reduced the P3 lasing. Even higher relaxation rates completely suppress al1 13-2 lasing. The calculated relative heights of the V2-1 P3 and V1-0 P3 are not in agreement with experiment. The height of the V1-0 P3 pulse depends on the detailed chemical pumping rates which were estimated for $y=0$, due to lack of data, and the experimental heights may not be too accurate. The laser has some problems in reproducibility and the attenuators used with the detector were changed from time to time. As a result, pulse heights have to be compared to other transitions measured under similar conditions. The height of the P3 line may be as low as that of the P6 or it may be as high as shown.

The calculated P4 waveforms agree well with the experimental, except that the calculated v3-2 P4 waveform has a longer duration than the experimental. Both calculated and experimental waveforms show an initial spike and a later, weaker spike, or at least a hump. The laboratory notebook shows sone variation on the $\mathrm{V} 2-1 \mathrm{P} 4$. In some cases the first 
spike is hightr than the second, in other cases they are equal, and sometimes the second spike is higher. The calculated waveform is within the limits of experimental variation. The calculated V3-2 P4 pulse is too wide to match experiment. Calculations indicate that this duration is due to a relatively large V3-2 inversion. Mechanisms which would deplete this inversion would probably reduce the pulsewidth. The V3-2 P4 pulse is terminated when the P5 reaches higher gair. and the $V=3$ populations then feed the P5 rather than the P4 transitions. This is similar to "J-shifting." True "J-shifting" occurs only if there is rotational equilibrium in each vibrational level (Chester, 1970; Emanuel, 1971). W'e will also call the phenomenon seen here "J-shifting" in the absence of a better term.

The calculated and experimental V2-1 P5 waveforms agree in height, the number of spikes and duration. The experimental V1-0 P5 is missing due to water vapor absorption. Experimentally the V3-2 P5 was missing, although there is some indication of weak lasing at about the same time the P4 turns on. If the V3 population were depleted early the P5 would not appear at late times as shown in the calculated wave form.

The P6 and P7 transitions are the least reproducible experimentally, and are most sensitive to parameter changes in the calculated results. This will be seen later when more calculations are presented. The calculated V1-0 P6 and $\mathrm{P} 7$ waveforms are another example of "J-shifting." The 
P7 output goes up at the point that P6 goes down. A closer examination shows that not only these two levels are "J-shifting" but two complete cascades. The first cascade is V3-2 P4, V2-1 P5, and V1-0 P6. The second cascade is V3-2 P5, V2-1 P6 and V1-0 P7. At about 1.4 microseconds, the entire first cascade gets weaker and the second gets stronger. This shows that cascades are relevant to the operation of the laser, but that their effect is diluted by the rotational relaxation. This will be discussed in more detail later.

The experimental waveforms show mixed evidence of the importance of cascades. The "J-shifting" is evident as we look down the V2-1 column of Fig. 3,2. As each transition goes out, the one below it has a hurip or a spike. The ev1dence for cascades, however, is weak or contradictory. Al1 of the transitions in the first cascade described above are present, but the waveforms appear to be rather independent. The second cascade lacks a V3-2 transition. If the cascade were strong we rould see a late hump in the V1-0 P7 corresponding to that in the V2-1 P6. This hump is definitely missing. The hump in the P6 transitions is not very reproducible, so the experimental waveforms may be misleading. It is also possible that certain approximations in the calculations distort the results. In particular, the calculations assumed a constant temperature of $300 \mathrm{~K}$, and that rotational relaxation occurred by single quantum jumps. Neither of these is completely justifiable physically. 
Nej.ther the calculations nor experiments showed lasing on P2 transitions. Calculations showed weak lasing on V2-1 P7 to P14, and also on V1-0 PE to P14. The amplitudes, however, wfre $30 \%$ of V2-1 P6, or less, and decreased to a few weak, isolated spikes at P14. If these transitions did in fact lase as calculated, the intensity was too low to distinpuish them experinentally from the noise. Transitions up to Pl 7 have ben seen experimentally in a similar laser operatirg with lower threshold inversions (Pommer and Kompa, 1972).

The calculated fraction of the total laser energy emitted on each transition is compared with experiment in lable 3.1. Since experimental values were obtained by graphiwil intepration of waveforms, the thirc colum gives a better comparison. To obtain the third column the calculated values were sumned over the experimentally measured transitions. The calculated values were then renormalized by dividing by this sum. Exact numerical agreement is not obtained, but Ecneral trends are the same. Comparing these energies gives less information than comparing waveforms.

In summary, very good general agreement between calculation and experiment has been obtained with only one fully adjustable parameter, the fraction of $\mathrm{NF}_{3}$ dissociated. The fraction dissociated was adjusted to give about 2.5 milli.joule $/ \mathrm{cm}^{2}$ output lo correspond to an experimental output of about $1 \mathrm{mi} 11 \mathrm{i}$ joule in $0.4 \mathrm{~cm}^{2}$. The fractional dissnciation, $1.25 \%$, was in agreement with Saha equjlibrium calculations of Surry (1972). The rotational relaxation rate was adjusted 


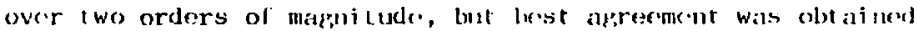
when the rotational relitxat ion rald was a hall of the 1 ine broadenjng, collisjon rate, or celual to it. This is conparalle t.o the experiment al 1 y est imated rotat ional retaxist i on rate for HCl (Polanyi and hoodall, 1972a). This is also the mosil roasonable physical estimate because: line broadening is caused by collisions which either change the rotational state or perturb the phase of the rotating molecule by an angle on the order of $\pi$ radians. A large fraction of line hroatening collisions should involve a change of rotational state. Other parameters were set at the most $1 \mathrm{ike} 1 \mathrm{y}$ value, based on known data or physical reasoning. The differences hetween the experimental and calculated results have been pointed nut, but the overall agreement is surprisingly good. Because of the good agreement between theory and experinent, we can have some confidence that the model is a reasonable representation of reality. The next section will describe in some detail the operation of the laser. The model drscribes the dynamics of variables which were not accessible experimentally. Therefore, we can use the model to increase our understanding of the relevant physical processes, When the description is finished, various parameters in the model will be varied and the results examined. 


\subsection{Sequence of Events During the Laser Pulse}

\subsubsection{Jntroduction}

fic can use the results of calculations with our model to understand what happens inside the laser. We can get intormation which is difficult to obcain experimentally such in the value of the populations and inversions. It might be possib1e to measure these variables experimentally, but it would li very difficill. On the other hand, these data come alnost autonatically from the code. The present model also gives considerably more detail than earlier models because rolational states are troated separately and the laser dunamics is treated by semi-classical equations. Thus the intormation used hore to Jepiet the operation of the laser is not aval lable elsewhere.

To illustrale the scquence of evernts we will use single Irines lrom a computer penerated nisvie (Creighton, 1974). lho. movie was generated irom nutput data of the $[x]$ oN code using the set of parameters designated as the nominal case at i5 Torr. Each frame of the movic shows the population of each vibrationit-rotational state, and the inteusity of any laser transitions connecting it to nthrer states. The total power wavelorm, up to the time which the frank represents, is shown in the npper ripht-hand corner. (Sed lip. 8.3.) the populalions ol all statos with the siture vibrational quantum mambers are sommetrod by strajght lines. The horizontal axis is the rolationil qualltum unber, i, The populations 
in the movie are distorted, except for $v=0$, in order to preserve inversions. This is necessary hecause the inversion is givern by

$$
N(v+1, . j-1)-N(v, . j) \times(2 . j-1) /(2 . J+1) .
$$

11 we plot the variables $N(v+1,1-1)$ and $N(v, J)$ we: niny have all inversiom even when $N(v+l, J-1)$ is less than $N(v, j)$. This creatos conlusion in trying to interpret the results. The inversion can be preserved if we display $\mathrm{N}(v, J) /(2.1+1)$ rather than $N(x, J)$, but this creates other distortions. The populat ions at high $J$ are already fairly small and if we divide them by 20 or 30 it is impossible to see their magnitude. Also the gain is rough1y proportional to the total number of molecules in a given state so dividing the population by $2 \mathrm{~J}+1$ gives $\mathrm{a}$ false impression of the realtive gains. A diflerent compronise was finally chosen. The bot ton state in each cascade is $\mathrm{N}\left(0, \mathrm{~J}_{0}\right)$. If we displaly $\mathrm{N}(\mathrm{v}, .1) \cdot\left(2 \mathrm{~J} 0^{+1}\right) /(2 \mathrm{~J}+1)$ we preserve inversions but with less distortion. The $V=0$ state has no distortion, and can be compared with the Boltzmann distribut; il (shown as a dotted $l i n e$ in Fig. 8.3). The low $J$ populations and gains are overestimated for other vibrational levels. The worst distortion is for $V=3, J=0$ where the population is a factor of 7 too high. It is much less for all other states. This distortion seems to be more tulerable than the other types.

When lasing occurs the I ransition is marked by a $1 i: c$ connecting the states involved. To show the intensity of the lasing an octagonal spiral is drawn at the center of the line 
(see Figs. 8.4 and 8.5). The number of straight line segments in the spiral is proportional to the logarithm of the intensity (over about 2 decades). The visual appearance is more nearly linear because the area of the spiral is proportioriai to the square of the number of segments.

$$
\begin{aligned}
& \text { 8.3.2 Initial Lasing or. V2-1 Transitions } \\
& \text { lie now proceed to discuss the sequence of events. }
\end{aligned}
$$

For the first 200 nanoseconds or so the populations build up, and rotational relaxation creates the double humped discributions seen in lig. 8.3. All or the V2-1 and V1-0 transitiot.s are inverted, as are the low J V3-2 transtions. Lasing first begins at V2-1 P9, rapidly followed by V2-1 P8. Figure 8.4 shows the population distributions at this point. The V2-1 transitions lase first because they are the most : crong,1: inverted. All V2-1 tranilions up to P15 have reached threshold inversion some time before Fig. 8.4, as have all v1-0 Lransicions up to Pl2. The radiation on all of these transilions has been increasing exponentially since they reached threshold inversion. The rimf constant for this increase: dependi on the magnitude of the inversion and the stimulated mpission cross section. The cross sections for a given vibrationa] love are al1 within a factor of two, and aro linesarly proportiomal to the vibrationtat guantum number of the lowere state.

The imarifmin roich throshold at different times for diflerent valursi of 1 , partly because the magnilude of the throshold inversion is a finction of I and partly because the 
stiates are pumped at. different rates. The threshold inversion is a function of $l$ inewidth and dipole matrix olement (Eu. 6.2\%). The: linrwidth decreases with .I for . I grodter 1 han 2 and asympLotically approaches the Doppler linewidth for J preiter thau 8(lig. 7.4). The threshold inversion is inversely proportional to the dipole matrix element, which increases at high l (Fig. 7.5). As a result the threshold inversion decreases monotonically with $J$, when $J$ is yreater than 3 .

The first laser transition to turn on in the present case is V2-1 P9. (In the movie "turn-on" is defined as reaching a level or $1 \%$ of the peak power of the highest spike.) Examination of the tabular output of the JXION code shows that mos $[$ of the $V 2-1$ transitions are increasing rapidly as py turns on. The P9 transition turns on first because it reached threshold before transitions of lower $J$, but it has a faster time constant than transitions of higher.] because of its anper population. However, other transitions turn on alnost inmediately as shown in Fig. 8.5. The sequence of appearance of V2-1 transitions is P9, P8, P10, P7, and P6, all shown in Fig. 8.5. These are followed by P5, P4, P11, P3, P12, and P13 as seen in Fig. 8.6.

The fact that $P 9$ turns on first is a function of the parameters chosen and some other transitions might turn on first if different parameters were chosen, particular-ly different values for linewidth and rotational reluxation rate. Experimentaliy, also, a different transition might turn on first, but the qualitative picturs of what occurs is still correct. 
lig gure 8.5 shows an example of hole burning in the rotational distribution. On the $\mathrm{V}=2$ level the $\mathrm{J} 7$ and $38 \mathrm{popu}-$ lations are depressed relative to others in that level. This loss of population has populated the $\mathrm{J} 8$ and $\mathrm{J} 9$ states in the $\mathrm{V}=1$ level. The hole burning occurs because the lasing changes the molecular states much faster than rotational relaxation can smooth the distribution. Additional hole burning can be seen in Fig. 8.6, but a short time later rotational relaxation has smoothed this out as shown in Fig. 8.7.

The relaxation oscillations discussed in Section 6 are also visible in this sequence of frames. In Fig. 8.4 all V2-1 transitions are inverted and the P8 and P9 transitions are starting to lase. In Fig. 8.5 the inversion has been lost on the P9 transition and is below threshold on P8. The intensity of radiation on these transitions is near its peak. Adjacent transitions are still increasing in intensity. Shortly after the peak of the first spike, Fig. 8.6, most of the V2-1 inversions are reversed and the $P 7, P 8$, and $P 9$ transitions have extinguished. The P4, P5, and P6 transitions are near their peak in intensicy and are responsible for the spike in the total power. The P3 transition is just now turning on, as is the P12, which will shortly be followed by P13. The 1ittle jog on thr lrading erlge of the cotal power curve occurred when the $P 8$ and $p 9$ transitions were getting weaker due to loss of inversion and the other transitions had not. yet reached a high intensity. 
It is interesting to note what has happonorl to populations of the vibrational levels in the period betwren Figs. 8.4 and 8.7. The poptlat inn of the $V=3$ level hat: inereased about $50 \%$ due to chemicial punping with a small losst due to vibrational relaxation. Jhe population of the $v=$ ? level. has remained about the sames because it has 10 t populaLion to the $V=1$ lovel which now has about the sane populat:on as $V=2$. he will see later that at steady-state the population of all vibrational levels is about. the same. The $V=0$ level, like the $V=3$ level, has increased about $50 \%$ in population. The population distributions of f'ig. 8.7 are $3 t i ? 1$ a long, ways from a Boltzmann distribution, but rotational relixidion has increased the population at low $\mathrm{J}$. The $\mathrm{V}=1$ level has a larger population at high $J$ than $V=2$. This is causer by the selection rules for lasint which causs an increase of one in the rotational quantum number. This difference will remain until lasing, occurs on the 1-0 transitions. Further insight into the processes we have been considering can he obtained by looking at the time history of the laser powers, inversions, and populations of one cascade. Figure 8.8 sl Jws these variables for the cascade consisting of the states V3.33, V2.34, V1J5, v0J6, and the transitions that couple them. (In this rigure the populations are not (listorted.) In Fig: 8.8b we see the V2-1 inversion rising jnitially (curve labeled 1). At about 100 nanoseconds, it reaches threshold inversion. (In lig. 8.8 the inversions at 1 microsecoud equal threshold inversion.) Al about 250 nano- 
seconds the inversion befins to drop rapidly. Comparison with Fifr. 8.8a and 8.8e shows that the drop occurs because of lasinf, and that the population of $v 2.14$ is decreasing and V1.J5 is increasing. The changes in population are nearly mirror inizes at this point. Jropping the population of V2 .34 increases the V3-2 inversion and increasing the VI J5 population increasos the Vi-O inversion. (The time where the V2-1 inversion starts to drop corresponds to a time just after that of Fig. 8.5; and the minimum V2-1 inversion (orresponds roughly to $F i g .3 .6$.$) Atter the population of$ V 34 roaches minimum it 1 pidly restored to equilibrium whith adjacent states by rotational relaxation. As it approiches equilibrium, its population continues to increase due to chemical pumping but at a slower rate. A similar thing happens to the V1PJ population except that lasing leaves it overpopulated and rotational relaxation decreases the population. At about 300 nanosecomms v1p5 is near cqui $i \mathrm{jbrim}$ and its population has bogun to increase dlue: to (hemical mupin?. At aboul that time arlditional processes become importiul becaluse V3-2 P4 begins to lase.

\subsubsection{Initial Lasing on V3-2 Transitiom}

Some V3-2 1 rinstitom were inverted from the beginning, but at showll in lifi. 8.4 the inversions woro much smaller than

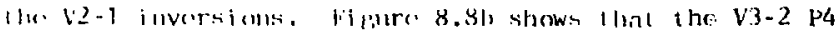

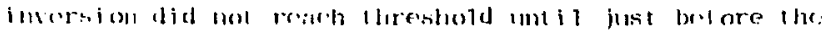

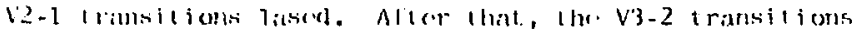

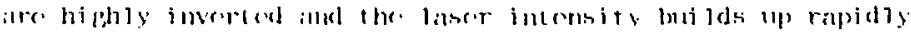




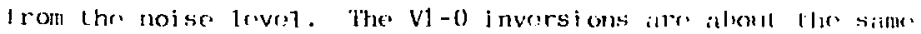
arder of magnitudr at 300 namosecomds and the cormesponding; laser intensitios are also building up, but the dipolo matrix elements for V3-2 are 3 times those for VI-0, so I he bui ldup on V1-0 is slower than V3-2. At alout 320 nanoseconds V3-2 $P 3$ and $P 4$ begin to lase as slown in fig. 8.7. The $V=3$ populations now drop and the $V=2$ populations rise. The $V=1$ level is still coupled to $V=2$ by $V 2-1$ lasing trantition so the $V=1$ populations also rise unti] $V=3, V=2$, and $V=1$ populations are all nearly equal as shown in ligs, 8.9 and 8.10. This significantly increases the $V 1-0$ inversions as shown in l:i $g .8 .8 \mathrm{~b}$.

Another feature is that lasing on the V3-2 transitions now pumps the V2-1 transitions. This is seen in Iig. 8.8a where the V2-1 P5 power tends to follow the V3- 2 P4 power with a slight lag in tjme. This can also be seen by comparing Fig. 8.7 with 8.9 and 8.10 . The V2-1 P5 power increases a great deal from the level of Fig. 8.7. The erlect is tess pronounced with V2-1 P4. Figur: 8.9 also shows that the V3- 2 P3 inversion is nearly zero at the peak of the pulse. Figure $8.8 \mathrm{~b}$ shows that, unl1ke the V2-1 transtions, the inversion doesn't reverse but rapidly settles down to steady state.

The timing of the V3- 2 lasing relative to the V2-1 is dependent on the parameters used in the model. It should always occur after the V2- 1 lastng because $V=2$ is more 
strongly pumped than $V=3$. However, the V3-2 inversion may be weaker than the V1-0 inversion, depending on the populations of the $\mathrm{V}=3$ level. In particular, $\varepsilon$ weaker pumping or faster vibrational relaxation could reduce the population at $\mathrm{V}=3$. Experimentally it appears that the v3-2 lasing begins about the same time as $\mathrm{Vi}-0$.

\subsubsection{Initial Lasing on V1-0 Transitions and Steady State}

As noted above, lasing on v3-2 transitions increases the V1-0 inversions by increasing the populations of the $V=2$ and $V=1$ levels. At about 370 nanoseconds, the $V 1-0$ transitions turn on. The sequence is similar to the V2-1 sequence, with some differences. The V1-0 sequence is P10, P9, P11, P5, P4, P6, P3, P8, P7, and P12. Figure 8.10 shows the populations and intensities at the instant that P4 turns on. It is interesting to note that V2-1 P3 through P7 are lasing, though P7 is weak and higher transitions are not lasing. Figure 8.11 shows the situation at the peak of the total power curve. All of the VI-0 transitions have turned on and the inversions have already reversed on $\mathrm{P} 4$ and $\mathrm{P} 5$. Also the V2-1 P7 transition is lasing strongly as are the P8 and P9 transitions. This occurs because depleting the population of $V=1$ pumps the V2-1 transitions just as augmenting the $V=2$ populations would. The V2-1 P14 transition has also turned on, but appears to be less directly connected with pumping due to depletion of the $V=1$ leve1. The close coupling between levels can also be seen in Fig. 8.8a where all three transitions contribute to the 
largest spike. After the spike there are some small relaxation oscillations in the populations (Fig. 8.8c) after which the laser settles down to a steady state behavior as showr in Fig. 8.12. One of the notable features is the presence of lasing on all three transitions of two cascades. Simultaneously, however, there are cascades with only a single transition lasing. This shows that the operation of the laser cannot be characterized entirely in terms of the behavior of vibrational levels in equilibrium, but neither can it be characterized entirely in terms of cascades. The rotational relaxation and lasing are interacting in a more complex manner.

Steady state is characterized by the inversions for the relevant cascade being constant. Figure $8.8 \mathrm{~b}$ shows that this begins shortly after the largest spike and continues for some time. Figure 8.12 shows all except a couple of high $\mathrm{J}$ inversions at steady state. However, the total population with $V=0$ exceeds that of $V=1$, which exceeds $V=2$, which exceeds $\mathrm{V}=3$. This is the situation called "partial inversion" in the 1iterature. The actual transitions are inverted but the total populations in the vibrational levels are not inverted. In contrast, the situation in Fig. 8.4 represents a "total inversion." The concept of "partial inversion" is useful when considering the entire vibrational level, but has less meaning with the present model: So long as the lasing continues at steady state, the population curves will remain close to each other, and will increase together in population. 
The P1 and P2 transitions do not lase at any time. The reason for this can be seen by examining Fig. 8.13, which shows the poprulations and inversions for V2-1 P1 and V1-0 P2. Initially the V2-1 inversion is increasing but about the time it reaches threshold it is reduced because of a rapid buildup in the $V=1$ population. This buildup is quite different than the V1J5 buildup in Fig. 8.8c. There, the buildup was nearly j.nstantaneous due to lasing. In the case of the P1 transition the buildup is due to rotational relaxation from states which were lasing.

The P5 transition turns on some 30 nanoseconds before the Pl inversion begins to disappear. When we examine the inversion for V2-1 P3 (Fig. 8.14b) we see that the P1 inversion begins to change a few nanoseconds after $P 3$ turns on; and that the $P$ ? inversion is also strongly affected by rotational relaxation, as shown by the flattened top on the inversion curve and the change of slope after lasing begins.

The increase in the $\mathrm{V}=1$ population creates an inversion on V1-0 P2 that is well above threshold. This would readily Jase if given time, but lasing on other V1-0 transitions depletes the $P 2$ transition by building $u p \quad V=0$ and depleting $V=1$. This is further confirmed by examining the movie frames from lig. 8.5 to 8.12 .

\subsubsection{J-Shilting}

lijuro 8.12 shows both V3-2 P3 arid P4 lasing. later than P3 transilion reases to 1.ase because the inversion is lost as shown in lip. 8.15. At about 1.35 microseconds the 
V3-2 pj trunsition turns on ats :nown in lips. 8.16 atud 8.17 .

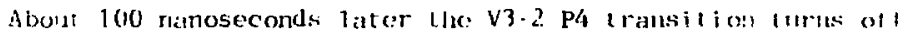
ats shown in figs, 8.18 and 8.8 . There is lict to change in the lasing, after this point except for al gradulat roduction in laser power as shown in fify. 8.19. This is similin to .1.shithing as described by chester (1970) and Emanul (1971). The mechanism by which this occurs can be seen b. exantining $l i g .8 .17$. The v3-2 P5 transition is inverted whon the V2-1 cransitions lase but is depleted before mm-on wh"?: the adjarent V3-2 F4 lises. For sume time after that, the 25 inversion is posicive but below throshold. Thre inversion continucs to increase, reaching threshold at 0.9 microseconds, dis: ro bui lohp in the 23.34 population. At that point, a slow buildup of the laser radiation begins, with turn-on occurring about $1.35 \mathrm{microseconds.} \mathrm{The} \mathrm{inversion} \mathrm{then} \mathrm{trops} \mathrm{to} \mathrm{thresh-}$ old and rotational relaxation pulls the V3-2 P4 inversion helow threshold, causing that transition to turu of $f$ (ser Fig. $8.8)$.

This J-shifting has occurred at constant temperature. The possibility of this has been noted before (Enanuel, 1971). The J-shirting would te erharced by a rising temperature which would cause the peak of the rotational distribution to shift $[0$ higher $J$ as cime increased. The phenomenon of $J$-shifting under the assumption of rotatiora] equilibrium has been discussed extensively by Emanuel (1971). We will do a brief calculation to see if present results are in agreenent with equi?ibri.um theory. 
At equilibrium, the populations of two adjacent rotational states are given by

$$
\frac{N_{J}}{2 J+1}=e^{-2 \theta J} \frac{N_{J-1}}{2 J-i}
$$

where $\theta=B_{0} / R T$ and $B_{o}$ is tine rigid-rotor spectroscopic constant. For $\mathrm{HF}, \mathrm{B}_{\mathrm{O}}$ is $21 \mathrm{~cm}^{-1}$ so $\theta=0.1$ at $300 \mathrm{~K}$. Let the $\mathrm{v} 3-2 \mathrm{P} 4$ inversion be constant at a value $G_{1}$, then

$$
\mathrm{N}_{3,3}-\frac{7}{9} \mathrm{~N}_{2,4}=\mathrm{G}_{1} \text {, where } \mathrm{N}_{2,4} \text { is the population }
$$

of the state $v=2, J=4$.

There is an additional constraint due to pumpine that $N_{3,3}+N_{2,4}=f(t)$, where $r(t)$ is a pumping function. Normally $f(t)$ is monoconicaliy increasing, although this is not necessary. At Bolt\%mann cquilibrium, the populations for the v3-2 P5 transition are

$$
\begin{aligned}
& N_{3,4}=\frac{9}{7} N_{3,3} e^{-8 \theta} \\
& N_{2,5}=\frac{11}{9} N_{2,4} e^{-100} \text { and the inversion is } \\
& G_{2}=N_{3,4}-\frac{9}{11} N_{2,5} .
\end{aligned}
$$

lihen we solve those equations for $G_{?}$, we get

$$
G_{2}=0.625 G_{1}+.046 f(t)
$$

From Fi.g. 8.8 we lind $G_{1}=1.3 \times 10^{-11}$ and $N_{2,4}=9.3 \times 10^{-11}$ at 1.0 nicrosecond, resulting; in a value of $6.4 \mathrm{G}_{1}$ for $f(t)$. This gives

$$
\mathrm{G}_{2}=.917 \mathrm{G}_{1} \text { at } 1 \mathrm{mij} \text { crosecond, }
$$

which is an juversion of $1.1 \times 10^{-11}$. This agrees with the 


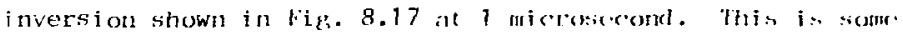
what higher than the throshoid inversion for the liz-2 55 triatsition $\left(.7 \times 10^{-11}\right)$. Theretore, then pr lases it pulls the $P_{4}^{4}$ inversion below chrorshold.

if wo hed troated the laser in a stoidy state approxillation, the results wolld hide been slighl ly ditferent As snon as the P5 inversion reachrd theshold lasing. would have swiched to that transition and P4 woldd have been Extinguished (Emanue1, 1971; Airry, 1970). liith the prosem model, there was considerable delay after threshold was reached before the laser intensity built up, and both lased simultaneous 1 y for a while.

The mixture of cascade and equilibrium brhavior is again illustrated in Figs. 3.8 and 8.17 . lihen thr v3-2 P4 turns off, the power is redured in the lower steps of the cascade. The power represented by pumping into thr $\mathrm{V}=3$ level is ransferred to the next rascade. Thus, we see that the cascadrus are important, yet power could not he transierred from one to the other unless the $V=3$ level bchaves as a leve: which is nearly in equilibrium.

The non-equilibrium nature of the present model is shown in $\mathrm{Fj} g .8 .17$, where the populations are nearly constant, rather than monotone increasing. At higher J-values the populations actually peak and then fall of $f$ as shown in Fig. 8.20. This occurs because the chemical pumping is initially very sirong and well out of equilibrium. As the chemical punping rates fall, the populations at high $J$ are reduced through rotational relaxation. 
8.4 How Valid is the Assumption of Rotational Equilibrium? Earlier modeis of the HF chemical laser have assumed rotational equilibrium within each vibrational level, and this equilibrium is usually taken to be at the translational temperature. The present model contains sufficient physical detail to see if the rotational equilibrium assumption is justified. We already know that the chemical pumping rate distribution is nowhere near the Boltzmann distribution at reasonable temperatures; we will want to see how rapidly this relaxes towards a Boltzmann distribution. We will also want to look at possible hole burning in the population distributions and to see if we can better understand J-shifting.

The population distribution at 41 nanoseconds is shown in Fig. 8.21. Comparison of Fig. 8.21 with the pumping rates (Fig. 7.2) shows that this distribution is mainly due to the pumping rates.

Somewhat later, at 156 nanoseconds, the population distributions develop a double hump as shown in Fig. 8.22. The high energy peak is nearly the same as Fig. 8.21, but an additional nearly-Boltzmann distribution is superposed on the pumping distribution. The $V=3$ distribution is very near to a Boltzmann distribution largely because the distribution of pumping rates for $V=3$ nearly coincides with the $300 \mathrm{~K}$ Boltzmann distribution.

Around 200 nanoseconds lasing begins and greatly distorts the distributions. However, near the end of the pulse the distribution should be smoothed out, and inight have a Boltzmann form. 
The population distributions at 2.0 microseconds are shown in Fig. 8.23. At fi.rst glance they appear to approximate a Boltzinann distribution, but closer examination shows that a11 but $V=3$ have a population larger than Boltzmann at the higher energies. The nature of this high energy tail is better seen on a semi-logarithmic plot (Fig. 8.24). he then see that although there are very few molecules in the tail, the population ratic between adjacent states is not given by a Bolizmann distribution. However, for rotational quantum number less than 5 or 6 the population distribution is given to good accuracy by the Boltzmann distribution.

It seems clear that the major cause for the high energy tail is the fact that these states are still being pumped, though weakly, by the chemical reaction. Although some of the populations are six orders of magnitude too large, the absolute populations are sma1l at high $\mathrm{J}$. The time between collisions in 10 to 50 nanoseconds, so if the pumping were turned off the populations would reach the correct order of magnitude in a microsecond or less.

It is possible to fit a line through the tail of the distribution and obtain an effective temperature for states with rotational energies greater than $1000 \mathrm{~cm}^{-1}$. This temperature would be in excess of $1000 \mathrm{~K}$, depending on the fit chosen. It is not clear that defining such a temperature for part of a distribution is meaningful.

It is clear that the assumption of rotational equilibrium within each vibrational level is incorrect up to the 
time that lasing begins. At the end of the pulse, it is a reasonable approximatj on for states with low J. States with hi.gh $J$ have very small populations, but still orders of Inagnitude larger than a Boltzmann distribution. Thus, we must conclude that the assumption of rotational equilibrium is valid only near the end of the laser pulse and then only with qualifications.

In addition to the non-Boltzmann character of the population distributions there is sone "hole burning" in the populations as seen in FiE. 8.11. This occurs because lasing depletes the upper state (and augments the lower state) faster chan rotalional relaxation can smooth out the distribution.

\subsection{Comparison of the 1 XION Code Results with Earlier Codes}

Having seen low the results of the IXION code compared with experiment, it is interesting to compare the results with rarlier codes. Figure 8.25 shows the total output power of the $\mathrm{HF}$ laser as calculated by the Aerospace Corporation's RESALE: cole (Rapagnani, 1973). This calculation was meant to simulate a laser with 20 Lorr total pressure, cluse to the conditions of ligs. 8.3 and 8.1 . The RESALE code assumes rotalional equilibrium and treats the lasjng in a steadyslate approximation. The grneral waveform is correct; $i . e$. an expoinential derily. The wavelorm contains less structure than the cxperimental wavelorm (lig. 3.3) and that calculated hy tho JXION coin (Fig. 8.1). The RESALE code dorsn't 


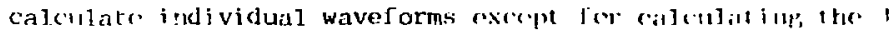
Válue of tha transition with maximum gain. This I value shifts from time to time, and the little l:litahes on the waveform of lig. 8.25 reprosent the tines when $1-s h i t$ ing. occur. If wavelorms were plotted by the RESALE rode: the' would be approximacely trapezoidal in shape because on transition turns $i{ }^{\circ}$ as the new ono turns on. Figure 8.25 atso shows the laser curning on very farty. 'Tho RESALl: model assumes rurn-on occurs as soon as the first transition roaches threshold inversion. It neglects the delay due to the finite time required to build up the laser intensity.

The IXION model differs from RESALE by treating rotational relaxation explicitly and by using semi-classical laser equations in a simplified form. The calculated results are similar except that the IXION code fives more detail and better agreement with experiment. The addition of rotational relaxation to the IXION code permits it to calculate simultaneous lasing on several P-branch transiiions. It also shows that cascade elfects are important along with J-shifting. Treating the lasing by semi-classical equations gives appropriate delays to turn-on and allows large jnversions to build up on certain transitions. It atso shows certain structure in the pulse.

This has been achieved with a single ad fustable parameter, the fraction of $\mathrm{NF}_{3}$ dissociated. Other parameters, such as rotational relaxation rate have been adjusted but best results are obtained when they are set to the most 1 ikely value based on physica? insight. 
The calculated rrisults of the $[X] O N$ model agree well with experiment. Becausc of this agreement we can place some confidence in the details of the code. The code calculates variables, such as inversions which are difficuic to measure experimental1y. By examining the code output we can gain greater insight into the operation of the laser. The IXION codr gives many more such details than the earlier codes. 

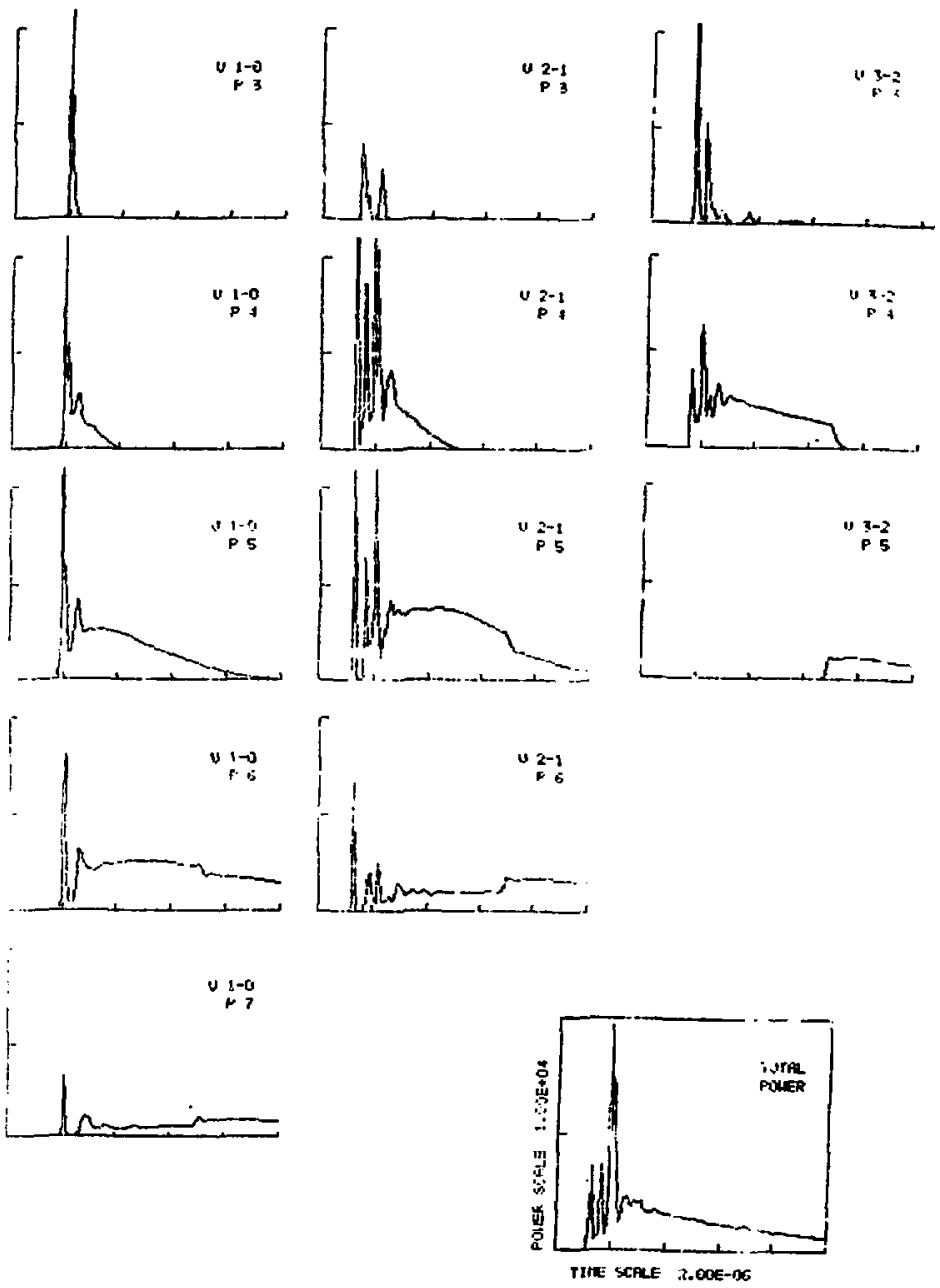

Fig. 8.1 Calculated waveforms at 15 Torr total pressure; $\mathrm{NF}_{3}: \mathrm{H}_{2}=5.2,1.25 \%$ of $\mathrm{NF}_{3}$ dissociated, rotational rate about $5 x$ gas kinetic. Total onergy $=2.40 \mathrm{~mJ}$. 

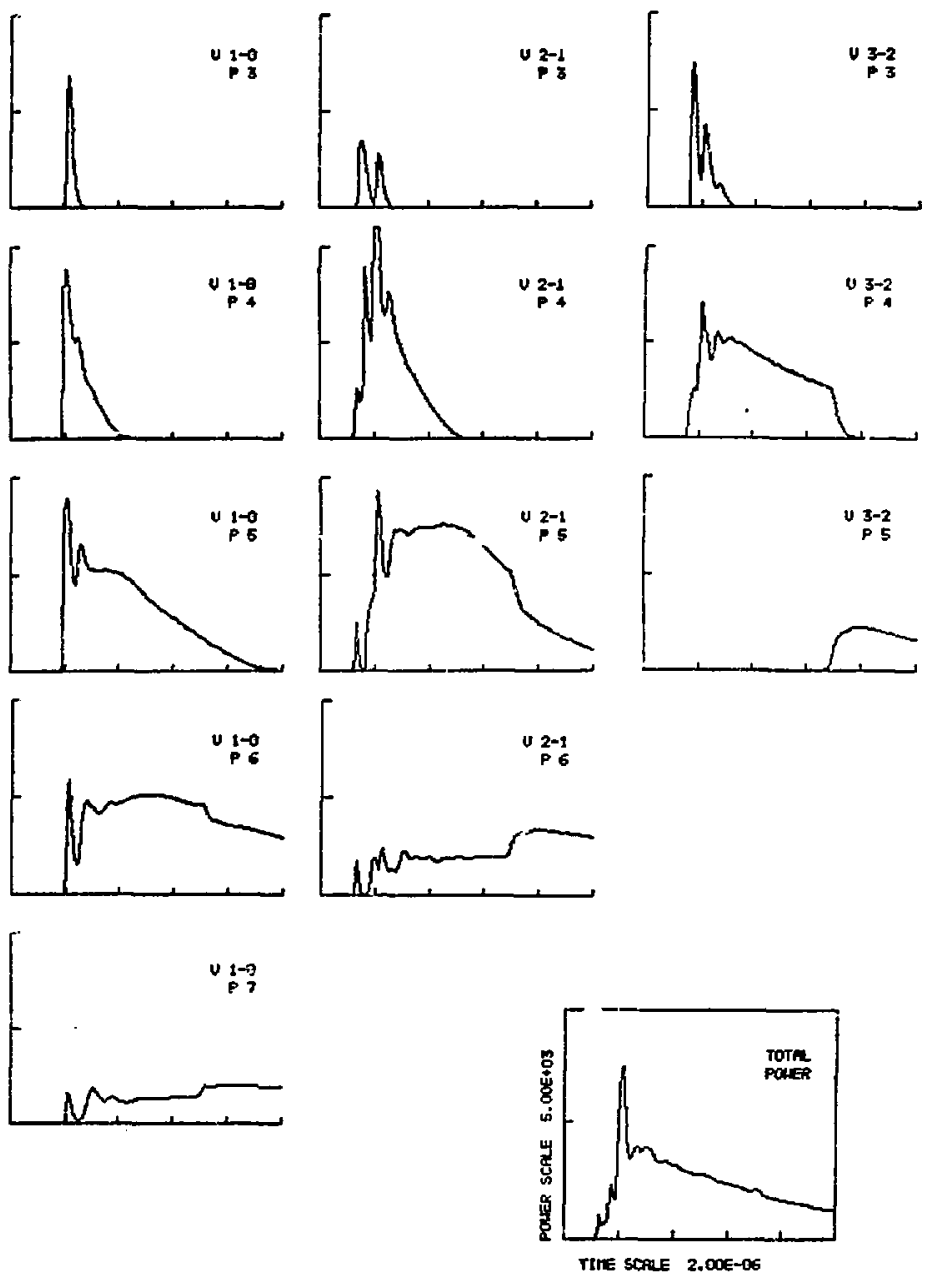

Fig. 8.2 Same waveforms as Fig. 8.1 except smoothed by a 30 ns. detector response. 


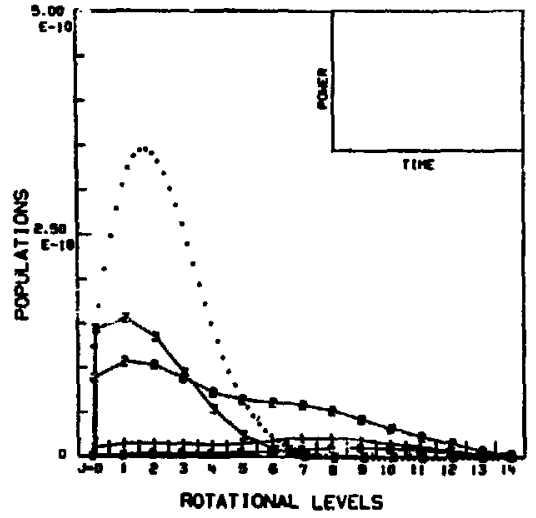

FiB. 8.3 Single frame from a movie showing popuiation distribution shortly before lasing begins.

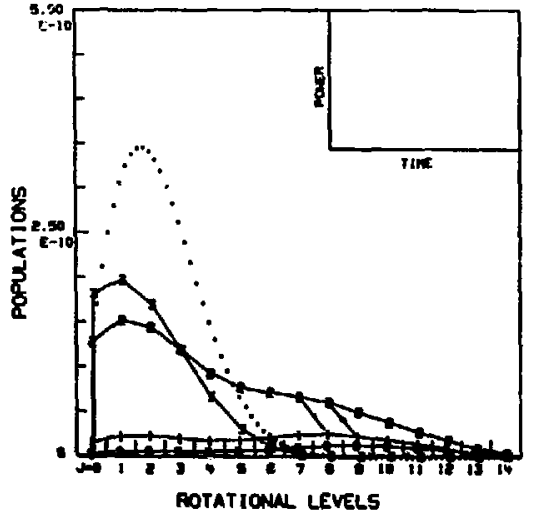

Fig. 8.4 Lasing begins on V2-1 transitions. 



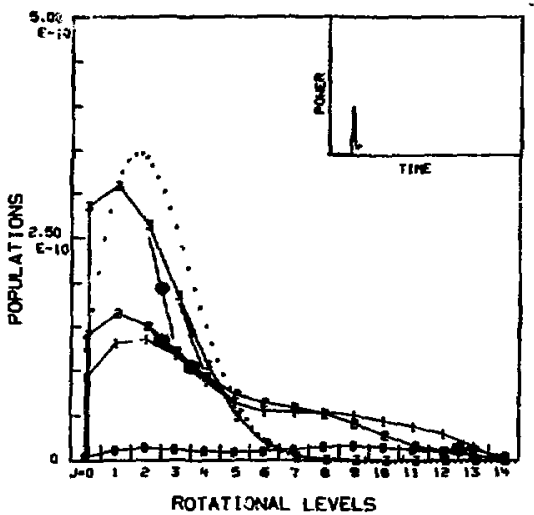

Fìg. 8.7 Lasing begins on v3-2 transitions. 


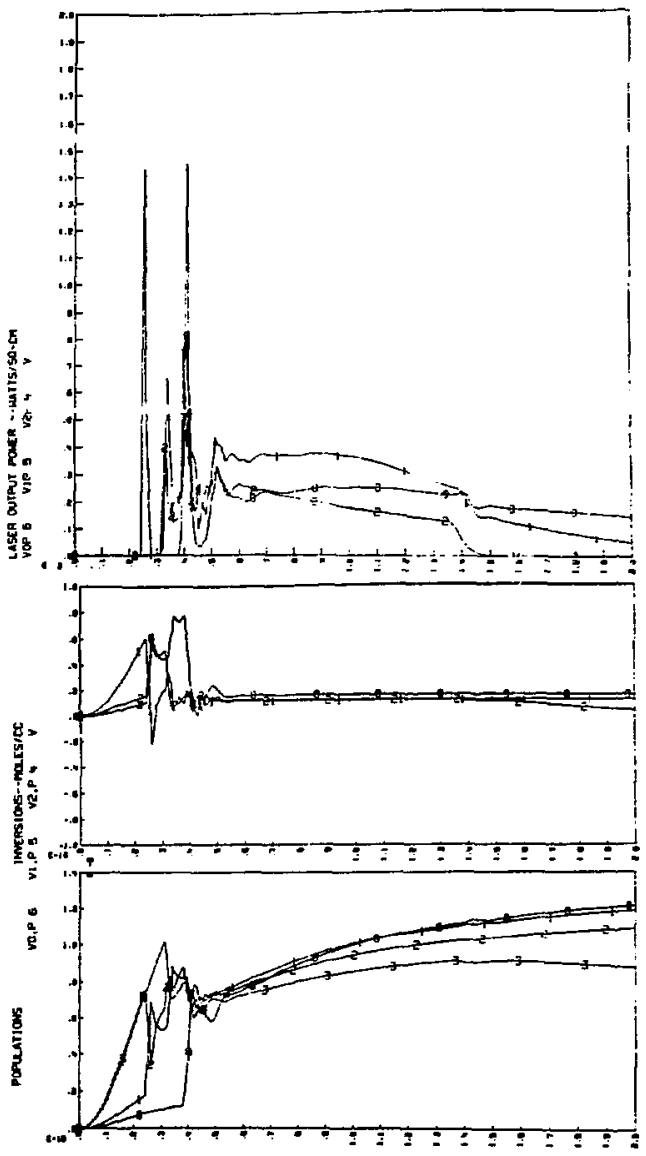

Time-microseconds

Fig. 8.8 Time history of the cascade consisting of V3-2 P4, V2-1 P5, and V1-0 P6. 


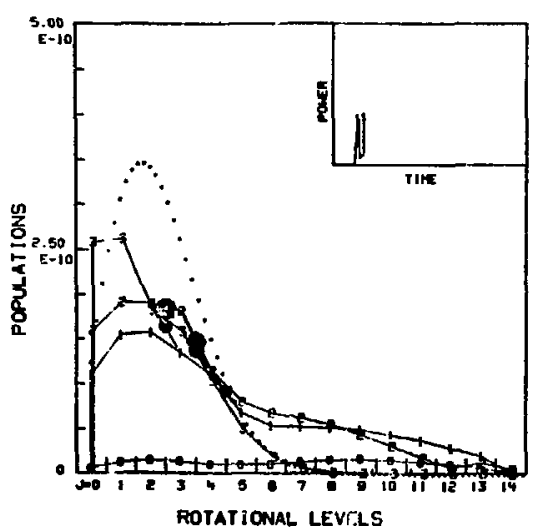

Fig. 8.9 Peak of the second spike.

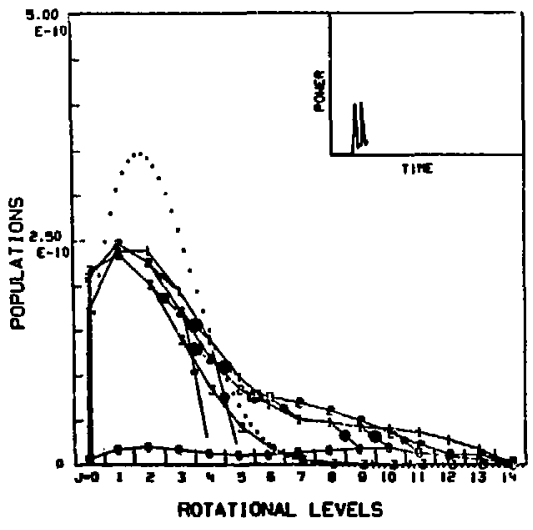

$$
\begin{aligned}
& \text { Fig. } 8.10 \text { Lasing begins on V1-0 } \\
& \text { transitions. }
\end{aligned}
$$

$\stackrel{\infty}{\infty}$ 


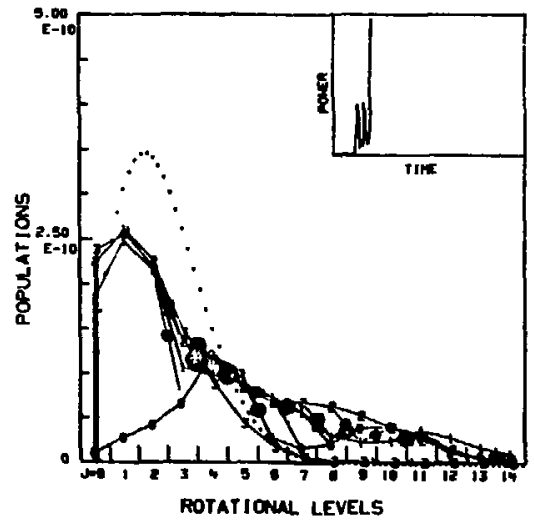

Fig. 8.11 Peak of the main spike.

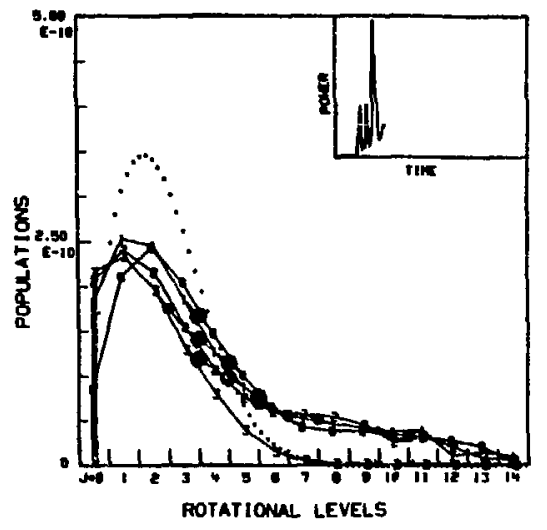

Fig. 8.12 The beginning of steady state operation. 


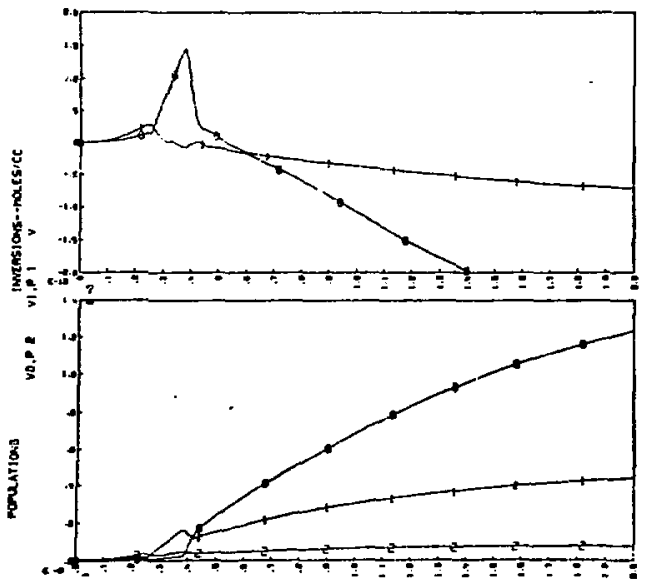

Time-microseconds

Fig. 8.13 Time history of the V2-1 P1, V1-0 P2 cascade. 

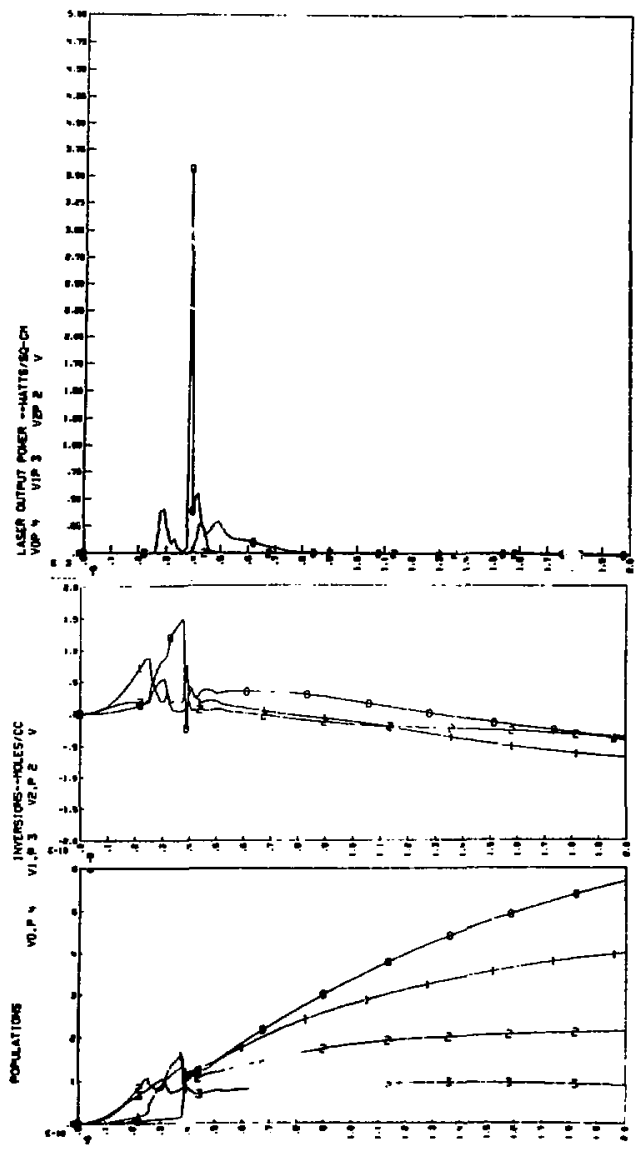

Time-microsec.

Fig. 8.14 Time history for the V3-2 P2, V2-1 P3, V1-0 P4 cascade. 

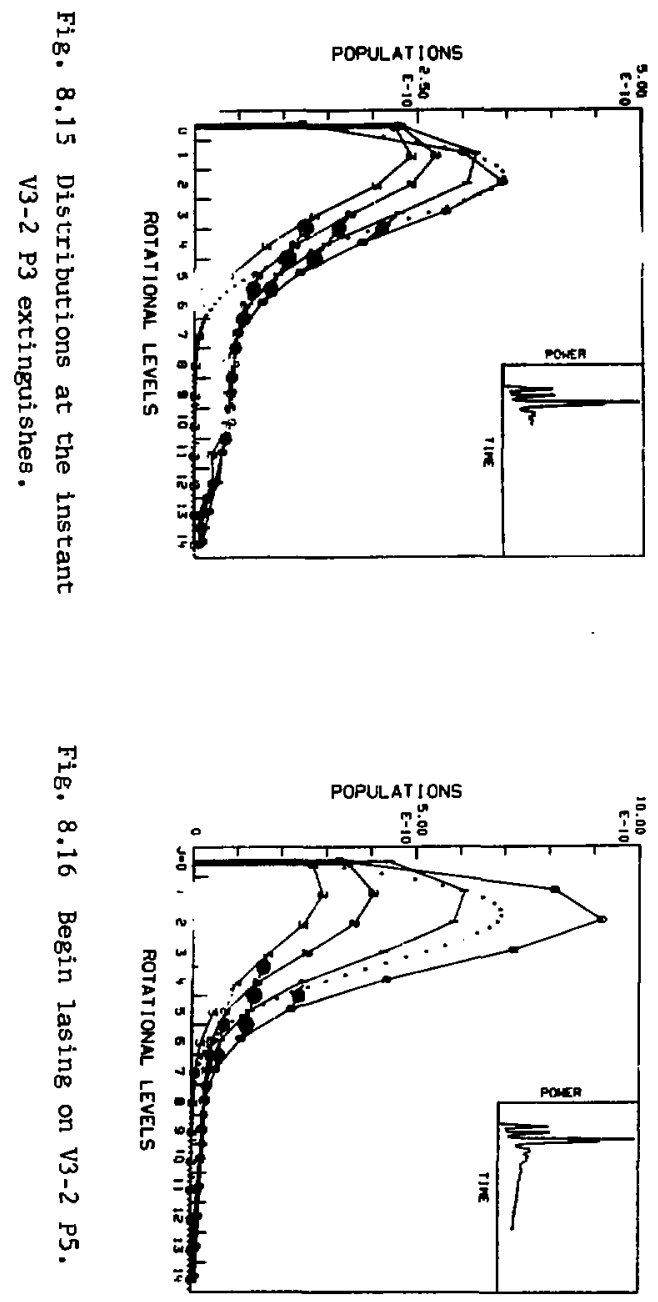


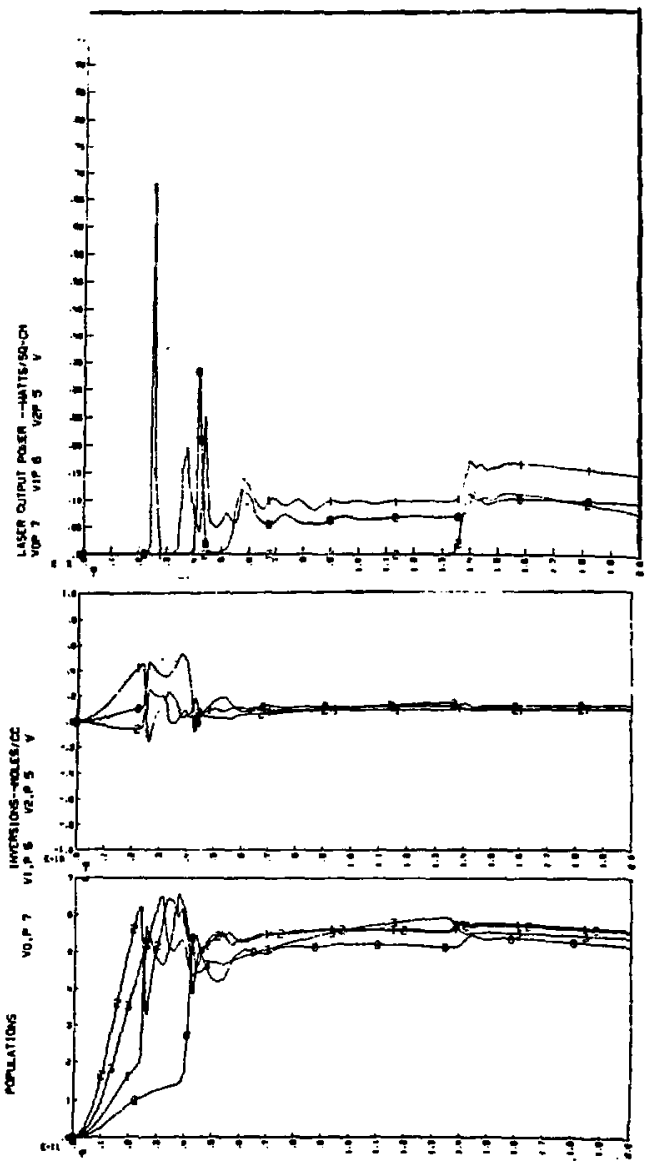

Time-ric. $25 e c$.

Fig. 8.17 Time history of the V3-2 P5, V2-1 P6, V1-0 P7 cascade. 


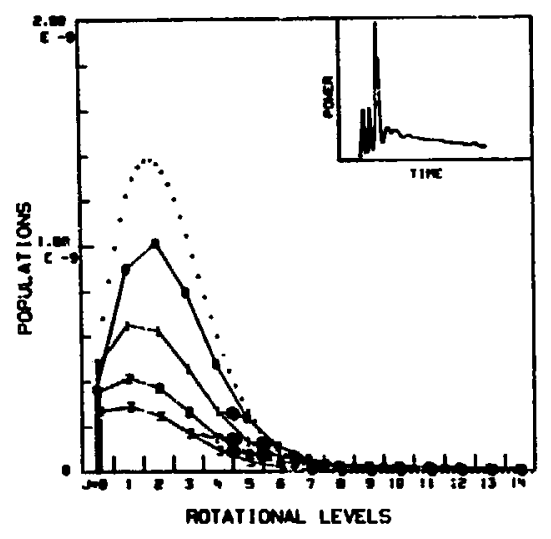

Fig. 8.18 Distributions when V3-2 P4 extinguishes.

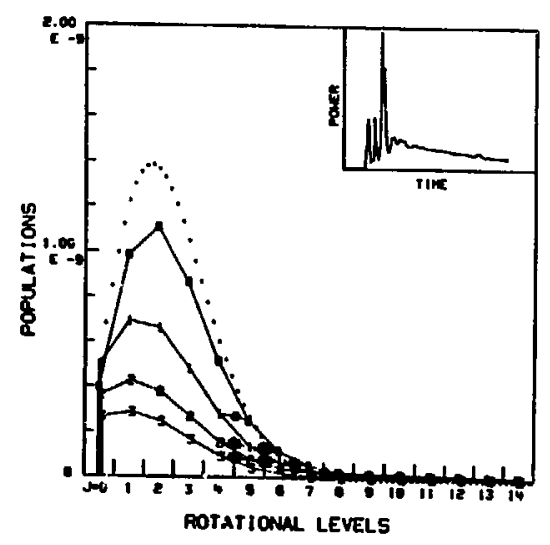

Fig. 8.19 Distributions near the end of the pulse. 

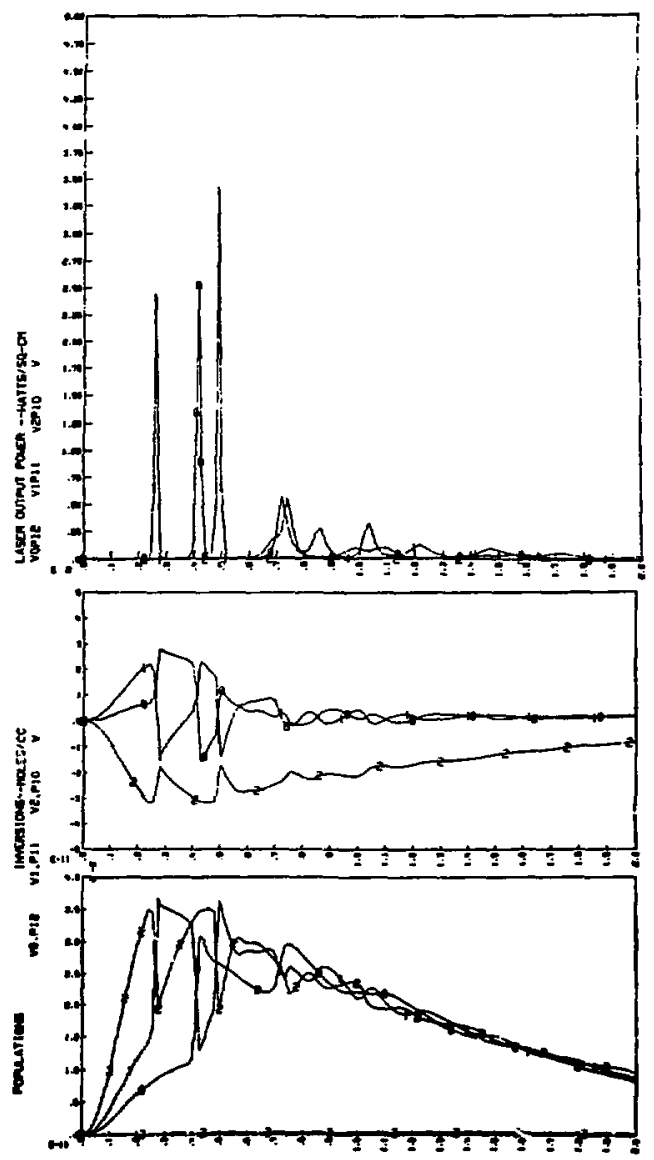

Time-microsec.

F18, 8,20 Time history of the V3-2 P10, V2-1 P1I: V1-0 P12 cascade. 


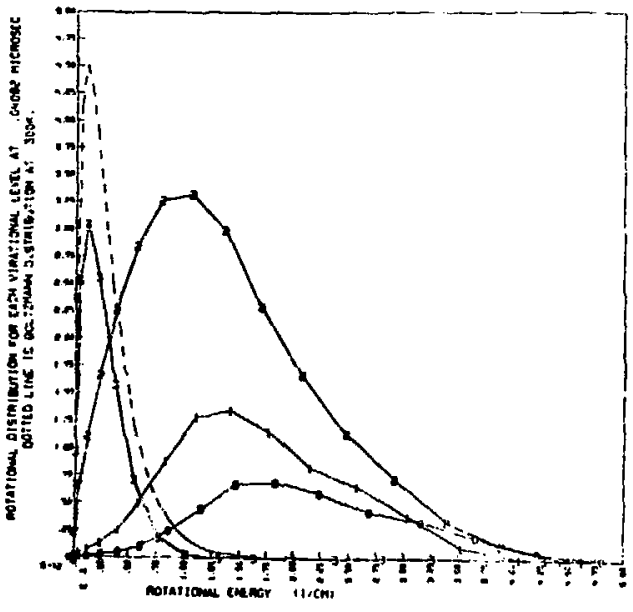

Fig. 8.21 Population distribution before relaxation becomes dominant.

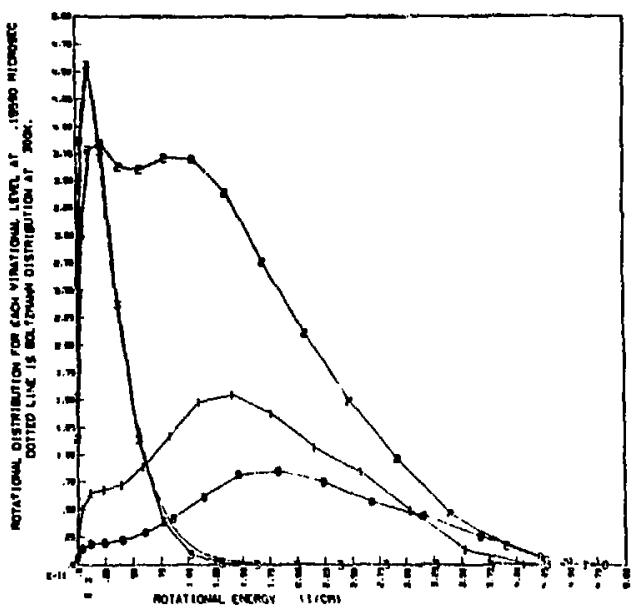

Fig. 8.22 Population distribution at 155 nanoseconds showing a double hump.

용 


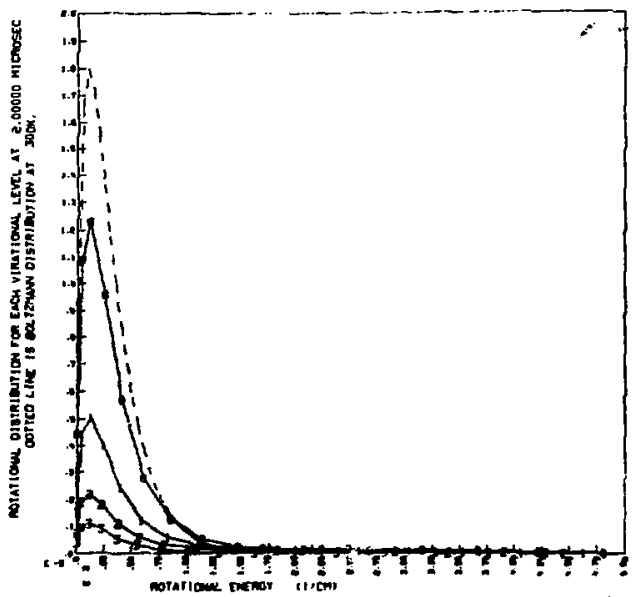

Fig. 8.23 Population distribution at 2.0 microseconas. 


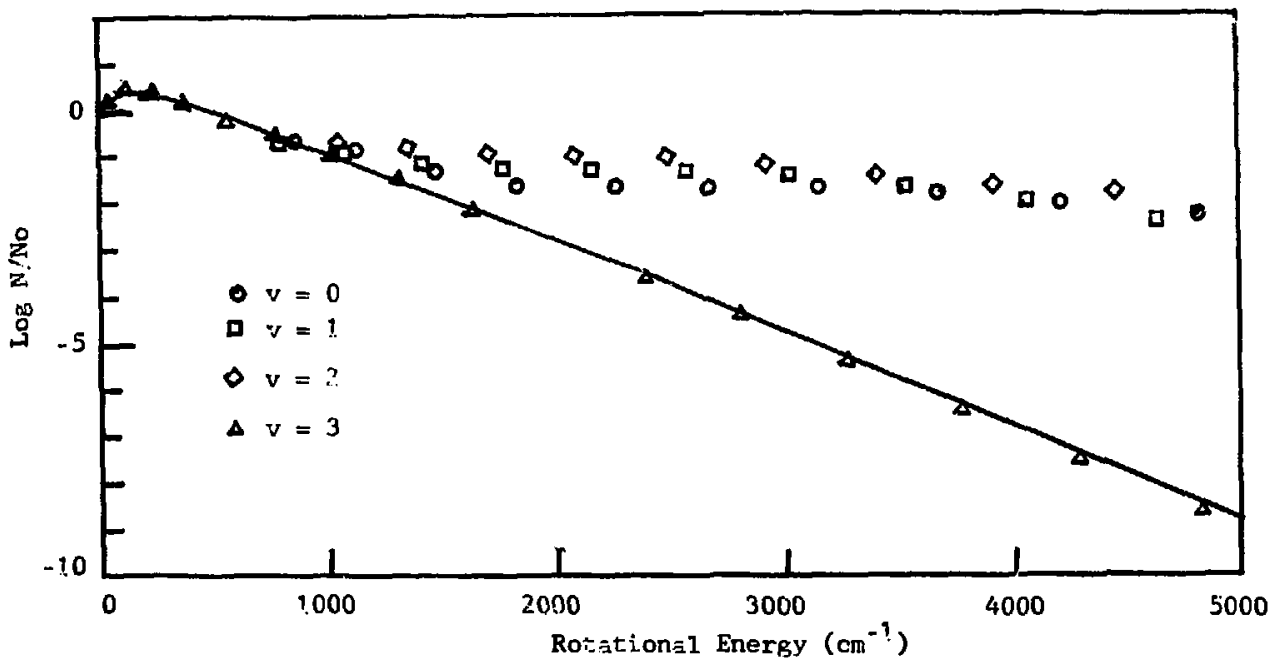

Fig. 8.24 Semi-logarithmic plot of population distributions after 2.0 microseconds. The solid curve is a Boltzmann distribution at $300 \mathrm{k}$.

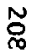




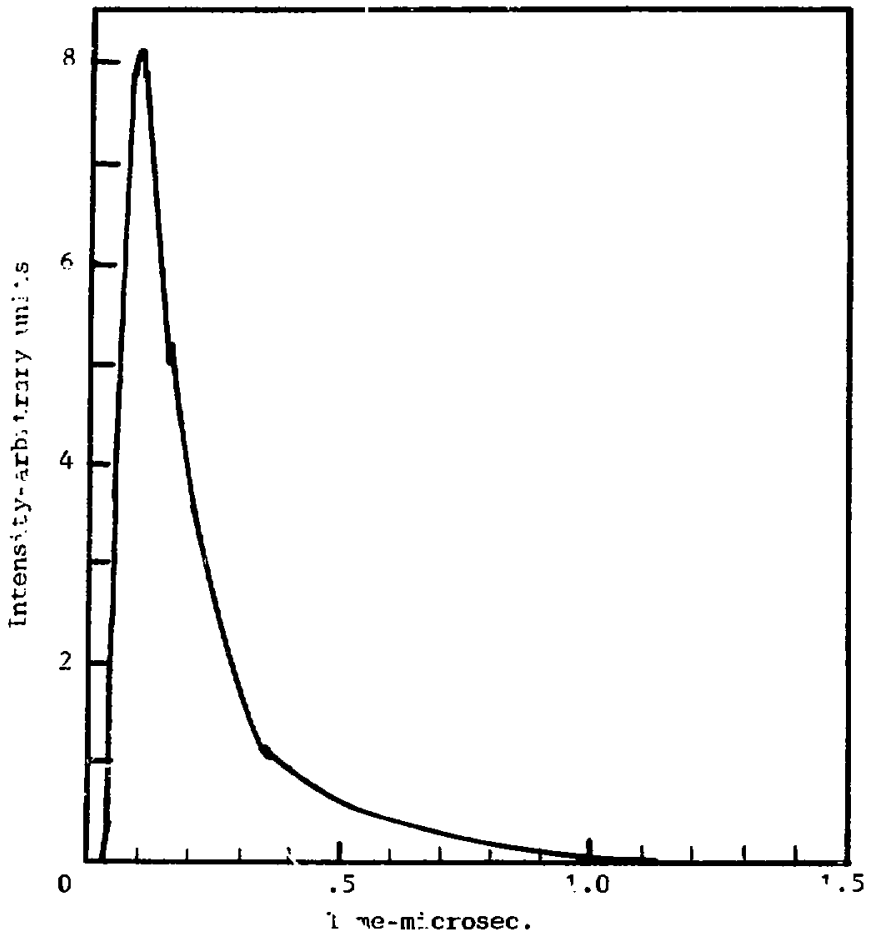

Fig. 8.25 Tot 11 1.iser power is simulited by the RESALE computer code ícourtesy of L. Ripagnani, (973). 
9. VARIATION OF: PARAMETERS

We have now established the validity of the munerical molel by comparison with experiment. he have also used the model to obtain insight into details of the operation of the laser. We will now use the model to study the efrects of varying a number of parameters .

The first parameter to be studied is the rotational relaxation rate. Related to this is the addition of diluent., the variation of the rotational dependence of the rotational relaxation rate, and simulating earlier codes by using a very high rotational rate. The effect of varying the vibrational rate and the fraction of $\mathrm{Nl}_{3}$ which is dissociated will be studied. Finally we will examine the effects of varying the temperature and the total pressure of the $\mathrm{NF}_{3}+\mathrm{H}_{2}$ mixture.

\subsection{Variation of Rotational Relaxation Rate}

Because there was very little experimental data avai lable on the rotational zelaxation rate, the first parameter to be varied was the rotational relaxation rate. An upper limit on the rotational relexation rate is furnished by the experimental values of $\mathrm{HF}$ self-broadening. This is a soft upper limit in the sense that other collision partners may be more or less efficient than $\mathrm{HF}$ in causing line broadening. The code assumed that all molecules were equally effective at line broadening and used the experimental rate 
for HF self-broadening, which is roughly ten times gas kinetic collision frequency. For the nominal case (Fig. 8.1) the rotational relaxation rate was set at $1 / 2$ the linewidth, i.e. 5 limes gas kinetic.

Figures 9.1 to 9.4 show wavef orms for a sequence of increasing rotational relaxation rates with other variables held constant. In Fig. 9.1 the rotational relaxation rate is only one-half or gas kinetjc and the output is largely a number of spikes. This is similar to the output of the unstable laser (Fig. 6.4a). In Fig. 9.1 the line broadening rate is half the nominal value, namely, $5 x$ gas kinetic. The spiking is due to the low relaxation and line broadening rates.

Figure 9.2 shows the waveforms with the rotational relaxation rate set at $2 x$ gas kinetic. In this figure, and the following ones, the linewidth is set at the nominal value, $10 \mathrm{x}$ gas kinetic. Although there are still many spikes, the waveforms of Fig. 9.2 are much smoother than those of Fig. 9.1, indicating greater damping of the relaxation oscillations. The damping is partly due to the increased linewidth and partly due co an increased effective pumping rate. The chemical pumping rate into a siugle state does not appear to be large enough to insure stability, but thr higher effective pumping rate due to rotational relaxation iron adjacent states leads to stability. Comparing these waveforms to the experimental (Fig. 3.2) we see that the duration of the calcul: ed waveforms is too long. If we examine the cascades in Fig. 9.2 
we see that all of the wavelorms are similar, and we do nol see the discontinuities caused by J-shifting as in the nominal case. Cascades appear to dominate over rotational rolaxation at this relatively low rotational relaxation rate. The total energy has increased from $1.4 \mathrm{~mJ} / \mathrm{cm}^{2}$ in $F i g .9 .1$ to $1.7 \mathrm{~mJ} / \mathrm{cm}^{2}$ in Fig. 9.2. Figure 9.3 shows the waveforms for a rotationa 1 relaxation rate of $5 x$ gas kinetic. This is identical to the nominal case except that the dissociation is $1 \%$ rather than $1.25 \%$. Except for a reduced height the waveforms are nearly identical to the nominal case. (A calculation was done with the rotational rate $4 \mathrm{x}$ gas kinetic. The only significant difference from the $5 x$ case was about 100 nsec. delay in J-shifting.) The nominal case has already been discussed in considerable detail, so no further comrent is necessary here, except to note that the total energy has increased to $1.9 \mathrm{~mJ} / \mathrm{cm}^{2}$.

Figure 9.4 shows waveforms when the rotational relaxation is set equal to the linewidth, i.e. $10 x$ gas kinetic. The duration of most of the pulses is shorter and the energy is slightly greater. In some respects the $10 \mathrm{x}$ gas kinetic rotational case agrees better with experiment than the nominal case. The V3-2 and V2-1 transitions turn on at about the same time, the V2-1 P5 is more nearly the right width and the V2-1 P3 is a single spike. In other respects the agreement is worse. The P3 and P4 pulsewidths are too narrow and the interval between turn-on of the V2-1 and V1-0 transitions is too shor:. In most other respects the $5 \mathrm{x}$ and $10 \mathrm{x}$ waveforms are similar. 
The case with rotational rate $5 x$ gas kinetic was chosen as nominal partly because it was felt that there should be significantly more phase-changing collisions than collisions which actually changed the quantum number. This may not be a correct assumption.

The total energy is seen to increase with increasing rotational relaxation rate. This is plotted in Fig. 9.5. This effect is exaggerated partly due to terminating the calculation at 2 microseconds, cutting off 20 to $30 \%$ of the total energy. When the calculation was extended to 5 microseconds (at $1.25 \%$ dissociation) the energies were 2.92 and $3.04 \mathrm{~mJ} / \mathrm{cm}^{2}$ at 2 and 5 times gas kinetic. Two mechanisms can contribute to the increased energy. First, the populations on the stronger transitions are five to ten times the threshold inversion, and are nearer threshold on the weaker transitions, As a result, a small but significant fraction of the population is used in maintaining threshold inversions (in addition to the fraction of the populations in the ground state). With higher rotational relaxation rates fewer transitions lase so fewer threshold inversions must be maintained. Second, and perhaps more important, at lower rotacional relaxation rates the higher $J$ states have larecr populations. These transitions may lase for a sthort time then be unable to sustain threshold inversion and turn of $f$. In that case a large fraction of the population is found in states which don't contribule to the lasing. As the rotational relaxation rate is increased this fraction of the 
popularion finds its way to the lasimp transitioms and adds to the power out put.

\subsection{Addition of biluent}

Experimentally it was foum that adding dile: at on

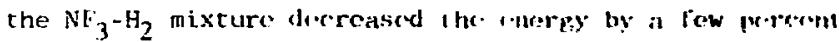
when the diluent was about $50 \%$ ol tho rotal mix. Howider. when the diluent was helium or No lhe laser chnrry increasod under rertain condirions (Poirson, (2ta1,. 1973a). Thr experimental results with helium als a diuent atr shown in Fig. 9.6. The increase may oceur becalse lhe di luent alleres the elficicncy of the discharge in yieldiug, al axns; or it may occur because the diluent keeps the remperature. lowitr. The fact that increasing rotational relaxation rates pave larger energy supgested that the increased encrey with idded rliluent mipht be dur to an increaseyl rotational relaxation rate.

To test this, calculations were performed simulating: the addition of helium. Adding holitm increased the rotationa? and vibrational relaxation rates plus the linewidth. The results are shown as solid circles and triangles in Fig. 9.5 for helium partial pressures of 5,15 and 45 Torr, with the $\mathrm{NF}_{3}+\mathrm{H}_{2}$ total pressure held constant at 15 Torr. No rise j. $n$ output energy with jncreased helium was found; instead the nnergy was found to drop a few percent. The drop is due to the increased throshold inversion resulting from the increased 
lthowidch. The increased Bibrational rate was not sufilcient 10 indse the oneray drofo since hellum is many orders of

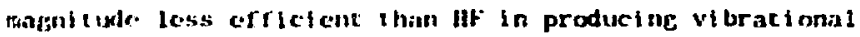
reliaxilion.

The ralculated ristits are in aerectactit with experiments using: Ar, $\mathrm{N}_{2}$, and $\mathrm{kr}$, hue not with experiments usiftr:

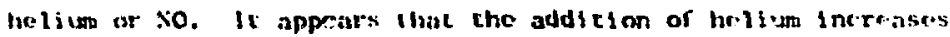

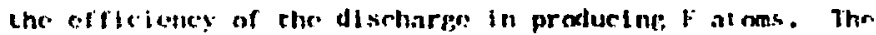
temp:raturn chinger should not produce the incrense because

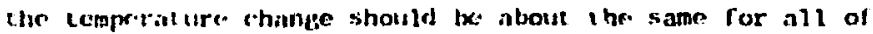
those di luevhs.

Tige calculated wavelorms with added heltati are show In Flf,s. 9.7 and 9.8. Thorr wero no experimental waveforms to compare with the calculated waverorms.

Two trends are cleir in the calculated waveforms. The wiveforms nre smoother, and with hipher diluent the spikes are hifher and the pulses narrower. (Note that the power seale. is twice as large in fif. 9.8.) The hipher splkes are due inatnly to the increased rotational rate. The narrower pulsewidths correlate with higher powers to keep the rotal energ: about the same. Laser powcrs are limited by the pumping rate and incruased rotational relaxation rates mean laster pumping from the ontifer vibrational lavel into a single stare. The smoother mulses ire: a result of chr increased linrwidth and inireased pumpink rale, heciust both damp the relaxation ostrillations . 
The dillent also weake'sls or prevents oscillat $i$ (u) on $P 3$ and $P 7$. The increased linwidth rilises the Itreshold inversion, and the increased rotitional rolaxition depletess these populations. The conbined result coneentrates the lasing: in $P / 4$ throuph $P G$. In peneral, higher rotalional yelaxat ion ratces leghl 10 concent rate the lasing, in a few transitions.

\section{3 Calculation to Approximate Rotational Equi 1ibrium} Earlier mudels of the HF rhonical laser have issuned rot atj mal cyujlibriun within each vibrational level. This is "quivalent to an inlinite rotational relaxalion rate, except that reprorimental linewidths are used to ralculate the gain. (Pty sicall). the collision broadened linewidth is proportional to tho rutational relaxation rate because any collision rasing rolational relaxation will destroy the phase coherence it that molceule.) To approximate the inlinite rotational relaxation rate a calculation was done with the rotational re:Laxation rate 200 times gas kinetic, or 40 times the nominal baluc. The linewidih was left at its nominal value co simulate carlier nodels. A larger rolational rate would have heen desirable, but computation time is roughiy proportional to the rotational relaxation relaxation rate, so the factor, 200 times gas kinetic, was chosen ro keep the computation time reasonaule.

Models which assume rotational equilibrium allow only one transition at a time to lase between any two vibrational levels. The transition with the highest gain lases and others lack suflicient gain to lasc. As populations 
charge the lasing can switch instantameous $1 y$ to another transition. The calculated waveforms, Fig. 9.9, show approximately this behavior. There is some overlap in waveforms but there is a distinct tendency for only one transirion at a time to lase between each pair of vibrational levels. In addition, cascade behavior seems to be totally missing, and lasing at high $\mathrm{J}$ was suppressed. The total energy is greater than earlier case because of the increased rotational relaxation rate. The total energy is $2.67 \mathrm{~mJ} / \mathrm{cm}^{2}$ in 2 microseconds as conpared to the nominal value of $2.40 \mathrm{~mJ} / \mathrm{am}^{2}$ at 5 times the gas kinetic collision frequency.

This calculation shows that the IXION code, in the limit of infinite rotational relaxation rate, will give the same results as earlier models, except for differences due to using semi-classical laser equations rather than a steadystate approximation.

\subsection{Modification of the Rotational Relaxation Rate} as a Function of Rotational State

The variation of the rotational rate, as used in the IXION code, was obtained by extrapolating from relared experimental data, enlightened by a simple theory. The experimental ditha consisted of Polanyi and Woodall's work (1972a) on rotational relaxation of $\mathrm{HC}]$, and $\mathrm{HF}$ self-broadening data (Mercelith, 1972) which gives an upper limit on the rotational relaxation rate. A calculation was performed to rest the sensitivity of the model to the assumptions. 


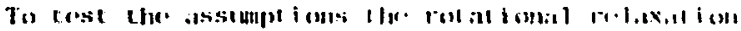

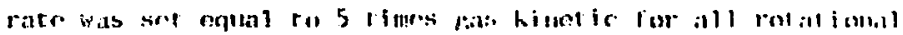

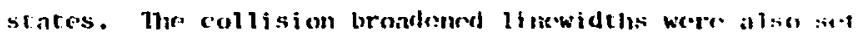

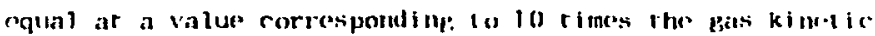

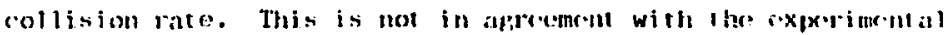

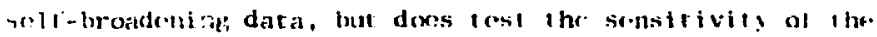
norle 1.

The calculated wavetorms, shown in fik. 9.10, differ significautly from the nominal rase of Fis. 8.1. The PG and ligher wavolorms are entirely missing in $i \mathrm{E} .9 .10$, as is the V3-2 p5 waveform. This is partly due to the hithor rotational relaxation rate ar. high $J$, and partly due to the higher threshold inversion at high. I caused by the increastod 1inewidth. Examination of the rime history of the inversions shows that thnse transitions which did not lase did not reach threshold. Some of them would have reached threshold i1 the populations had not been clamped by Lasing, on the P3 through P5 transitions. Also they might have reached threshold if the threshold inversion had decreased witl: increasing $J$ as in the nominal case.

If sone respects $\mathrm{Fig} .9 .10$ compares well with the waveforms at very high rotational rate (F'ig. 9.9). This is not surprising because setting all rotational rates equal to the maximum greatly increases the eflective rotational relaxation rate. The differencos between the two sets of waveforms arise mainly from the diflerent threshold inversions. The similarities arise out of the increased rotational relaxation rate. 
The rotal energy with equal rotational relaxation rates is $2.56 \mathrm{~mJ} / \mathrm{rm}^{2}$, a lew percent higher than in the nominal cast. (Hig. 8.1) which had a total energy of $2.40 \mathrm{~m}$.J at 2 microseconds. This is consistent with the increased ef fective rotatj onal relaxation rate.

The waveforms bastod on the nominal model agree better with experiment than do those of Fig.9.10. This indicaces that the nominal case is a better descripcion of experimental reajity. Unfortunately, the high $J$ waveforms were not measur'l ixperimentally. The sensitivity wasn't high enough to show that high-J transitions didn't lase weakly, as calculated. Orher experiments (Pummer and Kompa, 1972) show that high I transitions do lase under some conditions Experiments could be performed under conditions which were more sensilive to the assumptions in the model regarding the $j$-dependence of the rotational relaxation rate.

\subsection{Vibrational Relaxation Rate}

The anomalously high vibrational relaxation rate of $\mathrm{HF}$ has frequently been blamed for the low olitput of HF chemically pumped lasers. For the pulsed laser considered here, vibrational relaxation was expected to be unimportant. compared to depletion of the flourine acoms. This would not be true of a laser based on a chain reaction, and might not be true of a laser where the dissociation was fairly slow. 
To verily that the vibational relaxationt lat wat w unimportant in thr prosent case a ilumber of ruas witre made al molliples of the mominal vilorational relaxat ion rille. Mu.

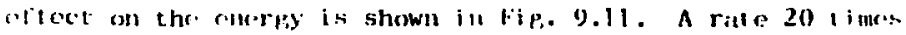
the nominal sitc rostlited in only a $20 \%$ drop in energy. Our "alculation was proformed with all rates nominal except that tac: Vibrational rolaxation rate for $\mathrm{H}_{2}$ was 100 rimes nominal. This results in approximaledy the sane overall relaxation vate as tmiltiplying the nominal tate by 9 . This calculation is rer sellied by an $x$ in fig. 9.11, and falls vary close to the line comnecting other values. In addition, some calculations were performed wh the vibrational relaxation rates for $\mathrm{H}_{2}$ and $\mathrm{NF}_{3}$ erroneously set equal to the rate for $\mathrm{HF}$. This is roughly equivalent to a multiplier of 110 . A dissociation of $5 \%$ was required to obtain a total pulse energy of about $2 \mathrm{~mJ} / \mathrm{cm}^{2}$. This is a reasonable extrapolation from rig. 9.11 .

The waverornts for the case with vibrational relaxation 20 times the nominal rate are shown in Fig. 9.12. The only major difference between those and the waveforms for the nominal case (Fí. B.1) is a slightly reduced amplitude and a slightly faster decay in iritensity.

The calculations presented here show that vibrational relaxation is not an important factor for the condicions studied. It seems unreasonable to think that the vibrational relaxation rate is more than 20 times the nominal value, 
since the nominal value corresponds to vibrational relaxation every 50 collisions with $\mathrm{HF}$. The nominal rate for $\mathrm{H}_{2}$ may be low, but: probably not more than the factor of 100 used in the calculation. The output is even less sensitive to the $\mathrm{NF}_{3}$ rate, because it is so small.

In summary, the calculations were not very sensitive to vibrational relaxation rates within a physically reasonalle rarge. Under other conditions, e.g. chain reaction, the laser could be quite sensitive to the vibrational relaxation rate.

\subsection{Fraction of $\mathrm{NF}_{3}$ Dissociated}

At various times calculations were performed with the number of $\mathrm{F}$ atoms ranging from $1 \%$ to $10 \%$ of the $\mathrm{NF}_{3}$ present. The total output energy was a linear function of the $\mathrm{f}$ atom concentration over this range. This is to be expected from the simple analytical models. The calculation used simplified inodel of the chemistry which neglected consumption of hydrogen and recombination of $F$ atoms. Analysis of the rates shows that $F$ atom recombination should not be significant under the conditions which were $c$ msidered. Consumption of $\mathrm{H}_{2}$ would introduce some non-linearity for higher percentapes of $\mathrm{F}$ atom, but should not be significant at the nominal value, $1.25 \%$ dissociation.

\subsection{Variation of Laser Output with Temperature}

Calculations were done at constant temperatures from 200 to $700 \mathrm{~K}$, with the pressure held constant at 15 Torr. 
The total laser enerpy is shown as a runction of temperature. in $F j B .9 .13$. The energies at 200 and $300 \mathrm{~K}$ were based on calculations for a 5 microsecond scale, in order to include the entire pulse energy. At higher temperature the pulse is essentially complete by 2 microseconds, which was the scale ror the calculations. The two time scales should introduce lictle error. The decrease in energy with increasing temperature is mainly due to the decreasing density (molecules $/ \mathrm{cm}^{3}$ ) as we increase the temperature at constant pressure. if the density is held constant as the temperature is increased to $700 \mathrm{~K}$ the pressure is about 36 Torr. A calculation was done at 36 Torr and $700 \mathrm{k}$; the total laser energy for this case is shown as a triangle in Fig. 9.13. The energy at 36 Torr and $700 \mathrm{~K}$ is about $15 \%$ smaller than that at $300 \mathrm{~K}$ due to the $\mathrm{HF}$ being distributed over mcre rotational states.

The waveforms calculated for 15 Iorr at $700 \mathrm{~K}$ are shown in Fig. 9.14. The amplitudes are much smaller than at $300 \mathrm{~K}$ bacause of the reduced density. The pulse widths are comparable because the reduced density is compensated by the increased rate constants. It is fortuitous that the two effects tend to cancel, because the rate constant could have a different temperature dependence.

The waveforms calculat ad for 36 Torr at $700 \mathrm{~K}$ are shown in Fig. 9.15. This case has the same number density as 15 Torr at $300 \mathrm{~K}$. The amplitudes at $700 \mathrm{~K}$ are about double those at $300 \mathrm{~K}$ and the pulsewidths are about a half, so that 
the total energies are nearly the same. This is due to the increased reaction rates. The lasing is shifted cowards higher $J$ at $700 \mathrm{~K}$. This is expected because the population distributions are similarly shifted towards higher $\mathrm{J}$ at $700 \mathrm{~K}$. The principal effect of raising the temperature is to decrease the pulsewidth and increase the amplitude, provided the density is unchanged. The energy is decreased slightly because the molecules are distributed over more states. In addition there is the weakening or disappearance of P3 and P4 transitions and the strengthening of $\mathrm{P} 7$ and $\mathrm{P} 8$ transitions.

\subsection{Total Output Energy as a Function of Total Pressure} In Section 3 we noted that experimentally the total laser energy did not increase lineary with increasing total pressure. In Section 4.4 we discussed possible mechanisms for this, based on an approximate analytic model. We were able to postulate phenomena that would cause non-linear output with pressure, but only at much higher pressures than were used in the experiments.

These mechanisms were not included in the IXION code, so total calculated laser output energy was expected to increase linearly with pressure. Never-the-less, calculations were done at total pressures from 1 to 310 Torr to see if there were any unexpected effects. In addition, there were experimental waveforms at. 60 Torr (Fig. 3.4) to be compared with calculated waveroms. 
The calculations showed, as expected, that total output energy was roughly proportional to pressure. The results are plotted in Fig. 9.16. At low pressures the total energy is less than the linear prodiction because a larger [raction of the molecules is used to maintain a threshold inversion.

The experimental point at 60 Torr is less than half the calculated energy at 62 Torr. This indicates that the non-linear variation of energy with pressure is not due to Iaser dynamics. To simulate reduced dissociation efficiency the calculation was repeated assuming a dissociation of $0.6 \%$. This gave better agreement with experiment as shown in Fig. 9.17. There is reasonably good agreement with experiment, but not as good as at 15 Torr. In particular, pulsewidths seem too long and the calculated intensities on P7 are too low.

In an attempt to get better agreement with experiment thic calculation was repeated with the temperature increased to $400 \mathrm{~K}$. (For constant density the pressure was 77.5 Torr.) The pulsewidth was shortened significantly and the P7 transitions compare better as seen in Fig. 9.18. The calculated P3 transitions were deleted from $\mathrm{Fig} .9 .18$ because they were too weak to be seen at this scale. In spite of the better agreement with experiment the calculated J-shifting seems to occur later than the experimenta].

The fact that the P3 transitions were observed experimentaliy tends to indicate low temperature but the 
short pulse and early J-shifting seem to indicate a high temperature, A reasonable explanation for this would be a rapid1y rising temperature, increasing a few hundred degrees by the end of the pulse. Estimating the appropriate temperature rise requires a detai led knowledge of the discharge physics which is beyond the scope of this work. It also appears that it would be very difficult to measure experimental1y。

The differences between 1 ow and high pressure waveforms are surprisingly small, as seen by comparing Fig. 9.19 (1 Torr) with Fig. 9.20 (310 Torr). The apparent1y narrower pulsewidth at 300 Torr is deceptive, since the scale at 300 Torr is $1 / 100$ of that at 1 Torr. The 300 Torr pulse is actually wider, relative to chemical reaction rates. This is largely due to the fact that the discharge is still creating now $F$ atoms near the end of the pulse. In fact, the sinusoidal shape of the discharge current waveform can be seen near the end of the 310 Torr pulse. Experimental waveforms at these pressures were not available to compare with calculations. 

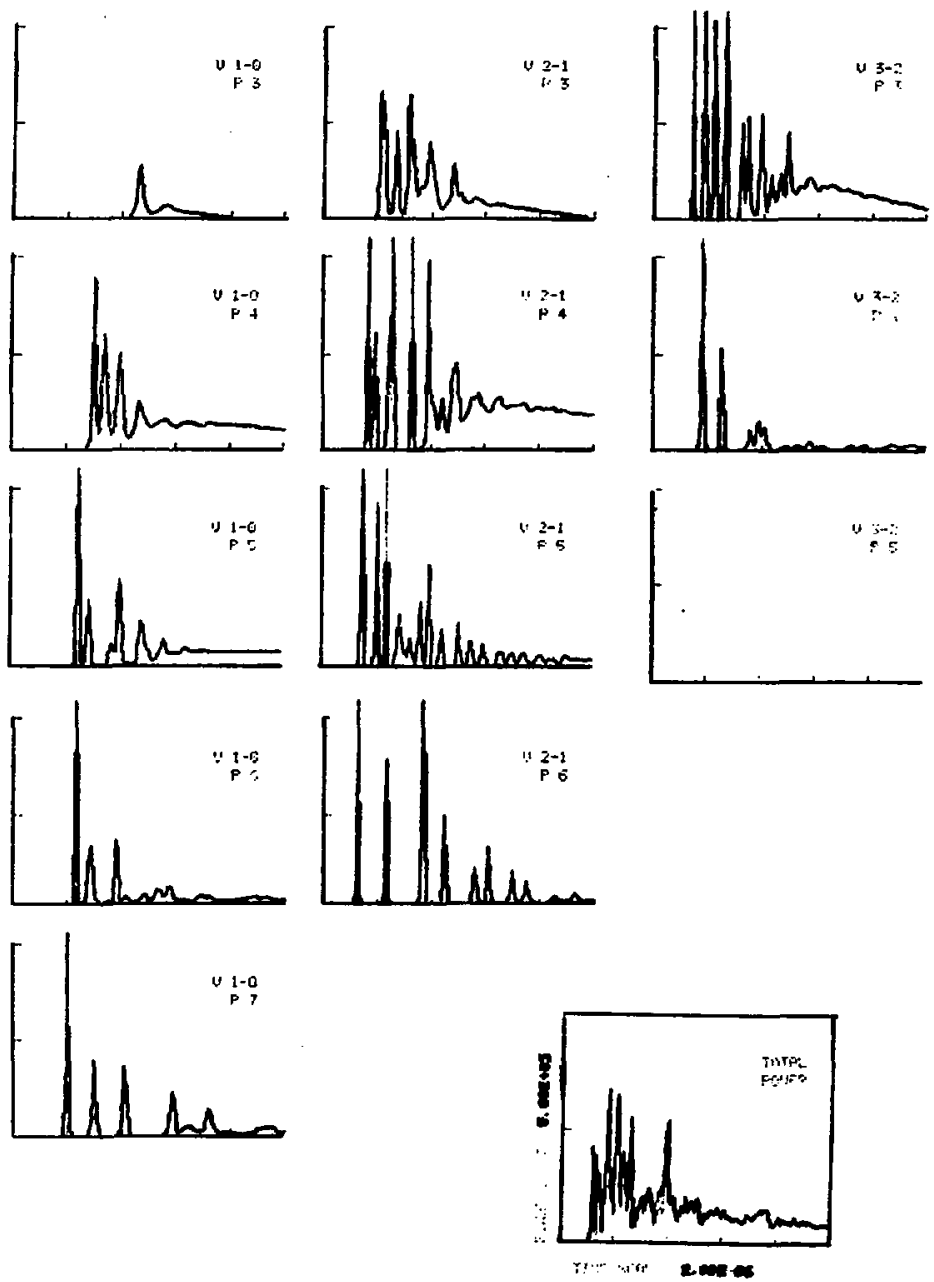

Fig. 9.1 haveforms at 15 Torr when the rotational relaxation rate is $1 / 2$ gas kinetic and linebroadening cross section is $5 \mathrm{X}$ gas kinetic. One-percent of the $\mathrm{NF}_{3}$ is dissociated, total laser energy is $1.42 \mathrm{~mJ} / \mathrm{cm}^{2}$. 

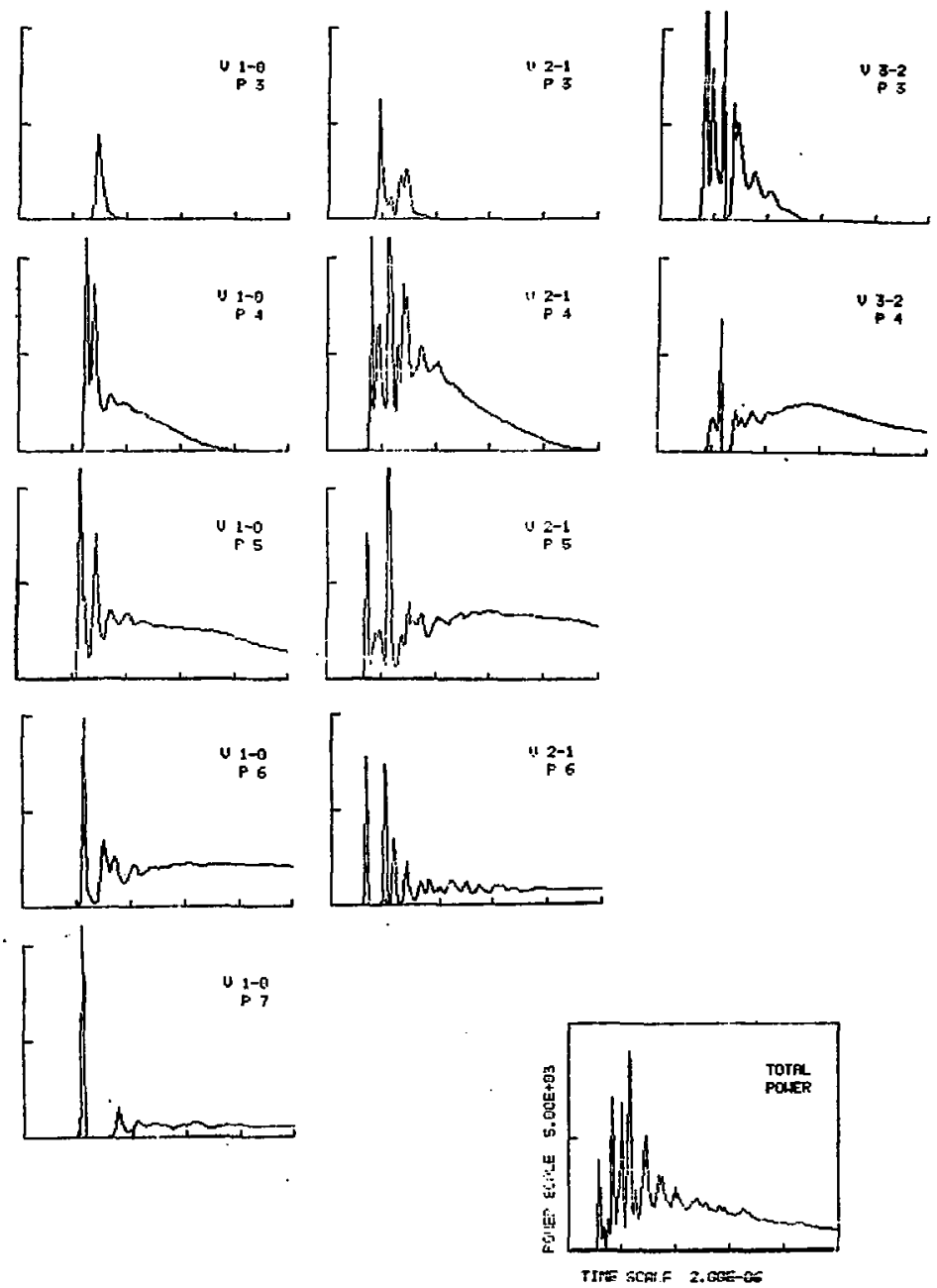

Fig. 9.2 haveforms at 15 Torr when rotational relaxation rate is $2 \mathrm{X}$ gas kinetic and linebroadening is $10 \mathrm{X}$ gas kinetic. Dissociation is $1 \%$, total laser energy is $1.71 \mathrm{~mJ} / \mathrm{cm}^{2}$. 

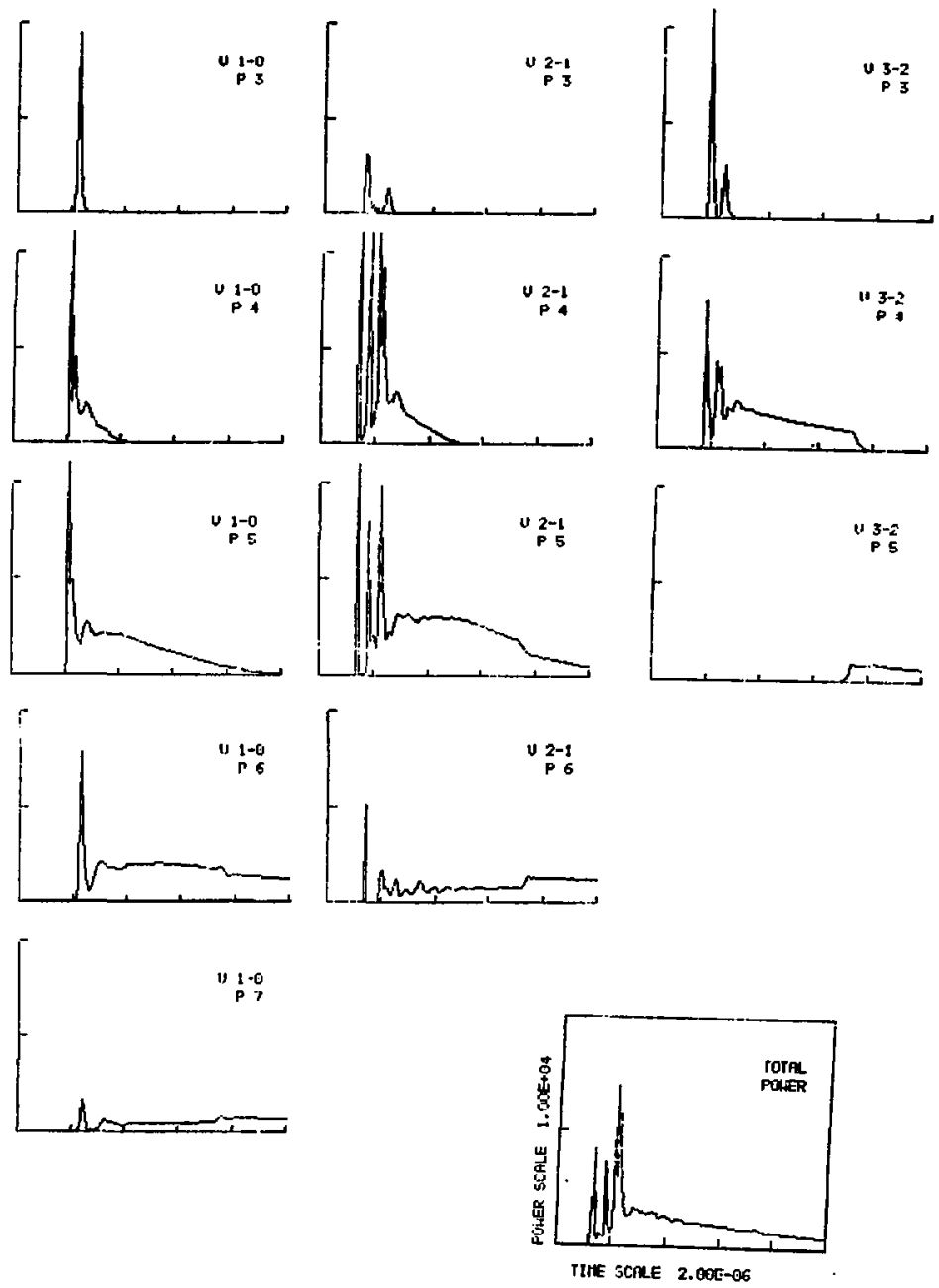

Fig. 9.3 Waveforms at 15 Torr when rotational relaxation rate is $5 \mathrm{X}$ gas kinetic and 1 inebroadening $10 \mathrm{X}$ gas kinetic. Dissociation is $1 \%$, total energy is $1.89 \mathrm{~mJ} / \mathrm{cm}^{2}$. 

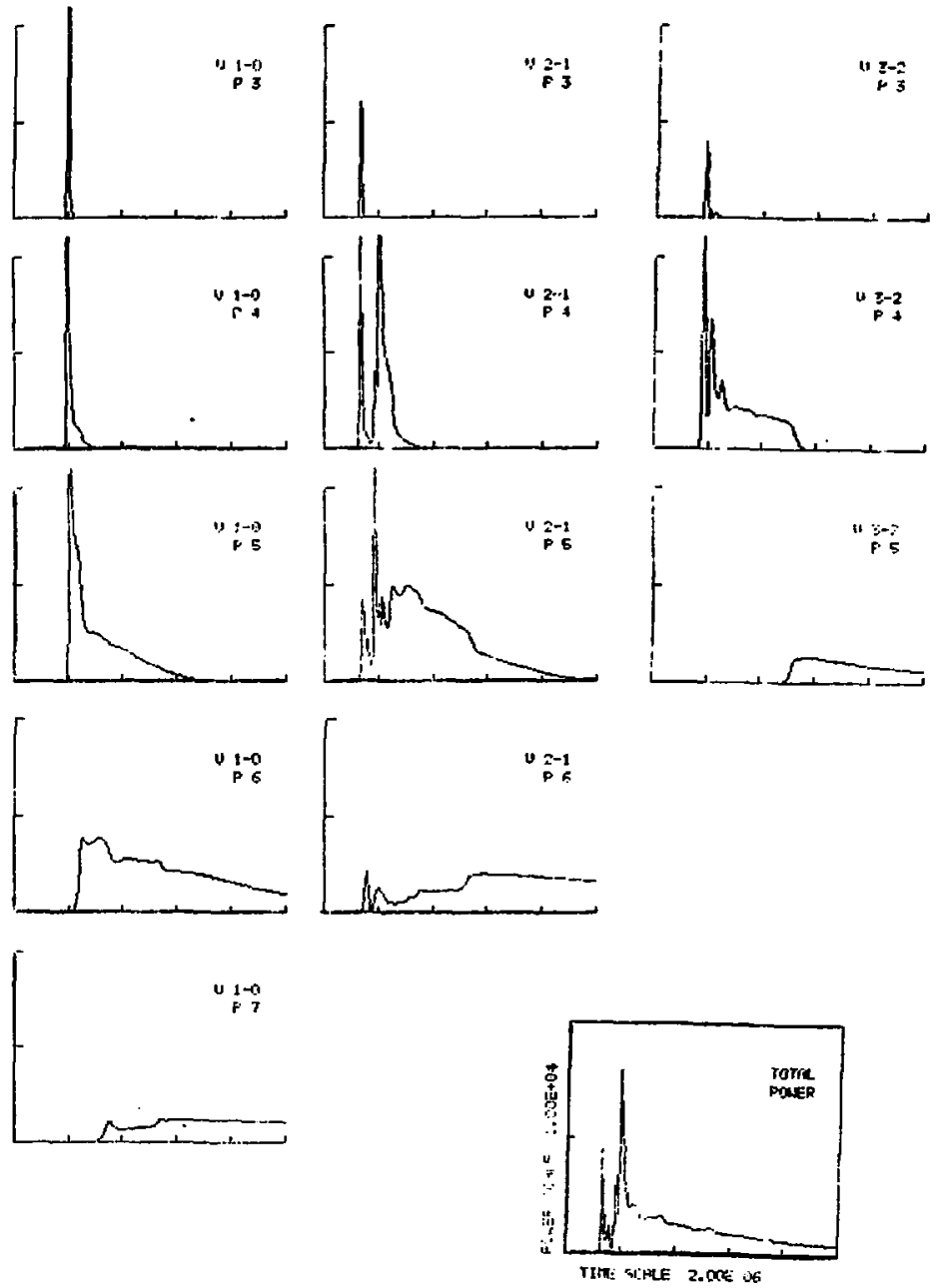

Fig. 9.4 haveforms at 15 Torr when rotational relaxation rate and linebroadening are $10 \mathrm{X}$ gas kinetic. uissociation is $1 \%$, total energy is $1.98 \mathrm{~mJ} / \mathrm{cm}^{2}$. 


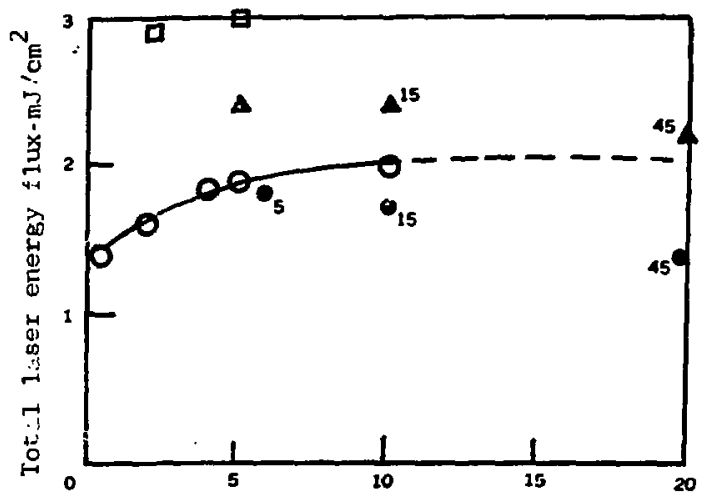

Ro::t_on 11 Relixation Rate'Ghs Kinetic
$\bigcirc\} \%$ dissoc:-ion
$\Delta$ \}?. '5\% dissoc: at:ion
f a msec calculation
a 1.25\% dissocition, $5 \mu$ sec calculation

Fig. 9.5 Varitition of cilculited output ensrgy us. rot:ition il relixition rite normalized to gas kinetic. Numbered points indicate partial pressure (Torr) of helium. 


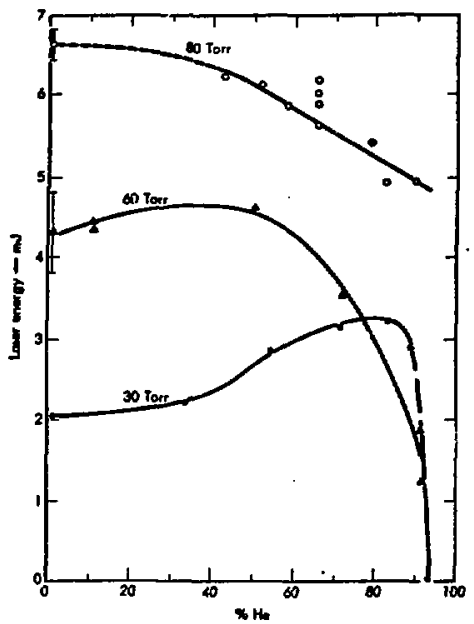

FiE. 9.6 Luser energy versus percent $H_{e}$ for $N F_{2} / H_{2} \geq$ S. discharge time cquals $8 \mathrm{~ns}$, and reactant pressure $\left(P_{\mathrm{N}},+P_{\mathrm{Q}}\right)$ equals (a) 30 worr; (b) 60 torr; and (c) $\mathrm{BO}$ torr.

Reproduced by perrission from far rson, et. a2. (1973e). 

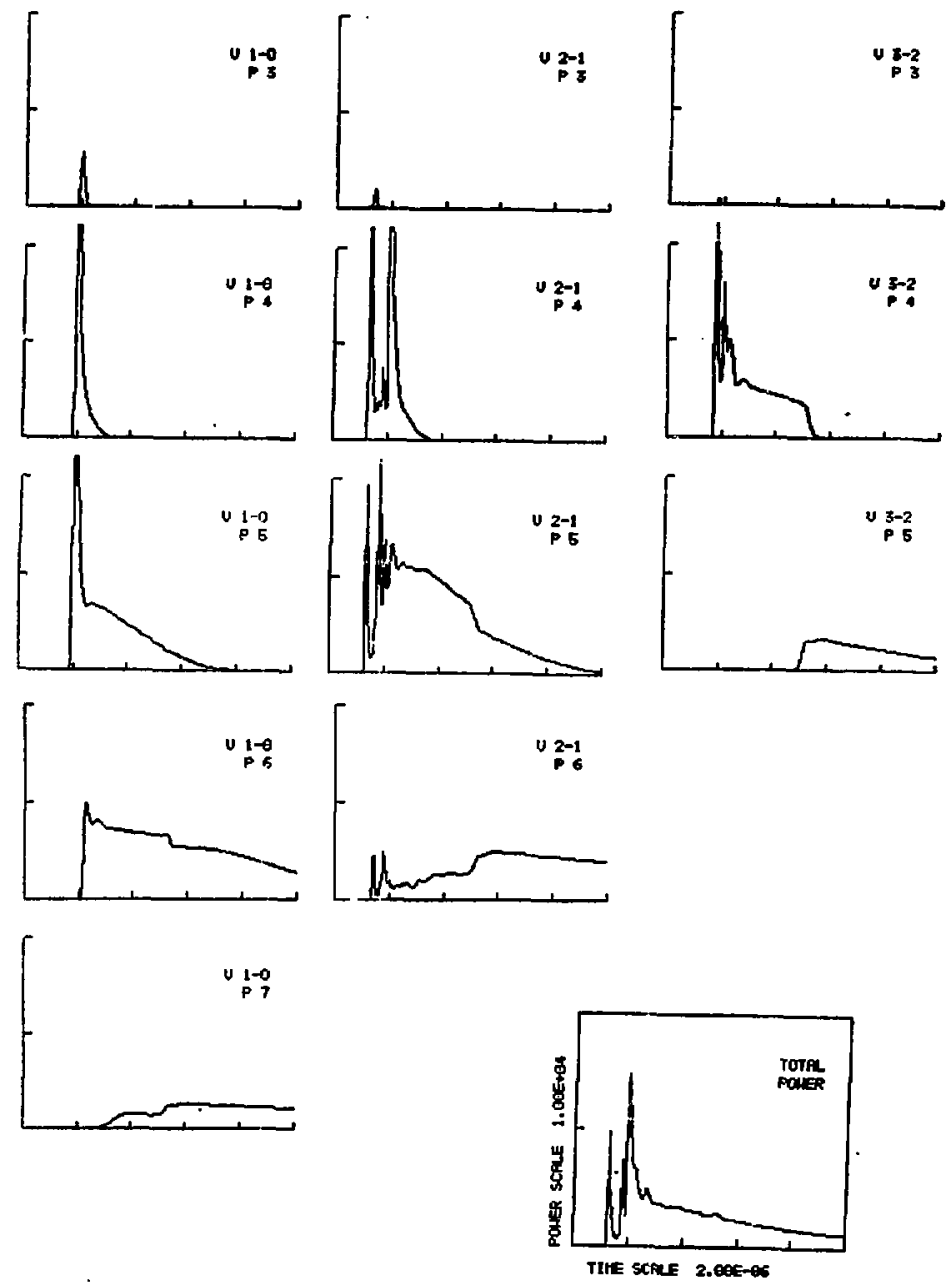

Fig. 9.7 haveforms at 15 Torr when 15 Torr of helium is added, Rotational relaxation rate is $5 \mathrm{X}$ gas kinetic, dissociation is $1.25 \%$, total energy is $2.40 \mathrm{~mJ} / \mathrm{cm}^{2}$, the same as the case without helium. 

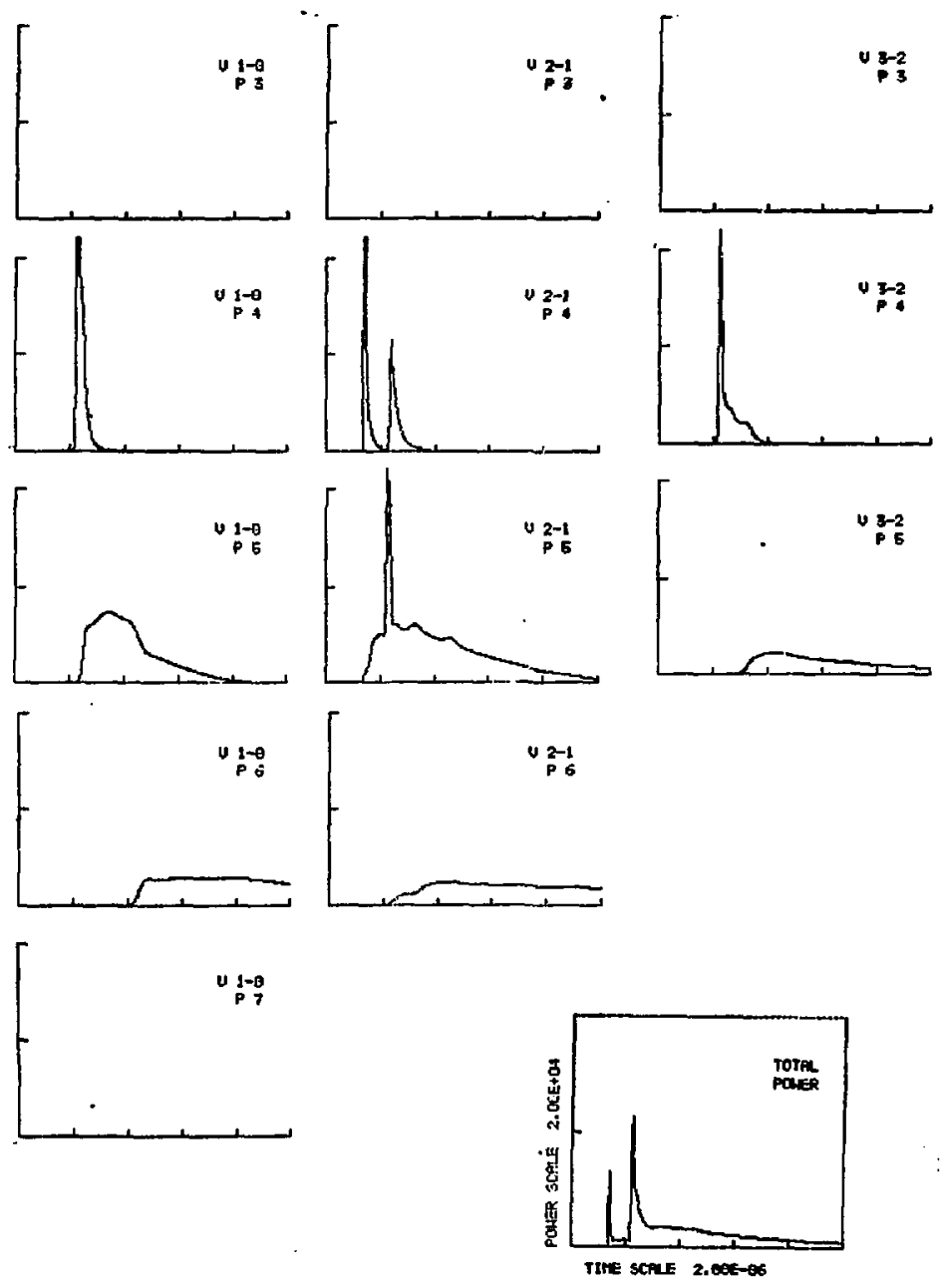

Fig. 9.8 Waveforms at 15 Torr with 45 Torr of helium added. Total output energy, $2.23 \mathrm{~mJ} / \mathrm{cm}^{2}$, is somewhat less than the nominal case. 

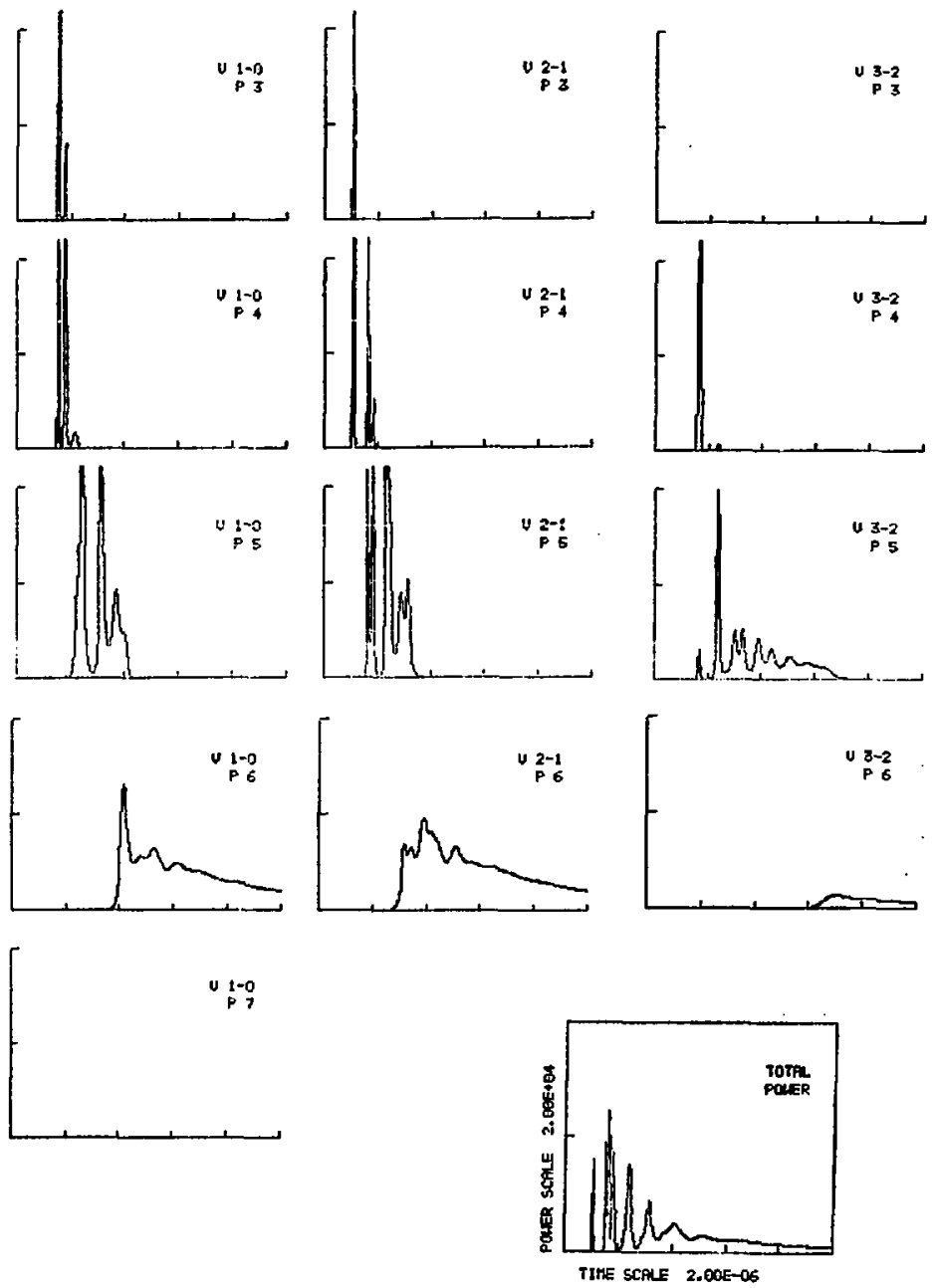

Fig. 9.9 Waveforms at 15 Torr when rotational relaxation rate is $200 x$ gas kinetic to approximate the assumption of rotational equilibrium; Dissociation is $1.25 \%$, total energy is $2.67 \mathrm{~mJ} / \mathrm{cm}^{2}$. 

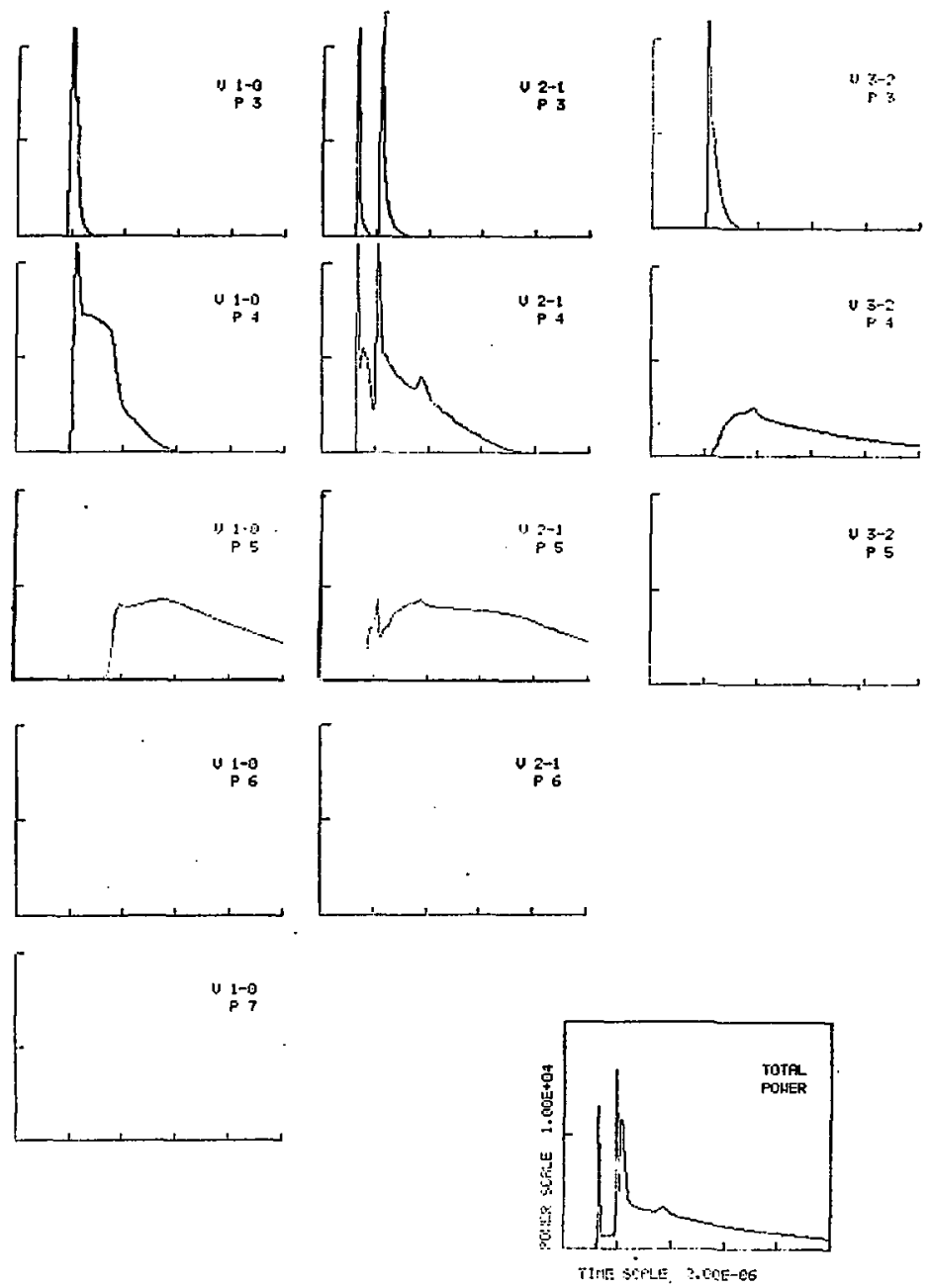

Fig. 9.10 Waveforms at 15 Torr assuming rotational relaxation rate is $5 \mathrm{X}$ gas kinetic for al1 rotational states, independent of $\mathrm{J}$, Dissociation is $1.25 \%$, total energy is $2.56 \mathrm{~mJ} / \mathrm{cm}^{2}$. 


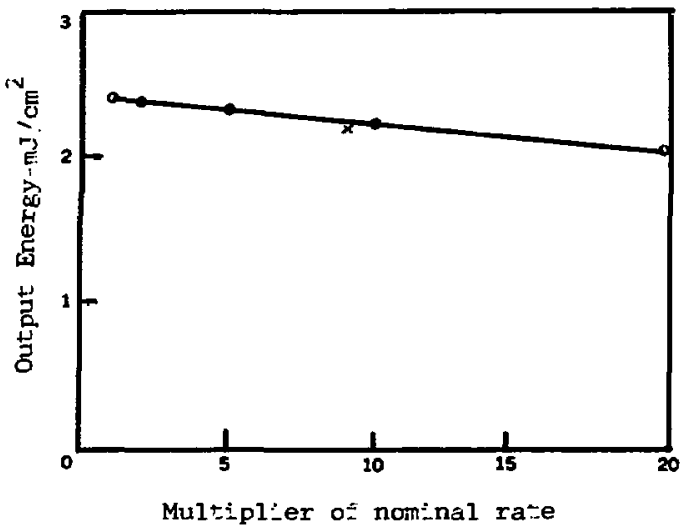

Fỉg. 9.1. Variation of laser output energy as a function of the vibrational relaxation rate. The point (x) represents the case where the vibrational rate for $\mathrm{H}_{2}$ wss 900 times nominal, but other rates were nomin $=1$. The equivalent multiplier is about 9 . 

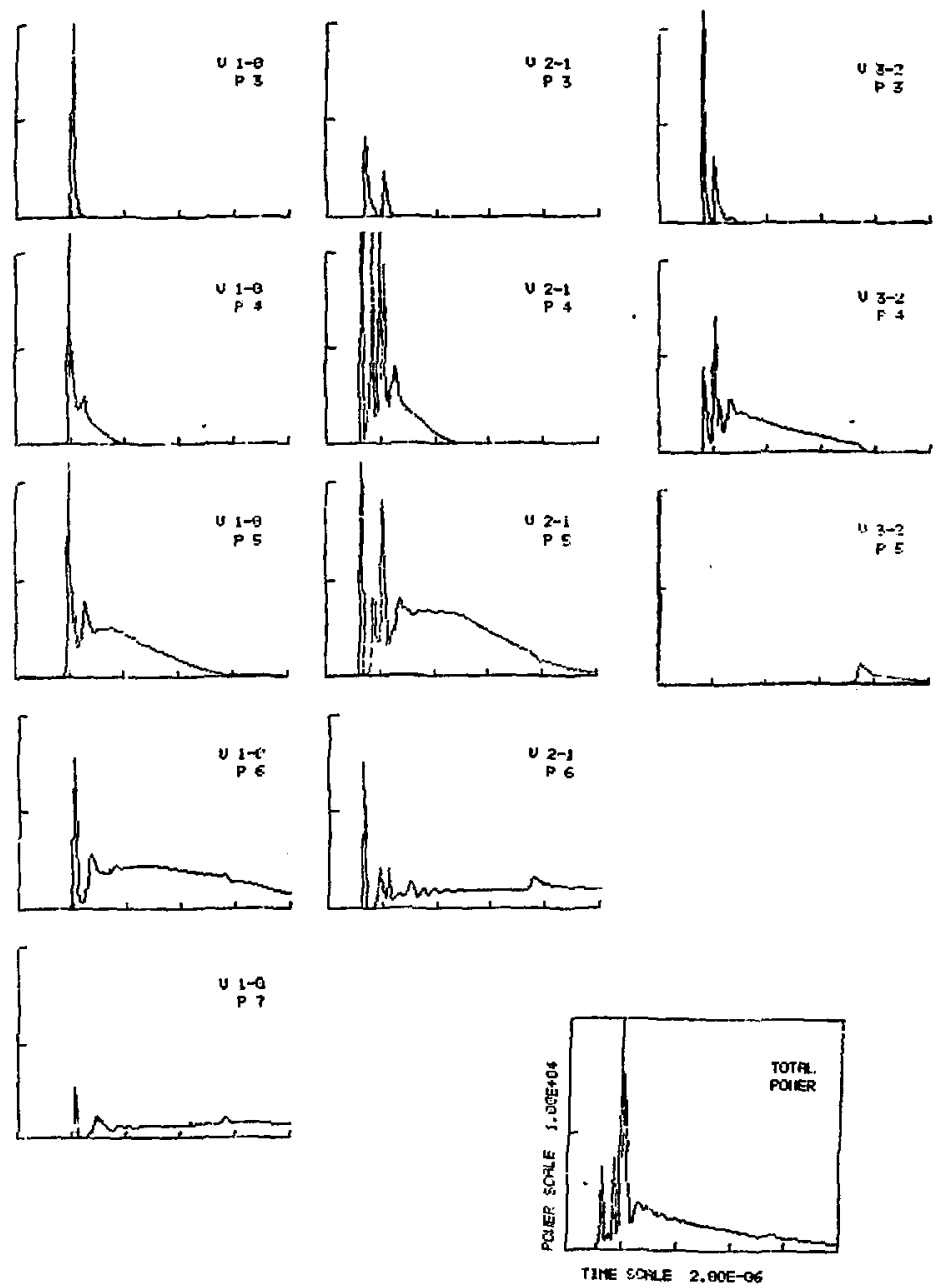

Fig. 9.12 Waveforms at 15 Torr when vibrational relaxation rate is $20 \mathrm{X}$ the nominal value. Other parameters have nominal values as in Fìg. 8.?. Total energy is $2.0 \mathrm{~mJ} / \mathrm{cm}^{2}$. 


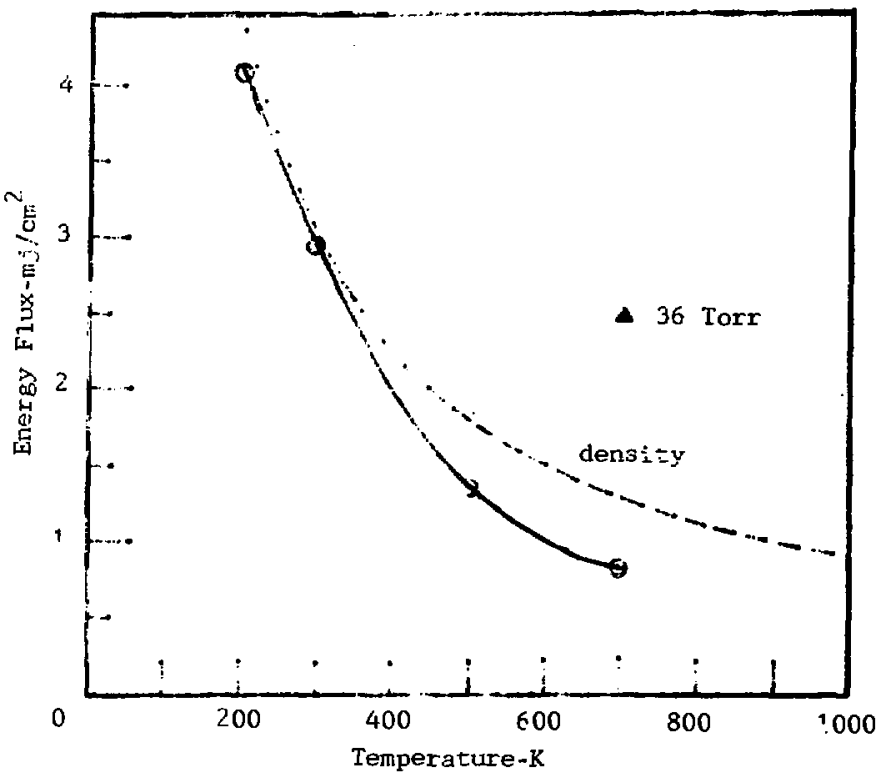

F.g. 9.13 Total laser output energy at 15 Torr as a function of tempereture. The dashed line is the proportional to the density of an ideal gas at 15 Torr pressure. The point marked 36 Torr at $700 \mathrm{~K}$ has the same density as the point with $: 5$ Torr at $300 \mathrm{~K}$. 


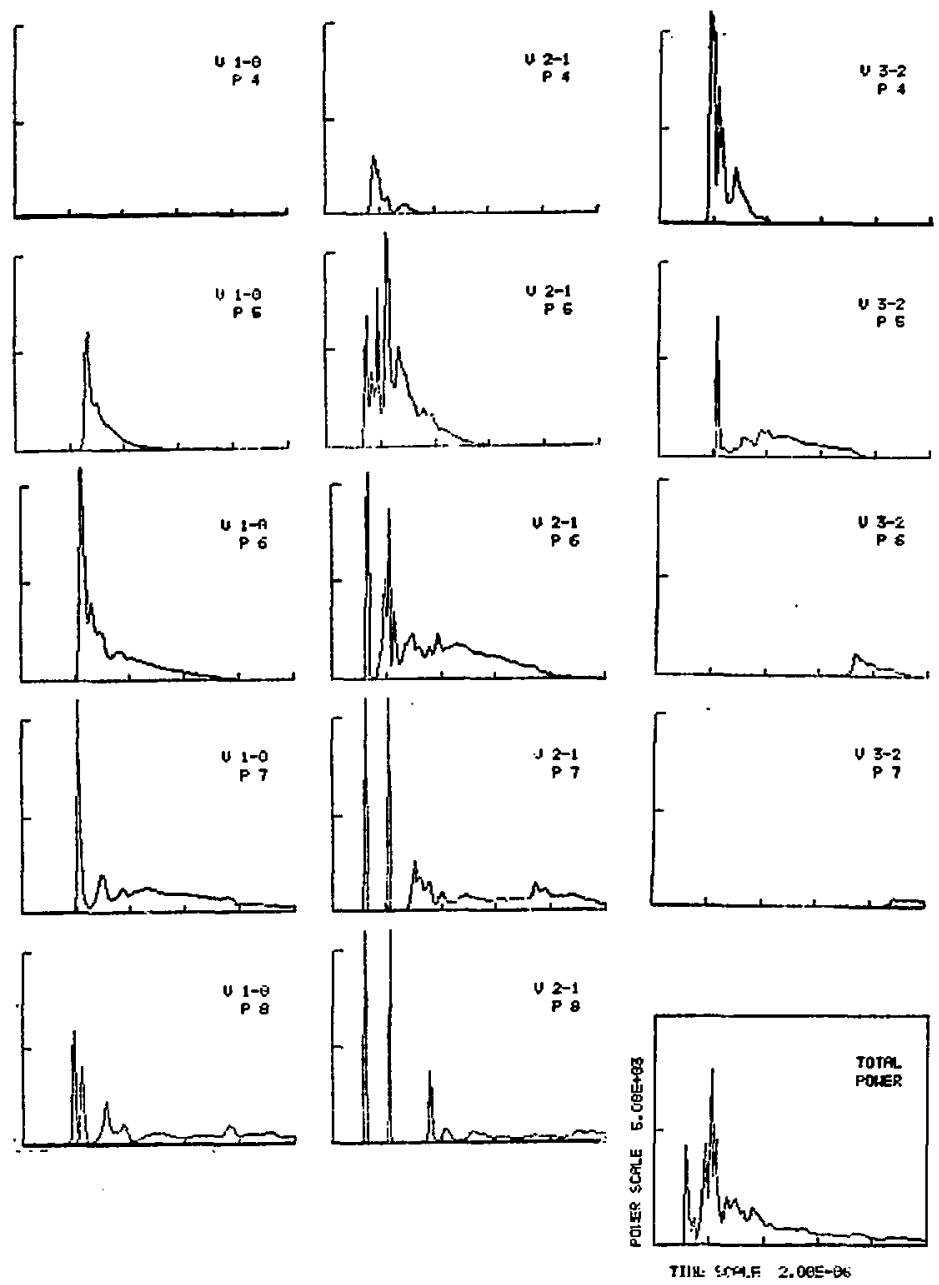

Fig. 9.14 Waveforms at 15 Torr when temperature is $700 \mathrm{~K}$. Other parameters have nominal values. Total energy is reduced to $0.84 \mathrm{~mJ} / \mathrm{cm}^{2}$, mainly because of reduced density. 


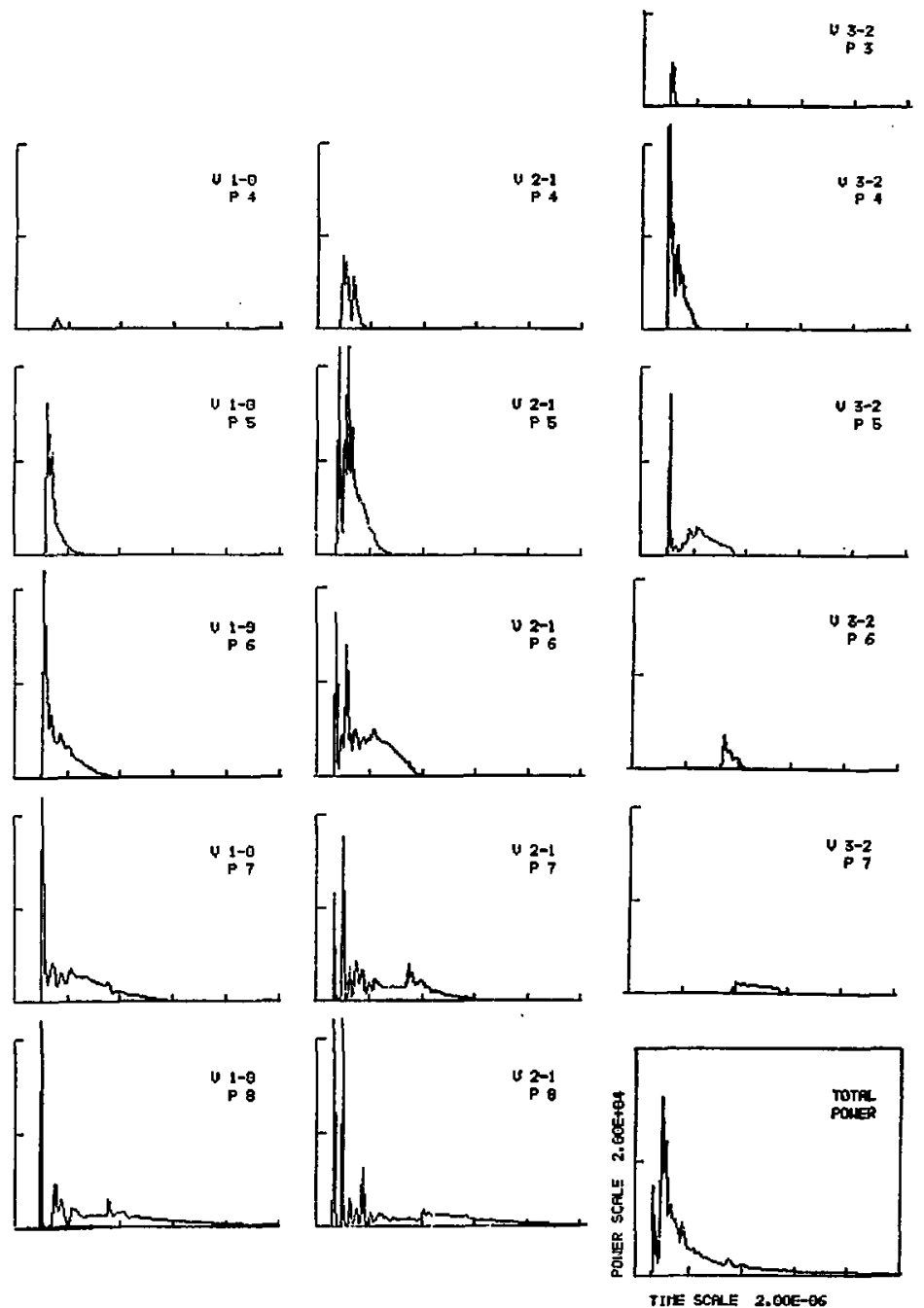

Fig. 9.15 Waveforms at 36 Torr and $700 \mathrm{~K}$,' which has the same density as 15 Torr at $300 \mathrm{~K}$. Energy is $2.33 \mathrm{~mJ} / \mathrm{cm}^{2}$. Time scale is shorlened by increased rate constants. 


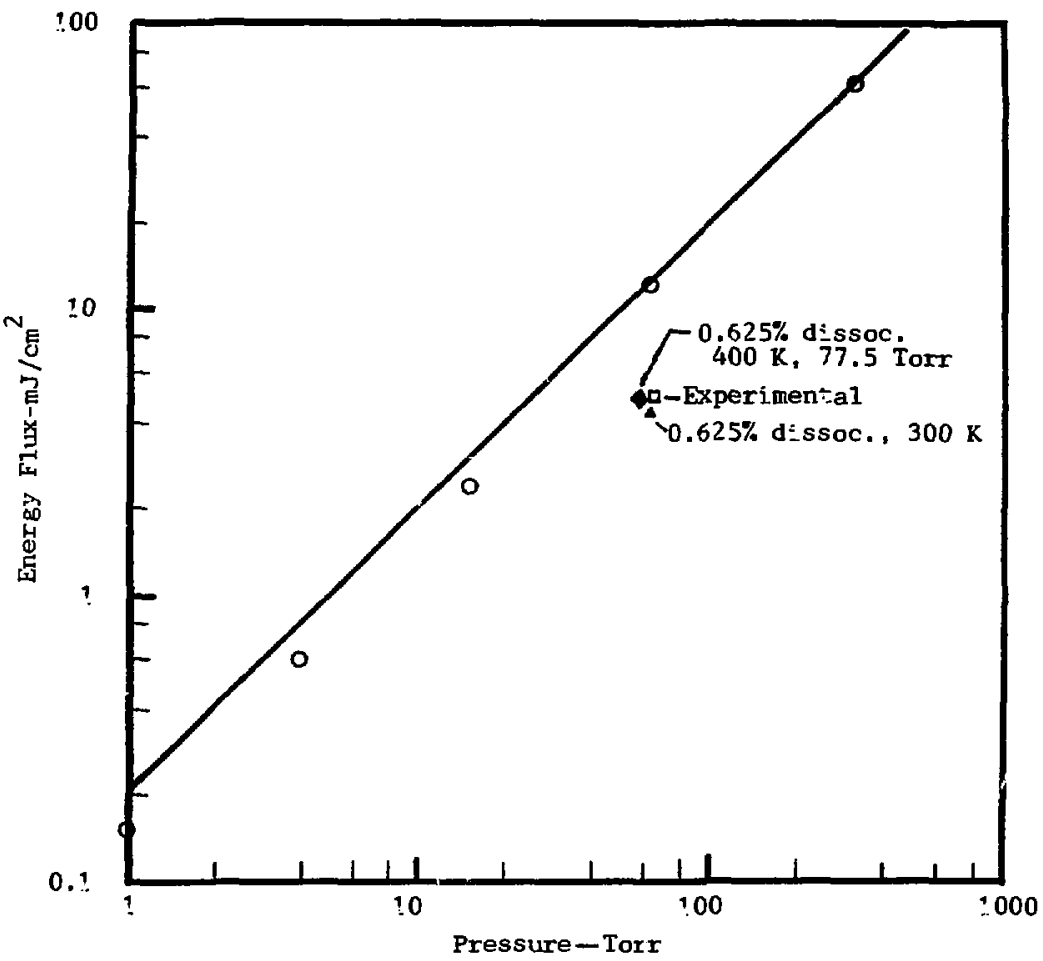

Fig. 9.96 Calculated total laser output energy as a function of total pressure for a ratio $\mathrm{NF}_{3} / \mathrm{H}_{2}=5.2$. Dissociation is $1.25 \%$ at $300 \mathrm{~K}$. The straight 1 ine is a linear $f: t$ at high pressure. 

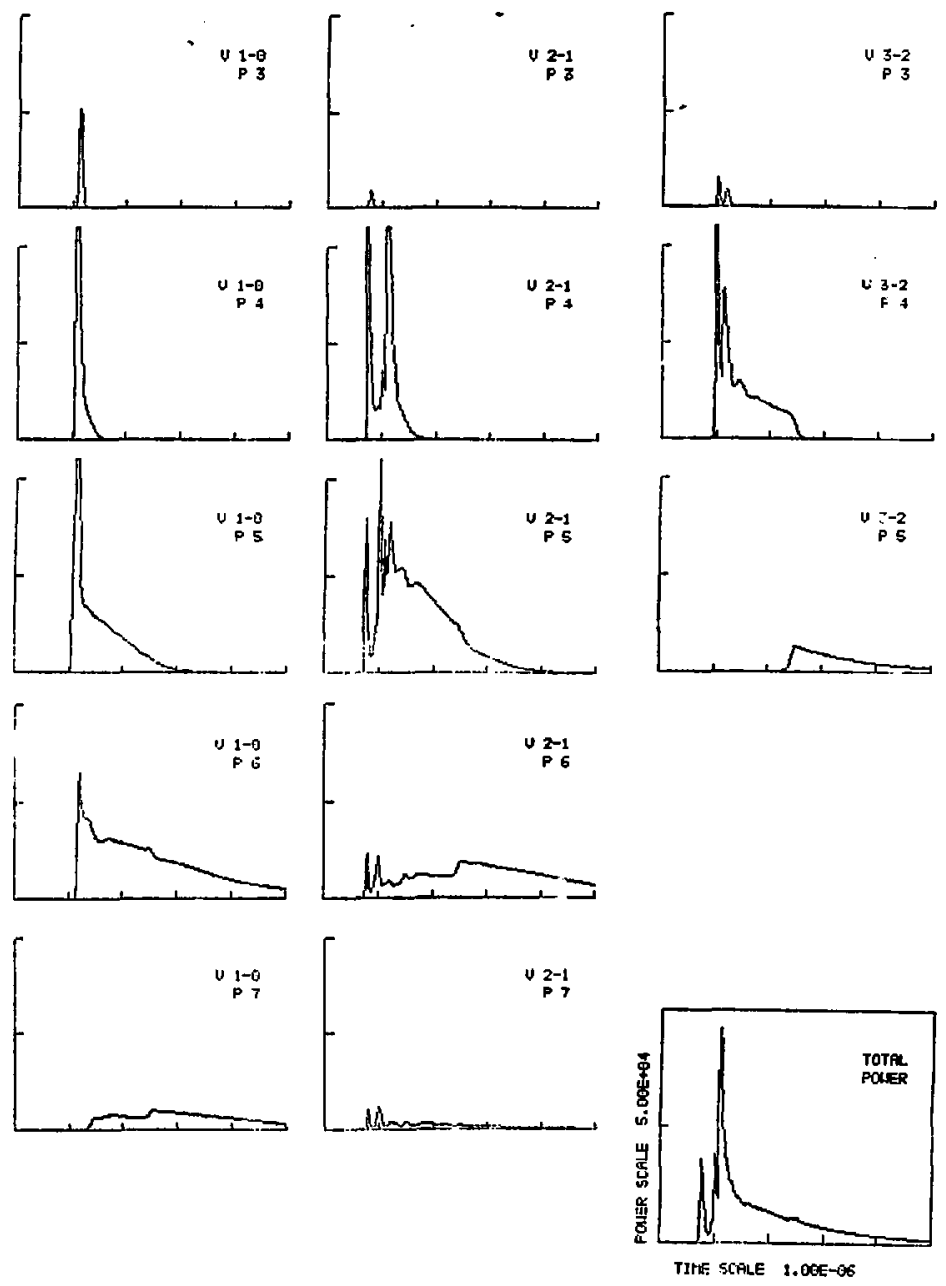

Fig. 9.17 Calculated waveforms at 62 Torr when dissociation is $0.6 \%$ at $300 \mathrm{~K}$. Total energy is $4.5 \mathrm{~mJ} / \mathrm{cm}^{2}$. 


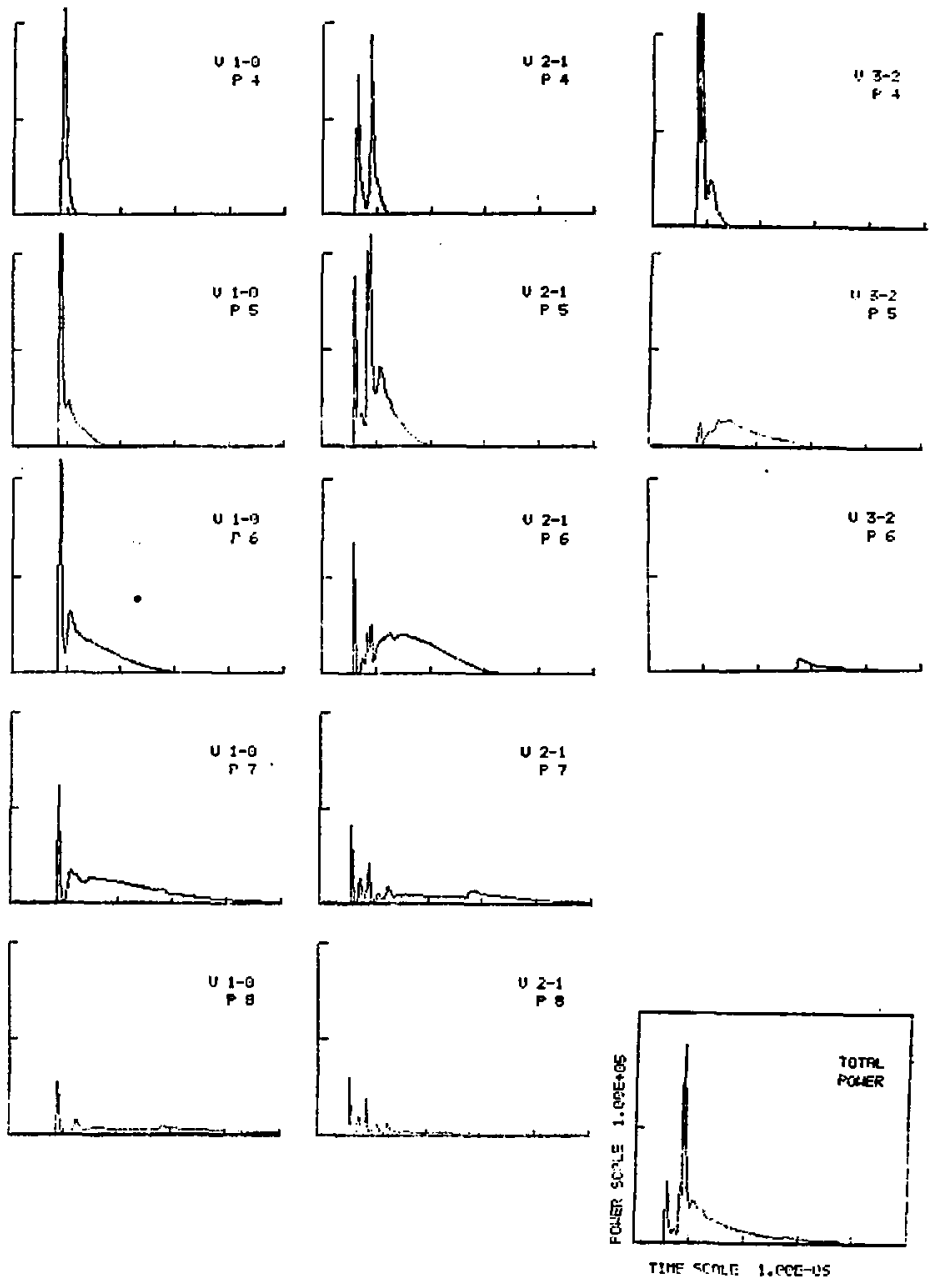

Fig. 9.18 Calculated waveforms at $400 \mathrm{~K}$ and 77.5 Torr (same density as $300 \mathrm{~K}, 58$ Torr), with $0.6 \%$ dissociation. Total energy was $5.0 \mathrm{~mJ} / \mathrm{cm}^{2}$. Laser intensity on P3 transitions was infinitesimal. 

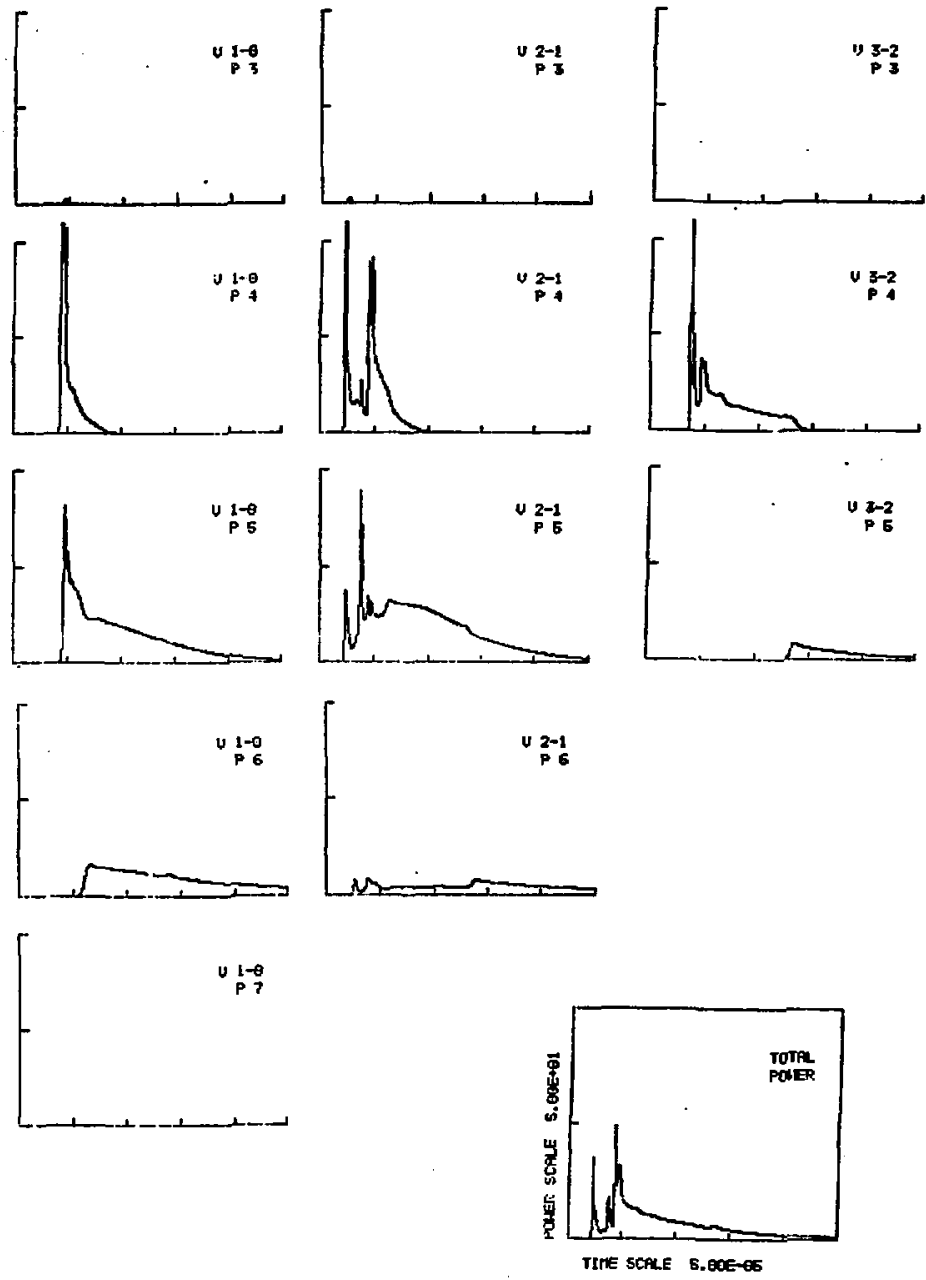

Fig. 9.19 Calculated waveforms at 1 Torr and $300 \mathrm{~K}$. Dissociation is $1.25 \%$, total energy is $0.15 \mathrm{~mJ} / \mathrm{cm}^{2}$. 


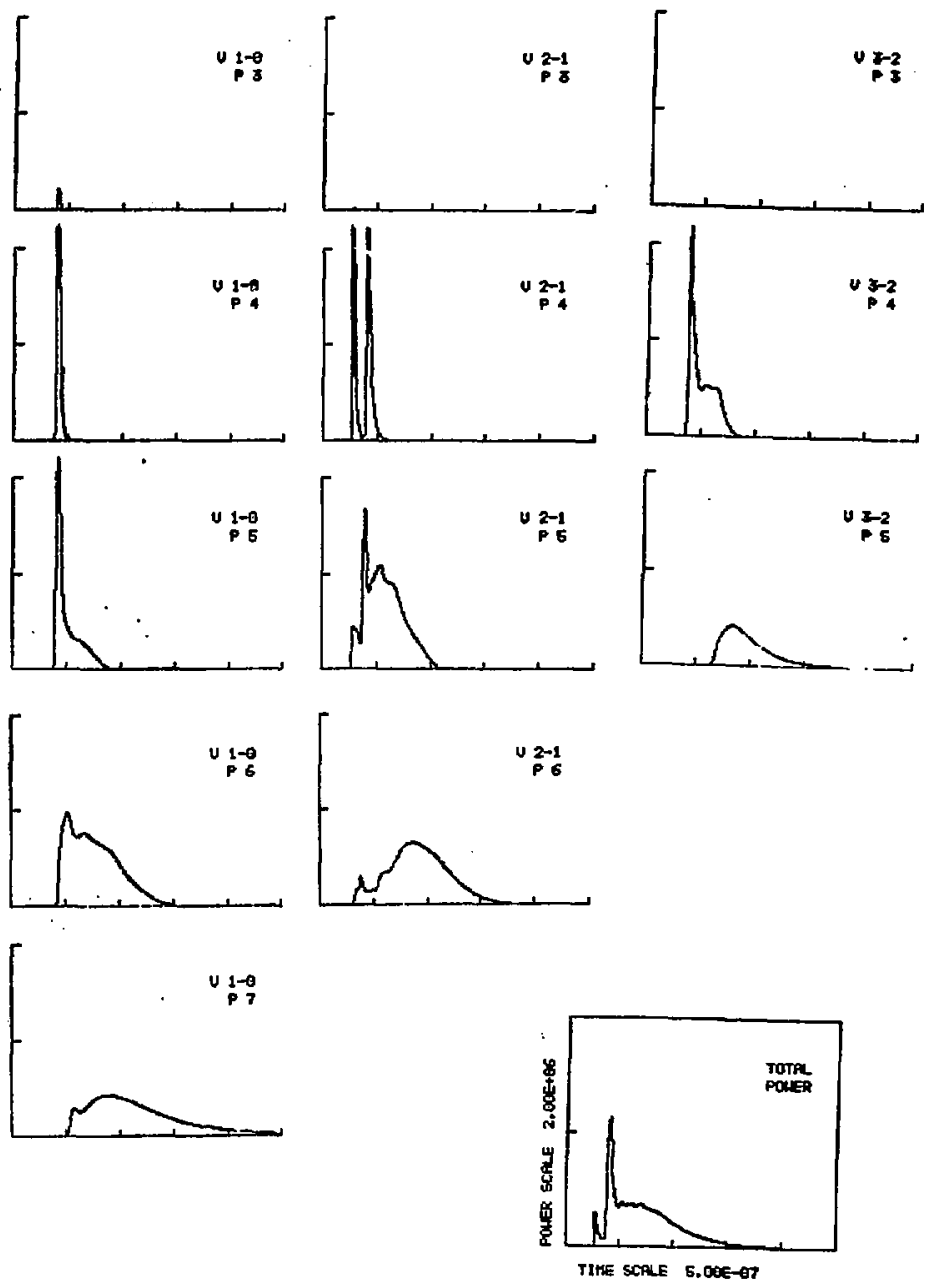

Fig. 9.20 Calculated waveforms at 310 Torr and $300 \mathrm{~K}$. Dissociation is $1.25 \%$, total energy is $64.3 \mathrm{~mJ} / \mathrm{cm}^{2}$. 
10. CONCLUSION

The discussion of the pulsed HF chemlcal laser has included two broad topics: modeling of the chemical kinetics and modeling of the laser action with rotational relaxation effects included.

The chemical kinetics accompanied by a steady state laser model was sufficient to determine the total laser waveform. With a steady state laser model the output power is proportional to the rate of formation of $\mathrm{HF}$, so calculation of the laser waveform is reduced to a kinetics calculation. With simplifying assumptions, including constant temperature, a simple analytic model can calculate the rate of formation of $\mathrm{HF}$. This can be done for the reaction $\mathrm{F}+\mathrm{H}_{2} \rightarrow \mathrm{HF}+\mathrm{H}$ and for the chain reaction of $\mathrm{H}_{2}+\mathrm{F}_{2}$. To obtain exact solutions of the kinetics, including temperature variation, the MINOTAR computer code was developed. A severe computational problem, the solution of "stiff" differential equations, was overcome by using a numerical technique developed by C. W. Gear (1971a). The exact numerical calculations verify the results of the analytic model under conditions where the temperature change is small. The MINOTAR code is also a useful tool for investigating more complicated kinetics problems such as those involved in combustion and air pollution.

In order to calculate the waveforms of individual P-branch transitions a better laser model was needed. The model treated each vibrational-rotational state seperately 
with adjacent rotational states coupled by rotational relaxation. In addition the model used semi-classical laser equations so that transient effects in the laser waveforms could be correctly calculated. Earlier models (Chester, 1970; Emanuel, 1971) assumed rotational equilibrium within each vibrational level and used a steady state laser model. The assumption of rotational equilibrium causes these models $: 0$ calculate a single P-branch transition lasing between two vibrational levels at any instant. The rotational levels must be partially de-coupled in order to calculate simultaneous lasing on several transitions, in agreement with experiment. The steady-state laser model ignores spiking behavior in the laser waveforms and grossly underestimates the time delay to the beginning of the laser pulse. The new model, incorporated in the IXION code, gives good agroenent with experiment. Simultaneous lasing on several $P$-branch transitions is seen and individual calculated P-oranch waveforms are quite similar to experimental waveforms. The calculated waveforms show spiking which explains the jagged nature of the experimental waveforms. The calculated time delays and pulse durations are approximately the same as those seen experimentally. Because the calculations agree with experiment they can be used to help interpret experiment. In particular, the time: histories of the populations and inversions are readily 1.ailable from the calculations but would be difficult to whain experimiental ly. 
The new model showed that our knowledge of the rotational rolaxation rates is inadequate. Recent experimental data (Peterson, 1974) indicates that collisional 1 ine broadeninp in $\mathrm{HF}$ may be entirely due to rotational relaxation, so that it inay be possible to determine rotational relaxation rates from line broadening experiments. These rates are an order of magnitude laster than rotational relaxation rates inferred from ultrasonic measurements. No serious attempt has yet been made t.o resolve this discrepancy.

Because of the limited experimental data available lor rotational relaxation rates an attempt was made to calculate the rates. This is presented in Appendix D. The physical model appears to be qualitatively correct but the quantitative results are not adequate. This deserves further examination and comparison with 1 ine broadening theories which give quantitatively correct results.

The new model has not been applied to the $\mathrm{H}_{2}+\mathrm{F}_{2}$ chain reaction laser. One would expect the model to show simultaneous lasing on several transiticns, and spiking behavior in laser waveforms similar to that seen in the $\mathrm{F}+\mathrm{H}_{2}$ laser. However, one would expect differences because the longer pulse would make vibrational relaxation important and would cause the rotational distributions to be nearer to a Bolt;uann equilibrium.

lt would be desirable to compare these calculations with an experiment which didn't involve the uncertainties of electric discharge physics. The ideal experiment would be 
one whers $F$ atoms were produced by photodissociation with a short laser pulse. Photodissociation by a flashlamp would be slower than the chemical reaction except at very low pressures; and the laser waveform would be nearly the same as the flashlamp waveform. In a properly designed experiment the initial density of $F$ atoms could be determined and the active volume accurately known. There would probably be some discrepancies between the results of such experiments and calculations. Attempts to climinate the discrepancies should lead to further insight into the physics of the laser. 
ACKNOHLJUGHENTS

The author wishes to acknowledge the holp of several people: Rober Harrach and Arnold karo for continued guidance and supporli N. L. Rapapnanj for romparison calculat ions using the NEST and RESALE codes; $A$ lan Hindmarsh and Robert Gel inas for assistance with tho Gear Technique; Alex Cecil Inr programming techniques; David Gregk, Richard Pearson, Gurton Herminn, Ethan Huss, and John Cowles for discussions ol experjmental results; Bill Curry, Ray Kidder, Bernard I i ppmanth, and Montgomery Johnson for discussions of the theory. 


\section{REFERENCES}

Milton Abramowitz and Jrone A. Stegun, (1964) "Handbnok of Mathematical Functions." U. S. Gov't., Natl. Bur. Stds.AMS -55 .

J. Richard Airey, $(1 / 70)$ "C1 + $\mathrm{HBr}$ Pulsed Chemical Laser:

A Theoretical and Experimental Study." J. Chem. Phys. 52. $156-167$.

J. R. Airey and S, F. Fried, (1971) Chem. Phys. Letters $\underline{8}$, 23.

P. W. Anderson, (1949) Phys. Rev, 76, 647.

K. G. AnJauf, P, J. Kuntz, D. H. Maylotte, P, D. Pacey, and J. C. Polanyi, (1967) "Energy Distribution Among Reaction Products Part 2. $\mathrm{H}+\mathrm{X}_{2}$ and $\mathrm{X}+\mathrm{HY} . "$, Disc. Faraday Sor. 44. 183 .

K. G. Anlauf, D. S. Horne, R. G. Macdonald, J. C. Polanyi, and K. B, Wooda11, (1972) "Energy Distribution Among Reaction Products. V. $\mathrm{H}+\mathrm{X}_{2}(\mathrm{X}=\mathrm{C} 1, \mathrm{Br}), \mathrm{b}+\mathrm{Cl}_{2} \cdot$, J. Chem. Phys. 57, 1561

Garrett Birkhoff and Saurders MacLane, (1965) A Survey of Modern Algebra, 3rd Ed. The MacMillan Co., N. Y., p. 107. N. Bloembergen and Y, R. Shen, (1964) "Quantum Theoretical Comparison of Nonlinear Susceptitilities in Parametric Media, Lasers, and Raman Lasers," Phys. Rev. 133, A37. ․ F. Bott and N. Cohen, (1971) "HF Vibrational Relaxation by F. Atoms." J. Chem. Phys. 55, 5124. 
J. de Boer and G. E. Uhienbeck, (1964) "Hrat Condurtivity and Viscosity." Studies in Statistical Mechanics, Vol. I1, 243-269, No. Holland-Interscience, New York.

C. A. Brat1 and R. M Jonkman, (1970) "Classical Theory of Rotational Relaxation in Diatomic Gases." J. Chem. Phys. 52, 477 .

R. Brout, (1954) J. Chem. Phys. 22, 934; J. Chem. Phys. 22, 1189 .

Alex Ceci1, (1965) "General Purpose Input Routines." CIC Report M-012, Lawrence Livermore Laboratory. Hao-Lin Chen and C. B. Moore, (1971) J. Chem. Phys. $\underline{54}, 4072$. A. N. Chester, (1970) "'Complete' and 'Partial' Vibrational lnversion in Chemically Pumped Molecular Lasers.", J. Chem. Phys. 53, 3595-8.

Arthur N. Chester and Larerne D. Hess, (1972) "Study of the Hl: Chemical Laser by Pulse-Delay Measurements."

IEEE J. Quantum Electron. QE-8, 1-13.

N. Cohen, T. A. Jacubs, G. Emanuel, and R. L. Wilkins, (1969)

"Chemical Kinetics of Hydrogen Halide Lasers, 1. The $\mathrm{H}_{2}-\mathrm{Cl}_{2}$ system." Int. J. Chem. Kinet. 1 , 551-69.

N. Cohen, (1971) "A Review of Rate Coefficients for Reactions in the $\mathrm{H}_{2}-\mathrm{F}_{2}$ laser System," Aerospace Corp. TR-0172 (2779)-2; (Air Force Rept. No. SAMSO TR-72-23).

P. H. Corneil and V. V. Kasper, (1969) "Computer Simulation of the HC1 Explosion Laser." Digest 2nd Conf. Cinem. Mo1. Lasers, St. Louis, Mo., May 22-24; 1969; also IEEE J. Quantum Electron. QE-6, 170 (1970). 
J. R. Creighton, R. K. Pearson, J. O. Cowles, G. L. Hermann, and D. W. Gregg, (1972) "Kinetic Rates from Transverse Discharge Chemical Laser Systems." 3rd Conf. on Chem. \& Molecular Lasers, St. Louis, Mo., May 1972. Abstract in IEEE J. Quantum Electronics QE-9, p. 201.

John R. Creighton, (1974) "Population Dynamics of a Pulsed HF Chemical Lasex." $16 \mathrm{~mm}$ Color (silent) movie.

R. J, Cross, Jr, and R. G, Gordon, (1966) J, Chem. Phys. 45,3571 .

B. P. Curry and R. E. Kidder, (1972) "A Multi-level Pulsed Chemica1 Laser Mode1.", Lawrence Livermore Laboratory, UCRL 74024 , rev. 1 .

B. P. Curry, S. A. Zwick, and C. D. Aliprantis, (1973) "ClosedForm Solution for Excited-State Populations in Temporally and Spatially Varying L.jer Media." Appl, Phys. Letters $\underline{23}, 574$.

L. W. Davis, (1963) "Semiclassical Treatment of the Optical Maser." Pros. IEEE $\underline{51}, 76$.

Janet E. Del Bene and J. A. Pople, (1971) "Theory of Molecular nteractions. II. Molecular Orbital Studies of $\mathrm{HF}$ Polymers Using a Minimal Slater-Type Basis." J. Chem. Phys, 55, 2296.

T. A. Dillon and J. C. Stephenson, (1973) "Calculation of Vibrational and Rotational Energy Transfer between $\mathrm{Hr}$, $\mathrm{BF}, \mathrm{HC}: 1$, and $\mathrm{CO}_{2} \cdot " \mathrm{~J}$. Chem. Phys. 58, 2056-2064. Hrigitta M. Dobratz, (1972) "Chemical Lasers: An Overview of the Literature.", 1960-71 Lawrence Livermore Laboratory UCRL-51285. 
Thomas R. Dyke, Brian J. Howard, and William Klemperer, (1972) "Radiof requency and Microwave Spectrum of the Hydrogen Fluoride Dimer; a Nonrigid Molecule." J. Chem. Phys. 56. 2442 .

G. Emanuel, (1964) "Numerical Analysis of Stiff Equations." Aerospace Corporation, El Segundo, Ca., Report SSDTI)R-63-380.

George Emanuel, (1971) "Analytic Model for a Continuous Chemical Laser." J. Quant. Spectrosc. Radiat. Transter 11, 1481-1520.

George Emanuel and James S. Whittier, (1972) "Closed-Form Solution to Rate Equations for an $\mathrm{F}+\mathrm{H}_{2}$ Laser Oscillator." Appl. Optics, Vol. I1, No. 9, 2047.

M. S. Feld and A, Javan, (1969) "Laser-Induced Line-Narrowing Effects in Coupled Doppler-Broadened Transitions." Phys. Rev, 177, 540 .

E. V. Franck and F. Meyer, (1959) "Flourwasserstoff III, Sperilische Warme und Assoziation im Gas bei niedrigem Druck." Zeits. Fur Electrochemie 63, 571 .

C. W. Gear, (1971a) "The Automatic Integration of Ordinary Differential Equations," Communications of the ACM 14, 176 . "Algorithm 407: DIFSUB for Solution of Ordinary Differential Equations [D2]." Comminication ACM 14, 185.

C. W. Gear, (1971b) Numerical Initial Value Problems in Ordinary Differential Equations. Prentice-Ha11, Englewood Cljiffs, N. J. 
H. Haken, R. der Agobian, and M. Pauthier (1965) Phys, Rev, 140A, 437.

H. Haken, (1970) Handbuck der Physik, "Light and Matter." Vol. 25, 259-267, Springer-Verlag, Berlin, Gottingen, Heidelberg.

G. Hancock and I. W. M. Smith, (1971) 3. Appl. Opt. 10, 1827 .

J. K. Hancock, and W. H. Green, (1972) "Laser Excited Vibrational Relaxation Studies of Hydrogen Fluoride." J. Chem. Phys, 트, 2474.

Gerhard Herzberg, (1950) Molecular Spectra and Molecular Structure: I. Spectra of Diatomic Molecules. D. Van Nostrand, Princeton, N, $\mathbf{3}$.

Karl F. Herzfeld and Theodore A. Litovitz, (1959) Absorption and Dispersion of U1trasonic Waves. Academic Press, New York, 260-352.

A. C. Hindmarsh, (1972a) "GEAR: Ordinary Differential

Equation System Solver." U. C. Lawrence Livermore Laboratory, UCID-30001, Rev. 1 .

A. C. Hindmarsh, (1972b) "Construction of Mathematical

Software, Part 1II: The Control of Error in the Gear Package for Ordinary Dj.fferential Equations." U. C. Lawrence Livermore Laboratory, UCID-30050, Pt. 3.

A. C. Hindmarsh, (1973) "GEARB: Solution of Ordinary Uifferential Equations Having Banded Jacobian." U. C. Lawrence Livermore Laboratory, UCID-30059. 
J. J, I. Hough and R, L., Kerber, (1274) "Theoretisal Investigation of the Effect of Rotational Nonequilibrium on Predicted Performance of Pulsed HF Chemical Lasers." 4th Conf. Chem. \& Mol. Lasers, St. Louis, Mo, Oct. 1974. Paper TA7, Abstract in IEEE J. Quantum Electronics, QE.11, 699. (1975)

V. 1. Igoshin, L. V. Kulakov, and A. 1, Nitkin, (1973) "Deiermination of the Rate Constant of the Chemical Reaction $\mathrm{F}+\mathrm{H}_{2}\left(\mathrm{D}_{2}\right)-\mathrm{HF}(\mathrm{DF})+\mathrm{H}(\mathrm{D})$ From the Stimulated Enission of the HF(DF) Molecules." Kvant. Electron. $\underline{5}$, 50-59; (trans1. Sov. J. Quant. Electron 3, 306-311 (1974).)

Ali Javain, (1957) "Theory of a Three-Level Maser." Phys. Rev. 107. 1579.

R. Jensen, (1973) Los Alamos Scientyfic Laboratory

Private communication.

William Klemperer, (1972) Harvard University

Private Communication.

Peter A. Kollman and Leland C. Allen, (1970) "Theory of the Hydrogen Bond: Ab Initio Calculations on Hydrogen Fluoride Dimer and Mixed Water-Hydrogen Fluoride Dimer." J. Chem. Phys. 52, 5085.

J. D. Lambert, (1962) "Relaxation in Gases," Chpt. 20, Atomic and Molecular Processes, D. R. Bates, Ed., Academic Press, N. Y.

L. Landau and E. Teller, (1936) Physik 2. Sowjetunion 10. 34 .

William H. Louise11, (1964) Radiation and Noise in Quantum Electronics. McGraw-Hil1 Book Co., New York, p. 75. 
George Makhov, (1962) "On the Problem of Pulsed Oscillations in a Ruby Maser." J. App1. Phys. 33, 202.

D. E. Mann, B. A. Thrush, D. R. Lide, Jr., J. J. Ba11, and N. Acquista, (1961) "Spectroscopy of Flourine Flames I. Hydrogen-Flourine Flame and the Vibration-Rotation Emission Spectrum of HF." J. Chem. Phys. 34, 420-431. Andrew J. Matheson, (1971) Molecular Acoustics, WileyInterscience, N. Y., pp. 47-76. D. H. Maylotte, J. C. Polanyi, and K. B. Woodall, (1972) "Energy Distribution Amcng Reaction Products IV. $\mathrm{X}+\mathrm{HY}(\mathrm{X}=\mathrm{Cl}, \mathrm{Br}, ; \mathrm{Y}=\mathrm{Br}, \mathrm{I}), \mathrm{Cl}+\mathrm{D} 2 . " \mathrm{~J}$. Chem。 Phys. 57, 1547.

R. E. Meredith, (1972) "Strengths and Widths in the First Overtone Band of Hydrogen Fluoride." J. Quant. Spectrosc. Radiat. Transfer 12, 485-503. Joseph S. Murphy and James E. Boggs, "Collision Broadening of Rotational Absorption Lines." (in 4 parts)

I. Chem. Phys, 47, 691-702 (1967)

J. Chem. Phys. 47, 4152-4158 (1967)

J. Chem. Phys. 49, 3333-3343 (1968)

J. Chem. Phys. 50, 3320-3329 (1969)

Carl Nyeland, (1967) "Rotational Relaxation of Homonuclear Diatomic Molecules," J. Chem. Phys. 46, 63. T. D. Padrick and M. A. Gusinow, (1974) "Evidence of Rotational Nonequilibrium in the $\mathrm{CH}_{3} \mathrm{I}+\mathrm{N}_{2} \mathrm{~F}_{4}$ Chemical Laser." Chem. Phys, Letters 24, 270. 
Richard H. Pantell and Harold E. Puthof', (1969)

Fundamentals of Quantum Electronics. John Wiley \& Sons, Ine., N. Y.

J. G. Parker, (1959) "Rotational and Vibrational Relaxation in Diatomic Gases." Phys. Fluids 2, 449.

Richard K. Pearson, J. O. Cowles, G. L. Hermann, K. J. Pettipiece, and b. W. Gregg, (1973a) "Pressure Dependency of the $\mathrm{NF}_{3}-\mathrm{H}_{2}$ Transverse-Discharge Pulse Initiated HF Chemical Laser." IEEE J. Quant. Electronics QE-9, 723-730.

Ri.chard K. Pearson, J. O. Cowles, G. L. Hermann, D. W. Gregg, and J. R. Creighton, (1973b) "Relative Performance of a Variecy of $\mathrm{NF}_{3}+$ Hydrogen-Donor Transverse-Discharge HF Chemical- Laser System." IEEE J. Quant. Electronics QE-9, 879-889.

L. M. Peterson, G. H. Lindquist, and C. B. Arnold, (1974) "Rotational Relaxation Measurements of Laser-Excited Hydrogen Fluoride." J, Chem. Phys. 61, 3480.

J. C. Polanyi and K. B. Woodal1, (1972a) "Mechanism of Rotational Relaxation." J. Chem. Phys. 56, No. 4, 1563.

J. C. Polanyi and K. B. Wooda11, (1972b) "Energy Distribution Among Reaction Products: VI. $\mathrm{F}+\mathrm{H}_{2}, \mathrm{D}_{2} \cdot "$ J. Chem. Phys. 57, 1574 .

J. C. Polanyi and J. J. Sloan, (1972) "Energy Distribution Among Reaction Products: VII. $\mathrm{H}+\mathrm{F}_{2} \cdot " \mathrm{~J}$, Chem. Phys. 57, 4988. 
H. Pummer and K, L. Kompa, (1972) "Investigation of a $1-\mathrm{J}$ Pulsed Di.scharge-Initiated HF Laser." Appl. Phys. Lett. 20, 356 .

L. M. Raft and T. G. Winter, (1968) "Origin of the Temperature Dependence of the Ultrasonic Rotational Relaxation Time." J. Chem. Phys, 48, 3992 .

N. L. Rapagnani, (1972) "The Effects of Various ChainBranching Rates on the Pulsed Hl Chemical Laser." Lawrence Livermore Laboratory UCID-16006 (Unpub. internal report)

N. L. Rapagnani, (1973)

Private communication.

Donald Rapp, (1960) "Complete Classical Theory of Vibrational Energy Exchange." J. Chem. Phys. 32, 735.

Donald Rapp and Thomas Kassal, (1969) "The Theory of Vibrational Energy Transfer Between Simple Molecules in Non-reactive Col1isions." Chem. Rev, 69, 61. H. Risken and K. Nummedal, (1968) "Self-Pulsing in Lasers." J. Appl. Phys, 39, 4662 .

Norman F. Sather and John S. Dahler, (1961) "Molecular Friction in Dilute Gases." J. Chem. Phys. 35, 2029, J. Chem. Phys. 37, 1947 (1962).

John H. Seinfeld, Leon Lapidus, and Myungkyu Hwang, (1970)

"Review of Numerical Integration Techniques for Stiff Ordinary Differential Equations." Ind. Eng. Chem. Fundam, 9, 266. 
1.. H. Sentman, "Rotall ional Nonequilibrium in ew Chemical lansers." ith Conf. on Chem. \& Mol, Lasers, St. Louis, Mo., Oct. 1974, Paper T1)7. Abstract in [F.F. J. Quantum Electronics QE11, 706.

H. K. Shin, (1970)

Chenl. Phys, Lotlers 6 , 494

H. K. Shijn, (1971)

Chem. Phys. Letters 10, 81.

H. K. Shin, (1972) "Tenperature Dependence of the Probability of Vibrational Energy Transfer Between HF and F." Chem. Phys. I,ctters $14,64$.

Jon H. Shirley, (1968) "Dynamics of a Simple Maser Model." AnI. J. Phys. 36, 949.

L. M. Sinnett, (1962) "An Analysis of Maser Oscillator Eqquatjons." J. Appl. Phys. 3ㅜ, 1578.

D. F. Smith, (1958) "Hydrogen-Fluoride Polymer Spectrum, Hexamer and Tetramer." J. Chem. Phys. 28, 1040.

1). F. Smith, (1959) "The Overlapping Hydrogen-Fluoride Mononer-Dimer Spectra." J. Mol. Spectrosc. $\underline{3}$, 473-485.

I. C. Solomon, J. A. Blauer, F. C. Jaye, and J. G. Huat, (1971) Intern. J. Chem Kinetics 3, 215.

Donald L. Thompson, (1972) "Mechanisms for Hydrogen Halide Vibrational Relaxation." J. Chem. Phys. 57, 2589.

A. $\because$ Trotman-Dickenson and G. S. Milne, (1967) Tables of Bimolecular Gas Reactions, NSRDS-NBS9, U,S. Gov't., Nat'1 Bur, Stds, Washington, D.C. p. 15. 
C. J. Tsao and B. Curnutte, (1962) "Line-Widths of Pressure Broadened Sprotral linos." J, Quant. Spectrosc. Radiat. Transfer 2, 41-91.

H. W. Turnbtull, (1957) Theory of Equations, Oliver \& Boyd, Edinburgh \& London. pp. 95ff.

Emily B. Turner, W. Dallas Adams, and George Emanue1, (1973) "Numerical Formulation for Constant-Gain Chemical Laser Calculations." J. Comput. Phys. 11, 15-27.

A. von Engel,(1956) "lonization in Gases by Electrons in Electric Fields," Handbuch der Physik, Vol. XXI, Electron-Emission Gas Discharges I, Springer-Ver?ag, Berlin. pp. 504-573.

B. Widom, (1960) "Rotational Relaxation of Rough Spheres." J. Chem. Phys. 32, 913. Frank J. Zeleznik, (1967) "Rotational Relaxation in Polar Gases." J. Chem. Phys. 47, 3410. A1so see J. Chem. Phys. 53, 632 (1970); and J. Chem Phys. 54, 4523 (1971). 
Appendix A - Vibrational-Rotational Spoctrum of Hydrogen flourido

Tho following tables give the calculated spectrum of HF. Chr calculation is based on a Dunham expansion us ing co-eificients from Mann, et a1. (1961). These accurate co-efficients are obtained by a curve fit to extensive experimental data. The values of the co-efficients are givel: in [ablo A], alomg with the equivalent symbol in Herzberg's notation (1950). (There is a minor correction to get from Dunham co-efficients to Herzberg's.) Table A2 gives HF vibrational rotational term values up to $v=9$ and $J=19$. Table A3 gives the vacuum wavelength and wavenumber of the $P$ and $R$ branches of importance of the HF laser. These are accurate to ibout $0.1 \mathrm{~cm}^{-1}$. 
Table A1 - Dunham co-efficients used to calculate the spectrum of HF. They are equivalent, wit: a small correction, to the symbols used by Herzberg.

\begin{tabular}{|c|c|c|}
\hline \multicolumn{2}{|c|}{ Symbol } & \multirow[t]{2}{*}{ Value } \\
\hline Dunham & Herzberg & \\
\hline $\mathrm{Y}_{\mathrm{OO}}$ & $\mathrm{Te}$ & 0.0 \\
\hline$Y_{10}$ &.$_{\mathrm{e}}$ & 4138.73 \\
\hline$Y_{20}$ & $-w_{e} x_{e}$ & -90.05 \\
\hline $\mathrm{Y}_{30}$ & $w_{e}^{y_{e}}$ & 0.932 \\
\hline$Y_{40}$ & $-w^{z} e$ & -0.0142 \\
\hline$Y_{50}$ & - & -0.00059 \\
\hline$Y_{01}$ & $B_{e}$ & 20.9555 \\
\hline $\mathrm{Y}_{11}$ & $-\alpha_{e}$ & -0.7958 \\
\hline $\mathrm{Y}_{21}$ & $\gamma_{\mathbf{e}}$ & 0.01182 \\
\hline $\mathrm{Y}_{31}$ & - & -0.000311 \\
\hline$Y_{41}$ & - & -0.0000058 \\
\hline $\mathrm{y}_{02}$ & $-D_{e}$ & -0.002153 \\
\hline$Y_{12}$ & $\beta_{e}$ & 0.0000623 \\
\hline$Y_{22}$ & - & -0.00000206 \\
\hline $\mathrm{Y}_{03}$ & $\mathrm{H}_{\mathrm{e}}$ & $1.068 \times 10^{-7}$ \\
\hline $\mathrm{Y}_{13}$ & - & $-6.5 \times 10^{-9}$ \\
\hline$Y_{04}$ & - & $-1.9 x+y^{-11}$ \\
\hline
\end{tabular}




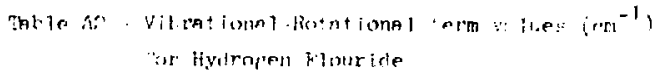

HYDROFEN FLOURIIF VIBFATIONML-ROTATIC TIL TEKMS (IFCM

$\mathbf{V}=\mathbf{B}$

9562.14

8836.75

B 144.31

7485.68.

$6861 \cdot 46$

6272.57

$\$ 719.65$

520.37

4724.35

A28. 3.16

3860.34

3516.38

3191.73

2906.78

2661.88

$2457 \cdot 33$

2293. 39

2170.25

2088.48

2046.97 $v=1$

13237.93

I 2SA4. 45

11874.56

$11 \div 41.07$

10640.74

1097A. 29

$9542.4 P$

9045.73

658 4.87

8160.39

$7772 \cdot 80$

7422.60

7114.20

$6 B 35.99$

6600.32

6403.47

6245.70

6127.20

$6048 \cdot 17$

6008.55 $v=2$

$167 \triangle 8.85$

16678.56

15438.57

14829.66

14252.56

13707.99

13196.60

12719.64

12275.87

11867.67

11494.92

11158.10

16857.63

10593.89

16367.20

10177.86

16026.09

9912.10

9836.42

9797.96 $v=3$

$20098.7 \mathrm{~K}$

19455.11

188 a9. 45

18255.57

17701.19

17177.99

16686.63

16227.12

15861.84

15409.52

15ab1.26

| 4727. 51

$14438.6 B$

14185.15

13967.22

13785. 19

13639.29

13529.79

13456.55

13419.96
$v=\Delta$

23291.13

2.2673 .57

2РЯК3.75

21522.43

20990. 31

20488. 15

20016.31

19575.67

19365.71

18789.94

1R445.8A

16132.96

17857.46

17613.9

1740.50

17229.69

17689.51

16984.22

16913.95

16878.79

HYDROTFN FLQURIUE VJEFATIONAL-ROTPTIONAL TEFNS (I/CM)

$$
\mathbf{v}=\mathbf{5}
$$

$26328 \cdot 61$

25736.75

25171.38

24633.23

24122.99

23641,32

$23188 \cdot 84$

22766.16

22373.61

22012.32

21682.15

$213 B 3.74$

$21117.4 B$

29863.73

26682. 79

20534.93

203R日. 38

26279.31

20211.85

$2017 \mathrm{~B}+10$ $v=6$

29213.25

28646.78

28105.54

27596.26

27193.61

26649.2 ด

$26206 \cdot 77$

25841.78

25425.81

25879.36

24762.89

24476.R5

2A221 - 69

23997.49

23804.83

23643.89

23514.86

23417.94

23253.25

20310.89 $v=7$

31946.29

31405.00

39827.68

39395.85

29927.79

29486.52

29071.86

28684.38

28324.60

27993.03

27690.12

27416.29

27171.92

26957.35

26772.67

2651B. 75

26495.19

26 ABD. 37

PG341. A2

26309.42 $v=8$

34528.12

34011.89

33518.39

33548 . 31

32RA2. 31

$32,81.03$

31765.06

31414.97

31071.28

30754.49

30465.03

30203.33

29969.76

29764.64

295AB. 28

29446.92

29328. 79

29234.03

29174.79

29145.15 $v=9$

$36958 \cdot 18$

36467.64

35997.34

35549.79

35125.04

34723.72

343A6. 42

33993.69

33666.95

33363.99

33087.95

32838.34

32615.53

$32+19+B 4$

32251.56

32110.95

$31998 \cdot 21$

31913.51

31856.97

31828.68 
Table A3 - P and R Eranch Trensitions in Hydroren Flouride

HYDROGEN FLOURIDE SPECTRUM FOR VIBRATIONAL-ROTATIONAL TRANSITIONS FROM $V=$ TO VE $O$

P BRANCH
WAUE NUMBER
WAVELENGTH
(I/CN)
(MICRONS)

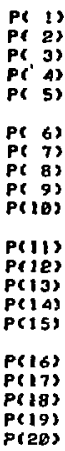

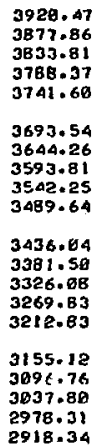

A BRANCH NAVE NLABER (1/CH)

Re D)

Rर 1 )

R( 2)

Re 3)

R( A)

R( 5$)$

Re 65

RC 7$)$

R( S)

R( क)

ReIGs

R(1)

R(12)

R(13)

$R(14)$

R(15)

Re 16$)$

R(17)

R(18)

R(1?)

$\mathrm{R}(2)$

4001.15
4939.12
4975.45
4110.08
4142.99
4174.11
4263.42
4230.87
4256.42
4230.05
4301.71
4321.38
4339.43
4354.43
4368.17
4379.61
4388.94
4396.14
4491.20
4484.10
4404.83

VACULM

WAVELENGTH

(HICRONS)

$$
\begin{aligned}
& 2.49928 \\
& 2.47579 \\
& 2.45372 \\
& 2.43304 \\
& 2.41372 \\
& 2.39572 \\
& 2.37902 \\
& 2.36358 \\
& 2.34939 \\
& 2.33642 \\
& 2.32466 \\
& 2.31498 \\
& 2.30466 \\
& 2.29640 \\
& 2.28929 \\
& 2.28331 \\
& 2.27846 \\
& 2.27472 \\
& 2.27211 \\
& 2.27361 \\
& 2.27024
\end{aligned}
$$

HYDROGEN FLDURIDE SPECTRLM FOR VIRRATIONAL-ROTATIONAL TRANSITIONS FROM $V=2$ TO $V=1$

$\begin{array}{cc}\text { P BRANCH } & \text { VACUUM } \\ \text { WAVE NUMBER } & \text { WAVELENGTH } \\ \text { C I /CW } & \text { (MICRONS }\end{array}$

$\begin{array}{ll}P(1) & 3749.84 \\ P(2) & 3708.72 \\ P(3) & 3666.49 \\ P(4) & 3627.62 \\ P(5) & 3577.54 \\ P(4) & 3531.21 \\ P(7) & 3483.69 \\ P(8) & 3435.64 \\ P(9) & 3385.30 \\ P(19) & 3334.53 \\ P(11) & 3252.86 \\ P(12) & 3230.15 \\ P(13) & 3176.63 \\ P(14) & 3122.32 \\ P(15) & 3667.26 \\ P(16) & 3011.50 \\ P(17) & 2955.10 \\ P(18) & 2898.12 \\ P(19) & 2848.61 \\ P(20) & 2782.62\end{array}$

R BRANCH WAYE NUMBER $(1 / \mathrm{CH})$

$$
\begin{aligned}
& 3827.47 \\
& 3963.98 \\
& 3898.89 \\
& 3932.15 \\
& 3963.73 \\
& 3993.57 \\
& 4921.64 \\
& 4647.91 \\
& 4872.32 \\
& 4894.86 \\
& 4115.49
\end{aligned}
$$

Re 9 ) $R(10)$

3.64618
3.09584
3.14799
3.20275
3.26024

R(1)

R(I2)

$R(13)$

FC 1 a

R( 15$)$

3.32061

3. 38398

3. 45051

3. 52037

3.5937 A
RS16)

R(17)

R(1B)

Re 19$)$

R(20)
A) $34 \cdot 17$

4150.88

4165.59

$4178 \cdot 28$

4188.92

$4197.5 \theta$

$\triangle 204.60$

4208.49 4210.68 $4210 \cdot 85$
VACUUM WAVELENGTH (HICKONS)

$$
\begin{aligned}
& 2.61269 \\
& 2.58800 \\
& 2.55483 \\
& 2.54314 \\
& 2.52288 \\
& 2.58402 \\
& 2.48655 \\
& 2.47341 \\
& 2.45560 \\
& 2.44208 \\
& 2.42985 \\
& 2.41887 \\
& 2.48913 \\
& 2.48062 \\
& 2.39333 \\
& 2.38725 \\
& 2.38237 \\
& 2.37669 \\
& 2.37620 \\
& 2.37491 \\
& 2.37488
\end{aligned}
$$$$
\text { 2. } 374 \mathrm{BR}
$$ 
HYOROGEN FLOURIDE SPECTRLN FOR VIBRATIONAL-ROTATIONAL TRANSITIONS FROH $V=3$ TO $V=2$

P BRANCH
WAVE NUMBER WAUELENGTH
(1/CM)

$\begin{array}{lll}\text { P( 1) } & 3583.94 & 2.79623 \\ P(2) & 3544.46 & 2.82131 \\ P(3) & 3503.60 & 2.85420 \\ P(4) & 3461.43 & 2.88698 \\ P(5) & 3417.99 & 2.92569 \\ P(6) & 3373.34 & 2.96442 \\ P(7) & 3327.51 & 3.09525 \\ P(8) & 3280.58 & 3.04824 \\ P(9) & 3232.59 & 3.09350 \\ P(10) & 3183.59 & 3.14110 \\ & & \\ P(11) & 3133.65 & 3.19117 \\ P(12) & 3082.80 & 3.24386 \\ P(13) & 3031.12 & 3.29911 \\ P(14) & 2978.64 & 3.35724 \\ P(15) & 2925.43 & 3.41836 \\ P(16) & 2871.53 & 3.49246 \\ P(17) & 2817.00 & 3.54987 \\ P(18) & 2761.98 & 3.62876 \\ P(19) & 2706.26 & 3.69514 \\ P(29) & 2650.15 & 3.77338\end{array}$

\author{
R BRANCH \\ W.9L'? NLMBER \\ ( I ( CM)
}

ac 6$)$

Re is

Re 23

R( 3$)$

Re 4)

RY 5$)$

Re 6)

Re 7)

F( B)

R( 9)

R(1D)

R(1)

R(12)

R(13)

R( 14 )

RC15\}

$R(16)$

R(17)

R(18)

R(19)

R(20)

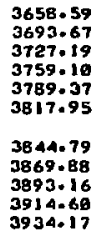

3951.85

3967.59

3981.39

3993.20

4003.61

4910.80

4016.54

4020.23

4621 - 84

4021 - 35
VACULA

WAVELENGTH

(HI CKONS)

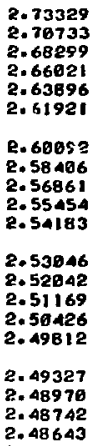

2. 48643

2. $4 B 672$

HYDADGEN FLOURIDE SPECTRLA FOR UIBFATIDNAL-ROTATIONAL

TRANSITIONS FKOH $V=$ A TO $V=3$

P BRANCK

WAVE NUMEER

(1/CW)

WAVELENE'TH

$\begin{array}{ll}P(1) & 3422.23 \\ P(2) & 3384.25 \\ P(3) & 3544.93 \\ P(4) & 3304.32 \\ P(5) & 3262.46 \\ P(6) & 3219.41 \\ P(7) & 3175.23 \\ P(8) & 3129.95 \\ P(9) & 3683.64 \\ P(10) & 3036.34 \\ P(11) & 2984.19 \\ P(12) & 2938.99 \\ P(13) & 2889.94 \\ P(14) & 2838.31 \\ P(15) & 2786.86 \\ P(16) & 2734.73 \\ P(17) & 2681.98 \\ P(18) & 2628.64 \\ P(19) & 2574.78 \\ P(20) & 2520.44\end{array}$

VACUUM

(HICFONS)

$R$ BRENCH WAVE NUHBEF (1)CNi)

$\begin{array}{ll} & R(\text { B) } \\ 2.92267 & R(1) \\ 2.95486 & R(2) \\ 2.98969 & R(3) \\ 3.92634 & R(4) \\ 3.66517 & R(5) \\ & \\ 3.10616 & R(6) \\ 3.14938 & R(7) \\ 3.19494 & R(B) \\ 3.24893 & R(9) \\ 3.89344 & R(10) \\ 3.34661 & R(11) \\ 3.48253 & R(12) \\ 3.46136 & R(13) \\ 3.52322 & R(14) \\ 3.58826 & R(15) \\ & \\ 3.65666 & R(16) \\ 3.72859 & R(17) \\ 3.88424 & R(18) \\ 3.88382 & R(19) \\ 3.96756 & R(20)\end{array}$

VACLILN

WAVELENGTH

(MICKONS)

2.86296

2.83474

2.80913

2.78521

2.76291

2. 74222

2. 72308

2.70547

2.68937

2. 67474

2. 66157

2. 64983

2. 63951

2. 63060

2. 62308

2.61695

2.61e21

2.60BB5

2.69687

2.60629

2.69710 
HYDROFEN FLOURIUF SPECTRUM FOR VIRRATIONAL-ROTATIONAL TRANSIJIOLS FHOM Vx $S$ TO Vx A

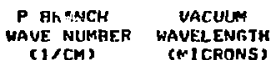

\begin{tabular}{|c|c|}
\hline $\begin{array}{ll}\text { P: } & 1 \\
\text { P: } & ? \\
\text { Pl } & 3 \\
\text { P( } & 4 \\
\text { P( } & 5\end{array}$ & $\begin{array}{l}3264.15 \\
328.7 .63 \\
3169.89 \\
3150.70 \\
3110.37\end{array}$ \\
\hline $\begin{array}{ll}\text { PC } & 6 \\
\text { PC } & 7 \\
\text { Pt } & \text { A } \\
\text { PC } & 9 \\
\text { PC } & \theta\end{array}$ & $\begin{array}{l}39 i 3.88 \\
3926.27 \\
2982.59 \\
9937.88 \\
2892.23\end{array}$ \\
\hline $\begin{array}{l}\text { PCI } 1 \\
\text { PC } 12 \\
\text { PC } 13 \\
\text { PC } 14 \\
\text { PSIS }\end{array}$ & $\begin{array}{l}2845.61 \\
2798.14 \\
8749.85 \\
2700.79 \\
2651.91\end{array}$ \\
\hline $\begin{array}{l}\text { PC } 16 \\
\text { PC } 17 \\
\text { PC } 18 \\
\text { PC } 19 \\
\text { PC } 29\end{array}$ & $\begin{array}{l}2400.55 \\
2549.47 \\
2497.81 \\
2445.62 \\
2392.95\end{array}$ \\
\hline
\end{tabular}

3.46356 3. B9B25

3. 13580

3. 17390

3.21505

3.25852

3. $39 \triangle 4 G$

3. 352R日

3. 49381

3. 45757

3. 51419

3. 57389

3.63656

3. 70242

3.77215

3.8A533

3. 92238

A. 90356

4.68894

$4+17894$

\author{
A BRANCH \\ HAVE NUMRER \\ (1/CM)
}

\begin{tabular}{|c|c|c|}
\hline $\begin{array}{ll}\text { RC } & \text { ब } \\
\text { RC } & 1 \\
\text { FC } & 2 \\
\text { RC } & 3 \\
\text { FC } & 4 \\
\text { RC } & 5\end{array}$ & $\begin{array}{l}3333.06 \\
3365.36 \\
3396.16 \\
3425.42 \\
3453.11 \\
3479.17\end{array}$ & $\begin{array}{l}3.9999 A \\
2.97145 \\
2.99450 \\
2.91935 \\
2.89594 \\
2.87425\end{array}$ \\
\hline $\begin{array}{ll}\text { RC } & 6 \\
\text { HC } & 7 \\
\text { RS } & \text { G } \\
\text { RC } & 9 \\
\text { RC } & 10\end{array}$ & $\begin{array}{l}3503.57 \\
3526.28 \\
3547.25 \\
3566.46 \\
3583.47\end{array}$ & $\begin{array}{l}2.65423 \\
2.43515 \\
2.81908 \\
2.80390 \\
2.79088\end{array}$ \\
\hline 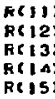 & $\begin{array}{l}3599.45 \\
3613.17 \\
3625.91 \\
3634.73 \\
3642.9 ?\end{array}$ & $\begin{array}{l}2.77820 \\
2.76765 \\
2.75851 \\
2.75198 \\
? .74505\end{array}$ \\
\hline $\begin{array}{l}\text { RCIG } \\
\text { RC } 17 \\
\text { HC18 } \\
\text { EC } 19 \\
\text { RC } 20\end{array}$ & $\begin{array}{l}3648.95 \\
2653.62 \\
3655.94 \\
3655.07 \\
3653.05\end{array}$ & $\begin{array}{l}2.74051 \\
2.73748 \\
2.73595 \\
2.73593 \\
2.73744\end{array}$ \\
\hline
\end{tabular}

VACUUY. WAVELFATTH (PICKONS)

HYDFOHEN FLOURIDE SPECTRUM FOF VIBKATIONAL-ROTATIDNAL TRANSITIONS FROM $V=670 \mathrm{~V}=\mathrm{S}$

\begin{tabular}{|c|c|c|c|c|c|}
\hline & $\begin{array}{l}\text { P ARANCH } \\
\text { WAVE NLMBER } \\
(1 / \mathrm{CH})\end{array}$ & $\begin{array}{l}\text { UACLUM } \\
\text { WAUELENATH } \\
\text { CMJCRONSS }\end{array}$ & & $\begin{array}{l}\text { R BRANCH } \\
\text { WAUE NLMBEF } \\
\text { (I,CM) }\end{array}$ & $\begin{array}{l}\text { VACULH } \\
\text { WAVELENGTH } \\
\text { (MICKONS) }\end{array}$ \\
\hline $\begin{array}{l}\text { 1) } \\
\text { 2) } \\
3 \text { ) } \\
\text { 5) }\end{array}$ & $\begin{array}{l}3109 \cdot 14 \\
3073.95 \\
3037.56 \\
2999.93 \\
2961.09\end{array}$ & $\begin{array}{l}3.21643 \\
3.25315 \\
3.29211 \\
3.33341 \\
3.37713\end{array}$ & $\begin{array}{ll}\text { RC } & 3 \\
R C & 1 \\
R C & 2 y \\
R C & 3) \\
R C & 42 \\
R C & 5\end{array}$ & $\begin{array}{l}3175.15 \\
3296.99 \\
3235.55 \\
3263.56 \\
3289.90 \\
3314.70\end{array}$ & $\begin{array}{l}3.14945 \\
3.11906 \\
3.09066 \\
3.06419 \\
3.03961 \\
3.01686\end{array}$ \\
\hline $\begin{array}{l}63 \\
73 \\
83 \\
93 \\
63\end{array}$ & $\begin{array}{l}2921 \cdot 10 \\
2880.01 \\
2837 \cdot 86 \\
2794.70 \\
2750 \cdot 58\end{array}$ & $\begin{array}{l}3.42336 \\
3.47221 \\
3.52378 \\
3.57980 \\
3.63569\end{array}$ & $\begin{array}{l}R(6) \\
R C(7) \\
R(6) \\
R(9) \\
\text { RC } 10 ?\end{array}$ & $\begin{array}{l}3337 \cdot 87 \\
3359 \cdot 36 \\
3379 \cdot 16 \\
3397 \cdot 21 \\
3413 \cdot 49\end{array}$ & $\begin{array}{l}2.99593 \\
2.97675 \\
2.95932 \\
2.94359 \\
2.92955\end{array}$ \\
\hline $\begin{array}{l}13 \\
31 \\
31\end{array}$ & $\begin{array}{l}2705.55 \\
2659.65 \\
2612.94 \\
2565.46 \\
2517.26\end{array}$ & $\begin{array}{l}3.69611 \\
3.75989 \\
3.82711 \\
3.89794 \\
3.97258\end{array}$ & $\begin{array}{l}R(11) \\
R(12) \\
R(13) \\
R(14) \\
R(15)\end{array}$ & $\begin{array}{l}3427 \cdot 97 \\
3440 \cdot 61 \\
3451 \cdot 40 \\
3469 \cdot 29 \\
3467 \cdot 27\end{array}$ & $\begin{array}{l}2.91718 \\
2.90646 \\
2.89738 \\
2.88993 \\
2.88411\end{array}$ \\
\hline ? & $\begin{array}{l}2469.38 \\
2418.88 \\
2368.79 \\
2318.17 \\
2967.96\end{array}$ & $\begin{array}{l}4.05124 \\
4.13415 \\
4.22156 \\
4.31374 \\
4.41108\end{array}$ & $\begin{array}{l}\text { RSIG: } \\
\text { RCI } \\
\text { RCiG: } \\
\text { RCis } \\
\text { RC2D }\end{array}$ & $\begin{array}{l}3472 \cdot 32 \\
3475 \cdot 41 \\
3476 \cdot 50 \\
3475 \cdot 69 \\
3472.67\end{array}$ & $\begin{array}{l}2.87992 \\
2.87737 \\
2.87646 \\
2.87720 \\
2.87962\end{array}$ \\
\hline
\end{tabular}

\begin{tabular}{|c|c|c|c|c|c|}
\hline & $\begin{array}{l}\text { P ARANCH } \\
\text { WAVE NLMBER } \\
\text { (1/CH) }\end{array}$ & $\begin{array}{l}\text { WACLUM } \\
\text { WAVELENTTH } \\
\text { CMJCRONSS }\end{array}$ & & $\begin{array}{l}\text { R BRANCH } \\
\text { WAUE NLMBEF } \\
\text { (I,CM) }\end{array}$ & $\begin{array}{l}\text { VACULH } \\
\text { WAVELENGTH } \\
\text { (HICKONS) }\end{array}$ \\
\hline $\begin{array}{ll}P( & 1 \\
P( & 2 \\
P( & 32 \\
P( & 4) \\
P( & 5)\end{array}$ & $\begin{array}{l}3109 \cdot 174 \\
3073 \cdot 95 \\
3637.56 \\
2999.93 \\
2961.09\end{array}$ & $\begin{array}{l}3.21643 \\
3.25315 \\
3.29211 \\
3.33341 \\
3.37713\end{array}$ & $\begin{array}{ll}\mathrm{RC} & 0 \\
\mathrm{RC} & 1 \\
\mathrm{RC} & 2 \\
\mathrm{RC} & 3 \\
\mathrm{RC} & 4 \\
\mathrm{RC} & 5\end{array}$ & $\begin{array}{l}3175.15 \\
3296.09 \\
3235.55 \\
3263.58 \\
3289+90 \\
3314.70\end{array}$ & $\begin{array}{l}3.14945 \\
3.11906 \\
3.09066 \\
3.06419 \\
3.03961 \\
3.01686\end{array}$ \\
\hline $\begin{array}{l}668 \\
678 \\
682 \\
98 \\
102\end{array}$ & $\begin{array}{l}2921 \cdot 10 \\
2890.01 \\
2837.86 \\
2794.70 \\
2756.5 B\end{array}$ & $\begin{array}{l}3.42336 \\
3.47221 \\
3.52378 \\
3.57970 \\
3.63569\end{array}$ & $\begin{array}{ll}R( & 6) \\
R C & 7) \\
R( & B \\
R( & 9) \\
\text { RC } 10\end{array}$ & $\begin{array}{l}3337 \cdot 87 \\
3359 \cdot 36 \\
3379 \cdot 16 \\
3397 \cdot 21 \\
3413 \cdot 49\end{array}$ & $\begin{array}{l}2.99593 \\
2.97675 \\
2.95932 \\
2.94359 \\
2.92955\end{array}$ \\
\hline $\begin{array}{l}611 \\
1128 \\
1138 \\
1148 \\
6152\end{array}$ & $\begin{array}{l}2705.55 \\
2659.65 \\
2612.94 \\
2565.46 \\
2517.26\end{array}$ & $\begin{array}{l}3.69611 \\
3.75989 \\
3.82711 \\
3.89794 \\
3.97258\end{array}$ & $\begin{array}{l}R(11) \\
R(12) \\
R(13) \\
R(1) \\
R(15)\end{array}$ & $\begin{array}{l}3427 \cdot 97 \\
3440 \cdot 61 \\
3451 \cdot 40 \\
3469 \cdot 29 \\
3467 \cdot 27\end{array}$ & $\begin{array}{l}2.91718 \\
2.90646 \\
2.89738 \\
2.88993 \\
2.88411\end{array}$ \\
\hline$(16)$ & $\begin{array}{l}2468.38 \\
2418.88 \\
2368.79 \\
2318.17 \\
2267.96\end{array}$ & $\begin{array}{l}4.05124 \\
4.13415 \\
4.22154 \\
4.31374 \\
4.41108\end{array}$ & $\begin{array}{l}\text { R(16: } \\
\text { R(17) } \\
\text { R(16) } \\
\text { R(i9) } \\
\text { R(2D) }\end{array}$ & $\begin{array}{l}3472 \cdot 32 \\
3475 \cdot 49 \\
3476 \cdot 59 \\
3475 \cdot 69 \\
3472.67\end{array}$ & $\begin{array}{l}2.87992 \\
2.87737 \\
2.87646 \\
2.87720 \\
2.87962\end{array}$ \\
\hline
\end{tabular}


Appendix B - The Role of Numerical Stability in the Solution of Chemical Chain Reactions

This paper was presented at the 1971 Summer Computer Simulation Conference, Boston, Massachusetts, July 19-21, 1971, and appears in the proceedings. It attempts to illustrate in a simple minner the need for muterically stable schemes and the advantiages. 
Thic ta e preprint of a paper intended for publication in

- pearnel or proceedinis. Since changes may be made

UCRL - 73106

PREPRINT

bfert pulvetion, the preprint is mude available whth

the underelending thet it wall not be cited or reprodueed

withes the permieaion of the author.

\section{Jempexoo Fudiation Inboxetoxy \\ UWIVERSITY OF CALIFORMIA \\ IIVERMORE \\ 94550}

THE ROLE OF NUMERICAL STABILITY IN THE COMPUTER SOLUTION OF CHEMICAL CHAIN REACTIONS John Creighton

March 23, 1971

Prepared tor U.S. Atomic Energy Cómmission under contract no. W.7405-Eng-48

This paper was prepared for subraittal to the Proceedings of the 1971 Summer Computer Simulation Conference Boston, Massachusetts, July 19-21, 1971 
THE ROLE OF NUMERICAL STABILITY IN THE COMPUTER SOLUTION OF CHEMICAL CHAIN REACTIONS ${ }^{*}$

\author{
John Crelghton \\ Lawrence Radiation Laboratory, Univeralty of Calfornis \\ Livermore, California
}

\section{Summary}

Chemical chain reactions are reviewed briefly. Numerical stability is defined and illustrated, mainly in pictorial form, and an example shows how consideratlons of numerical stability limit the maximum step size in systems of "stif $f$ " differential equations such as chemical kinetic rate equations. Two methods that allow large time steps without introducing numerical instabilily are given.

\section{Introduction}

The need to calculate numerical solutions of chemical kinetic rate equations arose in trying to con truct a numerical model of a chemical laser. In a fast exothermic chemical reaction. the product molecules may exist for a short time in vibrationally excited states, which can be used, under certain conditions, to create an infrared laser. The first step in calculating the laser output is to solve the chemical kinetic rate equations to obtain the rate of formation of excited product molecules. In doing this we found that numerisal stability was crucial to obtaining efficient procedures. Discussions with other groups working an similar problems indicated that the need for numerical stability was common. A brief survey of the literature on detonation indicated that some attempts to solve kinetic rate equations had fountored an the rocks of numerical instability. It appeared, therefore, that our results would be applicable to a broad class of problems.

Although the phenomenon of numerical instability is well known to humerical analysts, $1-15$ it does not seem to be familiar to people trained in other disciplines. Therefore, this puogr is ai attempt to present the subject of numerical stability in a simple manner, witl. apologies to the expert who is likely to see little that is new. As an aid to understanding, the topic has been developed largely in plctorial terms. More rigorous derivations can be found in the standard references on numerical analysis and in the literature.1-15

The preaentation is divided into four sections: a briel revlew of chemical chain reactions; the definition and illustration of numerical stability: an exemple of the need for numerical stability. to obtain lerge tinıe steps; and suggested rechniques for obtaining the required stability.

\section{A Brief Revlew of Chemical Chain Reactions}

An exampie of a gas phase chemical chain reaction frequently found in textbooks is the: photorkemical reaction of hydrogen and bromine, 26 The uverall reaction is

$$
\mathrm{H}_{2}+\mathrm{Br}_{2}=2 \mathrm{HBr}
$$

If we break the reaction up finto ateps corresponding to chemically reactive collibions of molecules in a gas, the following steps turn out to be the dominant reactions:

$$
\begin{aligned}
& \mathrm{Br}_{2} \stackrel{\mathrm{k}_{1}}{=} \mathrm{Br} \text { Photochemicel } \\
& \text { chatn initiation } \\
& \left.\begin{array}{l}
\mathrm{Br}+\mathrm{H}_{2} \underset{\mathrm{k}_{-2}}{\mathrm{\textrm {k } _ { 2 }}} \mathrm{H}+\mathrm{HBr}+\mathrm{H} \mathrm{r}_{2} \stackrel{\mathrm{k}_{3}}{\longrightarrow} \mathrm{HBr}+\mathrm{Br}
\end{array}\right\} \begin{array}{l}
\text { Chain } \\
\text { reaction }
\end{array} \\
& \mathrm{Br}+\mathrm{Br}+\mathrm{M} \underset{\mathrm{k}_{-4}}{\stackrel{\mathrm{k}_{4}}{\rightleftharpoons}} \mathrm{Br} \mathrm{r}_{2}+\mathrm{M} \\
& \mathrm{Br}+\mathrm{urall} \stackrel{\mathrm{k}_{5}}{\longrightarrow} 1 / 2 \mathrm{Br}_{2} \text { (wall), }
\end{aligned}
$$

where I represents the density of absorbed photons ${ }^{a}$ and $M$ is any third body that does not react chemically in a three-body collision. The third body is necessary in recombination reactions, to conserve energy and momentum. The reaction rates (e.g., k2) defiend on the frequency of collision and the probabllity of reaction during a collision. The principle feature to be noted is the chain formed by Eqs, 3 and 4. Equation 2 produces a bromine atom (Br) that is captured in Reaction 3 but in the procesa produtes a hydrogen atom (H) that in being captured produces a new bromine atom. If Reactions 5 and $\mathrm{C}$ were not occurring, the reaction would loop continuously through Reactions 3 and 4 until the $\mathrm{H}_{2}$ or $\mathrm{Br}_{2}$ were used up. However, Reactions 5 and 6 provde alternative reactions for the bydrogen and bromine atoms, removing thern from the chaln. In those reactions charncterfzed as chain renctions, the rate of removal by Reactions 5 and 6 is usually relatively small, whth the result that for every bromine atom created by absorption of onc photon there may be $10^{2}$ to $10^{6} \mathrm{HBr}$ molecules formed before the hydrogen and bromine atoms are removed. The ra.io of $\mathrm{HBr}$ molecules to absorbed photons is ca]led the "chain length."

Work yerformed under the auspices of the $v$. S, Atomic Energy Commission, 
To calculate the concentrations as a function of tims, we roust obtain rate equations. Using the example above and Ietting $[\mathrm{X}]$ be the number of molpeules of type $X$ per unit volume, we can wite the following equations for the rate of change of number deasity (or concentrations, in an tdoul ano mixture):

$$
\begin{aligned}
& \frac{d\left[\mathrm{~B}_{2}\right]}{d l}=-k_{2}[\mathrm{Br}]\left[\mathrm{H}_{2}\right]+\mathrm{k}_{-2}[\mathrm{HBr}][\mathrm{H}] \\
& d\left[\mathrm{Br} r_{2}\right]=-\mathrm{k}_{1}\left[\mathrm{I}_{\mathrm{A}}\right]\left[\mathrm{Br} r_{2}\right]-k_{3}[\mathrm{H}]\left[\mathrm{Br}_{2}\right] \\
& \quad+k_{4}[\mathrm{Br}]^{2}[\mathrm{M}]-\mathrm{k}_{-4}\left[\mathrm{Br}_{2}[\mathrm{M}]+1 / 2 \mathrm{k}_{5}[\mathrm{Br}]\right.
\end{aligned}
$$

$\left.\frac{d[H]}{d t}=k_{2}[\mathrm{Br}]\left[\mathrm{H}_{2}\right]-k_{-2}[\mathrm{HBr}] j \mathrm{H}\right]$

$$
-\mathrm{r}_{3}[\mathrm{H}]\left[\mathrm{Br} \mathbf{B}_{2}\right]
$$

$$
\begin{aligned}
\frac{d[\mathrm{Br}]}{d t} & =2 k_{1}\left[\mathrm{I}_{\mathrm{n}}\right]\left[\mathrm{Br} \mathrm{Br}_{2}\right]-\mathrm{k}_{2}[\mathrm{Br}]\left[\mathrm{H}_{2}\right] \\
& +k_{-2}[\mathrm{HBr}][\mathrm{H}]+k_{3}[\mathrm{H}]\left[\mathrm{Br}_{2}\right] \\
& -2 \mathrm{k}_{4}[\mathrm{Br}]^{2}[\mathrm{M}]+2 \mathrm{k}_{-4}\left[\mathrm{Br} r_{2}\right][\mathrm{M}]-\mathrm{k}_{5}[\mathrm{Br}]
\end{aligned}
$$

$\frac{d f H B r}{d t}=k_{2}\left[B r \mid\left[H_{2}\right]-k_{-2}[\mathrm{HBr}][\mathrm{H}]\right.$

$$
+k_{3}\left[B r_{2}\right][H] \text {. }
$$

In these equations we have assumed that

$$
[\mathrm{M}] \cdot\left[\mathrm{H}_{2}\right]+[\mathrm{H}]+\left[\mathrm{Br}_{2}\right]+[\mathrm{Br}]+[\mathrm{HT3r}] \text {. }
$$

Thene ore coupled firat-order nonlinear differential equations with the number densities of the various species as variables. If the equations were dinear they could be solved by straightforward matrix mothods, 5 but since the terms contain products of two variables this cannot be done. (The often-used steady-stute approximaIlon linearizes the equations by holding some concentretions constant, meking the equations linear.) Since there is no general mothod for olving nonlinear differential equations, we must resort to numerical methods.

The obvious advantages of computer code to nolve there equations are that it is usually more economical than an experiment, and it can be uned for dealgn of herdware systems, It aiso reduces the necessity for handling material a that may be dangerous. An important advantage of the numericel solution that may not be oisvious is this: It can yield a qualitative undurstanding of the reaction. It may be very difficult experimentally to measure the concentration of some intermediate species $\mathrm{s}$ uch as [H], but the numerical solution gives this as a matter of course. Examination of a few computer printouts can rapidly indicate which features of the reaction are dominant.

In view of these advantas $=5$, computer solution of equations such as 6 through 11 ought to be commonplace, but there are some problems. There are often insurficient data on the chemical kinetic rates $\left(k_{\text {, }}\right.$, etc.). and the large effect of trace ir. 'Irities in experiments can cause large discrep.icies between calculated and experimentil results. However, 1 wish to concentrate on a third problem: numerical instabilixy.

\section{The Nature of Numerical Stability}

Numerical instability is the generation of erroneous solutions to differential equations because of deficiencies in the numerical scheme. The errors are frequently catastrophic in that they generate erroneous numbers approaching infintty. We must also be clear about what is not meant by numerical instability. In particular, it is not a matter of an instabiltty of the system being simulated; we ann assume the system to be stable and yet obtain trroitenus solutions due to numcrical itistobility. Also. numerical instability is net a programmíng "bug": a procedurc that is programmed completciy without bugs can have numerical instability. Numerical instability is not the roundoff error of the computer, or the trunce ion error of a series expansion, though it is related to these errors. I wish to illustrate this pictorially, give the conditions for numerical stability, and briefty discuss two methods that are numerically stable for large time steps.

Numerical instability is analogous to the instability of an amplifier with fecdback. In an unstable numerical method, the truncation and round-off ermes constitute "noise" that is amplified by the numerical scheme so that the erroneous output destroys the cor rect response. The existence of numerical instability depends on the numerical method and the time inerement that is used.

When we solve the differential equaticn $\mathrm{dy} / \mathrm{dt}=-y$, we get a family of solutions of the form $y=y_{0} e^{-t}$, with eacn curve in the family characterized by a different value of the parameter $y_{0}$, A rew of these curves are shown in Fig. I. Since $y_{0}$ is a continuous variable there is, of course, an infinite number of internicdiate curves lying between those shown in Fig. 1. When we set $y_{0}$ equal to some 1ritial value we select one curve out of the family of soluticus as our solution - as we selected the curve for $y_{0}=0.5$, shown in Fig. 1 . A numerical solution is shown for comparison.

Sote, fowever, the racent exlenstve tablas of kinetic rate thta in Kefs. 17 through 19, and the methods for estimating rate constants aiven by Benson. 20 


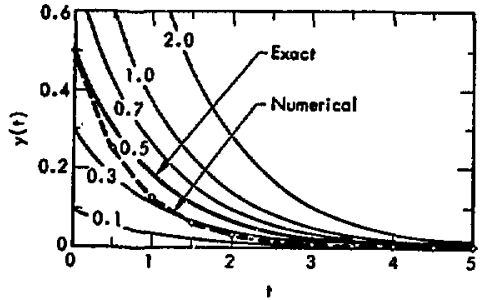

Fig. 1. The solutions of the equations $d y / d t=-y$, for several values of $y_{0}$. The solution for $y_{0}=0.5$ is shown with the corresponding numerical solution for $\Delta t=0.5$.

The simple method used for this solution consists of calculating the derivative at a point $y\left(t_{k}\right)$ and drawing a short line at that slope over a time step $\Delta t$ to the point $y\left(t_{k}+\Delta t\right)$, then repeating the process. That is,

$$
y\left(t_{k}+\Delta t\right)=y\left(t_{k}\right)+\Delta t\left[d y\left(t_{k}\right) ; d t\right] .
$$

Note that in the first step the new point lies on a curve (not shown) that is not the exact solution for $y_{0}=0.5$ but that is the solution for a slightly amalier value of yo. At each point the new $y\left(t_{k}\right)$ lits on a different solution curve from the pre vious one; but the error in the numeriral solution remains small because each new point gives a value of the derivative, which forces the numertcal solution back toward the true solution.

Contrast this with the situation in Fig, 2 , which shows the solutions, $y=y_{0} e^{t}$. of the differential equation $d y$ idt $=y$. Once again each step from $y\left(t_{k}\right)$ to $y\left(t_{k}+\Delta t\right)$ moves the numerical solution from one curve to another on the family of analytic solutions. Now, however, the slope on the new curve forces the numerical solution further from the true solution, and in a few cycles the numerical solution will be nowhere near the true solution. This is numerical inatability. These illustrations can be generallzed to a rule for numerical stability: when the family of analytic solution curves in converging toward some single curve, we have numerical stability: If the family of curves is not converging, wo do not heve numerical stability. This can be stated more sccurstely and concisely in algebratc fors. wo have numerical stability when

$$
\theta y^{\prime} / 0 y=0,
$$

where:

$$
y^{\prime}=d y / d t \text {. }
$$

in the reglon where we scek the solution; otherwlse, we do nat have numerical stabtllty. Note that this condition is precisely what we have in

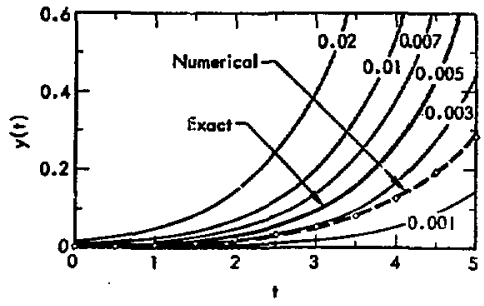

Fig. 2. The solutions of the equation $d y / d t=y$, for several values of $y_{0}$. The solution for $y_{0}=0.005$ is shown with the corresponding numerical solution for $\Delta t=0.5$.

Fig. 1, where we slowed geometrically that we had numerical stability. This criterinn is valid for the present method and other popular ones. Wo shall see later that the criterion may be that $\partial y^{\prime} / \partial y$ is less than some constant $D$.

In the case where $\partial y^{\prime} / \partial y>0$, as in Fig. 2, all is not lost. If we keep the time step very small, we can keep our numerical solution on curves that lie very close to the exact solution, and the numerical error will be small even after tnany time steps, In particular, if $\Delta y(t)$ is the difference between the numerical and true solutions at time $t$ we say that we have "relative stability ${ }^{h}$ if the ratio $\Delta y(t) / y(t)<c$ over the entirs range of $t$ where we seet: a solution, and $c$ is some constant. We usually wish to keep c small compared with $y(t)$; or, more colloquially. we have "relative stability" if we keep our percentage error small.

One other type of in:stability is relevant to the problem of chemical chain reactions.

in Fig. 3 we return to the equation of Fig. 1 . which was slown to be numerically stable because the family of solutions converged toward zero. Assume that we are interested only in the solution for $t \gg 1$. so we plot the solution on a different scale, and to save computer time we take a larger time step. The numerical solution. using the same method as Fig. 1 with $\Delta t$ increased to 2,2 , is shown for comparison. The solution oscillates with growing amplitude and in a few more otepa would "blow up" completely.

This osclllation occurs because we have approximated the exact solution by a Taylor series, which for the method we used is terminated after the second term; that is,

$$
y(t+\Delta t)=y(t)+\Delta t[d y(t) / d t]+\ldots
$$

In this example we have taken $\Delta t$ ao large that the error of order $(\Delta t)^{2}$ is large compared to the solution, resulting in osclliations, Some authors call inis "partial instability."i

The numerical method we have chosen $1 \mathrm{a}$ a poor one for artual calculations, but it Illustrates 


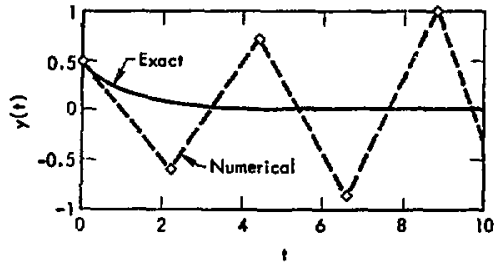

Fig. 3. An example of "partial instability" due to an excessively large time step. for the equation $\mathrm{dy} / \mathrm{dt}=-y$. The exact solution for $y_{0}=0.5$ is shown with the corresponding numerical solution for $3 t=2.2$.

in simple form behavior that is common to most mothods. References on numerical analysis $1-7$ and the ltteratur $c^{8-15}$ give a number of methods for numerical solution of differential equations. Most authors preier multipoint methods and implicit methods. Implicit methods use infornation about the new point, to oltain the value of the new point, This often rcsults in algebraic equations that must be solved by iterative techniques, For example, we could average the slopes at $t_{k}$ and $t_{k}+\Delta t$ as follows:

$$
\begin{aligned}
y\left(t_{k}+\Delta t\right)= & y\left(t_{k}\right\}+1 / 2 \Delta t \\
& \left.x \mid y^{\prime}\left(t_{k}\right)+y^{\prime}\left(t_{k}+\Delta t\right)\right\} .
\end{aligned}
$$

This must be solved iteratively. We can use our former method to obtain a trial valie of $y\left(t_{k}+\Delta t^{3}\right.$. This is called tive "predictor" stcp and usuaily requires an explicit formula. This new trial value is substituted In the right hand side of Eq. 16, and the process is repeated until two auccessive trial values agree within aome small error limtt. This is the "eorrector" phase of the calculation. This is one of the aimplest implicit mothods. A simple multipoint formula is

$$
\begin{aligned}
y\left(t_{k}+\Delta t\right)= & y\left(t_{k}\right\rangle+1 / 2 \Delta t \\
& x\left\{3 y^{\prime}\left(t_{k}\right)-y^{\prime}\left(t_{k}-\Delta t\right)\right\} .
\end{aligned}
$$

In the multipoint method, previously calculated values of the derivative are used to obtain either - better predlction of the new point or a smaller truncation crror in the corrector. Equntion 17 approximates tho derivative by a parabola. whereas the method used in the previous exan:ples approximaied it by a straight line, In noost 1 ases, the parabola will give a more accurate prediction.

The advantages of these netliods are grodier sccuracy ard stabilfty, which pernit larger time steps. However, all these mothods are characterized by a certain domain of stability,2,3,8,9

The domain of staljility for several popular methods is shown in Jig. 4, The figure represents the complex $\$\left\{\left(\partial y^{\prime} / \partial y\right)\right.$ plane that corresponds to solutions of the form $\mathrm{e}^{(x+1 y) t}$. The factor $e^{\text {iyt }}$ represents oscillatory solutions; $e^{x t}$ is the damping factor if $x$ is negattve, and the amplification $j$. ctor if $x$ is positive.

In many references $\Delta t$ is rcpresented as $h$, dy $/ d t$ is represented as $I(x, y)$. and stability is expressed in terms of the parametcr $h(\partial f: \partial y)$. If we have a system of several differential equations in variable $y k$, stability, is determined by the Jacobian matrix $\left(\partial y^{\prime} k^{i} y_{j}\right)$. If we call its efgenvalues $\lambda_{j}$, we must havech $\lambda_{i}$ lying in the stabitity domain for all the eigenvaiucs $\lambda_{i}$.

The Relationship hotween Tinje Step Size and sunericnl stibility

When one atterapts to solte chemical kinetic rate equations, he finds that he generally has a syster. of "siffe nquations." Thits simply meal:s that the solution lias several time constants. some of which are orders of mamitude faster than others. This rauses atability proviems tiat can best be illu: trates uy an exampie.

The motion of a clamped harmonic oscillator can be siescribct by the pair of equations:

$$
\begin{aligned}
& (\mathrm{dy}, \mathrm{d} u-v=0 \\
& (\mathrm{d} v, d \mathrm{dt})+\gamma v+\sim^{2} \mathrm{y}=0,
\end{aligned}
$$

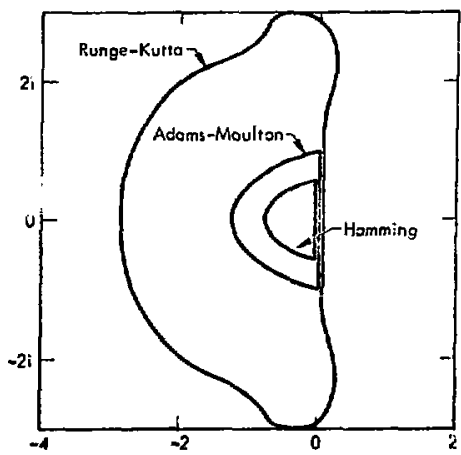

Flg. 4. Domain of stability for three popular numerical schemes. The donain is plotted on the complex $\Delta t(\partial y / \partial y)$ plane. witl the imaginar part represontine oscillatory solutions rind llie weal part representing mponentislly recuying or frowing fncto:s. Isased on rata provided in liei. 3 . 
where $y$ can represent displacement, and $v$ the velocity, For simplicity let $\gamma=10,1$ and $\omega=1$, an overdamped case, If we let $y(0)=0$ and $v(0)=9.9$, the solutions are (to a good approximation)

$$
\begin{aligned}
& y=-e^{-10 t}+e^{-0.1 t} \\
& v=10 e^{-1 c t}-0.1 e^{-0.1 t} .
\end{aligned}
$$

This is shown in Fig. 5.

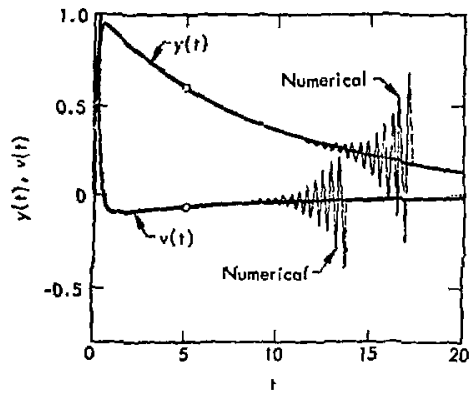

Fig. 5. The solution of the overdamped harmonic oscillator equations $d y d t=\psi$ and $d v / a t=-y-10.1 v$, with initial conditions $y(0)=0, v(0)=0.9$. Exact solutions are shown along with numerical solutlons, using exact values at $t=5$ for starting values, The time step, $\Delta t=0,22$, was large enough to cause the oscillations shoirn.

The solutions show that, for $t>1$, onjy the second term in each solution is significant, bocause the finst term has died doun. Therefore, it would seem natural in loing a numerical solution for times $t>1$ ta use a time step appropriate to the stowly varying $\mathrm{term}, 1$,e. $\Delta t \sim$ in, it turns out, lowever, that wo are forced to use a time step such that $\Delta t \sim 0.1$. To illustraze this paint, a nimmerical * ' utian of Eqs. 18 and 10 is shown in Flo. So. The sime stej, $\Delta t$, was 0.22 ; for the fast term, - $e^{-10 t}$, this time step curresponds to the $\Delta t=2,2$ in Figs. 3 . The numertcal solution was started at $t=5$, using the analytic solution for itarting values. The growth of the instability is clear from the figure. This illustrales the point that the size of the time step is controlled by the tistest terms in the solution. If we had chosen $y=1000$ is 2 in Fiqs. 18 and 17 , it would take on the urcier of a millinn time steps to reach the pount ificge bio sursiv varying turm was one-tont, , tximum, On ail but the fastest computers ti.is mignt take an hour.
Two Methods tor Obtining large Time Stcos:

There are two ways to get around this probler.: and obtain rapid numerical solutions of systems of "stiff equations."

One method is to build into the computer program logic that notes the faet thut some terms in the solution are no longer important and then modifies or eliminates certain equations from the systern. In our eximple this would be done by setting $d v / d t$ to zcro in Eq. 19, solving the equation for $y$, and substituting the value in Eq. 18 to give

$$
\mathrm{d} y / d t=-\omega^{2} y / t=-0,1 y,
$$

The solution of this equation is the sccond term of $\mathrm{Eg} .20$, so we have a stable method that can use time steps with at $\sim 10$ and with negligiblc error for $t>1$. The stability for $\Delta t=10$ and is was verified by a nimerical calculation. it is not a trivial task to program this for large systems of equations, but it has been done successiully by G. Emanucl in the NES'P chemical kinetics code. 21,22

Another approach is to une a method that includes larpe negative valucs of $\Delta t$ tay'joyt in its domain of stability. In this case the rapidly decaying terras in the solution do $\mathrm{nr}$. cause instability. and lares time stens can b: taken uthec these transients hive clied out. $c$. W. Goar has Written a lOT $7 \mathrm{~T} A \mathrm{~N}$ subroutine that does just
this. $12-14$

The stability domain of the Gear method is shown on the complex hi, plane in Fitf. 6 . Remembering that $h$ is the time step and $\lambda$ the elgenvalues of $\left(b y_{k}, y_{j}\right)$, we see that the methorl is stable ishown by the shaded rection' onity ior values to the ieft of the vertical tinc lic(t) $\lambda)=-D$. but extends to minus intinity. Comparing this with Fig. 4, we see that stability in the ragion -1) Re(hx) $\leq 0$ is sacrificed to exinnd the slomain to minus infinity. Within the sniall, cross hatched rectangle there is oniy "relative stability", which means that the error must $s$ e kejh small in this rogion. The crror win be small if we kcep the quantity id sinall.

In the Gear techique. the parameters determining the size of the rectangle- $D, 0$, and $a-$ are chosen so that any valve of $h \lambda$ lying in the rectangle will give: an accurate solution. The time step h can now be chosen so that hx for the slowly vatying terms in the solution lie in the rectangle, but those correspondits to y isidy deeaying terms lie in the region of absolki* stability and to not limit the size of the time stcp. This was precisely our goal to have the time step controlled by the slowly varying certis. The subroutine has qutomatic error and t*me : $^{*} \mathrm{ep}$ controls that are interconnected to give th.". largost time step that will keep the erro within acceptable limits.

We tave used this suldroutine to solve thrs $\mathrm{II}_{2}+$ Bra reaction (lias, 2-6), which has timo cunstinnts

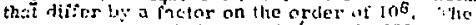

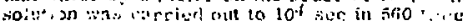
strpis. wireh requirea iess than a trinte a: am.

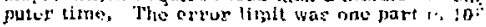




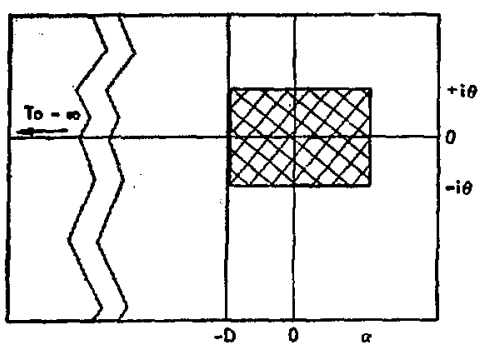

Fig. 6. Domatn of stabllity for C.W. Gear's method plotted on the complex $h \lambda$ plane. The crosshatched rectang]e indicates the area of relative stability. The shaded area is the rtgion of absolute stability.

per step. Flgure 7 is plot of log $\Delta t$ vs log $t$, which shows how the time step increases as the ransients die out. This subroutine is available $\boldsymbol{x}$ general use.

\section{Conclusion}

In conclusion, let us review brictly the importance of numerical stability to the numerical solution of chemical chain reactions. The rate equations are "stiff" in the sense that they have characterlatic time $в$ that vary by several orders of magnitude. The shortest of these times will control the size of the maximun time step uriess epeclal methods are used. One mothod is to force certain derivatives to be zero (the steadystate approximation), and another is to use numerical techniques that are stable cven when theso fast terms are included.

\section{Acknowledgments}

I would like to thank Drs, R. Gellnas and A. Hindmarsh for bringing $C$. W. Gear's routine to my attention and for implementing it at our laboratory. Thoy have also been of assiatance in underatending the nature of numerical gtability. I also am indebted to $C$. W. Gear, whose subroutine has made my work so much caster.

\section{Appendix}

\section{Rapld Computer Evaluation of Hate Equation}

in a practical problem there may be as many an 100 equations of the form of EqB. $(2)-(6)$, involving 20 molecujar apecies. Fortunately, in the interests of accuracy and our sanisy, a simple computer algorithin can derive the rate equations of the form of Eqs. (7)-(1:).

\footnotetext{
There are two sources for this ubbroutine, 23,24
}

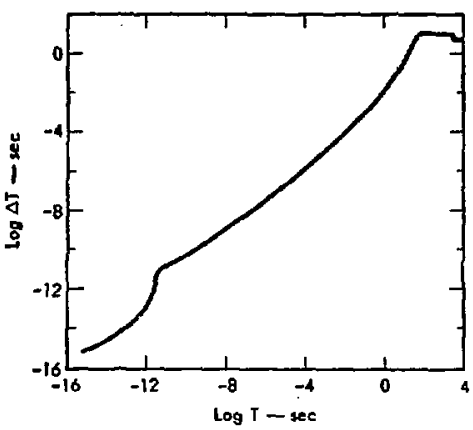

FIg. 7, A log-log plot of time step vs time for the $\mathrm{H}_{2}+\mathrm{Br}_{2}$ reaction, ustng the Gear numerical schome. The upper limit is arbitrarily set at 12 sec.

.et the concentration of each molecular species $u c$ a component $y_{i}$ of a column vector $\bar{y}$, and let the stoichiometric number for the $i^{\text {th }}$ spectes on the left-hend side of the ith reaction be the matrix element $r_{i j}$ of the matrix $k$. if we form a similar matrix $P$ for the stoichiometric number of the products on the riglit-hand side. we can write equations similar to Eqs. (2) in matrix form as

$$
(R) \bar{y} \leftrightarrow(P) \bar{y}
$$

For example, Eqs. (2)-(6) would be written

$$
\left(\begin{array}{lllllll}
0 & 1 & 0 & 0 & 0 & 0 & \Lambda \\
1 & 0 & 0 & 1 & 0 & 0 & 0 \\
0 & 1 & 1 & 0 & 0 & 0 & 0 \\
0 & 0 & 0 & 2 & 0 & 1 & 0 \\
0 & 0 & 0 & 1 & 0 & 0 & 0
\end{array}\right) \cdot\left(\begin{array}{l}
\mathrm{H}_{2} \\
\mathrm{Br}_{2} \\
\mathrm{H} \\
\mathrm{Br} \\
\mathrm{HBr} \\
\mathrm{H} \\
\mathrm{L}
\end{array}\right)=
$$

$$
\left(\begin{array}{lllllll}
0 & 0 & 0 & 2 & 0 & 0 & 0 \\
0 & 0 & 1 & 0 & 1 & 0 & 0 \\
0 & 0 & 0 & 1 & 1 & 0 & 0 \\
0 & 1 & 0 & 0 & 0 & 1 & 0 \\
0 & 1 / 2 & 0 & 0 & 0 & 0 & 0
\end{array}\right) \cdot\left(\begin{array}{l}
\mathrm{H}_{2} \\
\mathrm{Br}_{2} \\
\mathrm{H} \\
\mathrm{Br} \\
\mathrm{HBr} \\
\mathrm{M} \\
\mathrm{Ia}
\end{array}\right)
$$


If there are 1 molecular species and I reections, the matrices will have I columns and J rows.

Now the computer goes through the matrlces row by row and for the $\mathrm{j}^{\text {th }}$ row obtains a term

$$
T_{j}=k_{j} \prod_{i=1}^{1} z_{i}^{r_{i j}}-k_{-j} \prod_{i=1}^{I} y_{i j}^{p_{i j}} \text {, }
$$

where $k_{j}$ and $k-j$ are the forward and reverse rate constants. Note that these terms are the same as the individual Lerms on the right-hand side of the rate Eqs. (7)-(12).

The rate equation for the ith molecular specles is now obtained from

$$
d y_{i} / d t=\sum_{j=1}^{J}\left(r_{i j} T_{j}-p_{i j} T_{j}\right) \text {, }
$$

which glves a total of I rate equations with I unknowns. These rate equations are evaluated numerically at each time step. If a matrix element is zero, no operation is required except a test for zero and incrementing the index to test the next matrix element. This type of evaluation of dorivatives is therefore nearly as fast as evaluating the rate equations, yet it greatly reduces the chance of human error.

If modificatlons are made to the algorithm to manipulate labels of the molecular species Instead of numerical values, the computer can be made to print out the derived rate equations.

To read in the reactions in this form, it is necessary to punch the matrix elements on cards. It is possible to write a subroutine of a lew hundred FOR TRAN statenients that will read reactlons punched in approximately the format of Eqg. $(2)-(6)$ and canvert these data into the appropriate matrix form.

\section{Bibliography}

1. McCracken, D.D., and W.S. Dorn, "Numerical Methods and Fortran Programming with Applicetions in Englneering and Seience," Wifiey, New York (1964).

2. Hildebrand, F. B. "Introduction to Numerical Analysta," MeGrew-Hil, New York (1956).

3. Iinmming, A. W., "Numerical Methoda for Scientiate and Engineors," MCGraw-IHill. New York (1962).

4. Kelly, L. G., "Handbook of Numerical Method and Applicatlons," AddisonWesley, Rending, Matrachusetts (1867).

5. Ritger, P.D., and $N$. J. Rose." Differential Equatione with Applications, "MaGrewHIIl, New York (1968).
6. Raleton, A., and H. S. Wilf, "Mathematical Methoda for Digital Computers," Wiley. New York (1960).

7. Milne, W. E. "Numerical Solution of Differential Equations," Wiley, New York (1953).

B. Rahme, H.S., J. ACM, 16, 496-506 (1969).

9. Rahme, H.S. J. ACM 17, 284-293 (1970).

10. Hamming, R, W. T, ACM, 6, 37-47 (1958).

11. Nordsieck, A., Math. Conp., 16, 22-49 (1962).

12. Gear, C.W., Math. Comp, 21, 146-156 (1967).

13. Gear, C.W., Proc, JFIPS Conf, 1968, Edinburgh, pp. A8I-g5.

14. Gear, C.W., Digest Hecord, 1969 Joint Conf. on Math. and Comp. Aids to Design. Anaheim. October 27-3i. 1969. pp. 27-58.

15. Dahlquist, G., Math. Scand., 4, 33-50 (1956).

16. Benson, S, W, "The Foundations of Chemical Kinetics." pp. 319-331, McGraw-Hi]l. New York (1,60).

17. Benson, S. W., and H. E. O'Neal, "Kinetic Data on Gas Phase Cnimolecular Reacthons," NSRDS-NBS-21, U,S. National Bureau of Standards, V'ashington $(1970)$.

18. Trotman-Dickenson, A.F., and G.S. Milne, "Tnbles of Bimolecular Gas Reactions," NSRDS-NBS-0, U.\$. National Bureau of Standards, Washington (1967).

10. Baulch, D. L. D. D. IJrysdale, and A.C. Lloyd, Ifieh Temperature Reactions Rate Data Numbers 1-5, Dept, of Physical Chemistry, University of Lceds 2. England (1)G B-1970): address inquiries to D. L. Baulch.

20. Benson, S.W., "Thermochemical Kinetics," whley. New York (196U).

21. Emanuel, G. "Numerical Analysis of Stiff Equations," Hept. TDR-269 $(4230-20)-3$, The Acrospace Corporation, Fil Segundo, Callfornia (1964).

22. Turner, E. B, , G. Emanuel, and R. L. Wilkins, "The Nest Chemistry Computer Program: Vol, $i, " R e p t . T R-D 05916240-$ 20)-1. V1, The Acrospace Corporation. El Segundo, Californla (1970); also listed is Air Force Rept. SAMSO-TR-70-311, Vol. I (1970).

23. Hindmarsh, A., and R. Gelinas, "GEAR," to be published as CIC Rept. U2.?-003 by Lawrence Radlation Laboratory, Livermore. A FORTRAN deck for GEAA had also been prepared.

24. Genr, C.W. "Algorlthm 407," to he published In Commun. ACM, 14, 185(March 1971). 


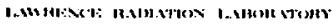
Hoxpr:

HIt.1M:-: 1:81:

M Е H R A D U H - July 27,1971

TO: Distribution

FHOM: J. Creighton

This memo is a technical atdendum to UCRI 73106, "The Role or Mamericel Stabjlity in the Computer Solution of Chemical Chain Reactions." You may vish to flle this with the original report.

Professor A. K. Oppenheirn suggested that I use the oscillator problen of eqs 18-21 as an 11]ustration of the power of Gear's method to solve stirf equations. The results are shown in the upper half of Figure $A$. For this problem the Gear method required 200 time steps to solve the system to the point where $y$ was less than .001 . It took 1 i 60 time steps to achtere the same result using the Adam's Predictor-Corrector option in the Gear subroutine, with automat1c tine step control. Both methods were started with a very smali time 5 tep whlch [rew roughly exponentially until the time oorresponding to the peak valie of $\mathrm{y}$. This required 150 time steps, after that the time step for the Gear method contiriucd to grow exponential lyk while the lime step for the Adsin's method stayed nearly constant. A detalled examination showed that under the Adan's method the time step increased until excessive error developed; at that point the time step was cut an order of magnitude, and vould egain increase until excessl error developed. However, when the step size wes averaged over tens of steps $1 t$ remalned constant for 1000 steps.

To obtain a more dramatic example I increased the damping factor $r$ to 100 so that the equivalent of equation 19 was

$$
\begin{aligned}
& d v / d t+130 v+y=0 \\
& \text { vith the solution } \\
& y=e^{-.01 t}=e^{-100 . t}
\end{aligned}
$$

As shown in the lower half of Figure $A$, the Gear method conpleted the proilem ir. 255 time steps. The Adan's method was terminated after 5000 time steps

* This behavior in 1llugtrated in F1g. 7 of the orlgtal papar (UCri 73106 ). 


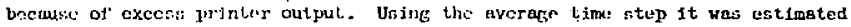
that it would inke over jod, non tjne ritrpa to eomplete tbo problem.

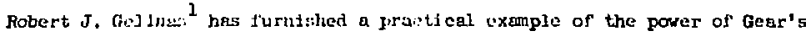
mothod in p photo-cturical smog catculation. Inis is showi in Figure B. Thre Adan's method was teminated for budgetary roasons. If the tias step were to remain constant for the rest of the problen it would requice millions or minutes of computer tims to ruach completion. Since nobody has thet sort of computer time it is elear that the problem requires a method that can handle stiff systems of equations. I belleve that these examples. show the power of Gear's mothod and the seriousness of the problen. of stifeness.

1 Robert J. Gelinas, "Stifr Systems of Kinetia Equations -- A Practitioner's View", University of Californde UCRL 73078 (to be published in J. Comp. Fhys) 1971 . 
THE GEAR METHOD RUNS MUCH FASTER THAN THE ADAM'S PREDICTOR-CORRECTOR.

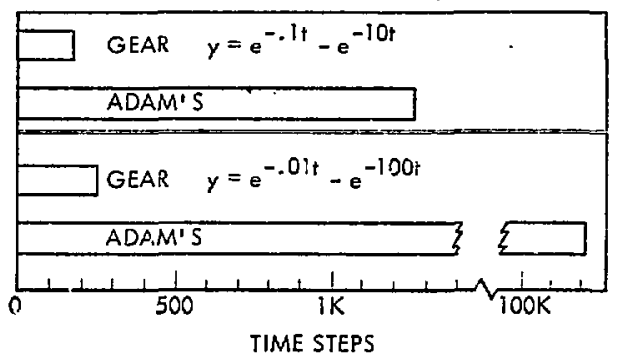

\section{FIGURE A}

A PHOTOCHEMICAL SMOG CALCULATION RUNS FASTER BY' GEAR'S METHOD ON CDC 7600

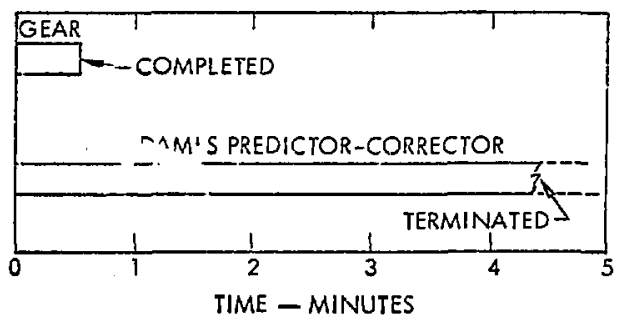

FIGURE B 
Appendix C - Commutators For the Hami teonianl of tquat ion 6.1 In order to solve the Heisenberz equations of morion, $E_{1} \cdot 6.2$, it is necessary to evaluace commutators for the Hamiltonian given in Eq. 6.1. These commutators have not been proviously evaluated for the 3-level system of Section 6. The conmutators were calculated from the matrix representations of Section 6.1 , and are given below.

I. Radiation Creation and Annihilation Operators The operators $\mathrm{a}^{+}$and a commute with all the molecular operators, $\sigma$ and $n$. The operators $a_{1}^{+}$and $a_{1}$ commute with $a_{2}^{+}, a_{2}, a_{3}^{+}$, and $a_{3}$ because they span a different space. The non-vanishing commutators are

$$
\left[a_{k}, a_{k}^{+}\right]=1=-\left[a_{k}^{+}, a_{k}\right] .
$$

Other useful commutators are

$$
\left[a_{k}, a_{k}^{+} a_{k}\right]=a_{k},\left[a_{k}^{+}, a_{k}^{+} a_{k}\right]=-a_{k}^{+} \text {. }
$$

The subscript $k$ refers to one transition, but the commutators vanish if $k$ is not the same for all oscillators.

11. Molecular Raising and Lowering Operators

The raising operators $\sigma_{1}^{+}$and $\sigma_{2}^{+}$occur in the Hamiltonian (Eq. 6.1) as do the lowering operators. In evaluating commutators we obtain a new lowering operator

$$
\sigma_{3}^{-} \equiv\left[\sigma_{2}^{-}, \sigma_{1}^{-}\right] \text {. }
$$

We also obtain a raising operator

$$
\sigma_{3}^{+} \equiv\left[\sigma_{2}^{+}, \sigma_{1}^{+}\right] \text {. }
$$

These two operators would appear in the Hamiltonian if $b_{3}$ were not zero. 
The raising and lowering operators comnute with radiation operialors. In penreral $[A, B]=-[B, A]$, which imp]ies $[A, A ! \cdots 0$. Commutation of raisinf and lower operators with popidation operators will be considered iri the next section.

The commutators of raising and lowering operators with others are given below

$$
\begin{aligned}
& {\left[\sigma_{1}^{-}, \sigma_{2}^{-}\right]=\sigma_{3}^{-}=-\left[\sigma_{2}^{-}, \sigma_{1}^{-}\right]} \\
& {\left[\sigma_{1}^{-}, \sigma_{3}^{-}\right]=0=-\left[\sigma_{3}^{-}, \sigma_{1}^{-}\right]} \\
& {\left[\sigma_{2}^{-}, \sigma_{3}^{-}\right]=0} \\
& {\left[\sigma_{1}^{-}, \sigma_{1}^{+}\right]=n_{1}, n_{2}} \\
& {\left[\sigma_{1}^{-}, \sigma_{2}^{+}\right]=0,\left[\sigma_{1}^{-}, \sigma_{3}^{+}\right]=\sigma_{2}^{+}} \\
& {\left[\sigma_{2}^{-}, \sigma_{1}^{+}\right]=0} \\
& {\left[\sigma_{2}^{-}, \sigma_{2}^{+}\right]=n_{2}-n_{3},\left[\sigma_{2}^{-}, \sigma_{3}^{+}\right]=a_{1}^{+}} \\
& {\left[\sigma_{3}^{-}, \sigma_{1}^{+}\right]=-\sigma_{2}^{-}} \\
& {\left[\sigma_{3}^{-}, \sigma_{2}^{+}\right]=\sigma_{1}^{-},\left[\sigma_{3}^{-}, \sigma_{3}^{+}\right]=n_{1}-n_{3}} \\
& {\left[\sigma_{1}^{+}, c_{2}^{+}\right]=-\sigma_{3}^{+}}
\end{aligned}
$$

III. Population Operators

The population operators $n_{1}, n_{2}$, and $n_{3}$ commute with i11 radiation operators and with each other; they do not commute with all of the raising and lowering operators. 
282

Those commutators are

$$
\begin{aligned}
& {\left[\mathrm{n}_{1}, \sigma_{1}^{-}\right]=\sigma_{1}^{-}} \\
& {\left[\mathrm{n}_{1}, \sigma_{2}^{-}\right]=0} \\
& {\left[\mathrm{n}_{1}, \sigma_{3}^{-}\right]=\sigma_{3}^{-}} \\
& {\left[\mathrm{n}_{1}, \sigma_{1}^{+}\right]=-\sigma_{1}^{+}} \\
& {\left[\mathrm{n}_{1}, \sigma_{2}^{+}\right]=0,\left[\mathrm{n}_{1}, \sigma_{3}^{+}\right]=-\sigma_{3}^{+}} \\
& {\left[\mathrm{n}_{2}, \sigma_{1}^{-}\right]=-\sigma_{1}^{-}} \\
& {\left[\mathrm{n}_{2}, \sigma_{2}^{-}\right]=\sigma_{2}^{-}} \\
& {\left[\mathrm{n}_{2}, \sigma_{3}^{-}\right]=0} \\
& \left.\left[\mathrm{n}_{3}, \sigma_{2}^{+}\right]=\sigma_{2}^{+},\left[\mathrm{n}_{3}, \sigma_{3}^{+}\right]=\sigma_{3}^{+}\right]=\sigma_{1}^{+} \\
& \left.\left[\mathrm{n}_{3}, \sigma_{2}^{+}\right]=-\sigma_{2}^{+},\left[\mathrm{n}_{2}, \sigma_{2}^{+}=\sigma_{1}^{-}\right]=\sigma_{3}^{-}\right]=-\sigma_{2}^{-} \\
& {\left[n_{3}\right]}
\end{aligned}
$$

The physical significance of these commutators is clear. For example, the commutator $\left[n_{j}, \sigma_{k}^{\ddagger}\right]$ represents a raising or lowering operator on transition $k$ interacting with level $j$. I1 the interaction increases the value of $n_{j}$, then 
$\left\lfloor n_{j}, \sigma_{k}^{ \pm}\right]=-\sigma_{k}^{ \pm}$. If there is no change in $n_{j}$ the commutator is zero. The commutator of the raising and lowering operators for a single transition is the inversion, and the commutator of two lowering operators $\left[\sigma_{1}^{-}, \sigma_{2}\right]$ is $\sigma_{3}^{-}$ which is an equivalent operation. If we had understood the physical significance beforehand we could have written the commutators by inspection, and we can now use our physical insight to check our algebra. 
Appendiv :) - A Simple Analytic Nodrs for Rotat ional

Relaxal.ion

hinen this work bepall thore was one set of mperimemal

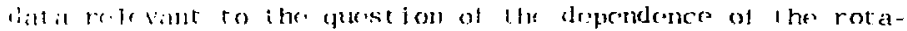
tional rolixation rats on rotalismal flantlun number in $\mathrm{HF}$.

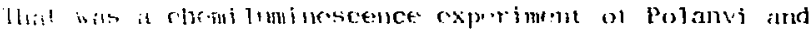
bored:111 (1972a). They reacted f wilh H2 in at flowing systent alli observed the popilations at sevwral dewnstren stations by mons of chemi inminescences. Starting with the initial distrinution thes caleulatorl thr townst rean distribut ions

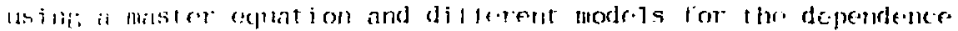

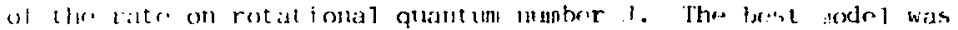

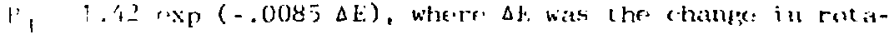

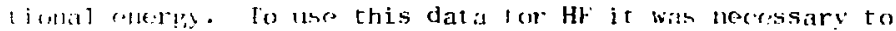
know what physical parameters were inportant in catsing,

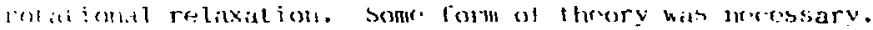
Ihe thesorelicil literature on rotational relaxation i - oriciam rowards ultrasonics and other applications

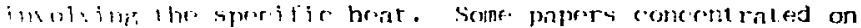

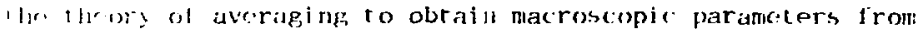
'sitroks opic models (Brau \& Jonknan, 1970; de Boer $\varepsilon$ lthlenbeck, 196, Palf $\varepsilon$ linter, 1968). Another group ol papers utied turdels iuch as rough sphere, and prolate and oblate spheriods (sather \& hah]er, 1961 \& 1962; hidom, 1960). Jhess "merhanical" moxdele dm't sem vory realistic, oven for mompolar molecules.

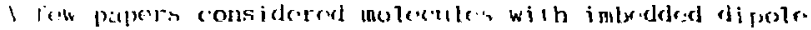
moments or diattimic molecules with lores betwecen all four 
atoms (Brout, 1954; Cross \& liordon, 1966; Nyes land, 1967; Parker, 1959; Zelowilk, 1967, 1970, 1971). These: siemed a beat ter modf.1 for HF, lilt thry all assuned no inital rotation, so were liseless for calculat ing rotat ional reprindenct:

Ir seened appropriatr, therefore, to calculate rotational relaxation based on a simpl" model using imbedded dipeles in hard spheres with initial rotation. Belore the. modrl was completed 1 observed that 7 inewidths in $\mathrm{HF}$ (Meredith, 1972) varied with rotacional quantum number and the variation was quite similar to the rotational relaxarion in HC:1 observed by Polanyi and hooda11 (1972a). The Anderson theory of line broadenine (Anderson, 1949; Tsao \& Curnutte, 1962) and similar theories (Murphy \& Boges, 1967 to 1969) aro in ract theories of rotational relaxation assuming multipole interactions briween noleculus. lihn rotational frequencies are in the microwave region, the relaxation is entirely dur to rotational relaxation. In the visible a large traction of the 1 inewidth $j$ s dur $t o$ phase changing collisions where the phase of the oscillation is perturbed, but. the quant un numbers are unchanged. In thr intrared, where energy differences are comparable to kT, 1 hr importance of phasn changing collisions is unctear. In the $1 \times 10$ code the rotatiomal rolaxatjou rate was set proporlional to the masurnd linewidth in Hl: Cood agrorment with experinent was obtained when il was assumed that at lrast one half of all 1 ine broideninf I ransilions involved a chanp; of rotational state. 
ihe rorrelat im lectwern linewidth aml rotalional

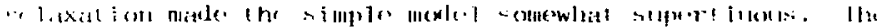

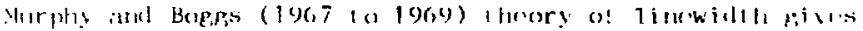

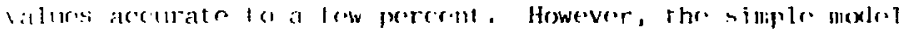
sivers insight into the physical processes. In other models

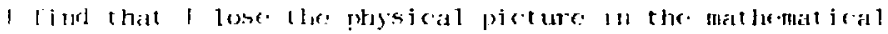
detait

A classical model will the lised alli then $r$ ortelale el with the quantized rotational levels, similar to LandauTeller theory lor vibrationel relaxation (Landau \& Teller, 1936; Herslold \& Litovitz, 1959; Rapp, 1900). The classical lheory will be shown to give rrsults similar to a quantum theors.

The model assumes two hard sphorfs with inibedrad ideal dipoles. When they are separated by a distance $R$, [moleralde 1 rxerts a torques

$$
\tau_{2}=\frac{-\mu_{1} \mu_{2}}{R^{3}}\left[2 \cos \theta_{1} \sin \theta_{2}-\sin \theta_{1} \cos \theta_{2}\right]
$$

on molraculr. 2, where $\mu_{1}$ and $\mu_{2}$ arf the dipols: moments and the angies ate shown in fig. Ul. For simplirity we will srit $\partial_{1}=J$ and let $\theta_{2}=\left(\omega_{0} t+\delta\right)$, whore $t=0$ at the instant $\sigma^{\prime}$ closest approach. The angle $\delta$, therefore, is the angle letwren the dipoles at the instant of closest approach. Thr. simplest model for translational motion, a straight line 1.rajestory itt constant velority will be used. The distance lunl wern dipoles at any time: is riven by $R^{2}=r^{2} t^{2}+b^{2}$. 
The dipoles exert torques on each other, leading to a change in rotational energy

$$
\Delta E=\int \mathrm{rd} \theta \text {. }
$$

With the above assumptions the rotational energy change in molecule 2 is

$$
\Delta E=-2 u_{1} \mu_{2} \int_{-\infty}^{\infty} \frac{\sin (\omega t+\delta)}{\left(v^{2} t^{2}+b^{2}\right)^{3 / 2}} \omega d c .
$$

This is the net energy change in a collision. The energy at some instant $t_{1}$ is obtained by letting $t_{1}$ be the upper limit in the integral. This instantaneous value of the rotational energy change is a rapidly oscillating function, and has a large magnitude when $t_{1}$ is small. As the two molecules approach, the instaneous energy change becomes large, but as they separate most of this change is cancelled by the symmetry of the trajectory. It is also interesting to note that the collision is nonadiabatic even for molecules with thermal velocities. In vibrational celaxation only rast molecules contribute. Slow molecules have adiabatic collisions where the molecules carı readjust easily during the time of encounter. In the case of rotational relaxation, even a slowly moving molecule has a nonadiabatic collision in the sense that the rotational energy change near the instant of closest approach can be very large, equivalent to a change of several quantum numbers. There is, therefore, an appreciable probability of its being in some other quantum state. 
This occurs because the dipole inceraction energy is larger than the rotational energy levels.

Before integrating Eq. D-2, we expand $\sin (\omega t+\delta)$ to $\cos \delta \sin \omega t+\sin \delta \cos \omega t$. The first term vanishes on integration because it $i:$ odd. After re-arranging the integrand we get

$$
\Delta E=\frac{-2 u_{1} \mu_{2} \omega \sin \delta}{y^{3}} \int_{-\infty}^{\infty} \frac{\cos \omega t d t}{\left(t^{2}+b^{2} / v^{2}\right)^{3 / 2}}
$$

The integration gives a modified Bessel functicn of the second kind (Abramowitz \& Stegun, 1964).

$$
\Delta E=-\left(\frac{4 \mu_{1} \mu_{2}}{b^{3}}\right)\left(\frac{\omega^{2} b^{2}}{v^{2}}\right) \text { sin } \delta K_{1}\left(\frac{\omega b}{v}\right)
$$

The first cerm is the electrostatic enezgy of the dipole-dipole interaction at a separation equal to the impace parameter, b. The ratio $\frac{a b}{y}$ is related to the ratio of the rotarional energy to the kinetic energy. The rotational energy of an $\mathrm{HF}$ molecule is approximately $E_{\text {rot }} \approx 1 / 2 \mathrm{~m}_{\mathrm{H}} \omega^{2} \mathrm{r}_{\mathrm{e}}{ }^{2}$, where $m_{H}$ is the mass of the hydrogen atom and $r_{e}$ is the equilibrium separation. Similarly, $1 / 2 \mathrm{Mv}^{2}$ is the kiretic energy. Both the energies are proportional to $k 1$, and $b>2 r_{e}$. Therefore, the quantity $\omega^{2} b^{2} / v^{2}$ is proportional to the ratio of the rotational to the kinetic energies multiplied by the ratio $\mathrm{M} / \mathrm{m}_{H}$, which is about 20 in the case of $H F$. The quantity $\omega b / v$ will range from about ? to 40 as $J$ goes from 1 to 15 if $v$ is the average velocity. The asymptotic form of $k_{1}(x)$ is $\sqrt{\pi / 2 x} e^{-x}[1+o(1 / x)]$. Using the asymptotic form gives 


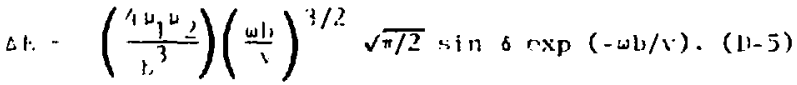

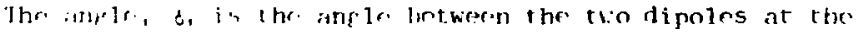
ilistant of c]osiss approact. It is necessary to averagen orer c. b, and $v$.

The average ovr's is the trickjest. At $t=-\infty$ the phase anglus are ranion, but the dipole forces tend in aljan the lipoles so that cortain values of 6 are preferred wor others. This implic.s that the instantaneuss valur a w ehanfers whon the dipoles approach each other. It. is dilijait to calculato the anragevalue of sin accurately bui it is ratijly rst imalad. Broaus of the dipole forcesi the moleculd wil? rotale slower than average over half a cycle, and aster

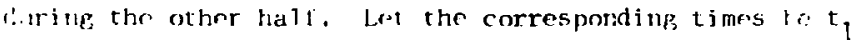
and $t_{2}$, 1 hen $t_{1}+t_{2}=2 \pi / \omega_{0}$. where $\omega_{r}$ is the averige rotational frequiney. The averape over o wit] br approximatelv $\langle$ sin $\delta\rangle-\left(1_{1}-1_{2}\right) /\left(v_{1}+t_{2}\right)$ with $?_{2}-\pi /\left(w_{0}-3 n\right)$ and $r_{2}=\pi /\left(\omega_{0}+\Delta \omega\right)$, so that $\left\langle\right.$ sill $\delta>$ is approximat $+1 y \Delta \omega / \omega_{0} \cdot$

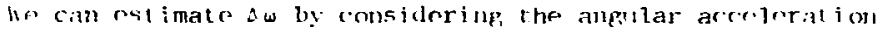
lur ia dipule-dipole forces

$$
\frac{d}{d t}=\tau / 1=\frac{2 u_{1}{ }^{\mu} 2}{t r^{3}} \quad \text { sin } \omega t \text {. }
$$

whres 1 is the torape augl I the moment of inertia. fibril

$$
\Delta \omega=1 / 2 \int_{-\pi / 2}^{\pi / 2} \pi / 1 d 1=\frac{1^{1 / 2}}{11^{3}} \int_{-\pi / 2}^{\pi / 2} \sin \omega t \omega d x,
$$


where the $1 / 2$ reproments an apperoxinate average over the

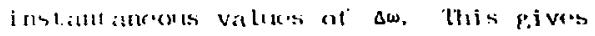

$$
\langle\operatorname{in} \delta\rangle-\underbrace{\Delta \omega}_{0}=\frac{2 \mu_{1} \omega_{1}}{b^{3} 1 \omega_{0}^{2}} \text {. }
$$

which is the ratjo of the dipolo energy at clasent approach to the rotational suregy. Our estimate has implicity atsimed $\Delta w / w_{0} \ll 1$. The uppor linit ol $|<\sin 6>|$ is 1 . For small vilues of 1 the dipole energy is large, so we can approximatr $\langle$ sin $\delta\rangle=1$. Ihis approximation is vallid except when wb is very latrger, but then the encergy change is stmall borause of tre. exponinlial term in bi D-5. he now hase.

$$
\Delta E=-\frac{4 \mu_{1} \mu_{2}}{b^{3}}\left(\frac{\omega b}{v}\right)^{3 / 2} \sqrt{\pi / 2} \operatorname{axp}(-\omega b / v) .
$$

which fllust be averaged over $b$ and $v$. The average over b cal. br. expresised as an incomplete Gamma fimetion, and taking, thr. loanitil trom of the asymptotic expansisu simply results in 1s jug " $"$, the ininimum impact parameter, for b in Eq. D-8, The average over $v$ is the samc as in the Landau-Teller tneor: cxerpt lor a different power of $v$ in front of the rxponential. The rrsult is similar with the average value of v bring, $(k T w D / N)^{1 / 3}$, with $M$ the reduced mass of the two molecules. Substituting in Eq. W-8 gives

$$
\langle\Delta \mathrm{l}\rangle=\frac{4 \mu_{1}{ }^{\mu} 2}{b_{i}^{3}} \omega b_{0} \sqrt{2^{\frac{\pi M}{k T}}} \exp \left[-\left(\omega^{2} b_{0}^{2}-M / k T\right)^{1 / 3} !(1)-9:\right.
$$

This is the classical average cnergy loss. This can be rolated to the quantum mechatical probability in a simple 
mariner (Rapp \& Kassal. 1969). A classical ensemble of $\mathbf{n}$ collisions fould have an energy loss $n\langle A E\rangle$. A correspordíng quantum ensemble woild have an energy loss $n P_{J}\left(E_{J}-E_{J-1}\right)$, where $P_{,}$is the probability of the transition from $J$ to $\mathrm{J}-1$. For rotational trinsitions, $E_{J}-E_{J-1}=2 B_{0} J$, where $B_{0}$ is the rotational constant of the molecule. Thus we have the simple relationship between the quantum probability and the classical average energy loss

$$
P_{J}=\langle\Delta E\rangle / 2 B_{0} J \text {. }
$$

Substituting this in Eq. D-9 gives

$$
P_{J}=\frac{2 \mu_{1}{ }^{\mu} 2}{b_{0}^{3} B_{0} J} \sqrt{\frac{M \omega^{2} b_{0}^{2}}{2} k T} \exp \left[-\left(\frac{M \omega^{2} b_{0}^{2}}{k T}\right)^{1 / 3}\right]
$$

The first term is the ratio of the maximum dipole interaction energy to the change in rotational energy. For highly polar molecules this can be considerably larger than one. The other terms contain an expression which is dimensionally equivalent to a rotational energy divided by the average thermal energy. However, this expression doesn't represent an actual rotation. The frequency is the initial rotational frequency of one molecule, the distance is the minimum impact parameter, and the mass is the reduced mass of the colliding molecules. The expression is proportional to the initial rotational energy. Assuming that most of the rotational energy of $\mathrm{HCl}$ is the motion cf the light-hydrogen atom about the heavy chlorine, the initial rotational energy is $1 / 2 m_{H}{ }^{2} r_{e}{ }^{2}$, where $m_{H}$ is a proton mass and $r_{e}$ is the equilibrium separation. Thus 


$$
M w^{2} b_{o}^{2}=\left(\frac{M}{m_{H}} \frac{b_{0}^{2}}{r_{e}^{2}}\right) m_{H} w^{2} r_{e}^{2} \text {. }
$$

The mass ratio, in the case of tcl is 18 and $b_{0} 3$ to $4 r_{e}$, so the term in parenthesis is about 200. The greatest uncertainty is in the value of $b_{a}$, and exceeds the error made by neglecting the motion of the chlorine atom about the center of mass. Quantum mechanically the initial rotational energy is approximately equal to $1 / 2 \mathrm{~m}_{\mathrm{H}} \omega^{2} \mathrm{r}_{\mathrm{e}}{ }^{2}$. Thus $M w^{2} b_{o}^{2} \sim 400 B_{0} J(J+1)$. For HCl $B_{0}$ is about $10 \mathrm{~cm}^{-1}$ and at $300 \mathrm{~K} \mathrm{kT} \sim 200 \mathrm{~cm}^{-1}$, so

$$
M \omega^{2} b_{0}^{2} / k T \approx 20 J(J+1) \text {. }
$$

The dipole moment of HCl is approximately 1 Debye and if $b_{0}$ is $3.5 \AA$, the dipole interaction energy is $137 \mathrm{~cm}^{-1}$.

Calculated probabilities using these numbers are shown in Table D1 and Fig. D2, where they are compared with Polanyi's fit to the experimental data,

$$
P=1.42 \exp (-.0085 \Delta E) \text {. }
$$

Clearly Eq. D-11 gives a probability which is much too low at high $\mathrm{J}$. The main problem is in the exponent. Numerical evaluation of the integrals doesn't remove the discrepancy. The error appears to be due to assuming that the target molecule is not rotating at any time. In reality, it will experience torques and begin to rotate. The torques will then oscillate at the difference frequency and reduce the exponent. To calculate this frequency would require more sophisticated mathematical techniques. It is also clear 
that a guantum treatment would not solve the problem. Firstorder perturbation theory gives the probability as the lourier transform of the interaction potential. Ihis gives roughly the same integrals as the classical theory. Dillon and Stepherison (1973) have shown that the straight path assumption can cause calculated probabjlities to be too sinal], and that the error increases with increasing rotational inergy.

This simple theory secms to give the appropriate Illapnitude for the rotational relaxation at $J=2$. The cros:, ifction decreases with increasing $J$ in agreement with -xperiment, but the decrease is much too rapid. The error appears to be due to the approximation of assuming a straight path and that the target molecule remains stationary. Some numerical calculations with the same physical model show that the target molecule does in fact rotate. The physical picture - rotational relaxation due to dipole-dipole interaction - is probably correct, because the same picture has been used successfully to calculate line broadening in several molecules (Murphy \& Boggs, 1967-79; Meredith, 1972; l'sau \& Curnute, 1962). Apparently the failuro of the moxle] in due to makhematical difficulties. 


$\begin{array}{ccc}1 & \text { Equ. 1)-11 } & \frac{1.42}{\mathrm{ex}(1+.0085 \Delta \mathrm{r})} \\ 1 & 7.1 & 1.19 \\ 2 & 1.32 & 1.01 \\ 3 & .36 & .85 \\ 4 & .11 & .72 \\ 5 & .037 & .61 \\ 7 & .0038 & .43 \\ 10 & .00037 & .26 \\ 15 & 7.2 \mathrm{E}-6 & .11 \\ 26 & 2.4 \mathrm{E}-7 & .047\end{array}$

Tihlo ;1 Comparison of calculated rotational relaxion rate (Ec. D-11) with experimental curve Fit for HC1, i.e. $1.42 \exp (-.0085 \Delta \mathrm{E})$. 


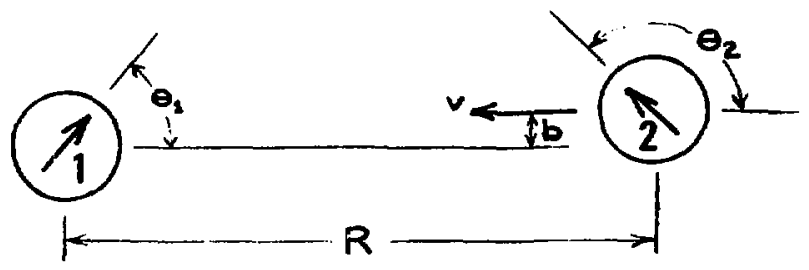

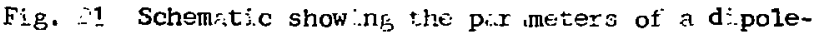
dipole inter iction model. 


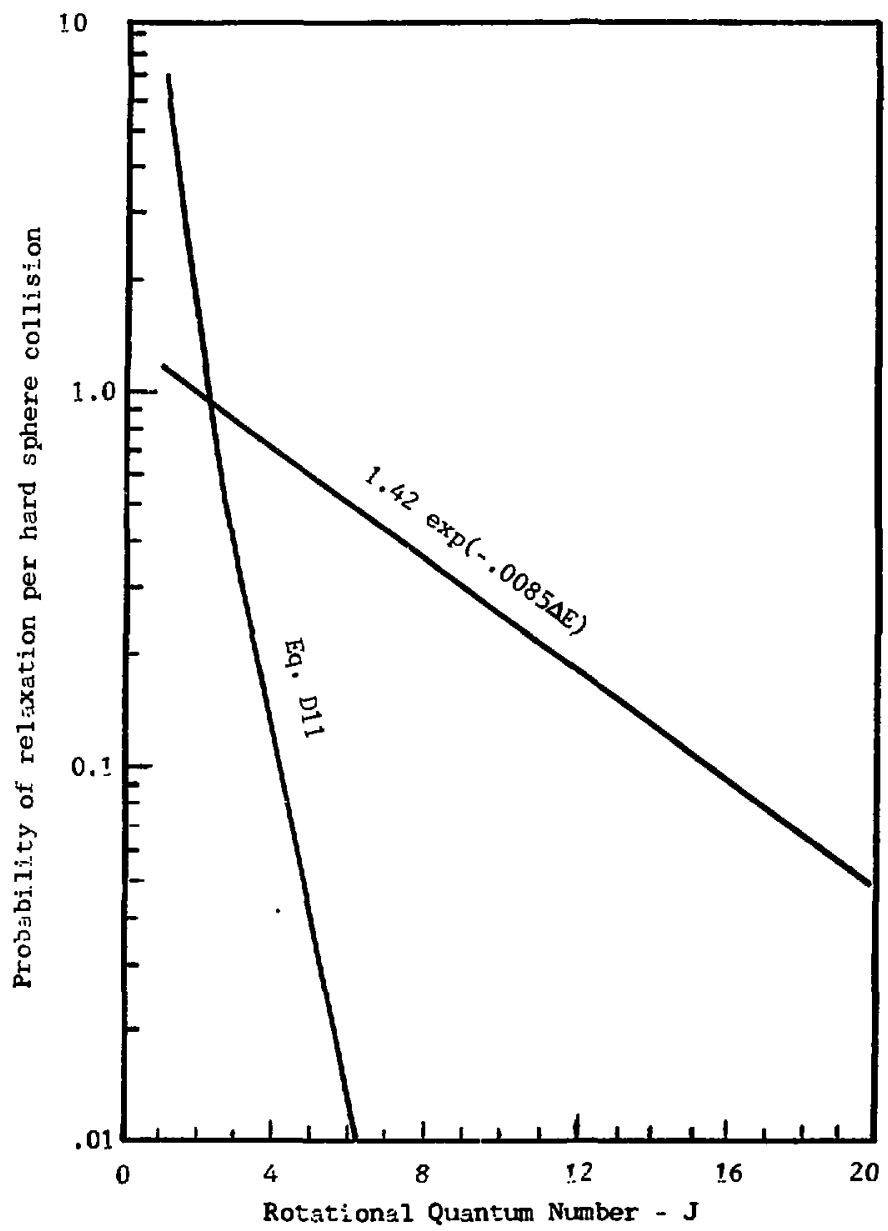

Fig. D2 Comparison of cslculated probabilizy of rotational relaxation ( $E_{1}$. 511 ) with experimentally based curve $f:=[1.42 \exp (-.0085 \Delta E)]$. 
Appendix E - Subroutines DIFFUN and THERMO from the MINOTAR Computer Code

These two subroutines form the core of the MINOTAR

code when used in conjunction with $C$. W. Gear's subroutines for integrating stiff differential equations (Gear, 1971a). The remainder of the code handles input/output and terminates the code at a preselected time.

Subroutine DIFFUN computes the rate equations for the density of each species. Some of Gear's original arguments in the subroutine call have been transferred to COMMON block PRINC.

Subroutine THERMO calculates the temperature and the thermodynamics of each species. It also calculates the forward and reverse rate coefficients at the current temperature. 
SUBRCUT INE DIFFUNICONE, OCOT I

il AUO 72

CORAENT CARDS REVISEO $*$ SEPT 1973

THIS SUGRDUT INE CALCULATES THE TIME UERIVATIVE OF THE

DENSITY OT EACH CMEMICAL SPECIES. THIS SUEROUTINE IS

INTENDED TO GE CALLED GY C.H. GEAR.S SUgROUTINE STIFF.

INPUT ARGUMENT

CONC (I) THE VALUE OF THE DENSTTY (HOLES/CCI OF CHEHICAL SPECIES I, OUTPUT ARGUTENT

DCDT(t) THE TIME DERIVATIVE OF CONC(II.

BTHER VARIAELE, AS NOTED BELOW, ARE TRANSHITTEO VIA LABELLED COHAN.

THIS SUBROUTINE CALLS SURRCUTINE THERMO HHICH RETURNS THE VALUES OF THE FOLLOLI:AS DRAAYS IY COLMLN/ THERM,

RATEFW(J) THE FORAAPO REACTIGN PATE FCA REACTION J

RATERV (J) THE CORRESPONDING KEUERSE RATE.

THE FOLLOHING ARRAYS. IN COMTHON/CHEM/. MUST HAVE BEEN SET BT THE INPUT ROUTINES

5R(I,J) THE NUMEER OF MOLECULES OF SPECIES I IN REACT:DN J ON THE REACTANT SIDE GF THE EQUATION

SPI\},J THE SAME ON THE PABOLET SIDE OF THE AEACTIOH

CAT $(1, M)$ THE NUMBER OF MOLECULES GF SPECIES I IN CATALYST $m$. VALUES OTHER THAN 1.0 OR 0.0 SPECIFY THE RELAT IVE EFFICIENCY OF SPECIES $t$, BUT DO NOT NFECT THE GALANCE.

THE FQLLOHING INTEGEAS, IN COHONIPEINC, HUST MAVE EEEN SET BT THE INPUT RDUT INES.

KMEM THE TOTAL NUMBER OF CHEHICAL SPECIES PAESENT.

KMIN = KHEM+1

IMAX = KHEM PLUS THE MUMBEA OF OEFINED CATALYSTS.

JHAX THE TOTAL NLHEER OF CHEMICAL REACTIONS FOR THE PROBLEM.

SUEROUTINE DIFFUN CALEULATES THE AUXILIARY ARAAY

RAIE

HHICH IS TRANSAITTEO TO THE OUTPUT ROUTINES VIA COMHM/CHEM,

It IS NOT OTHEHHISE USED IN OTHER SUBROUTIRES.

DEAL CONCIZO). DCOP(20)

C THE FOLLOWING LABELEO COMMON BLOCKS MUST BE CONTAINED IN THE CALLING SUGROUTINES AMO IN SUEFOUTINE THERMO.

COMYGN/PRINC/YIZO, 131 , TIME, KMEM,KMIN, IHAX, JXT, JHAX

COMHON/ THERMI OENSITY. PRESSUR, IEMP, RATEFHIIOOI, RATEAVIIOOI.

$x$ EOULKCIOOL

C

COMON/CHEM SRt20,100ג, SPt20,100), RATEt100), CATtzo,20), ISO

- - - -

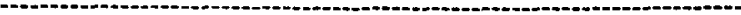

SECTION

CALCULATE THE CONCENTRATION OF THE CATALYSTS

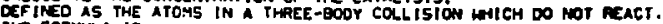

THE FORimula is

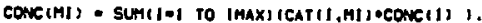

WEAE *MI. IS THE CATALYST SPECIES JNDEX

and hanges fRom Krin to IMaX.

00 I27 MI KHIN, IMAX

conte (t) 1 - 0.0

Do les 1. I. Imax

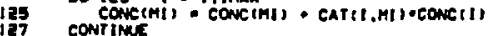

c 


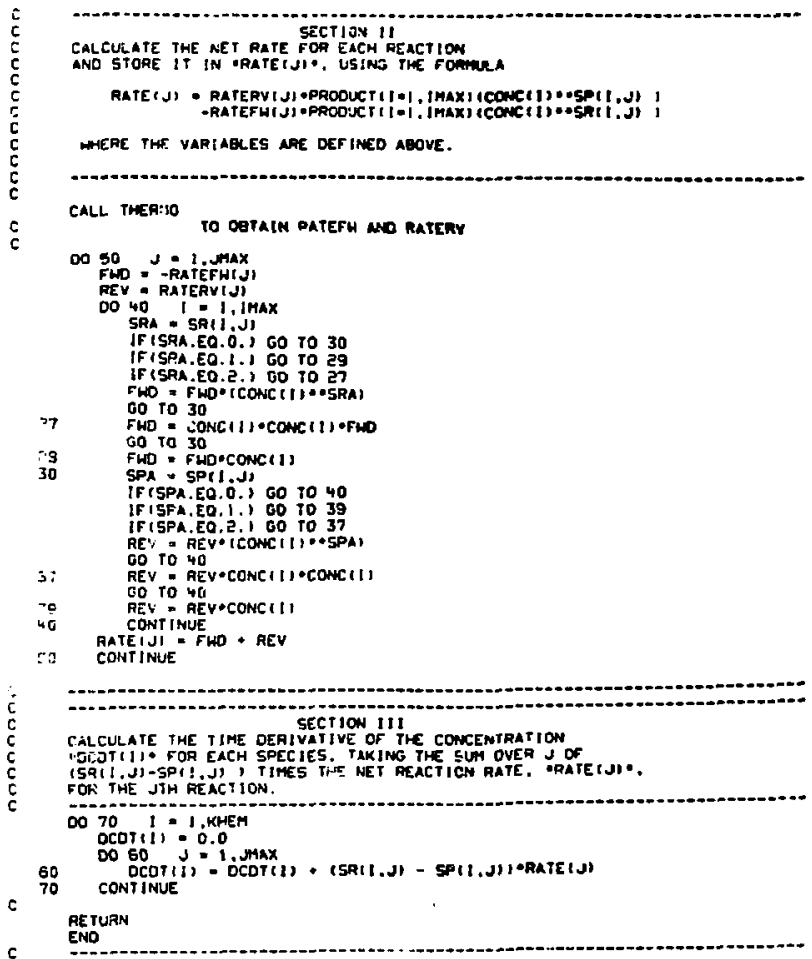




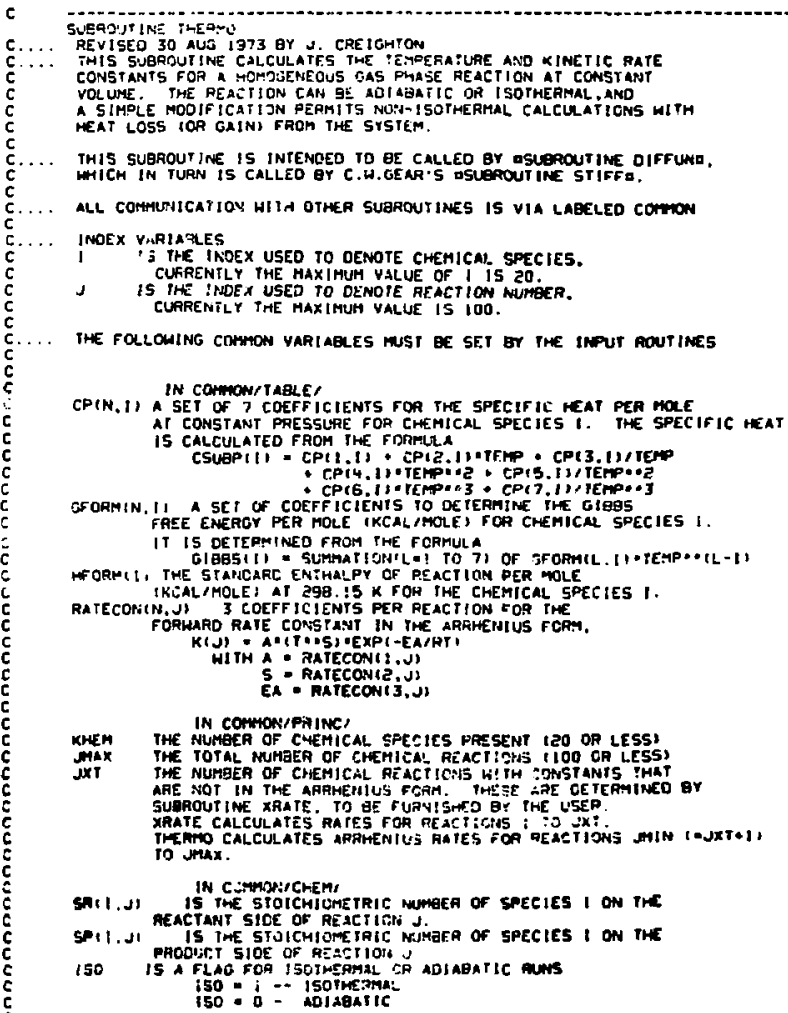

IN conmowrPininc,

KHEM THE NLMEEA OF CWEHICAL SPECIES PAESENT TEO OR LESS:

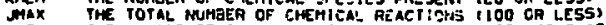

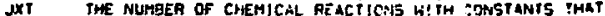

AFE NOT IN THE AARHEHIUS FCAH. IHE SE SAE OETEAMINEO BY

SUDROUTINE XRATE. TO GE FURY! GMEO B: THE USED.

XRATE CALCULATES RAIES FOR PEAC TICHS: :0 JX:

THERTO CALCULATES ARAHENIUS RAIES FOR REAETIONJ MIN ro $\operatorname{Mrax}$.

IN CDWMOAICHEM

SHII.J) IS THE STOICHIUHEIRIC MUMEE OF SPECIES I ON TH: REACTANT SIDE OF REACTIORO J.

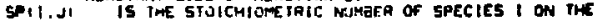

I50 PAOOj:T SIOE OF GE:ETIOH

150 is a FLAC FOA I5OTMERMAL CP ADIABATIC Aurs

iso - i ISOHERMa:

iso - o - ADIAgaile 


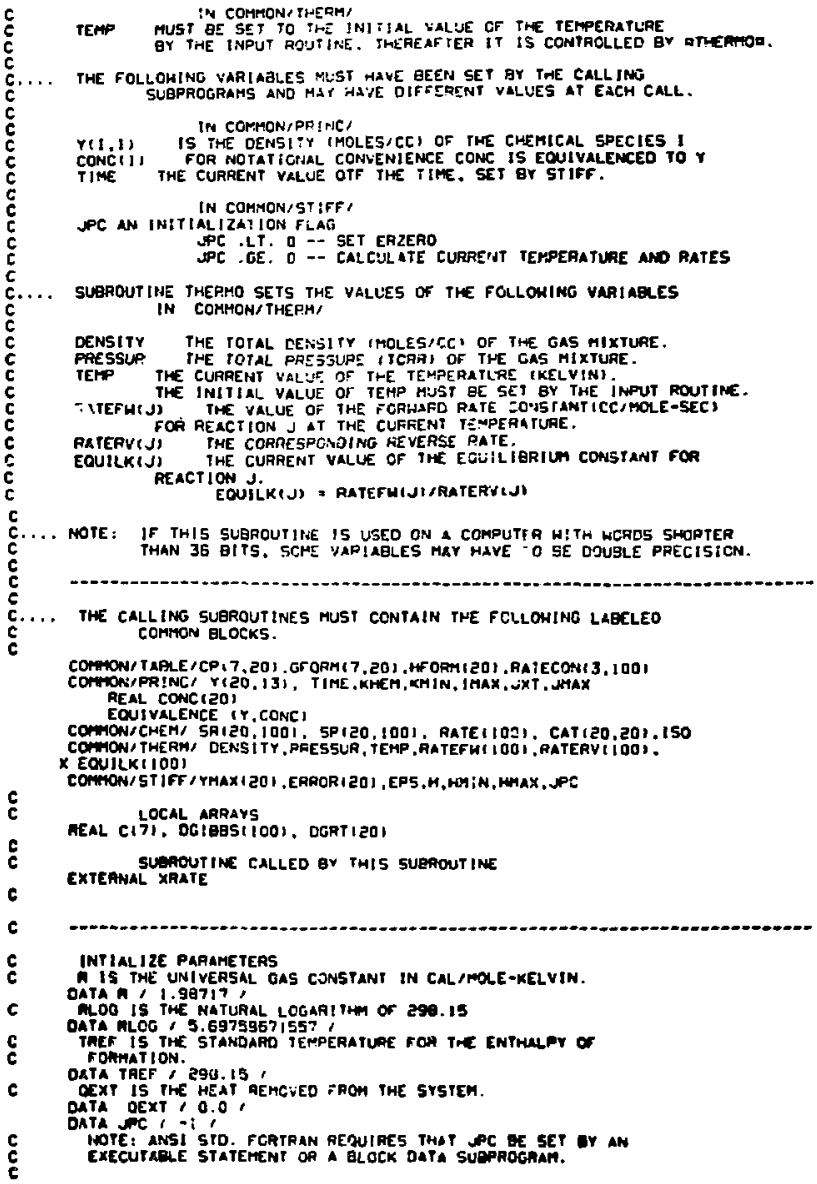




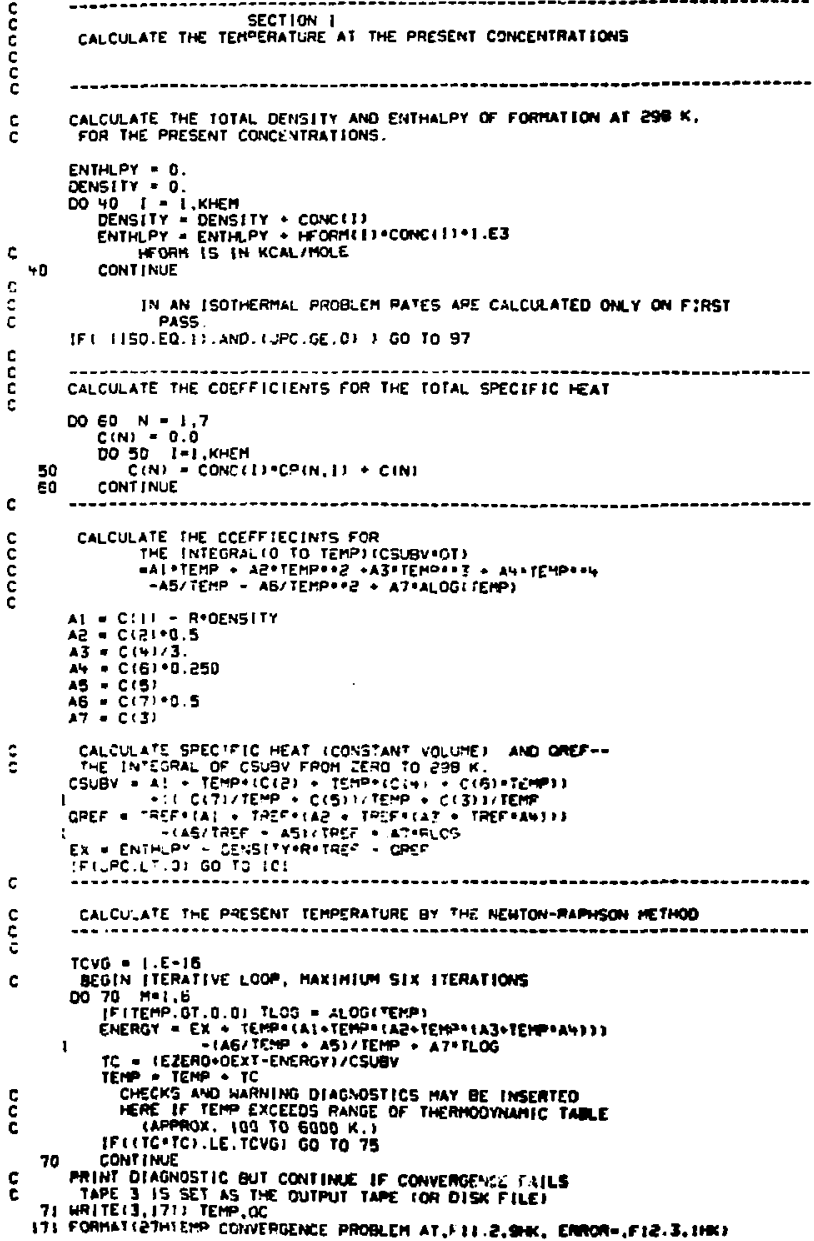




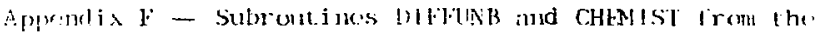
ixion cexple

Thess twe subromt ine form the core of the IXION cotr. Subrout ine MlltuNB is called by the cear subrout ine, SIIFEB, co evaluale the time derjvatives. (STIFl.B is a rersion of the Gear routiue which takes advantage of the banded nalire: o! the large Jacobian natrix (Hindmarsh, 2973). D)FFINB evaluates the electric lis pold, polarization and populat i on eytutions. It calls subroutine CHFist to get filany of the required co-effirients. CHEMIST catculates the rate of tormation of $\mathrm{HF}$ assumiles that the only important rencition is

$$
+\mathrm{H}_{2}+\mathrm{HF}+\mathrm{H} \text {. }
$$

11. also simulates the dissociation of $\mathrm{Nr}_{3}$ to give $\mathrm{F}$, and calculatros co-efficients for the vibrational relaxation. rotational rolaxation and line broadening terms. It assumes that the thermo-dynamic temperacure remains constant. 


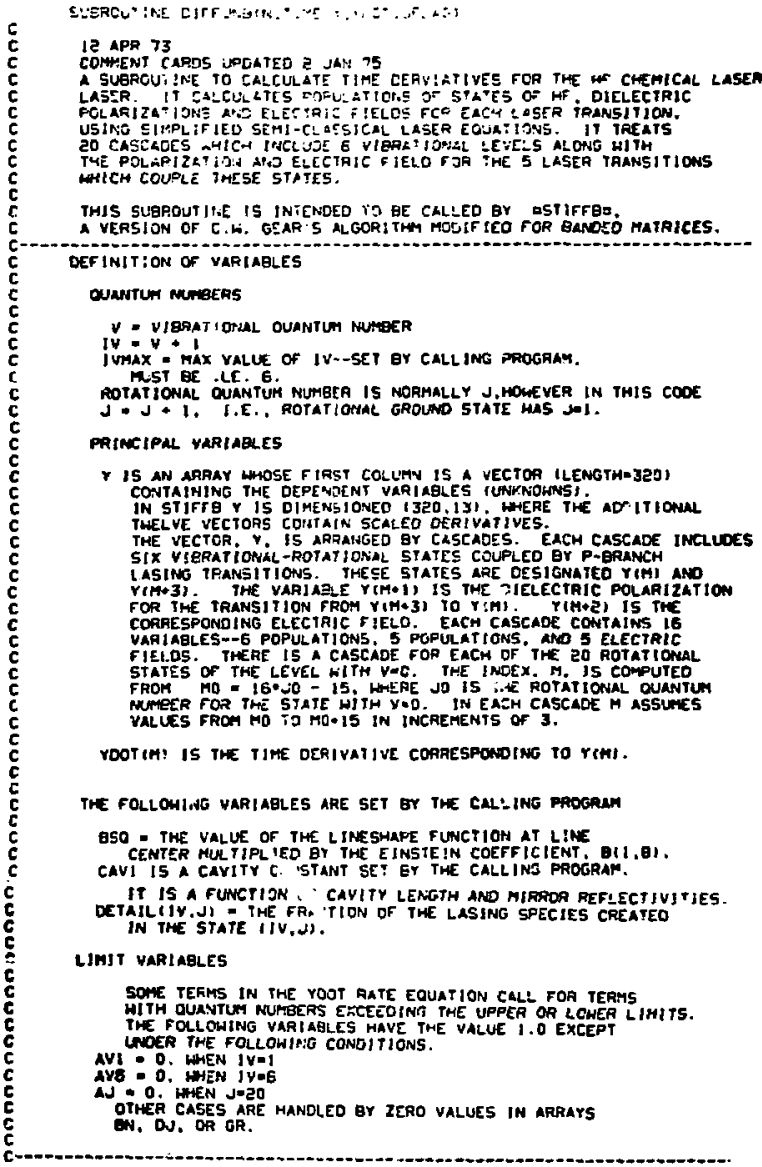


$\stackrel{c}{c}$

SET UP ARTAYS CF CONSTANTS

DIHENSION VIJEJ: VDOT(3EJ)

OIMENSION ENRE:T: DH31) GR!5t)

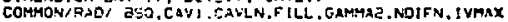

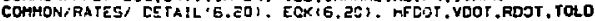

c

COHON/EQL' IERHIG,20I. TEMP.RI,RVIG, PPOT, MZ,FO

C R IS THE GAS CONSTANT IN UNITS I/CM-K

OATA R : 0.69503i,

C

GRIJ) IS THE DEGENERACY RAT10--(2j-11/(2J+1).

DATA GR, O.0. .333333. ECJOCO. .714285, .777777.

1 .818181, .646153. .66666a. .68335e, 694736.

2.904761 . 913043 . .920000. -925325, .931034,

$3.935493, .939393, .942657, .945945, .948717, .951219 ;$

$\mathbf{C}$
$\mathbf{C}$

DJIJI IS THE VARIATION OF RDTATIOYAL RELAXATION AATE AND

COLL ISIONAL BROADENING RATE HITH PUTATIONAL GJANTUH NuYGER, J.

OATA DJ, 0.0, C.EZ5, 0.5i5, 0.-30, C.34C.

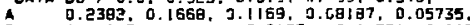

8 $0.04017,0.02814,0.0 ! 371,0.01381,0.009673$.

C $0.006776,0.004746,0.003325,0.002329,0.001632,0.0 /$

$c$
c
C

ENIJI GIVES THE YARIATION OF THE EINSTEIN COEFFICIENT HITH J; I.E. BSO・IV・g(J) IS THE PRCDUCT OF THE L INESHAPE FUNCTION AND THE EINSTEIN COEFFICIENT GOA THE PIIV.JJ TRANSITION.

DATA BN, O.0.1.5325, 1.0783, 1.0236. 1.0257.

$1.0501,1.0833,1.1225 .1 .1657,1.2121$.

2 1.2405. 1.3127, 1.3662.1.4224, 1.4762,

c

$1.5384,1.5996,1.6626,1.7274,1.7944,0.0$,

CALL CHEMIST

TO OBTAIN THE FOLLOHING VARIAOLES

- THE TOTAL RATE OF FORMATION OF THE LASING SPECIES.

VOOT IHE VT IOR VVI RELAXATION KINETIC RATE TIMES

THE OENSITY OF THE RELAXING SPECIES.

ROOT = THE RT EELAXATICL KINEIIC RATE JIMES THE OENSITY OF THE RELAXING SPECIES.

GAMMAZ = THE COLLISIONAL LINEHIOTH CONSTANT

CALCULATE DOPFLER COEF ICIENT

OPK - 2400. 'SORT (TEMP')

DIACNOST IC PRintoUT

ITTY 59

[F INOIFN,LT, 1) WRITE I TTYY, 3) POOT, VOOT, HFUOT

c

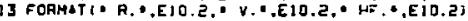




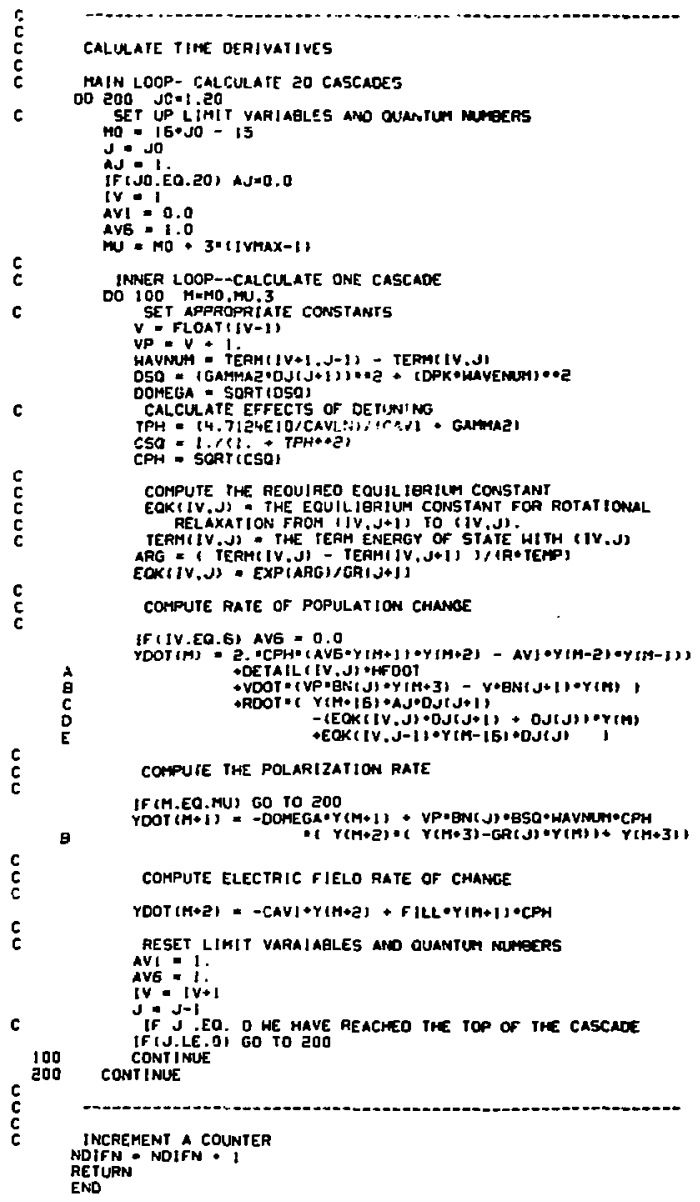




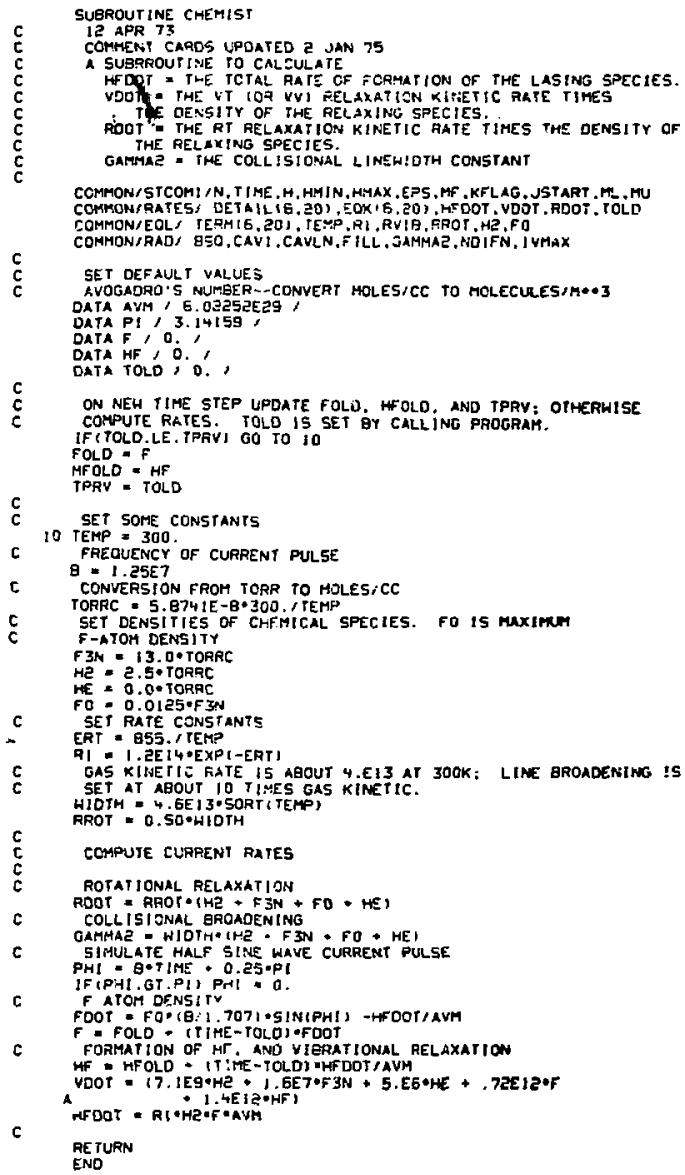

